is $x^{3}$

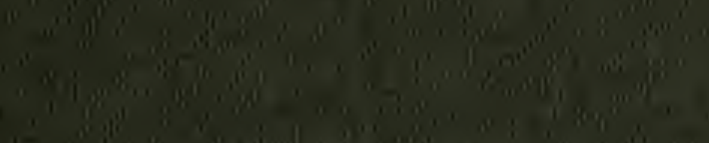

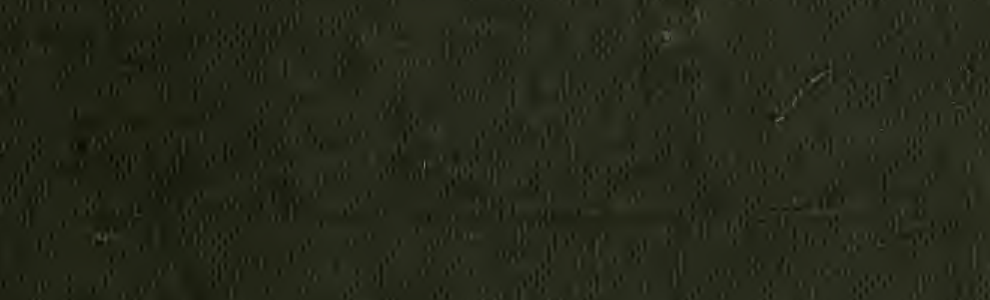

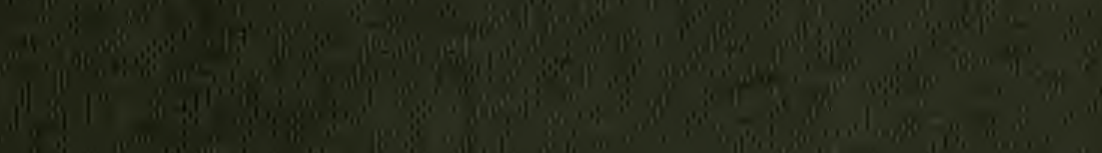
(10) (2) 


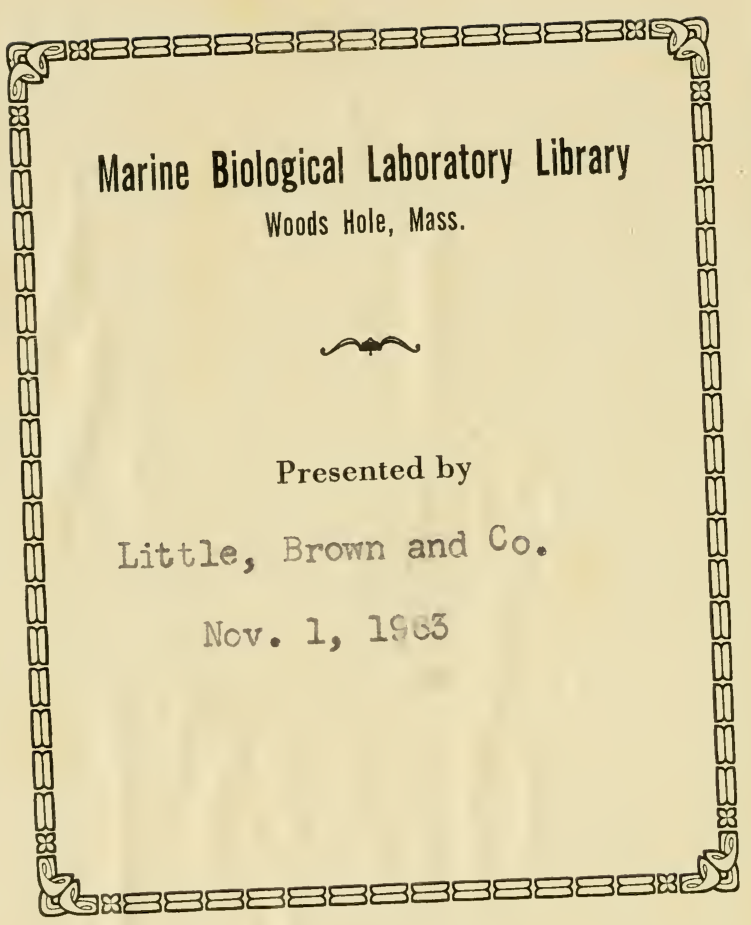




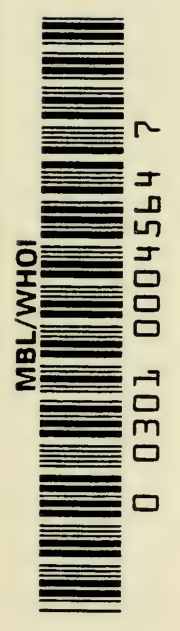




The Nature of the Natural Sciences 

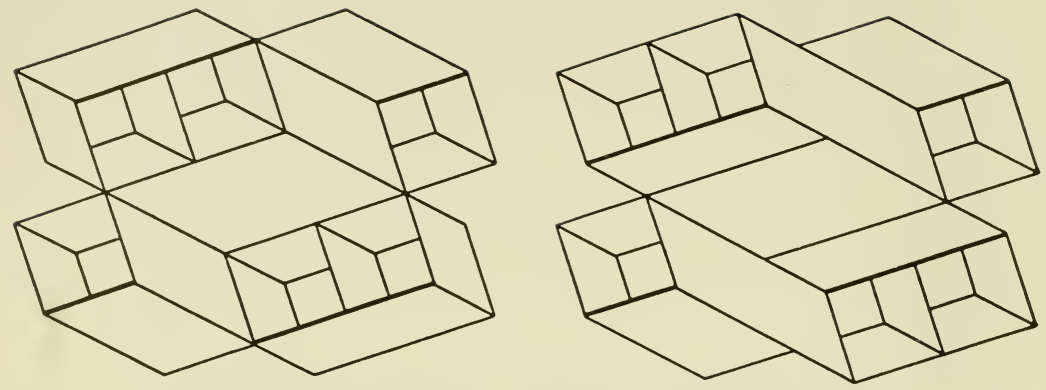

Josef Albers, A Pair of Structural Constellations:

A Matter of Changing Viewpoints

We plunge forward into the field of fresh experience with the beliefs our ancestors and we have made already; these determine what we notice; what we notice determines what we do; what we do again determines what we experience; so from one thing to another, although the stubborn fact remains that there is a sensible flux, what is true of it seems from first to last to be largely a matter of our own creation.

WILLIAM JAMES

I still believe in the possibility of producing a model of reality-that is to say, a theory which will represent things themselves.

ALBERT EINSTEIN

And the pursuit whose quest is Nature's understanding, has this among its rewards, that as it progresses its truth is testable. Truth is a "value." The quest itself therefore is in a measure its own satisfaction. We receive the lesson that our advance to knowledge is of asymptotic type, even as continually approaching so continually without arrival. The satisfaction shall therefore be eternal. 


\section{The Nature of the}

\section{NATURAL SGIENGES}

\section{LEONARD K. NASH}

Harvard University

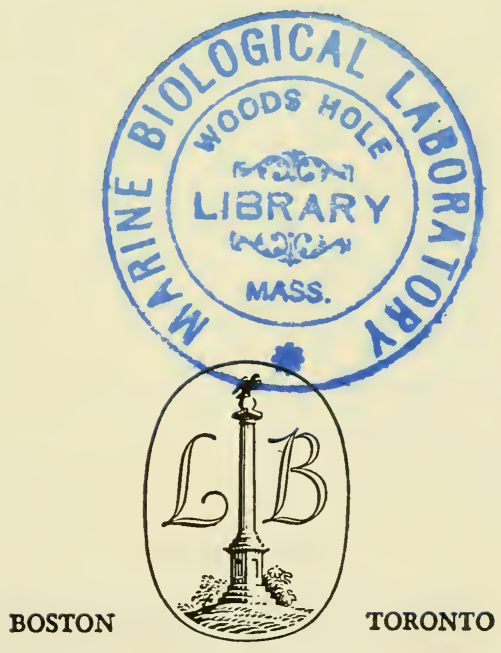

Little, Brown and Company 
COPYRIGHT (C) 1963, BY LITTLE, BROWN AND COMPANY (INC.)

ALL RIGHTS RESERVED. NO PART OF THIS BOOK MAY BE REPRODUCED IN ANY FORA WITHOUT PERMISSION IN WRITING FROM THE PUBLISHER.

LIBRARY OF CONGRESS CATALOG CARD NO. 63-17432

FIRST PRINTING

Published simultaneously in Canada

by Little, Brown \& Company (Canada) Limited

PRINTED IN THE UNITED STATES OF AMERICA 


\section{ACKNOWLEDGMENTS}

The author is grateful to the following publishers for permission to use copyrighted material. To find exact information-on the source of any specific quotation, please refer to the detailed bibliography at the end of this book.

George Allen \& Unwin, Ltd.

American Scientist

American Sociological Review

Basic Books, Inc.

G. Bell \& Sons, Ltd.

British Journal for the Philosophy of Science

Butterworth \& Co., Ltd.

Cambridge University Press

Columbia University Press

Constable and Co., Ltd.

J. M. Dent \& Sons, Ltd.

Daedalus

Diogenes

Doubleday \& Company, Inc.

Etc., A Review of General Semantics

Farrar, Straus \& Company, Inc.

Free Press of Glencoe

Harper \& Row, Publishers, Incorporated

Harvard University Press

William Heinemann, Ltd.

Holt, Rinehart and Winston, Inc.

Horizon Press, Inc.

Hutchinson Publishing Group
Library of Living Philosophers Longmans, Green \& Co., Limited Lund, Humphries \& Co., Ltd. Macmillan \& Co., Ltd. (London) Macmillan Company (New York) McGraw-Hill Book Company, Inc.

David McKay Company, Inc.

W. W. Norton \& Company, Inc.

Open Court Publishing Company

Oxford University Press

Pergamon Press

Philosophical Library

Philosophical Quarterly

Sir Isaac Pitman \& Sons, Ltd.

Princeton University Press

Routledge \& Kegan Paul, Ltd.

W. B. Saunders Company

Scientific Monthly

Simon \& Schuster, Inc.

University of California Press

University of Chicago Press

University of Pennsylvania Press

John Wiley \& Sons, Inc.

Yale University Press 



\section{Preface}

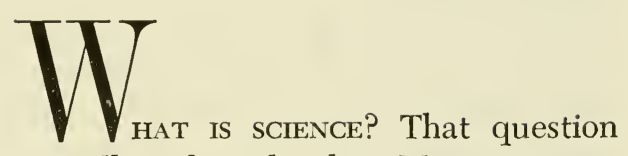

seldom emerges when scientists talk with each other. Most scientists believe they know quite well what science is and, since they all share much the same fundamental presuppositions, their beliefs rarely broach a subject for debate. However, the challenging experience of teaching students who do not intend to become scientists -students willing to doubt fundamental presuppositions to which they feel no commitment-has led me to a critical re-examination of beliefs I once held unquestionable. In this book I have tried to explain the lines of reasoning and the kinds of considerations that have led to what seems to me now a more tenable set of beliefs. Perhaps this analysis will have some value for others (both students and teachers) who-having adopted the familiar textbook stereotype-are yet not fully satisfied with this orthodox answer to the question: "What is science?" I do not deceive myself that my answer will prove acceptable to everybody, but I do believe that the reader who joins me in exploring the paths here laid out will come at least to a better understanding of the full scope of that not-so-simple question. He will, I hope, find that I have cleared a way for him through two major difficulties that ordinarily block the road to understanding.

First: I have sought everywhere to deal with "real" science, as it has been created and appraised by "real" scientists. The "ideal" science analyzed in neat philosophic syllogisms may be attractive in its straightforwardness, but it is lamentably "ideal" in that nothing like 
it has ever existed in this world. In order to escape all temptation to treat science at this dangerously extreme level of abstraction, I have throughout made a systematic effort to develop general ideas in the context of actual events in the history of science. I say nothing of what science could or should or might be, or of what scientists could or should or might think. I have instead said only what I believe science has been and is, and what scientists have thought and do think. I believe their practice and beliefs distorted only to the extent that I have tried to make explicit views that are often only implicit, and to the extent that I have emphasized the enormous area of agreement at the expense of the small but significant areas in which scientists disagree.

Second: I have tried to give a complete and integrated representation of science, with a just apportionment of attention to science as such and to its history, philosophy, internal organization, social ties, and so forth. I have tried to dissect the total problem, so that its "parts" can be seen clearly; but I have tried to reassemble the "parts" in order that they may be seen correctly. In their natural context there are between them crucially important interactions which cannot be grasped when, as ordinarily, "parts" are treated in complete abstraction from one another. The perspective on science thus developed can treat only cursorily some topics that have been treated in depth by others but, if I have succeeded, this perspective should have a breadth and balance not to be found elsewhere-simply because the depiction of real science "in the round" is so very rarely essayed.

The reader of this work needs no understanding of higher mathematics, nor of the intricacies of quantum mechanics and relativity. Less than half a dozen algebraic equations appear in the whole of the book and, of course, some loss of coverage is thus entailed. However, I conceive that the most fundamental questions relative to the nature of science were broached long before the advent of contemporary physics, and that these questions can be treated quite adequately in simpler contexts. But even if these examples should prove obscure, the technically untrained reader will, I think, still find himself quite well able to appraise the validity of the basic arguments which-like the conclusions drawn from them-are largely independent of the technical examples I have used to document and illustrate them. 
A word about the organization of this inquiry may come appropriately at this point. The table of contents will offer the reader some general indications of the lines of argument to be pursued, and will at the same time indicate to him the relations envisaged by the author among the various sub-topics considered. Chapters I, II, and III represent a discursive survey of the whole area to be explored. Here I introduce the categories to be used subsequently in systematic analyses of the many problems that emerge from the preliminary survey. If these three opening chapters prove difficult, they need only be skimmed at a first reading: their rationale will become evident after the succeeding chapters have been read. The nature and organization of the remaining nine chapters are dictated by the examination of the anatomy of science that concludes Chapter III. The social milieu of science is the subject in Chapter IV. In Chapter V the reader will find a consideration of the various characteristics of scientific "laws," and, in Chapter VI, a discussion of the nature and use of the empirical procedures deployed by scientists. The core of the book is represented by Chapters VII, VIII, and IX, which explore in detail the principles and theories that are the hallmarks of science. In the three concluding chapters I have treated science as a social institution (in Chapter X), science as an individual achievement (in Chapter XI), and science as a genuine discovery of the "real world" (in Chapter XII).

I should, perhaps, comment on the abundant use that has been made of direct quotations. Sometimes they offer particularly happy expressions of the points to be made; sometimes they serve to indicate the sources of arguments not otherwise attributed; sometimes they figure as citations of authority in areas of discourse where I myself can claim no authority; occasionally they may be no more than landmarks in my own education-statements that first resolved problems that had bothered me. Above all, these quotations are used to display faithfully the opinions of men who have themselves made scientific history. I acknowledge with thanks the courtesy of the many publishers, listed on $\mathrm{p} . \mathrm{v}$, who have permitted me to quote from works appearing under their imprint.

To deal adequately with all parts of the subject I have set myself, one would have to be deeply versed in history, psychology, sociology, and philosophy-not to mention all the individual sciences. Given a keen awareness of my own limitations, I am particularly grateful for the counsel I have received from friends and colleagues. I owe to 
J. B. Conant, whom I was once fortunate enough to serve as teaching assistant, my first introduction to some of the problems here discussed, as well as many pointers along the lines here developed. With T. S. Kuhn I long enjoyed an active teaching collaboration and, though we seem to have arrived at rather different conclusions, many of my themes were first suggested by hard-fought discussions with him. The entire manuscript has been read by Joel Cohen, Robert S. Cohen, Wendy Doniger, Gerald Holton, Thomas.S. Kuhn, Ernest Nagel, Peter Urnes, and Eleanor Webster-to each of whom I am indebted for an extended commentary that has furnished a wealth of valuable suggestions. In matters of detail I have had many helpful criticisms from K. D. Clouser, Susan and Stanley Goldberg, James Haber, F. L. Holmes, Stephanie Pfaff, and Michael Simon. My wife has devoted long hours to helping me with the reading of proofs. The skill and patience of Ronald Q. Lewton, who edited this book, have been a strong support in times of need. The unstinted assistance of the foregoing persons has saved me from many serious errors; any that remain are the indivisible responsibility of the author.

Leonard K. Nash 


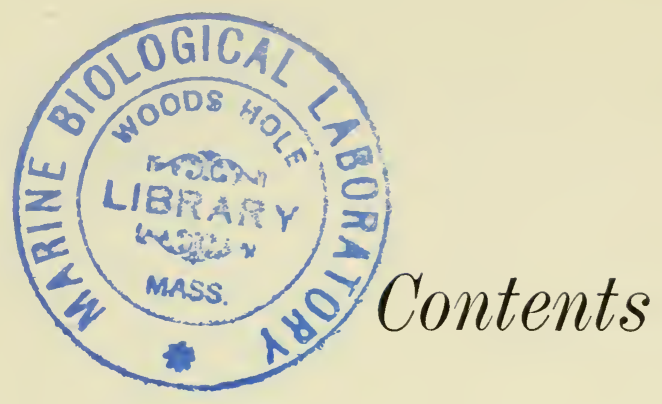

Chapter I

COMMON SENSE (AND SCIENCE)

Common sense and prevision $\ldots \ldots \ldots \ldots \ldots \ldots \ldots$

The Organization of Experience ................. 6

FROM STIMULI TO CONSTRUCTS $\ldots \ldots \ldots \ldots \ldots \ldots \ldots \ldots \ldots$

Observing $\ldots \ldots \ldots \ldots \ldots \ldots \ldots \ldots \ldots \ldots \ldots \ldots \ldots \ldots \ldots \ldots$

Constructs ......................... 10

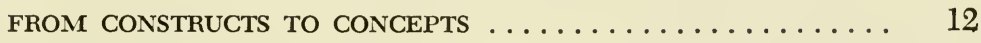

The normative factors $\ldots \ldots \ldots \ldots \ldots \ldots \ldots \ldots \ldots$

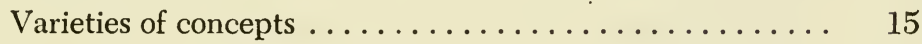

The tool function of concepts . . . . . . . . . . 17

FROM CONCEPTS TO COLLIGATIVE RELATIONS .......... 18

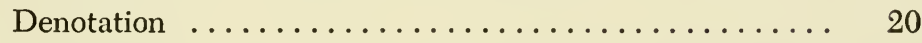

A FOURTH STAGE IN THE ORGANIZATION OF EXPERIENCE ..... 22

Similarities in intent, presuppositions, and subject matter . . 25

Some differences: Progressivism and impressiveness .... 27

Chapter \】

SCIENCE (AND COMMON SENSE)

SOME "METAPHYSICAL PRINCIPLES" OF SCIENCE $\ldots \ldots \ldots \ldots$ 
xii CONTENTS

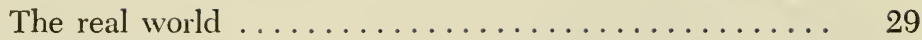

Determinism ......................... 30

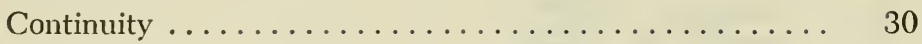

Dissolubility ........................... 31

The Subject Matter of Science .................. 31

RECURRENCE AND REPRODUCIBILITY ............. 31

Facts historical and scientific ............. 33

Uniqueness and identity .................... 34

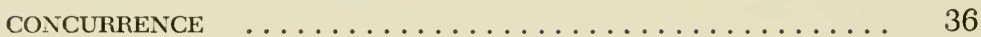

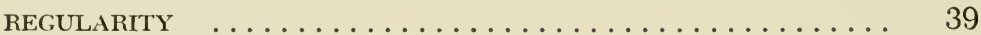

DATA AND JUDGMENT . . . . . . . . . . . . . . . 42

The Organization of Experience ................ 43

CONSTRUCTS .......................... 43

SCIENTIFIC CONCEPTS $\ldots \ldots \ldots \ldots \ldots \ldots \ldots \ldots \ldots \ldots \ldots$

The strangeness of scientific concepts .......... 46

Concepts and the anatomization of experience ....... 48

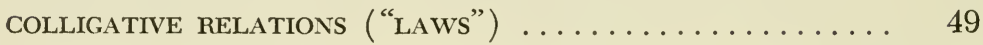

The law of the lever ................. 50

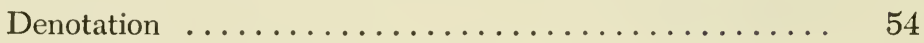

The ensemble of colligative relations .......... 55

SCIENTIFIC THEORIES ..................... 57

The kinetic theory $\ldots \ldots \ldots \ldots \ldots \ldots \ldots \ldots \ldots \ldots \ldots \ldots$

Remembering, understanding, discovering ........ 60

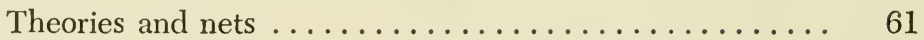

Chapter III

THE ANATOMY OF SCIENCE 63

The Evolution of Science ...................... 63

The principle of intelligibility $\ldots \ldots \ldots \ldots \ldots \ldots \ldots .65$

SOME DIFFICULTIES ..................... 67

The choice of problems $\ldots \ldots \ldots \ldots \ldots \ldots \ldots \ldots, 67$

Heuristic tools .................... 67

Organization $\ldots \ldots \ldots \ldots \ldots \ldots \ldots \ldots \ldots \ldots \ldots \ldots \ldots \ldots$ 
The social setting $\ldots \ldots \ldots \ldots \ldots \ldots \ldots \ldots \ldots \ldots$

The idea of progress $\ldots \ldots \ldots \ldots \ldots \ldots \ldots \ldots \ldots$

A FIRST QUALIFICATION: PATENCY, PROGRESS, AND PRAGMATISM ................. 72

A SECOND QUALIFICATION OF THE

PRINCIPLE OF INTELLIGIBILITY $\ldots \ldots \ldots \ldots \ldots \ldots \ldots \ldots$

Autonomous science and sciences ............. 74

A THIRD QUALIFICATION OF THE

PRINCIPLE OF INTELLIGIBILITY $\ldots \ldots \ldots \ldots \ldots \ldots \ldots \ldots$

The appeal to experience $\ldots \ldots \ldots \ldots \ldots \ldots \ldots \ldots$

A new conception of scientific method .......... 79

The principle of corrigible fallibility $\ldots \ldots \ldots \ldots \ldots .83$

The Body Scientific ......................... 84

The anticipatory apparatus $\ldots \ldots \ldots \ldots \ldots \ldots \ldots .66$

The heuristic apparatus $\ldots \ldots \ldots \ldots \ldots \ldots \ldots$

Chapter 1 V

COSMOLOGY AND TECHNOLOGY

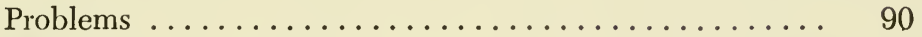

Motivation ...................... 90

Support .................... 91

Cosmology ................................ 92

Inclusiveness . . . . . . . . . . . . . . . . . . . 92

Science metamorphosed $\ldots \ldots \ldots \ldots \ldots \ldots \ldots \ldots . \ldots 2$

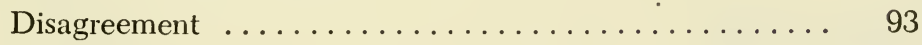

Rigidity and revolution $\ldots \ldots \ldots \ldots \ldots \ldots \ldots \ldots . \ldots \ldots$

THE Climate OF OPINION .................. 95

The linguistic factor $\ldots \ldots \ldots \ldots \ldots \ldots \ldots \ldots . \ldots \ldots$

The aesthetic factor $\ldots \ldots \ldots \ldots \ldots \ldots \ldots \ldots . \ldots 9$

The ethical factor ................... 101

The moral factor ...................... 102

THE COSMOLOGY OF ORGANIZED SCIENCE . . . . . . . . . . 104

Technology ............................. 105

Conceptual exchanges $\ldots \ldots \ldots \ldots \ldots \ldots \ldots \ldots \ldots$ 
xiv CONTENTS

Material exchanges ................... 108

SCIENCE AND TECHNOLOGY $\ldots \ldots \ldots \ldots \ldots \ldots \ldots \ldots \ldots . \ldots 109$

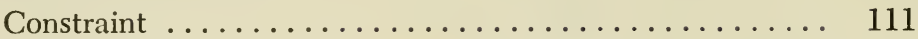

THE REPROBATION OF SCIENCE $\ldots \ldots \ldots \ldots \ldots \ldots \ldots \ldots \ldots . . \ldots \ldots$

Chapter V

COLLIGATIVE RELATIONS AND SCIENTIFIC LAWS 114

THE EFFICIENCY OF COLLIGATIVE RELATIONS . . . . . . 115

The sulfur relation $\ldots \ldots \ldots \ldots \ldots \ldots \ldots \ldots \ldots \ldots \ldots \ldots$

A COLLIGATIVE RELATION: BOTH INVENTION AND DISCOVERY . . 118

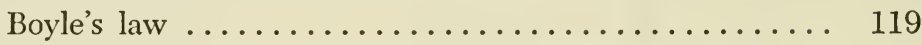

DENOTATIONS: IF INDIRECT, YET SOLIDLY ESTABLISHED . . . . . 123

Galileo's law of free fall ................ 125

A relation of Euclidean geometry .............. 127

THE “PROOF” AND ENDURANCE OF COLLIGATIVE RELATIONS . . . 127

The relation of metals ................. 129

Colligative Relations not Wholly Independent of Theories . . . 130

The Bode-Titus law . . . . . . . . . . . . . . . . . 132

CONNOTATIONS AS WELL AS DENOTATIONS ........... 133

Moseley's law ..................... 133

The Mendelian laws .................. 135

COLLIGATIVE RELATIONS IN OTHER GUISES ........... 137

Chapter VI

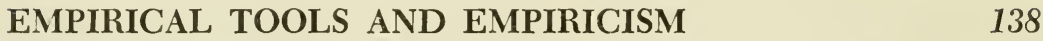

INSTRUMENTS . . . . . . . . . . . . . . . . . . . 138

The operating protocol ................ 139

Pointer readings ..................... 141

THE EXTERNAL STANDARD OF REFERENCE . . . . . . . . 143

"Things" as standards .................... 145

Techniques as standards .............. 146 
Colligative relations as standards . . . . . . . . . 147

PRIMARY AND SECONDARY DENOTATIONS ........... 147

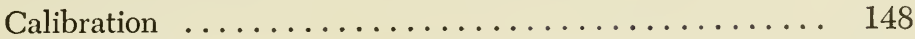

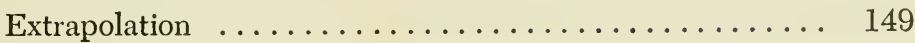

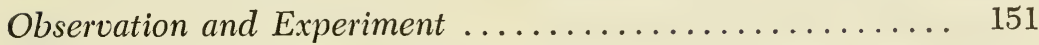

EMPIRICISM AND THE INTERNAL DYNAMICS OF SCIENCE . . . . 155

The Scientific Method ...................... 157

FINDING THE PROBLEM $\ldots \ldots \ldots \ldots \ldots \ldots \ldots \ldots \ldots \ldots$

THE DESIGN AND CONDUCT OF EXPERIMENTS .......... 161

The choice of variables ................ 162

The choice of observables ............... 163

DRAWING CONCLUSIONS FROM EXPERIMENTS .......... 165

THE POWER OF STATISTICAL METHODS ........... 166

The limitation of statistical methods $\ldots \ldots \ldots \ldots \ldots \ldots 167$

THE MYTH OF METHOD ................... 168

Chapter VII

THE PRINCIPLES OF SCIENCE 170

The Existence of a Real World .................. 172

The Principle of Intelligibility ................. 172

FOUR METHODOLOGICAL COROLLARIES ............. 172

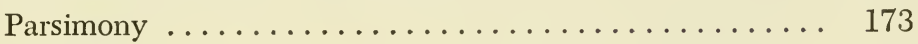

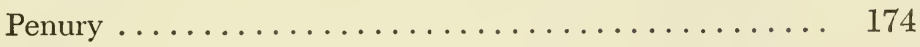

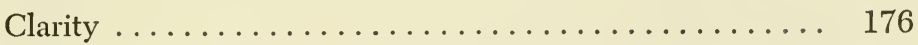

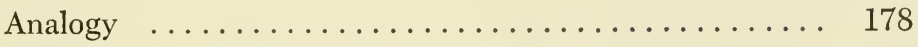

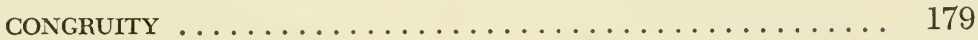

Simplicity sigillum veri $\ldots \ldots \ldots \ldots \ldots \ldots \ldots \ldots \ldots 1$

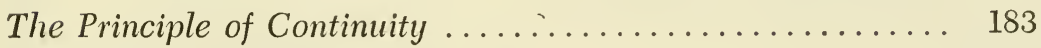

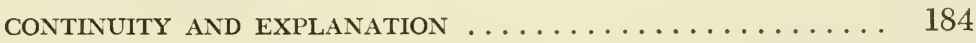

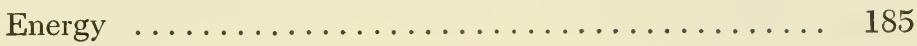

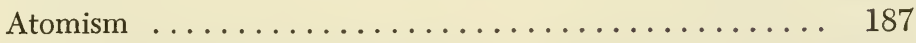

DISSOLUBILITY AND SUPERPOSITION $\ldots \ldots \ldots \ldots \ldots \ldots \ldots \ldots . \ldots \ldots$

Superposition not summation . . . . . . . . . . 190

Superposition and explanation .............. 193 
The Principle of Determinism, and Causality ............ . 194

Causality as heuristic maxim .............. 196

QUANTUM MECHANICS, DETERMINISM, AND CAUSALITY . . . . . 199

The Principle of Corrigible Fallibility . . . . . . . . . . . . . 203

LEARNLNG PRESUPPOSES ERROR . . . . . . . . . . . . . 204

THE MUTUAL CONTROL OF FACTS AND THEORIES . . . . . . 206

Substantive Principles ........................ 208

Substantive principles as conventions .......... 211

Chapter V】】

\section{THEORIES AND MODELS}

Logic and Mathematics ....................... 217

Abstract formalism and scientific law ........... 221

INDUCTION . . . . . . . . . . . . . . . . . . . . . . 222

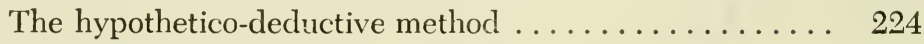

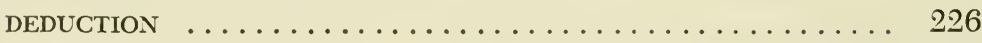

Models ............................... 230

Nodel implicit in formalism .............. 232

Formalism implicit in model ............. 233

MODELS AND SEMTANTIC RULES ................. 234

First case: Direct denotations and simple models . . . . . 234

Second case: Indirect denotations and simple models . . . . 236

Third case: Indirect denotations and hierarchic models . . 237

THE "SUPERFLUITY" OF THE PHYSICAL MODEL . . . . . . . . . 239

The positivists" "theory without superfluity" ......... 242

Heuristic power from "superfluous explanation" ....... 248

The model taken seriously ............... 250

Chapter IX

THE EVOLUTION OF SCIENTIFIC THEORIES

THE CRUCIAL EXPERIMENT $\ldots \ldots \ldots \ldots \ldots \ldots \ldots \ldots \ldots .256$ 
Situations of deadlock ................ 257

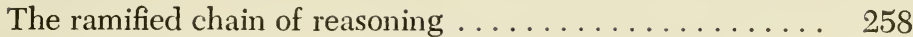

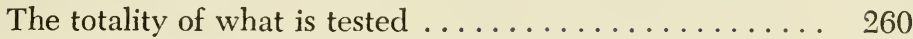

The crucial experiment in practice ............ 263

Three Functional Criteria ............................. 264

CORRELATIVE EFFICIENCY ...................... 264

The insufficiency of the correlative index . . . . . . . 266

EXPLANATORY APPEAL ..................... 267

The two criteria of immediate judgment ......... 268

HEURISTIC POWER . . . . . . . . . . . . . . . . . . . . . . 269

Natural Selection of Scientific Theories ............... 272

Viability and validity ................ 274

THE LIFE CYCLE OF A SCIENTIFIC THEORY . . . . . . . . . 276

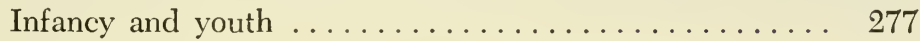

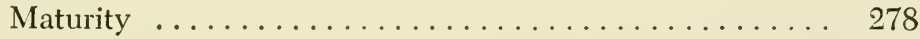

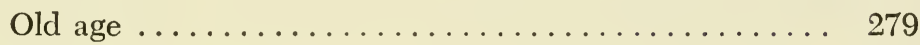

The death struggle ................. 282

Scientific Revolutions? ............................ 284

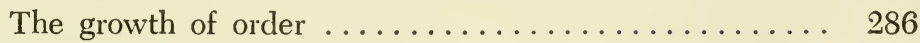

The correspondence principle ............. 287

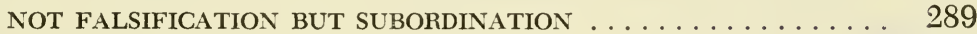

The "problem" of dual description . . . . . . . . . . . 292

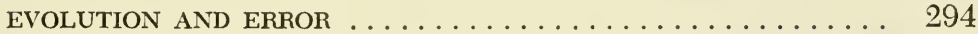

Chapter X

\section{ORGANIZED SCIENCE}

The Invisible College . . . . . . . . . . . . . . . . . . . . . . 298

COMMUNAL ADMINISTRATION, CONSENSUS, CULTURE ...... 300

THE NORMATIVE FUNCTIONS . . . . . . . . . . . . . . . . 302

Protection ..................... 303

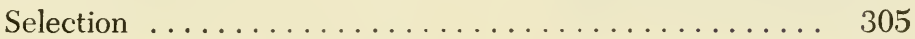

Appreciation ..................... 307

THE CO-ORDINATION OF EFFORT . . . . . . . . . . . . . . 309 
The Super-Personal Intelligence ................. 311

Complementarity of capacities ............. 312

Complementarity of skills ............... 313

Complementarity of temperaments ........... 313

Complementarity (and cancellation?) of commitments ... 314

PERTURBATIONS OF THE MECHANISM OF SELECTION ....... 315

Defective personal commitment ............ 316

Excessive authority $\ldots \ldots \ldots \ldots \ldots \ldots \ldots \ldots \ldots \ldots \ldots$

Chapter XI

CREATIVE SCIENCE 319

SCIENTIFIC METHOD: SHADOW AND SUBSTANCE . . . . . . 320

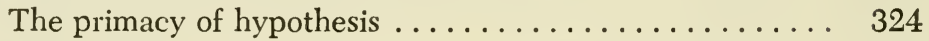

The Attitude of the Investigator . .............. 326

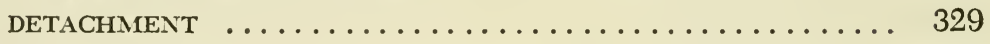

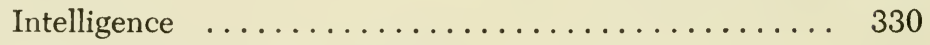

Detachment from commitment ............. 331

Accidental Discoveries .......................... 333

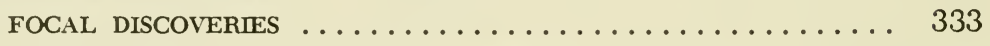

THE THRESHOLD OF IMPRESSIONABILITY ........... 335

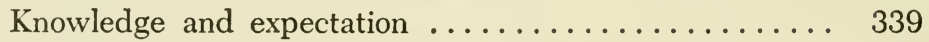

Intelligence and temperament ............ 340

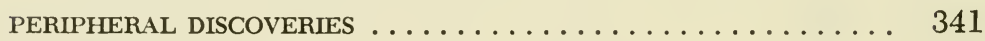

The Creative Imagination ................... 343

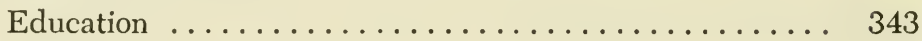

Youth ............................ 344

Two fields of view ................. 346

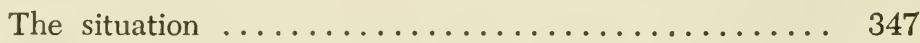

The $\operatorname{man} \ldots \ldots \ldots \ldots \ldots \ldots \ldots \ldots \ldots \ldots . \ldots \ldots . \ldots \ldots$

THE BIRTH OF INSIGHT $\ldots \ldots \ldots \ldots \ldots \ldots \ldots \ldots \ldots \ldots \ldots$ 


\section{Chapter XII}

THE REAL WORLD

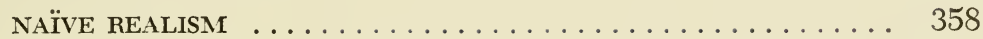

PHENOMENALISM $\ldots \ldots \ldots \ldots \ldots \ldots \ldots \ldots \ldots \ldots \ldots \ldots \ldots \ldots$

Illogical cynicism . . . . . . . . . . . . . . . . . . . . 362

REPRESENTATIONALISM $\ldots \ldots \ldots \ldots \ldots \ldots \ldots \ldots \ldots \ldots . \ldots \ldots$

Beyond Inventions-Discoveries! . . . . . . . . . . . . 365

Science and prediction ............... 366

THEORIES . . . . . . . . . . . . . . . . . . . . . . . . 368

Heuristic power .................. 369

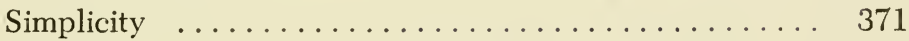

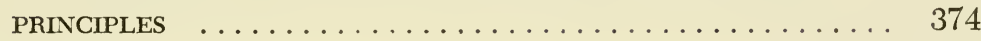

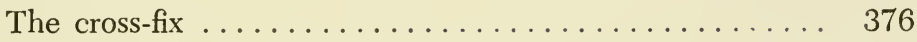

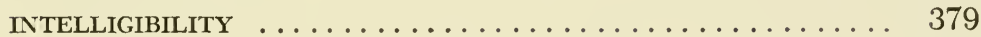

Ultimate unintelligibilities? . . . . . . . . . . 380

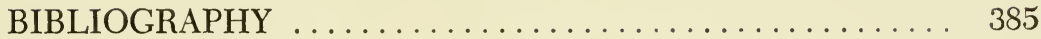

INDEX OF NAMES .................... 401 
The Nature of the Natural Sciences 



\section{CHAPTER I}

\section{Common Sense (and Science)}

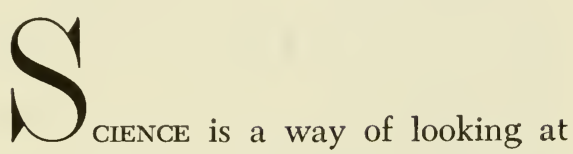

the world. There are, of course, other ways. The man of common sense sees the world in his own way. So does the artist, the philosopher, the theologian. The view of the scientist, if at all unique, is characterized by its heavy involvement of elements drawn from all the others. Like the man of common sense, the scientist views the world within the co-ordinate system provided by a framework of concepts. But, not content with the common-sense view of the world, the scientist uses a different conceptual framework. He seeks a higher unity, a deeper understanding, unknown to common sense. He devises new concepts; with them he seeks and finds novel and profound connections between apparently unrelated phenomena, as well as significant differences between phenomena apparently closely related. With the artist, then, he comes to see the world in new perspectives, with a new pattern of highlights and shadows, involving new associations, new points of emphasis-and often effective neglect of precisely those relations or connections that loom most prominently in the view of common sense. William James writes:

What we say about reality thus depends on the perspective into which we throw it. . . .

. . . By our inclusions and omissions we trace the field's extent; by our emphasis we mark its foreground and its background; by our order we read it in this direction or in that. We receive in short the block of marble, but we carve the statue ourselves. 
Novelty of viewpoint is a work of imagination. And, to be sure, imagination is valued in both artists and scientists. Proust suggests the artist charms us by teaching us to see another universe; the scientist charms us similarly, by showing us a conceptual order in the chaos of perceptual experience. But, through this same concern for rational unity, the scientist is borne on toward the viewpoint of the philosopher. "Philosophy has often been defined," says James, "as the quest or the vision of the world's unity." The theologian may conceive this unity very differently, and use very different criteria in judging the extent to which it has been achieved. But he believes as firmly as the philosopher or the scientist in the unity of the world.

The biologist W. R. Thompson, the chemist Conant, and the mathematicians Clifford and Bronowski have all written works with titles juxtaposing science and common sense. Science has, indeed, been defined (by T. H. Huxley) as "organized common sense." In the present age of relativistic and quantum physics many, including both scientists and laymen, find outrageous all comparison of science with common sense. To them science seems flatly to contradict common sense wherever it does not wholly ignore it. Yet consider the testimony of an eminent theoretical physicist of our own age: Oppenheimer urges that we

distrust all the philosophers who claim that by examining science they come to results in contradiction with common sense. Science is based on common sense; it cannot contradict it.

Though between science and common sense there exist dissimilarities we must not (and will not) overlook, the strong similarities between them establish for us a point of departure. Seeking to understand science, we begin by trying to understand the nature of common sense. Thus proceeding, we simply follow the counsel of Einstein, the subtle theoretician whose views have been widely regarded as a complete repudiation of common sense.

The whole of science is nothing more than a refinement of everyday thinking. It is for this reason that the critical thinking of the physicist cannot possibly be restricted to the examination of the concepts of his own specific field. He cannot proceed without considering critically a much more difficult problem, the problem of analyzing the nature of everyday thinking. 
Common sense and prevision. Why do we speak of common sense? First, because it is possessed and employed in much the same manner by the generality of mankind. The sage has uncommon sense: his less gifted contemporaries have a sense common to all. Second, because it is unexalted, untheoretical; it is down-to-earth, actively concerned with the business of living as distinct from the purposes or values or meaning of living. We judge that a man lacks common sense when he fails to adopt some course of action we feel he should recognize as most likely to yield him the ends he seeks-when he fails to foresee, as he should, the future consequences of present action. To lack common sense is to lack a previsional capacity.

Can anything as mundane as common sense lay claim to such an exalted capacity? On what basis can the future possibly be foreseen? Past experience offers the only possible basis. Common sense must so work upon the experience of the past that it is made available for anticipation of the future. The person lacking common sense may fail to detect any recognizable pattern in his past experience, and so lack all basis for extrapolation into the future; or he may fail to recognize the recurrence of situations that have been effectively dealt with in terms of some particular pattern. In either case he fails to learn from experience. Capacity for such learning is a cherished faculty of man; common sense represents the exercise of that faculty.

We do not recognize common sense in the lower animals. In them "instinct," various tropisms, and conditioned behavior serve to produce activity without-we may suppose-any prevision of the consequences of that response. But if the species is to survive, the instinctive responses must for the most part be appropriate responses, giving the appearance of a sound foresight. Meyerson says:

. . . foresight is indispensable for action. Now action for any organism of the animal kingdom is an absolute necessity. Surrounded by hostile nature it must act, it must foresee, if it wishes to live. "All life, all action," says Fouillée, "is a conscious or an unconscious divining. Divine or you will be devoured."

In the higher animals the nonreflective responses give way to activity more closely controlled by what, in the apes, approaches that which passes as common sense in man. A better contrived, more subtly varied response to the situations of everyday life then becomes possible. Given sound common sense, says Santayana, 
. . each moment of experience becomes consequential and prophetic of the rest. The calm places in life are filled with power and its spasms with resource.

Instinct in the animal has behind it a deep elemental drive-and well it may, for on it depends the survival of the organism and its species. As the control of action by instinct gives way to control by common sense, the latter assumes immense responsibilities, and thus becomes the focus of acute anxieties. In both animal and human subjects, systematic frustration of reasonable expectations produces neurotic disturbances. Such disturbances may be provoked even by exposure to circumstances that seem unreasonably to deny the cherished possibility of finding in past experience pointers toward the future. For example, Hebb notes that:

Pavlov . . . taught the dog that food would be given following the sight of one object, and not following that of another. No punishment was given if the dog failed to discriminate between them. The objects were made more and more alike until, after several days of failing to discriminate, the dog's behavior changed, suddenly. Instead of coming eagerly to the experimental room the dog struggled to avoid it; instead of standing quietly in the apparatus, waiting for the next signal to appear, he struggled and howled.

The deep-seated drive so displayed is presumably carried over into the active purposefulness of the science which, whenever it succeeds in descrying an order in past experience, makes us master of a future shorn of its terrors precisely in the degree that it can be fettered by chains of predictions.

\section{The Organization of Experience}

The individual's experience is varied and chaotic: how can he possibly give it a systematic order? The individual's experience is of certain particular situations of the past: how can he possibly extrapolate from them to inevitably non-identical situations of the present (and future)? The individual's experience is personal, subjective: how can he possibly arrive at the body of common sense shared by all? To facilitate discussion of these, and other, questions, I shall consider the organization of experience as a sequence of "stages"; but I would emphasize at the outset that these are not pro- 
posed as stages in the operations of the senses and the brain, but simply as artifices of discussion: i.e., steps in the abstract analysis of what is in practice an irreducibly integral (and ordinarily nonreflective) process in which each "stage" presupposes or is presupposed by others.

\section{FROM STIMULI TO CONSTRUCTS}

Merely to see an object is no mean feat of organization. The measure of the achievement is indicated by the results of corneal transplantation, giving sight to adults blind from birth. Young tells us:

The patient on opening his eyes for the first time . . . reports only a spinning mass of lights and colors. He proves to be quite unable to pick out objects by sight,. . . His brain has not been trained in the rules of seeing, does not know which features are significant and useful for naming objects and conducting life. We are not conscious that there are any such rules; we think that we see, as we say, "naturally." But we have in fact learned a whole set of rules during childhood.

At least a month passes before the subjects can recognize even a few objects, and “... if sufficiently encouraged they may after some years develop a full visual life and be able even to read." Making all due allowance for the greater co-ordinative plasticity of infancy, still we cannot but recognize that "seeing"-no simple passive reception of an unequivocal signal-is a formulative process in which the receiver plays an active role.

This conclusion is reinforced by the results of physiologic research. The eye contains some one hundred million separate receptors. To "see" a thing must then demand some work of construction from the myriad atomic stimuli. But even to speak of "construction from atomic stimuli" is an oversimplification. Such stimuli are separately unknown to us; they are not facts but hypotheses. Weyl argues that:

Consciousness reacts with an entirety that is not merely a mosaic composed of sensations; on the contrary, these so-called sensual data are a subsequent abstraction. The assertion, that they alone are actually given and the rest is derivative, is not a description that care-

"I must take cognizance of these results for, as becomes plain in Chapter XII, I wish to maintain a realist position. 
fully pictures what is given in its full complexity, but rather a realistic theory arising from the realistic conviction that "only sensations can really be given." Nevertheless, one may believe in a subconscious weaving of the whole pattern out of such elements called pure sensations; . . .

We began by asking how common sense organizes the data of experience. We see now that the term "experierice" itself conceals complexities. At the very least, the "experience" with which common sense comes to grips already bears the stamp of a receptor apparatus that-as Langer remarks-contributes actively to a rudimentary organization of what is received.

A tendency to organize the sensory field into groups and patterns of sense-data, to perceive forms rather than a flux of light-impressions, seems to be inherent in our receptor apparatus just as much as in the higher nervous centers with which we do arithmetic and logic. But this unconscious appreciation of forms is the primitive root of all abstraction, which in turn is the keynote of rationality; so it appears that the conditions for rationality lie deep in our pure animal experience -in our power of perceiving, in the elementary functions of our eyes and ears and fingers. Mental life begins with our mere physiological constitution.

Provided with physiologically similar sensory equipment, the higher apes seem to see objects somewhat as we do. Judging from their actions, so do far lower animals; for example, like ours, their vision singles out for attention any trace of movement in a complex but otherwise static visual field. Ability to see "moving objects"-objects to be pursued, to be eaten, or objects to flee or to hide from-has an obvious survival value. Perhaps then, by way of natural selection, capacity to see "objects" comes to be a built-in feature of the sensory organs of species successful in the struggle for life. Shaping us for this particular kind of perception, our animal history thus underlies the activity of common sense and science alike.

Observing. What part of the purely physiological organization of experience takes place in the sensory organ, and what part in the brain, is unclear. Some of this organization, e.g., the figure-ground distinction, seems to be wholly spontaneous and unlearned. But we do know that visual perception involves, in addition to the immediate locale of the sense termini, a rather large part of the brain. Hebb moreover tells us: 
Electrophysiology of the central nervous system indicates ... that the brain is continuously active, in all its parts, and an afferent excitation must be superimposed on an already existent excitation.

Thus any and all "automatic" organizational activity will merge into further activity, at the fringe of consciousness, in which learning is involved.

We "learn" to use our senses-e.g., to keep our eyes in focus, to run them along the contours that define an object. Beyond this literal aspect of learning lies an equally important figurative aspect. In infancy we learn not only control of the musculature of the eye but also the forms or patterns of "things" and "situations." To conceive this learning is difficult: it all takes place without conscious volition in a period lost in the haze of early childhood. Ordinarily we become aware of the function of the learned forms only in cases involving new patterns learned in maturity and, more strikingly, in cases (of illusion) in which the taken-for-granted forms prove insufficient or actively misleading. But our "knowing" always affects our "seeing." We learn not only how to see but also what to see; often we simply don't see anything for which our learning has not, in some sense, prepared us. The seaman notes small details in the condition of sea and sky that pass unnoticed by the landlubber. Usually the seaman's vision is no sharper, but he observes, as significant, points the landlubber could but does not see, because to him they are inconsequential features of the visual field-taken in by the eye but not by the brain. The seaman possesses a "mental image" (Gestalt, neural pattern, or what you will) in or against which certain details stand out in bold relief. Consider bird-watching. The old hand's claim to make a valid identification on the basis of a fleeting glimpse astonishes the novice, but what stuns him is that the old hand seems to see so much more. Of course the old hand sees more: he is not passively seeing but actively looking, looking for significant similarities and differences in the bird before his eyes and one or several images carried in his "mind's eye."

The ancients supposed that seeing involved a projection of rays from observer to object seen. Today we conceive seeing as the transmission or reflection of rays from object to eye. False as a physical mechanism, the ancient view nonetheless symbolizes an important truth. Seeing involves a merging of something from the object, "outside," with something contributed by the viewer, from inside. Brain 
and eye collaborate in seeing. What we claim to perceive depends no more on the sensitivity of the eye than on the conceptual inventory deposited in the brain. Nowhere is this more apparent than in experiments like those of which Polanyi gives us the following perceptive analysis:

Ames and his school have shown that when a ball set against a featureless background is inflated, it is seen as if it retained its size and was coming nearer. This illusion seems to be due to the fact that in this case we accommodate our eyes to a closer range, even though in consequence the object gets out of focus. . . . These defects of the quality and position of our retinal images are accepted here by the eye, in the urge to satisfy the more pressing requirement of seeing the object behave in a reasonable way. Since tennis balls are not known to blow themselves up to the size of footballs, a ball which does so must be seen as approaching us, even though in shaping this sensation the eye must override standards of correctness which it would otherwise accept as binding.

The rule that we follow in shaping the sight of the inflated ball is one that we taught ourselves as babies, when we first experimented with approaching a rattle to our eyes and moving it away again. We had to choose then between seeing the rattle swelling up and shrinking alternately, or seeing it change its distance while retaining its size, and we adopted the latter assumption. By this way of seeing things we eventually constructed a universal interpretative framework that assumes the ubiquitous existence of objects, retaining their sizes and shapes when seen at different distances and from different angles, and their color and brightness when seen under varying illuminations. ... .

In a larger perspective, the present experience of seeing the inflated ball come nearer to our eyes appears merely as the last of a lifelong chain of experiences encountered and shaped by us, to each of which we reacted to make sense of it as best we could, and which are now all subsidiarily effective in the shaping and comprehension of our present experience.

Constructs. What we may call "bare experience" already confronts us with an inseparable fusion of perceptual and conceptual elements. For us "the given" is perhaps formed from sensory stimuli, or percepts; but formed in the pattern of a supposed general class, which is a concept. What enters consciousness Margenau calls a construct. 
We perceive a complex of colors, shapes, motions, mingled with fragrance and perhaps tactile data, all suffused with an awareness of "out there"; the whole experience is summed up in the declaration: This is a flower. . . The postulation of an external object is the first phase of the cognitive act.

Margenau is careful to emphasize that the spontaneous organization yielding this vision of things

involves more than integrations: it involves construction, construction in accordance with rules. Objectivity emerges as a result of this procedure; . . .

Objectivity? To be sure, we agree on what we "see" not because the sensory stimuli are precisely the same-they cannot be-but because of the normative function of the conceptual patterns in terms of which our constructions are made. The differences in the stimuli -due, for example, to individual differences in sensitivity, and to the necessarily different perspectives of two simultaneous observers of the same thing-drop out as the construct is formed in a conceptual pattern used by all observers. The bare possibility of a common sense depends on common acceptance of the same fundamental group of mobilizing forms or patterns. The spontaneous interaction of percepts and concepts is here enormously advantageous.

Objectivity? Certainly not! What emerges from construction is at most some degree of impersonality. The constructs involve a large subjective (conceptual) component passing undetected because it is common to all. "Pure observation," "naked fact," are no less hypothetical than "atomic stimuli." Thus, as Mill emphasizes, the objects of common sense-objects also for science-are far from purely objective.

. . . in almost every act of our perceiving faculties, observation and inference are intimately blended. What we are said to observe is usually a compound result, of which one-tenth may be observation, and the remaining nine-tenths inference.

Thus we constantly infer the presence of massive solid objects (e.g., tables, chairs, other people) from sensory data presenting neither solidity nor massiveness. Generally such inferences are sound and extremely helpful. But the spontaneous compounding of "one-tenth observation" with "nine-tenths inference" is also potentially hazardous-for a reason clearly suggested by a comment of Hebb's: 
There is plenty of evidence in children's drawings, and in adult errors in perspective drawing, to show that a person looking at an object thinks he sees more of it than he does. What he knows [or thinks he knows] about the object appears in his drawing, as well as what is visible at the moment; and the significant fact is that neither child nor adult can usually say where his drawing departs from what is actually presented to the sense organ.

Unable fully to distinguish observation from inference, man has found it all too easy to believe that, for example, the fixity of the earth is obviously a "given fact."

\section{FROM CONSTRUCTS TO CONCEPTS}

Following the organization of "experience," we have passed from the sensory organ to the brain-from the notion of a bare percept to that of a construct already presupposing the organization given by a concept. From early childhood we begin to acquire the multitude of concepts necessary for the business of living. For example, the child learns to associate the concept "mother," and the word symbolizing it, with a "particular person." But in what way a particular person? Not one who always acts the same: sometimes she does things that please, sometimes things that hurt; sometimes her voice is soft and loving, sometimes harsh and commanding. She does not always look the same. She does not always wear the same dress, or even always wear dresses; her coiffure, her make-up, her expression all vary. And all these variations in the child's experience of "mother" must be $s u b$ tracted, allowed for, or dismissed as irrelevant in the formation of the concept. An addition is made when he grasps that underlying the "superficial variations" in the experience of mother there is an unchanging "something." To achieve even so primitive a concept, the child must perform a feat of abstraction and synthesis that Hebb notes is difficult enough to baffle an infant ape.

Dr. R. and Mr. T. are regular attendants in a chimpanzee nursery; the infants are attached to both, and eagerly welcome being picked up by either. Now, in full sight of the infants, Dr. R. puts on Mr. T.'s coat. At once he evokes fear reactions identical with those made to a stranger, and just as strong.

Meyerson tells us that all science represents the effort to discover, or create, identity in experience. That pursuit is already reflected in 
the child's formation of concepts. Piaget emphasizes the importance of concepts of "conservation" in the elaboration of the child's vision of the world. Only by way of his growing recognition of invariants, and invariance, does he come at last to find his world intelligible. Lorenz identifies the essence of Gestalt perception as
... the single function of extricating the essential constant factor by abstracting from the inessential variable sensory data. The differ- entiation of this function attains an amazing development in the serv- ice of shape constancy and it needs only to be driven one little step further to make possible an absolutely new operation miraculously analogous to the formation of abstract, generic concepts.

Whether or not one accepts the Gestalt theory of "extrication," of the constant factor, one recognizes how very natural will be the emergence and refinement of functional capacity for this discrimination in creatures evolved under the pressure of natural selection. The immense value of that "one little step further" is amply suggested by Bartlett's analysis of the choice and arrangement of evidence by normal subjects placed in problem-solving situations. He strongly emphasizes the leading part played

. . . by the detection of points of agreement. It is well known that points of agreement are inherently less easy to detect than points of difference. Perhaps the most important distinction between the two, from our present viewpoint, is that the detection of differences alone leads nowhere in particular in a positive sense, but the detection of agreement may. Thus, if one instance is observed to differ in some way from another, it may perhaps be said that something already established about the one is not applicable to the other; but if two instances are observed to agree in some way, it may possibly be implied that something already established about the one is applicable to the other. It seems fairly certain that, in a cognitive sense, all advance of knowledge comes by using agreements to get a move on, so to speak, and then using differences to keep the move within limits, and to show where a new direction of move becomes necessary.

The normative factors. In Lorenz' view, the constant factor in experience is not so much "given to" as "computed by" the organism. In some degree each must create concepts for himself-and this may seem to raise a serious problem. How can we all come, as we do, to a common understanding of the concepts of common sense? Normative influences plainly are at work. 
We have in common a biological heritage. Moreover, the experiences of early childhood, in the context of which each individual begins to form his stock of concepts, are far more uniform for all of us than are the experiences of any later period. Then too, the things and situations called to our attention in childhood-and the way they are presented to us-are in large part determined by a comparatively uniform cultural tradition. More specifically our language, by virture of the resources it offers and fails to offer, must, as Whorf observes, condition the species of concepts we form and the way we use them.

We cut nature up, organize it into concepts, and ascribe significances as we do, largely because we are parties to an agreement to organize it in this way-an agreement that holds throughout our speech community and is codified in the patterns of our language.

Polanyi offers a handsome illustration of how language mediates the interplay of percepts and concepts:

Think of a medical student attending a course in the X-ray diagnosis of pulmonary diseases. He watches in a darkened room shadowy traces on a flourescent screen placed against a patient's chest, and hears the radiologist commenting to his assistants, in technical language, on the significant features of these shadows. At first the student is completely puzzled. For he can see in the X-ray picture of a chest only the shadows of the heart and the ribs, with a few spidery blotches between them. The experts seem to be romancing about figments of their imagination; he can see nothing that they are talking about. Then as he goes on listening for a few weeks, looking carefully at ever new pictures of different cases, a tentative understanding will dawn on him; he will gradually forget about the ribs and begin to see the lungs. And eventually, if he perseveres intelligently, a rich panorama of significant details will be revealed to him: . . . He has entered a new world. He still sees only a fraction of what the experts can see, but the pictures are definitely making sense now and so do most of the comments made on them. He is about to grasp what he is being taught; it has clicked. Thus, at the very moment when he has learned the language of pulmonary radiology, the student will also have learned to understand pulmonary radiograms. The two can only happen together. Both halves of the problem set to us by an unintelligible text, referring to an unintelligible subject, jointly guide our efforts to solve them, and they are solved eventually together by dis- 
covering a conception which comprises a joint understanding of both the words and the things.

Whatever the respective weights of the various normative factors -biological, sociological, cultural, linguistic-together they are powerful in promoting common understanding of the concepts of common sense. Such understanding is then entirely compatible with the individual creative intuition I hold requisite in all concept-formation.

Varieties of concepts. The concepts of common sense are manifold. Consider examples of three varieties we shall find strongly represented among the concepts of science. First are those which envision as an element of identity an unchanging thing underlying, and productive of, our changing percepts. Langer writes:
A little reflection shows us that, since no experience occurs more than once, so-called "repeated" experiences are really analogous oc- currences, all fitting a form that was abstracted on the first occasion. ... I believe .. . that we promptly and unconsciously abstract a form from each sensory experience, and use this form to conceive the experience as a whole, as a "thing."

In common sense such thing-concepts have generally a quite direct relation to the perceptions that evoke them; but the situation is not uncomplicated. Surely the concept "mother" derives comparatively directly from a set of visual, auditory, and tactile stimuli. But no simple fusion of the sensory elements yields the concept "mother"; no particular construct of mother quite corresponds to the concept "mother." Observe also that the simple common-sense concepts already display a spectrum of unobservability continuous with the much extended spectrum we remark in the thing-concepts of science. Even "mother" is only intermittently present in the sensory field, and the very young child may believe that "mother" is annihilated when he sleeps and recreated by his cries on awakening; but he soon acquires full belief in the unobserved, and for him unobservable, continuous existence of "mother."

Consider now, as a second variety of common-sense concepts, those class-concepts which group together things so much alike in "important" qualities that we can ignore the respects in which they differ. The whole possibility of learning from experience is tied up with the development of a suitably discriminating group of classifi- 
catory concepts that teach us how to find in nonidentical things a significant identity in type. Mill writes:

We compare phenomena with each other to get the conception, and we then compare those and other phenomena with the conception. . . . and if it [a particular instance] agrees with that general conception, we include it in the class. The conception becomes the type of comparison.

There is this rattle-with its own color, shape, weight, and sound. There is this other rattle, with its own distinct set of qualities. And there is the general concept "rattle." A member of the class must be, in some degree, graspable and shakable; and it must give a "rattling sound," though this may vary greatly in volume and timbre. In forming the class some of the most striking qualities (e.g., color and shape) are adjudged irrelevant, and even the key qualities are permitted to vary within broad limits. The discrimination so expressed represents appreciation of the function of rattles.

Function seems generally decisive in our understanding of classificatory concepts, both in common sense and in science. Oftenthough we all understand a concept in much the same way-we can find no specific criterion or group of criteria that establishes the class. In such cases we deal far less with rigid specifications than with a general pattern. Failure to fit the pattern in some respects is disregarded if a good fit obtains in other respects that most concern us at the moment. Our grasp of meaning is strengthened by our sense of purpose-a point stated in its broadest application by Popper:

. . any two things which are from one point of view similar may be dissimilar from another point of view. Generally, similarity, and with it repetition, always presuppose the adoption of a point of vicw: some similarities or repetitions will strike us if we are interested in one problem, and others if we are interested in another problem. . . . points of view, or interests, or expectations, are logically prior, as weli as temporally (or causally or psychologically) prior, to repetition.

Each of a third variety of common-sense concepts highlights some one attribute of a great many things. Though often unlike in all other respects, these things can meaningfully be compared with respect to this one "property." Such quality-concepts, abundantly represented in common sense, express recognition that for a given purpose a certain quality is of overriding importance. In getting the first 
line across a gorge the length of the available cordage is the factor of primary concern; secondary attention may be given to weight and strength, but the composition of the line is of no concern whatever. The pilot of a plane laboring in a storm will feel a primary concern for the weight of his cargo; its shape does not interest him, though its value will cause him longer to defer jettisoning gold than sand.

Ultimately, quality-concepts are adjectival, as when we speak of a weighty object or a long rope. But, once recognized as useful, they may be transmuted into nouns, like weight and length. Such a "property of matter" is a characterization of something in relation to a context of observation. If we take the context for granted, as invariable if not invariant, we may usefully regard the property as an inherent quality of the something observed. Thus, setting out from the conception of "moving bodies," we come ultimately to the study of "motion" as such. However, an element of danger always attends such transmutation. Having rendered "loving" into "love," "honorable" into "honor," "thinking" into "thought" and even "mind," the philosopher may entice us into pursuit of apparently meaningful but utterly empty questions: $e . g$., what is the nature of that thing called mind? The man of common sense himself often falls victim of this misconception of adjectives as nouns. Even the scientist has not been immune: e.g., the concept of light as an undulatory phenomenon gives rise to the concept of a medium of undulations-the luminiferous ether.

The tool function of concepts. Concepts are not found as such in nature; they are evoked in the human mind by nature. Experience cannot warrant concepts "true" or "false," only appropriate or inappropriate. Concepts prove themselves as tools prove themselves. James writes (in italics) that:

. . our fundamental ways of thinking about things are discoveries of exceedingly remote ancestors, which have been able to preserve themselves throughout the experience of all subsequent time.

In my instantaneous visual field I register the construct I call "this man." The characteristics of "this man" stand out from the pattern of the concept "man." I then relate my experience of this man today to my experience of that man yesterday and, within limits, predict my experience of this or that man tomorrow. Ability to make such predictions is enormously valuable, and the concepts conveying such 
predictive capacity preserve themselves through ages of human thinking.

The concept "man" relates yesterday's experience of one man to today's experience of a second and to tomorrow's predicted experience of a third. But how? The concept shows me an element of identity in all the men, but by itself offers nothing intrinsically useful for prediction. "Relate" then must have some further meaning. Effective organization and prediction of experience implies not merely concepts but relations among concepts. In my mind the concept "man" is associated with a group of other concepts referring to many aspects of "human behavior." Through such associations the concept "man" takes on the full measure of its meaningfulness. "Man" may be old or young, strong or weak, tall or short, white, black, red, or yellow-but by and large "man" shows "human behavior." Organized by the concepts of human behavior, my past experience of "man" permits me to guide my dealings with this man now by means of forecasts of the various results likely to be produced by alternative courses of action. Only by way of the confirmation of many of those forecasts can the appropriateness of my concepts be demonstrated and their denotations become firmly established. Our selection and understanding of concepts is required for, and inversely determined by, the statement of the relations we seek.

\section{FROM CONCEPTS TO COLLIGATIVE RELATIONS}

What I shall call colligative relations so link together certain elements of our past experience that the appearance of such elements in future experience is made subject to prediction. Typical colligative relations are: "The burned child dreads the fire" or "Water seeks its own level” or Boyle's law-“At constant temperature and with constant quantity of gas the product of pressure and volume is a constant." A scientific law is often a colligative relation, as Jeffreys indicates:

The test of a scientific law is its capacity to account for sensations already recorded, and its interest lies largely in its capacity to predict new ones.

The adjective colligative is essentially neutral and descriptive. Literally, to colligate is to bind together. Every relation must represent some kind of binding-together, and to this extent the adjective 
is redundant. Yet it serves to emphasize the several senses in which these relations represent a binding-together. Thus, for example, they represent a binding-together in time: based on the past, they are used in the present for prediction of the future. Also, these relations associate certain concepts with each other, and thereby reaffiliate the several elements of experience to which they refer. We link "pressure" and "volume," or "water" and "level," or "child who has been burned" and "fire." Most significantly, in colligative relations we find perceptual and conceptual elements bound together in a balance unduly weighted one way or the other when such relations are termed "phenomenologic" or, contrarily, "causal."

Phenomenologic means "descriptive of actual phenomena with avoidance of all interpretation, explanation, and evaluation." A colligative relation cannot so be described: it represents both much more and much less than a "description of actual phenomena." Though I have observed very few burned children, I make a general statement about all such creatures in all times and places. Here I go far beyond what I have observed. But also I say much less than I have observed. The concepts figuring in the general statement single out only certain "significant" aspects of a complex total experience, other aspects of which pass unmentioned, as "irrelevant." But irrelevance is not "given"; it involves a judgment, an "evaluation."

In Chapters II and V we shall examine in detail the multiple interpretations, explanations, and evaluations we must go through to reach a "purely scientific" relation such as Boyle's law. Only the ghost of actual observations survives in the final statement of the law. To describe such a relation as phenomenologic is folly; to describe it as causal, equal folly. We may feel tempted to say that the doubling of the pressure is the cause of the halving of the volume of a confined gas at constant temperature. We might better confine ourselves to: If the pressure on a confined gas at constant temperature is doubled, then its volume will be halved. Determinism is taken for granted, and a causal connection is implied. But the assignment of causes is here an act of supererogation: the acausal "if ... then ..." statement alone discharges the predictive function of Boyle's law.

The imputation of causes is more apparent but logically no less superfluous in the colligative relations of common sense. Perhaps the burned child's "dread" is the cause of his subsequent avoidance of fire; but such dread is an hypothesis, not an observation. The useful- 
ness of the relation has nothing to do with "dread" construed as cause. If a child has suffered a burn, then he is not thereafter likely to be found in the immediate vicinity of fires. The original burning and the subsequent avoidance of fires are the observational terms to which the relation gives order, and through which it yields predictions. By supplying an understandable link between the two observational terms, the hypothesis of "dread" may serve a mnemonic function-it also permits terse statement of this common-sense maxim. The same two values are conveyed by the word "seeks" in the maxim: "Water seeks its own level." But the function of the colligative relation is entirely independent of the imputation of purpose, as it is of cause.

Whatever the form of their verbal statement, colligative relations have value for common sense if reducible to the form: "If $A$, then B." We predict the future situation B on the basis of past or present observation that situation A obtains. Purposive behavior becomes possible: wishing to bring about situation B we work to realize situation A. Desiring to halve the volume of a gas, I seek to double the pressure on it. The conceptual formulation of the relation renders it both brief and general, terse and pithy, abstract and broadly applicable. The relation constitutes communicable knowledge, where the encyclopedic enumeration required for statement of a purely phenomenologic relation would be, practically, incommunicable.

Denotation. "We see "things," no two exactly alike, and arrive at concepts of classes of things essentially alike. Observing things in various situations we pass, by a further creative act, to colligative relations the extreme abstractness of which is generally masked by our familiarity with them. However, if such relations are to have the slightest value to a common sense, usable by all, their conceptual terms must meet one essential criterion: The concepts that figure in the colligative relations of common sense (and science) must have associated with them reasonably unequivocal experiential denotations. Only by virtue of such denotations can we get back from the abstraction of the conceptual statement to the "hard realities" of purposive action to produce a predictable end. The statement that water

"Following Webster, I will use denotation to signify "the class of individuals or instances falling under a conception or named by a term. . . . The denotation of a word is its actual meaning; its connotation that which it suggests or implies in addition to its actual meaning." 
seeks its own level becomes meaningful and useful only if I can recognize "water" and the behavior known as "seeking its own level."

How precise are these denotations? Their precision is the resultant of a complex process in which desire for reliability and desire for generality (and simplicity) pull in opposite directions. Guided by our past experience we may elect to spell out the denotations in such detail that the risk of misunderstanding is minimized. But by that very act we also minimize the concepts' applicability: the things and situations of the present (and future) will never precisely reproduce those of the past. The whole possibility of achieving a generally useful colligative relation thus hinges on our willingness not to specify too rigidly the denotations of its conceptual terms.

What do I take to be the denotation of "water"? Sometimes clear, sometimes turbid; sometimes "hard," sometimes "soft"; limpid or colored; bland, salty, or bitter; warm or cold; odorless or pungent; potable or contaminated; liquid, solid, or gaseous-how equivocal can a specification be? Yet men of common sense ordinarily agree on what is to be classified as "water." As already noted, the existence of a purpose for which the classification is made sharpens discrimination: it establishes the crucial functional characteristics for all who share that purpose. In any matter of seeking its own level, "water" need only be a uniformly dense liquid of modest viscositynothing else "counts." For thirsty travelers in the Mohave desert a particular solid will be happily accepted as "water"; but for the captain of a Greenland patrol ship a large block of that particular solid is not "water" but "menace." The concept remains broadly applicable because its denotation rests imprecise, sharpened only as required by the "common sense" of those who, for one purpose or another, must deal with "water."

In common sense the tension between the desire for precision and the desire for generality is most often resolved in favor of generality. The number of concepts and relations-with which common sense can work effectively is, we shall find, severely restricted. Each concept, each relation, must then render maximum service; and a good deal will be sacrificed in the way of reliability to obtain maximum generality. We all share Poincarés feeling that: "It is far better to foresee even without certainty than not to foresee at all." Moreover, in the affairs of everyday life, with which common sense must deal, we constantly meet multiple special contingencies that no colligative 
relation-even a specialized one-could handle perfectly. We say "The burned child dreads the fire," but some children who have suffered a burn do not shun fires. Neither humans nor animals always shun the thing or situation of which they have had disagreeable experience. "It all depends"-on how disagreeable the experience, on the balance of estimated possibility of pleasure against estimated risk of pain, on the psychic state and background of the subject, and so forth. Even specifying all these, the predicted behavior would still sometimes fail to occur. Perfect predictability being unobtainable, we forego the clumsy (and, in the issue, not readily verifiable) specifications in favor of the brief and unadorned general statementadmittedly crude but perhaps statistically valid as a guide to action.

Earlier I suggested that colligative relations could be reduced to the form: "If A, then B." I was not altogether correct. The more accurate form is: "If A, then probably approximately B." Due to uncertainties in the conceptual denotations, we may err even in routine applications of a given maxim; and in atypical situations even the choice of the approximate maxim may become doubtful. The child who has learned a maxim of common sense has not thereby acquired the ability to use it successfully. Knowing it only as an abstraction, he must still acquire a "feel" for the concrete situations in which it can be used. Polanyi argues that:

. . . in all applications of a formalism to experience there is an indeterminacy involved, which must be resolved by the observer on the ground of unspecifiable criteria. Now we may say further that the process of applying language to things is also necessarily unformalized: that it is inarticulate. Denotation, then, is an art, . . .

Experience helps here; indeed, it is indispensable. The child fails to discriminate the situation in which he should look before he leaps from that in which he who hesitates is lost. The experienced battle commander shows his competence in just such discrimination.

\section{A FOURTH STAGE IN THE ORGANIZATION OF EXPERIENCE}

Through three mutually interacting stages we have passed from stimuli to constructs, from constructs to concepts, and from concepts to colligative relations. A multitude of such relations represents the achievement of common sense-a major achievement making pos- 
sible purposive and generally successful behavior in everyday affairs. But simply as an organization of experience this is still an incomplete achievement. Command of the bewildering multitude of colligative relations-apparently intrinsically individual, discrete, fragmentary-presents a superficially insoluble problem. To master such relations there seems no alternative to brute-force memorization, an expedient wasteful of time and effort and fundamentally limited by the memory's capacity. The problem is further exacerbated in that we must memorize not only relations but also the limits in range and reliability pertaining to each, and, beyond that, the manifold denotations that invest its conceptual terms with experiential relevance. The child requires years to master the relations of common sense; the apprentice requires years to master the relations of an empirical craft.

We observed earlier that concepts (words) mean nothing without relations (sentences), but Wittgenstein adds that "understanding a sentence means understanding a language." Why so? Whitehead has the answer:

There is not a sentence which adequately states its own meaning. There is always a background of presupposition which defies analysis by reason of its infinitude.

In common sense the major part of the background of presupposition is simply the world-view necessarily implicit in any language of common sense which, for example, takes entirely for granted certain "accredited" concepts of things, and the classes and qualities thereof. Gradually acquiring command of that theory, the child learns, and learns to use, the concepts and relations of common sense precisely as he comes to grasp the "nature" of the world of common sense. Similarly, the apprentice comes to be a master carpenter not simply by memorization and the acquisition of physical dexterity, but when he has grasped the "nature" of wood. With this insight he can pass beyond memorization (of the relations describing the working and structural employments of wood) to something approaching comprehension of how the various relations arise.

We find two major genera in the concepts of common sense (as in those of science). The first and larger group contains the indicative concepts, with relatively clear denotations, which figure prominently 
in colligative relations. A second group, of more abstract explicative concepts, sketches the "causes" and other terms by means of which we seek to grasp the relations. In principle only the indicative concepts are directly involved in making predictions-necessarily so since, often, explicative concepts (perhaps figuring "unobservables") do not refer to overt, recognizable things and situations. In practice both groups of concepts are (and probably must be) used; the second helping us to gain command of relations that function in terms of the first. In common sense even loose and imprecise anthropomorphic concepts can offer a systematization highly effective as a mnemonic device. Personifying the things of which we would treat, we invest them with desires and tendencies sufficiently like our own that-understanding what our action would be-we easily remember theirs. The child "dreads," water "seeks," nature "abhors," etc. We grasp the relation because we seem to grasp the how of its production.

In common sense the fourth stage in the organization of experience never becomes fully explicit: the formation of a world-view is not, after all, the business of common sense. Often little more than a vague metaphor, the common-sense view of the world leaves a great deal to the judgment and experience of the individual, to his "feeling for the situation." Much has still to be memorized because there remains much that, if "explained," is not yet truly rationalized. The comparatively inchoate body of common sense contains myriad relations not merely disconnected but as downright contradictory as are the injunctions "look before you leap" and "he who hesitates is lost."

Science seeks to resolve, or suppress, such contradictions by a conscious effort mutually to adjust and to unite its myriad relations in a fully explicit, comprehensive, rational order. No longer content to possess Boyle's law, we wish to see Boyle's law in rational connection with a number of other colligative relations describing empirical regularities in the behavior of gases. The kinetic theory provides just such a connection. The several relations now reappear as deductions from the few axioms postulated by the theory. In this dramatically new fourth stage in the organization of experience we pass on from colligative relations ("laws") to postulational systems ("theories"), and so achieve the order Einstein considers characteristically that of science: 
The aim of science is, on the one hand, a comprehension, as complete as possible, of the connection between the sense experiences in their totality, and, on the other hand, the accomplishment of this aim by the use of a minimum of primary concepts and relations. . . .

Science concerns the totality of the primary concepts, i.e., concepts directly connected with sense experiences, and theorems connecting them. In its first stage of development, science does not contain anything else. Our everyday thinking is satisfied on the whole with this level. Such a state of affairs cannot, however, satisfy a spirit which is really scientifically minded; because the totality of concepts and relations obtained in this manner is utterly lacking in logical unity. In order to supplement this deficiency, one invents a system poorer in concepts and relations, a system retaining [though usually in drastically modified form] the primary concepts and relations of the "first layer" as logically derived concepts and relations. This new "secondary system" pays for its higher logical unity by having . . . elementary concepts (concepts of the second layer), . . . which are no longer directly connected with complexes of sense experiences [that is, these are concepts that refer to "unobservables"]. Further striving for logical unity brings us to a tertiary system, . . .

Similarities in intent, presuppositions, and subject matter. The profound reorientation of its endeavor sharply differentiates science from common sense, but at least three similarities remain significant.

A primal biological drive seeks blindly to make past experience the determinant of present action that will succeed. Become selfconscious in common sense, this drive remains active even on those planes of lofty abstraction along which science extends itself as the continuation of common sense. "There is no sharp line between science and common sense," says Russell, "both involve expectations, but those resulting from science are more accurate." Meyerson urges that "whoever speaks of science speaks of predetermination," and in Sambursky's view this much was true even as far back as the Greeks, for he writes that

then, as now, the task of science was to systematize the sum total of our empirical knowledge in such a way as to make it possible to forecast future events.

This perfectly matches our definition of common sense, but an important redistribution of emphasis will soon be noted.

Science and common sense share certain "metaphysical presuppo- 
sitions." Down-to-earth common sense with metaphysical presuppositions? Of course, for as Morris Cohen observes:

... the common sense level is not one of primitive metaphysical innocence. The language of common sense is full of animistic, ancient, and scholastic metaphysics; . . .

In the infancy of science its presuppositions were perforce close kin to those of common sense. Science was not born in the void. It began somewhere, and with something. It set out from the position of common sense, and initially took for granted much that common sense assumed. The commitment is not irreversible-scientific experimentation began with crude workmen's tools, and these developed later into more suitable equipment; scientific thinking began with the modes of thought of common sense, and these too have undergone elaboration and modification. Even today, however, scientific thought must set out from the position of contemporary common sense. As Hebb suggests,

. . . all learning tends to utilize and build on any earlier learning, instead of replacing it (Mowrer, 1941), . . . the learning of the mature animal owes its efficiency to the slow and inefficient learning that has gone before, but may also be limited and canalized by it.

The science we learn in maturity is necessarily based on, referred to, and conditioned by the common sense we have learned and accepted in childhood.

Not all elements of experience are grist for the mills of either science or common sense. Both seek a communicable knowledge usefully applicable by all. Both then come naturally to limit their subject matter to experience that is regular enough to give some promise of a potentially predictable association of antecedents and consequences; reproducible, or at the very least not infrequently recurrent; and overt, open to and shared in by all human beings. The profound personal experiences that move us most-that we know best -are not then data acceptable to either science or common sense. They can be accepted only insofar as whatever seems irreducibly spontaneous is rejected (to meet the demand for regularity), whatever is absolutely unique is left out of account (to meet the demand for recurrence), and whatever is intrinsically "subjective" is omitted (to meet the demand for overtness). Whatever the consequences of 
such limitation in species of subject matter-and important consequences have recently been noted by Sherrington and Schrödingerthis is a limitation science shares with common sense.

Some differences: progressivism and impressiveness. In science we feel a wholly novel dynamic progressivism for which, anticipating the results of later discussion, I find at least three major sources. First, in science the fourth stage in the organization of experience provides an optimal deployment of what we already know in the search for what we do not yet know. Second, in the quest for new knowledge the development of refined physical devices often permits systematic experimentation to replace observation; and a new elaboration of mental devices is even more significant. The man of common sense disposes of a limited number of concepts, and must rely on these alone; the conceptual arsenal of the scientist is in constant rapid growth. Finding no existing concept appropriate, he is trained and prepared to make new concepts. Not bound by the resources of everyday language, he makes new words to represent his new concepts, and even, as required, new (mathematical) languages with structures different from that of everyday language. Third, science progresses simply because it is critical of its failures. Knowing the relations of common sense to be imperfect, we usually permit the survival even of relations that yield frequent unaccountable failures in prediction. Of a colligative relation of science-of a "law of nature" -we expect and demand more. Here an unexplained failure provokes energetic re-examination of the situation that, by revealing antecedent error, can open the way to subsequent progress.

Impressiveness is a further trait we find highly characteristic of modern science. We are amply impressed by the many accurate predictions it supplies, but overwhelmingly impressed by the sense of rational order it conveys to us. Like common sense, science must seek order as means to prediction. But in science the search for theoretical order takes on new urgency, and becomes so compelling that for most scientists prediction-no longer the end to which organization is the means-is rather the means of appraising progress toward the goal of rational organization. Why should order seem important as an end in itself? Finding ourselves able to organize our experience of the world, we feel ourselves able to understand the world of our experience. Some curious dualisms of meaning testify to the deeprootedness of this connection. To articulate means both "to join to- 
gether" and "to speak distinctly"; to comprehend, both "to include or contain" and "to understand"; coherence, "sticking together" and "consistency in reasoning."

There is explanation even in common sense. The tumbling of a mountain stream is for me "explained" by the simple colligative relation: water seeks its own level. Accepting the relation, I see the phenomenon as a necessary consequence of an existent initial state. In the same way, if in science I accept Boyle's law, I "understand" the change of volume consequent to the change of pressure on a gas. But now, in that fourth stage of organization characteristic of science, the myriad colligative relations and laws are themselves brought within a framework of explanation. Accepting the postulates of the kinetic theory, I find that they entail Boyle's law, and many others of its kind. The postulates so explain the relations, and the relations explain the phenomena. Rooted as it is in "vulgar common sense," science takes on the shape sketched by Einstein:

Science is the attempt to make the chaotic diversity of our senseexperience correspond to a logically uniform system of thought. In this system single experiences must be correlated with the theoretic structure in such a way that the resulting coordination is unique and convincing.

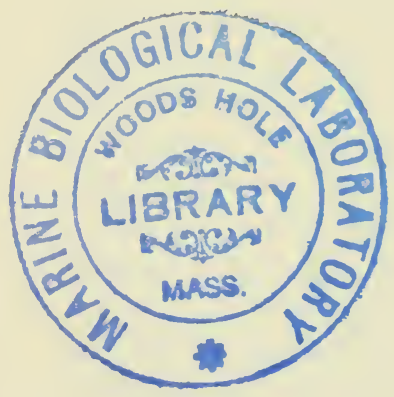




\section{CHAPTER II}

\section{Science (and Common Sense)}

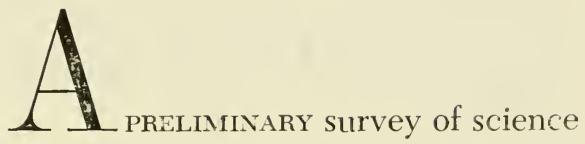

will now be made in terms suggested by its comparison with our reference standard, common sense.

\section{SOME "METAPHYSICAL PRINCIPLES" OF SCIENCE}

Science with "metaphysical principles"? But how else can I describe general ideas that go far beyond the warranty of experience and that are, nevertheless, stubbornly maintained even in the face of apparently contradictory experience?

The real world. Born comments on

. . Man's need to believe in a real external world, independent of him and permanent, and his ability to mistrust his sensations in order to maintain this belief.

The extraordinary success of the common sense (and the commonsense language) that consistently treats physical objects as possessing "real existence" will not a little justify for the man in the street his belief in reality. But, as Born further observes,

The simple and unscientific man's belief in reality is fundamentally the same as that of the scientist.

The scientist's accreditation of the idea of a real world 'remains unshaken even by the rise of quantum mechanics. Bohr holds to be a fundamental revision of our point of view the realization that: 
. . . a close connection exists between the failure of our forms of perception, which is founded on the impossibility of a strict separation of phenomena and means of observation, and the general limits of man's capacity to create concepts, which have their roots in our differentiation between subject and object.

This being conceded, Rosenfeld, not the least doctrinaire among those who count themselves Bohr's disciples, still maintains that:

. . . as far as quantum mechanics is concerned, I would say that it is impossible to understand it without assuming that there is an external world which is independent of what we think and which is the ultimate origin of all our ideas. In that sense I absolutely reject the suggestion of present-day positivists about the subjectivity of our statements.

Determinism. If I accept the possibility of prediction-whether by common-sense relation or scientific law-I assume determinism. If my past experience teaches me that state $A$ is followed by state $B$, then, observing a present occurrence of state A, I can predict the future production of state B if and only if I assume the same conditions are succeeded always, or at least statistically, by the same results. Prediction often proving possible, the assumption is in a measure justified. But even when prediction fails, rather than question the assumption of determinism on which all hope of prediction depends, I question instead the accuracy and completeness of my specification of the conditions necessary to define state A. At once I seek (and often I find) a relevant variable earlier overlooked.

Continuity. The creature endowed with instinct confronts the apparently chaotic disorder of nature with patterns of fixed responses. That species of creature can then survive only if nature embodies some general uniformity to which the fixed responses are, at least statistically, appropriate. Instinct presupposes the continuity of nature? However it may be with instinct, if I accept the possibility of prediction I presuppose, over and above the principle of determinism, a principle of continuity. Belief that state B will follow reliably on the attainment of state A means nothing if not coupled with confidence that state A (or a good approximation to it) will recur. We must then assume the "external world" continuous in time, in location, and in species. We assume that things behave here and 
now in much the way that "similar things" behaved there and then, and will again behave in other eras and places.

Dissolubility. Believing in the continuity of nature, I do not consider it an absolute continuum. Acknowledging myself a part of nature, I still consider myself separate from it. More significantly, we all conceive ours a world of distinct, if interacting, "objects" and "phenomena," in particular "situations" largely independent of the rest of the external world. All our labors of prediction assume this kind of dissolubility, assume each case measurably affected by a relatively small number of conditions-each state of a system adequately defined by a relatively small number of variables. Prediction would be impossible had we first to specify or determine the state of the entire universe. Confidently seeking colligative relations, common sense presupposes a principle of dissolubility. Seeking laws, so does science.

Emboldened by our predictive successes, both in science and in common sense, we make of the ideas of determinism, continuity, and dissolubility general principles that transcend all possibility of empirical justification. These "metaphysical" principles, together with a few others more peculiar to science, are examined in detail in Chapter VII. I broach them here because the sharing of these principles produces in both science and common sense certain shared criteria for the selection of "fit" subject matter.

\section{The Subject Matter of Science}

We expect recurrence in a world to which the principle of continuity applies, regularity in a world to which the principle of determinism applies, agreement of eyewitnesses in reports of an existing "external world." The principles we accept thus determine the selected elements of experience alone acceptable to us as facts. We are prepared to accept as facts only those elements of experience that are actually or potentially reproducible, regular enough to offer promise of determinist order, and concurrently reported by all "normal" observers.

\section{RECURRENCE AND REPRODUCIBILITY}

Guided by the principle of determinism, we expect that, in circumstances of adequate control, at least approximate reproduction of the conditions must yield at least approximate reproducibility of the 
results. Ordinarily we can achieve such control in the laboratory, ordinarily our results are reproducible, and ordinarily the laboratory worker demands reproducibility of anything he is prepared to treat as a result. Yet the demand for reproducibility is not an absolute one. Particularly as regards certain observational results, we may waive reproducibility in practice and accept the much weaker criterion of reproducibility in principle. We do not expect to be able to reproduce the observations reported for the fall of a particular meteorite. Even in the laboratory, studying cosmic rays we do not expect to be able to reproduce in our cloud chamber exactly the "nuclear event" previously photographed by ourselves or others. In both cases what we observe depends too heavily on variables beyond our control: on the nature, the relative velocity, and the angle of incidence of the intruding "particle." The "event" and the observations made of it are then irreproducible. Yet we accept those observations as facts because we conceive such events as recurrent, or reproducible in principle. Judging that we know the relevant conditions, we agree that the circumstances involved are very "special." And we maintain that-however improbable in practice-the event, and the observations made of it, would be reproduced if the special circumstances were reproduced in some second meteorite or nucleon.

Confronting a particular indentation in the sand-an indentation unique and irreproducible in detail-Robinson Crusoe sees it as a significant datum only because he at once affiliates it with the recurrent class "footprints in the sand." Much the same common-sense criterion of acceptability of data is active in the sciences. We accept data on irreproducible things and events when we judge that they belong with "objects of this class," "situations of this type," when we feel that other exemplars of such things and situations are known to us. Even when guided by the subtle sense of analogy with which we are so fortunately endowed, such feelings, such judgments, remain humanly fallible. Recognizing that sometimes we go astray in our judgments of recurrent type and class, we seek ever, by establishing experimental control of conditions, to achieve reproducibility in practice. We acknowledge the less secure criterion of recurrence only when we must. Yet often, even in science, we must. The astronomer accepts data on the unique trajectory of some particular meteor because meteors following analogous trajectories are recurrent. And he is likely to reject-as "observational error"-data indicating 
trajectories "too different" from those already observed. The physicist accepts data on a cosmic ray event he cannot reproduce because he feels it classifiable with other "events of the same kind." The palaeontologist accepts as datum a single jawbone or cranium, and seeks to reconstruct the entire animal involved. The fragment is unique, irreproducible, but he finds in it some filiation or likeness in type with other analogous remains. The sense of analogy renders the datum acceptable and the reconstruction possible.

Facts historical and scientific. The distinction drawn between the facts of history and the facts of science (and common sense) is far less sharp and significant than is supposed by one general school of thought-here represented by Langer's statement:

. . . nor is historical truth judged by the same criteria as the truth of scientific propositions. For to science, as Lord Russell once remarked in an academic seminar, "A miracle would not be important if it happened only once, or even very rarely"; but in history the point is to find out what did happen just once, what were the specific facts about a specific occasion.

As we have just seen, scientists do accept irreproducible data, "specific facts about a specific occasion," if they conceive the occasion to be recurrent. Russell and Langer notwithstanding, the acceptability of historical data seems to be judged by much the same criterion.

In the operations of historians and scientists there is a major difference in temporal reference-is this difference in itself decisive? Surely not! Seeking to reconstruct what happened in the past, the historian must and does take as data only presently available written reports, and the presently surviving physical remnants of antiquity; and part of the interest of his work (as of the scientist's) lies in its relevance to present or future situations somehow analogous to those of the past. When as scientist I consider my data, I work in part as historian. I, too, refer to events of the past: I consider my own experiments not in their present immediacy but in my memory and (more reliably) in my written laboratory records; I consult the presently available reports of my predecessors and contemporaries who have investigated analogous phenomena. My data may be experimentally reproducible, as the historian's are not; and perhaps this would be a decisive difference save that often as scientist I accept the weaker criterion of recurrence. 
Evaluating my data, I usually find them "consistent" internallyperhaps also in comparison with the data of some other observer(s) of the same or similar events. In any large array of data, however, I am likely to find one or a few that fail of consistency, either internally or externally. If, after long effort, I am unable to fit them into the class of the others, I create a new class for them-most often I dismiss them as "errors" or "aberrations." The historian, too, selects his data under the guidance of considerations of consistency. And his interest in his data, and his ability to work with them, are both product of his insight that-however apparently unique and irreproducible the events concerned-yet are they recurrent events, i.e., manifestations of "human nature," "economic determinism," "social stratification," “copyists' error," and so forth. In such classificatory judgments the historian guides himself as does the scientist. Either may err, and then the scientist's error may be the more readily rectifiable: often he can get a more abundant supply of data on "events of the same kind.” But the palaeontologist is a scientist who generally cannot, and Schliemann a historian who could and did.

The scientist finds the unique absolutely intractable, and thus rejects as data any findings he cannot envision as members of a recurrent class. The historian finds himself unable "to make anything of" events wholly unlike any others known to him; he, too, shuns the unique. With all due respect to Russell, modern historians rarely do treat of miracles. To the extent that they do so they dispel the uniqueness of miracles by attaching them to a class (e.g., instances of divine intervention in the affairs of men, suspensions of natural law, suspensions of human reason). But most nontheological historians are uneasy in writing of miracles because it is not plain that they are recurrent; because their proper classification is uncertain; because often there is but one historical account, and that of imperfect internal consistency, or several accounts with mutual consistency not wholly beyond reproach. The scientist's rejection of miracles as data fit for scientific study is founded on similar considerationsand they are the same considerations he would use routinely as, say, meteor-observer.

Uniqueness and identity. We seek general knowledge, general order, general predictability. This quest, frustrated in studies of the wholly unique, can prosper only as we find "sameness." We are then clearly well advised to insist that acceptable as subject matter are 
only those of the myriad data of experience that show, through recurrence, at least some minimum degree of sameness. One notable exception: if attested by concordant results of independent observers, a unique event may be accepted as subject matter when it seems to violate a supposedly inviolate system of classification. It does not destroy the classification, but it shocks us into attentiveness. The blazing-up of a nova, unmistakably in the sphere of the stars, stirred the interest of men who had assumed the absolute changelessness of that sphere. Aside from such rare exceptions, however, the criterion of recurrence-minimal as it is-is one we seek to enforce absolutely.

Common sense rejects data rather lightly. Science does so more reluctantly, and only provisionally, for science is precisely the quest for "sameness" where common sense despairs of finding it. Born writes that the reality of the natural philosopher or scientist

... presupposes that our sense impressions are not a permanent hallucination, but the indications of, or signals from, an external world which exists independently of us. Although these signals change and move in a most bewildering way, we are aware of objects with invariant properties. The set of these invariants of our sense impressions is the physical reality which our mind constructs in a perfectly unconscious way. This chair here looks different with each movement of my head, each twinkle of my eye, yet I perceive it as the same chair. Science is nothing else than the endeavor to construct these invariants where they are not obvious.

Weizsäcker strikes a harmonic of the same note when he writes:

Certainly experimental science would be impossible if nothing could be perceived, but it would be unnecessary if everything could be perceived.

Now at last we can see the full scope of the criterion for selection of subject matter that arises from our acceptance of the principle of continuity. The unique is rejected, as intractable; the identical is also rejected, as uninteresting. My writing table was here last week, is here today, and I assume I will find it here tomorrow. I can scarcely call this a prediction: identity is the norm I take for granted. And as a norm identity is without interest. For the Greeks "phenomena" were not simply appearances but appearances of change; for us, too, phenomena are divergences from the perfect continuity of identity. Whether we work in science or in common sense, what 
we accept as data will always be precisely those deviations from perfect continuity we find striking enough to excite our interest but not extreme enough to discourage our endeavor to find continuity, even identity.

\section{CONCURRENCE}

I have spoken of the concurrence of the reports of different observers of the same event or thing. Plainly I assume-we all assume-that different observers will see and report the same thing. To any "thing" not so reported or reportable we are quite bold enough to deny status as "fact." Whether as men of common sense or as scientists, we are prepared to deal only with those parts of our experience in which we find a consensus of agreement with our fellows: overt experience, public experience. "Objective facts?" Again no-they depend on human sensitivity and human judgment. But-independent of the sensitivity of any one human-they are the impersonal facts best suited to be subject matter of endeavors seeking knowledge of the "real world" common to all. This much was appreciated very early. Schrödinger quotes the "dark" Heraclitus of Ephesus to the effect:

It is therefore necessary to follow the common. But while reason is common, the majority live as though they had a private insight of their own.

Those who speak with a sound mind must hold fast to what is common to all, ... .

The waking have one common world, but the sleeping turn aside each into a world of his own.

Taking this "in the epistemological sense," Schrödinger himself writes:

Heraclitus is well aware that, actually, there is no difference between the sense perceptions in dreams and in waking. The only criterion of reality is being common to all. This is the basis upon which we construct a real world around us. All spheres of consciousness partially overlap-not quite literally, that is impossible, but by means of physical reactions and communications, which we have learned to understand in each other. The overlapping part of the spheres of consciousness forms the world that is common to all.

However clear in principle, the criterion of concurrence involves serious uncertainties in application. For certification of an observa- 
tion as "fact" we claim to require a general concurrence of "normally constituted observers." How are we to recognize those normal observers? Beyond the obviously enormous variation in mental characteristics of men, the investigations of R. Williams and others demonstrate that even as chemical machines men are never identical with one another. Whatever the situation in the "external world," human experience of it must be variable because the experiencing human reporters differ in biochemical constitution. These differences make themselves felt most notably in reports of tastes and odors. They are less noticeable in reports of visual, auditory, and tactile experience. Even here, however, different individuals falling within the range of "normal" deviations from the average may make significantly different reports. Thus Polanyi reminds us of

. . . the famous case of the Astronomer Royal, Nicholas Maskeleyne, who [in 1796] dismissed his assistant Kinnebrook for persistently recording the passage of stars more than half a second later than he, his superior. Maskeleyne did not realize that an equally watchful observer may register systematically different times by the method employed by him; it was only Bessel's realization of this possibility which 20 years later resolved the discrepancy and belatedly justified Kinnebrook. Experimental psychology, of which Bessel thus laid the foundation, has since taught us universally to expect such individual variations in perceptive faculties.

Normality, then, is a fairly remote abstraction. Were we to insist that "fact" must represent the rigorous agreement of "normal observers" we might find very few facts indeed. How to proceed? To reject a deviant report or reports ex post facto, as due to "abnormality" of the reporters, may seem essentially to vitiate the concurrence criterion. For then it is not the agreement that makes the fact but we who, by forcing the agreement, in part create the fact. Had we means to detect abnormality independent of failure to concur in observation reports, our position would be stronger. For major abnormality such means are usually available-lunatics are often recognizable as such and we may decline to use them as observers. Less striking abnormalities may also be recognized by the exercise of judgment. Proceeding in this way, most potentially deviant reports might be eliminated by rejecting as qualified observers those likely to give such reports.

We can now gain the support of the profound normalizing effect 
of the concepts used by all observers-an effect particularly marked for the tactile and visual data with which both science and common sense prefer to work. Moreover, for many of the purposes of both, perfectly concordant reports are-however attractive abstractlysimply unnecessary. Finally, if we obtain some $99 \%$ of reports concordant within the range of acceptability we are in practice quite content to reject the $1 \%$ of discrepant reports as due to observational error or some hitherto undetected abnormality of the observers. But, even after all these dispensations, still the criterion of concurrence is not reduced to a usable form. For we never could, or do, require a convention of all normal men to attest their concurrence in a "fact." Ordinarily we are satisfied to accept the reports of only a few, and commonly of but one, observer-if the observation involved is one we judge simple and unambiguous. Indeed from a single observer we judge particularly competent we will accept even reports of observations not at all simple and unambiguous. Polanyi tells us that:

\begin{abstract}
... members of the Fifth International Botanical Congress [1931] declared that "the concept of most species must rest on the judgment and experience of the individual taxonomist," . . . Reflecting on this discussion on the definition of a species, S. C. Harland recalled how in Fanny's First Play, by Bernard Shaw, the dramatic critic replies to the question whether the play was a good play, that if the play was by a good author, then it was a good play. "The situation would appear to be somewhat similar," writes Harland, "in regard to what constitutes a species."
\end{abstract}

In principle the criterion of concurrence is enforced absolutely; in practice we leave large scope to individual judgment-which ordinarily assumes concurrence-and there is yet no indication that, in the vast majority of cases, we have erred in doing so. Common sense seems to make generally sound judgments of the "simple and unambiguous" observations in which the report of one "normal" observer is simply repeated when additional witnesses are called in. Science does as well-and even better in that often it can so simplify the observations to be made that the possibility of observational error is minimized. At the extreme of simplification, observation is reduced to reading the position of a needle that moves across a graduated scale until it points to "the result." Judgments of brightness made with the unaided eye are notably variable, and unreliable, as every 
amateur photographer well knows. But the pointer reading of an exposure meter yields an observation in which practically everyone at once concurs. A second major gain: the judgment of "normality" is enormously simplified. However deviant from the norm the observer may be, if he is not obviously lunatic or blind we feel that his report of pointer position can be trusted.

The use of instruments in general, and pointer-reading instruments in particular, enormously facilitates the application in science of the concurrence criterion of "fact" acknowledged by both science and common sense. Such instruments offer us important new powers, but not a general panacea. Instruments may be insufficient even to resolve all problems of concurrence: the Maskeleyne-Kinnebrook episode involved an instrumental observation closely akin to pointer reading. And certainly the major problems of taxonomy seem unlikely to be resolved by any conjectural "reduction" to pointer readings. Finally, instruments cannot ensure "objectivity." "Pointer reading" may offer so unequivocal a subject for observation that it does not overtax the modest endowment of a Cyclopic moron who can distinguish only black and white. But in principle his observation is not one whit more "objective" than an observation in which we deploy our sensory faculties more extensively. With our instruments we more readily attain the "facts" in which all concur. Warranting their impersonality, our concurrence cannot warrant their "objectivity." But have we need for any such warranty? However "objective" or "subjective," all recurrent experience of which we can attain concurrent reports is potential subject matter for science. Whether it becomes actual subject matter depends on a third criterion, to which we now turn.

\section{REGULARITY}

In both science and common sense we work upon experience until it has been reduced to an order on which predictions can be founded. Data which seem to fall beyond any hope of such reduction are apt to go neglected, regardless of their intrinsic interest. To such data we may even deny status as fact-as when Bernard writes:

. . . if a phenomenon, in an experiment, had such a contradictory appearance that it did not necessarily connect itself with determinate causes, then reason should reject the fact as nonscientific. . . . in the presence of such a fact, men of science must never hesitate; they 
must believe in science [which Bernard here equates with determinism] and doubt their means of investigation.

Spawn of our faith in the principle of determinism, the criterion of regularity here comes in play.

Nowhere is the need for judgment more apparent than in use of the criterion of regularity. Polanyi remarks that crystallographic analysis

. . . defines a polyhedron which is taken to be the theoretical shape of a crystal specimen. It embodies only such aspects of the specimen as are deemed regular and in respect to these it is required to fit the facts of experience; but otherwise, however widely the crystal specimen deviates from the theory, this will be [firmly ignored and] put down as a shortcoming of the crystal and not of the theory.

Theoretic judgments are necessarily involved whenever the scientist brings the criterion of regularity to bear and, since his theories or his interpretations of them may err, his judgments may err. Yet always he must apply the criterion of regularity, always he must make a beginning somewhere-with just those data he judges potentially reducible to order.

The detailed course of a feather falling in air is totally unpredictable in the view of common sense and, beyond noticing that the feather falls irregularly, common sense does not concern itself with this matter. The scientist judges that the fall of the feather is completely determined in principle, but complexly determined by multiple conditions difficult to evaluate, much more difficult to control. Such falls are recurrent (though not reproducible); at least approximately concurrent descriptions of them can be obtained from different observers-and wholly unexceptionable descriptions from high-speed stroboscopic photography. But these data seem still so little accessible to reduction to order (and, given our theoretic views, so little likely to teach us anything new) that they are rejected as subject matter.

Consider mirages. Irreproducible in detail, they are yet recurrent. Moreover, we get substantially concurrent reports of them from all those present at a given place at a given time. Beyond noting, perhaps, that mirages are frequent in desert conditions, common sense declines to deal with data on mirages: it sees no prospect whatever of reducing them to determinist order. This lack of orderability, not the possible presence of a "subjective" element, is what makes the 
mirage data unacceptable. That is made perfectly plain by the treatment of such data in science. For at last they do become scientific data-precisely when scientists first conceive the hope of associating these data in a determinist order with others in the general domains of optics and meteorology. This hope being entertained (and since gratified), mirage observations become data every bit as acceptable as subject matter for science as the stick ("subjectively?") seen bent in water, and phenomena of refraction more generally.

What of the more genuinely subjective experiences of dreams, hallucinations, apocalyptic revelations, and so forth? The criterion of concurrence may seem permanently to rule them out of court: nobody else is or can be a witness of my dream. However-though the dream is not certifiable as fact-my report of a dream is so certifiable, by the concurrence of multiple observers of me. Rejecting (as "idle superstition") the ancients' interpretations, modern common sense refused to concern itself with reports of dreams amply recurrent and, as reports, certified by the concurrence of witnesses. Reports of dreams may be related to the wearing of a crown, and reports of hallucinations to excessive use of alcohol, but beyond this common sense seems unwilling to go-unwilling because unable: there seems to be no detectable regularity in the reports of dreams, which come sporadically and without recognizably necessary antecedents. Science shows the same unwillingness as long as it shares the same incapacity. But as soon as we conceive a possibility of ordering these reports of subjective experiences they become acceptable subject matter. Thus details in the reports of dreams, formerly rejected as meaningless, become data of scientific interest to Freud and his followers.

Consider a perfectly parallel case involving "physical" rather than "mental" experience. About 50\% of human observers report the taste of p-ethoxy-phenylthiocarbamide as intensely bitter; the other $50 \%$ as tasteless. My experience of the thiocarbamide is every bit as "real" as my experience of a pointer moving àcross a graduated scale; but there is a fundamental difference in the matter of concurrence. The position of the pointer is certifiable as fact and the taste of the thiocarbamide is not. But the reports of bitterness or tastelessness do meet the criterion of concurrence and are certifiable as fact by the concurrence of multiple observers of the tasters. What will be done about such facts? As long as there is no prospect of involving them in some regular pattern-essentially nothing. But interest flickers into 
life with the discovery that this capacity for tasting is inherited with the statistical regularity of a dominant Mendelian trait; and would blaze up if this capacity could be related to other, independently determinable, data-e.g., the presence or absence or variable constitution of some particular enzyme.

\section{DATA AND JUDGMENT}

Common sense deals primarily with what is experienced by all mankind; science encompasses, in addition, what is experienced, in the laboratory, by but a few. This distinction seems unimportant: the special experience of scientists is potentially available to all willing to enter the laboratory. The fundamental distinction between the data acceptable to science and those acceptable to common sense arises from what has repeatedly been stressed: the involvement of judgment in our use of the three criteria for the selection of subject matter shared by common sense and science.

An "object in the sky" judged to be an alien spaceship is a datum of interest to astronomers; classified as a case of mass hallucination it is, at most, of marginal interest to psychologists. Thus long before our judgments determine how we arrange our data, they determine what we accept as data. Like the principles of jurisprudence, our criteria for the selection of subject matter offer general guide lines but not detailed rulings. And even while the scientist works as detective he must anticipate how, acting later as judge, he will interpret the general criteria in specific rulings on the admissibility and weight of the various possible items of evidence. A priori his wisdom cannot be appraised: a posteriori his judgment passes subject to review. If a scientist finds, in some particular selection of data, hitherto undetected elements of continuity and determinism-we acclaim him a man of genius; failing in his quest, his judgment is by us condemned-we dismiss him as a misguided fool, or a visionary who sought to do what cannot be done.

As it begins science judges the acceptability of subject matter much as does common sense. As science develops-as its view of the world becomes more highly elaborated-it makes these judgments differently. It may then come to pay attention even to data common sense would scorn: a pointer reading is, after all, a rather trivial item of experience to which we pay attention only as, given the theoretic views of science, we grasp its significance. Made bold by successes 
already won, scientists come to seek more widely and tenaciously for continuity and determinism, even where common sense wholly despairs of finding them. Particularly as regards regularity, the scientist's horizon of expectation is ever expanding. He finds then even in wholly impersonal data a compelling excitement to which the mar. of common sense is utterly unawakened. For, says Maxwell:

It is a universal condition of the enjoyable that the mind must believe in the existence of a [discoverable] law and yet have a mystery to move in.

\section{The Organization of Experience}

Science involves a fourth stage in the organization of experience, of a sort unknown to common sense. But this fourth stage is not a superstructure built on the otherwise unchanged foundation afforded by the first three. The colligative relations of science differ from those of common sense, if only because they involve different concepts. And, deploying different concepts, the scientist often arrives at constructs different from those of the man in the street. Let us now subject science to the successive steps of analysis earlier carried out on common sense.

\section{CONSTRUCTS}

The scientist is likely to make common-sense constructs of whatever does not immediately concern him as scientist. Conditioned by a massive common heritage, he is predisposed toward the constructs made by the man of common sense whenever they share the same purpose. Presumably the scientist "sees" the table beneath his apparatus as just the "support" it ordinarily seems to the common man. The situation changes drastically when the scientist turns to things that command his attention as scientist. When he looks at his manometer he does not, like the ordinary man, see a construction of glass, held on steel supports, and containing mercury. Instead he is apt to "see" only one aspect of his manometer, and that so trivial, apparently, it might wholly escape the man of common sense. The scientist sees focally only the difference in the height of the mercury columns. His grasp of the scientific concept of pressure shapes his construct of the manometer to highlight what is, for him, "the only thing that matters." 
Where new concepts are used, new constructs are made. Often these scientific constructs may appear superior approximations to "pure observation reports." Sufficiently taxed (e.g., by optical illusions), even common sense will seek to reform its constructs-to separate what is "given" from what, plausibly but perhaps groundlessly, has been inferred. But history has taught the scientist that a great deal of effort may be invested profitably in this separationin trying to free constructs from their customary freight of unconscious inference. This procedure the scientist calls "paying attention to the observables," and as a procedure it has been of crucial importance to such 20th-century developments as quantum mechanics and relativistic mechanics.

The scientist's resolve to "pay attention to observables" is too often mistaken for a profound new insight of modern science. That resolve is already detectable in the remote beginnings of science with such as Aristarchus and Copernicus. Galileo writes:

... I cannot find any bounds for my admiration how reason was able in Aristarchus and Copernicus to commit such a rape upon their senses, as in despite thereof, to make herself mistress of their credulity.

Of course there was no rape upon the senses: what reason did was simply to undo or redo what reason had formerly done. By reason, using new concepts, Aristarchus and Copernicus managed to recast or reconstruct what had long passed as the naked report of the senses though it contained a large component of "credulity": plausible inference with which it had been laden by common-sense reason.

"The naked report of the senses," shown in Chapter I to fall beyond the reach of common sense, is for science, too, an utterly unattainable ideal. Also, I think, in some degree a sterile ideal. If I report pointer readings and nothing more I report nothing of interest to science. Interest develops precisely as the pointer readings are associated with inferences. Consider Poincarés example:

I observe the deviation of a galvanometer by the aid of a movable mirror which projects a luminous image or spot on a divided scale. The crude fact is this: I see the spot displace itself on the scale, and the scientific fact is this: a current passes in the circuit.

if I ask an ignorant visitor: Is the current passing? he looks at the wire to try to see something pass; but if I put the same question 
to my assistant who understands my language, he will know I mean: Does the spot move? and he will look at the scale.

Observe the perfect parallelism of this and a common-sense situation sketched by Herschel:

In Captain Head's amusing and vivid description of his journey across the Pampas of South America occurs an anecdote quite in point. His guide one day suddenly stopped him, and, pointing high into the air, cried out, "A lion!" Surprised at such an exclamation, accompanied with such an act, he turned up his eyes, and with difficulty perceived, at an immeasurable height, a flight of condors soaring in circles in a particular spot. Beneath that spot, far out of sight of himself or guide, lay the carcass of a horse, and over that carcass stood (as the guide well knew) the lion, whom the condors were eyeing with envy from their airy height. The signal of the birds was to him what the sight of the lion alone could have been to the traveler, a full assurance of its existence.

The scientist's constructs carry no small burden of inference: seeing a spot of light, he "observes" (and reports) an electric current. The scientific constructs differ from those made by common sense in the nature of their inferential freight, perhaps also in the reduced magnitude of their freight of unconscious inference. But of this there is always some. Braithwaite conceives that:

The peaks of [theoretic] science may appear to be floating in the clouds, but their foundations are in the hard facts of experience.

Popper proposes a related metaphor which, however, finds "softness" in Braithwaite's foundations:

The empirical basis of objective science has thus nothing "absolute" about it. Science does not rest upon rock-bottom. The bold structure of its theories rises, as it were, above a swamp. It is like a building erected on piles. The piles are driven down from above into the swamp, but not down to any natural or "given" base; and when we cease our attempts to drive our piles into a deeper layer, it is not because we have reached firm ground. We simply stop when we are satisfied that they are firm enough to carry the structure, at least for the time being.

The towering structure of theoretic science is then stable only as, from time to time, scientists of the calibre of Aristarchus or Coperni- 
cus or Einstein recognize a need, and an opportunity, to drive new and deeper pilings-to reformulate the constructs. That opportunity presents itself as new scientific concepts are brought into play.

\section{SCIENTIFIC CONCEPTS}

Scientific concepts, like their counterparts in common sense, fall into two distinguishable though not wholly distinct groups. There are, first, the lower-level indicative concepts, having reasonably clear experiential denotations, which give us power to create and use the colligative relations through which we seek to forecast the future. There are, second, the more abstract explicative concepts, often lacking such denotations, which give us power to work a further organization of experience by bringing the separate colligative relations into association with each other.

Extended discussion of the second group of scientific concepts I defer to Chapter VIII, where I consider them in the context of the theories they make possible. Serving different (logical) functions, they differ radically from their common-sense counterparts; serving a similar explicative role, they somewhat resemble those counterparts. Thus, for example, we forever seek to construe what seems strange and puzzling in terms of analogies with what we have come to find familiar and take for granted. In common sense we may conceive physical causes on the analogy of the emotions we suppose to be the "cause" of human activity. In science purpose or volition is not acceptable as a physical cause; but the concept of "force," which loomed so large in Newtonian mechanics, is an anthropomorphic cause patterned on a familiar analogue. Meyerson correctly draws the general conclusion that

. . starting from a conception of the world such as our naïve perception offers, the physicist has never transformed it save by putting into play the very rules according to which this conception was constituted. He has continually substituted the invisible for the visible, but what he has created is of the same order as what he has destroyed.

The strangeness of scientific concepts. The concepts of science, whether indicative or explicative, often strike us as "strange." They must seem strange. The common-sense concepts, grasped in childhood, we find sanctified in the very structure of everyday language. The scientific concepts we acquire much later in life; only with diffi- 
culty can we come to feel at ease with these late acquisitions, and with the exotic language in which they are expressed. We may then be led to compare scientific concepts unfavorably with the "natural" concepts of common sense. This is nonsense, for, as Einstein observes:

Physical concepts are free creations of the human mind, and are not, however it may seem, uniquely determined by the external world.

When the task is different, the most appropriate conceptual tool may well be different. Thus, for example, if through the indicative concepts of common sense we arrive at predictively powerful colligative relations, those concepts fully discharge the function for which they were devised. But these relations of common sense do not lend themselves to the fourth stage of organization sought in science. And so in science we seek, through indicative concepts expressly designed with this different function in mind, for relations that do so lend themselves. For conceptual evolution common sense and science thus constitute two distinct though overlapping domains, imposing distinct though related demands. The everyday concepts, enormously successful in the domain of common sense, do not thereby establish any claim to fitness for survival in the domain of science. Because the motions of swimming are useful in propelling us through water, it does not follow that we do best to breast-stroke along the beach.

Devices of men bent on the quest for a comprehensive rational order in experience, the concepts of science fall subject to natural selection in the world of man's experience. How by "natural selection"? The scientific concept "cell" is a thing-concept that synthesizes, from various perceptual elements, a "something." Like the commonsense concept of "physical object," "cell" refers also to a conspicuously abstract something: an extraordinary variety of actual forms are all grouped under the rubric of one diagrammatic representation of a typical cell. In both cases the concrete value of the abstract concept is abundantly demonstrated, however, as we find that what it joins together can, in thought or in action, be handled effectively as a something. Passing on to class-concepts, we encounter here the traditional tools of classification so richly represented in the endeavors of biologists and geologists. A less hackneyed example, perhaps, is the chemist's concept-"carbon"-which holds fundamentally identical substances as different as charcoal, graphite, and diamond. Such 
a concept cannot but seem bizarre: it ignores enormous ("superficial”) differences, and takes as its basis of classification qualities far less obvious than those on which common sense founds its classifications. However bizarre, these concepts of science nevertheless satisfy a final and decisive criterion: with them we find major elements of order that the classificatory concepts of common sense fail to reveal. Says Bronowski:

It is not obviously silly to classify flowers by their colors; after all, the bluer flowers do tend to be associated with colder climates and greater heights. There is nothing wrong with the system in advance. It simply does not work as conveniently and as instructively as Linnaeus's classification by family likenesses.

Thus, Mill correctly observes, general classificatory conceptions formed in advance of complete knowledge must always correspond to Bacon's "notiones temerè à rebus abstractae":

Yet such premature conceptions we must be continually making up, in our progress to something better. . . . That the conception we have obtained is the one we want, can only be known when we have done the work for the sake of which we wanted it; . . .

Concepts and the anatomization of experience. Always the search for order is guided by the scientist's feeling for the nature of what Bronowski calls "the reality behind the appearances." This feeling finds clearest expression in his explicative concepts-the "causes" he thinks significant-but these profoundly influence his construction and use of indicative concepts. An immense advance in science may thus have its roots in an alteration of point of view which produces a new or newly modified group of indicative concepts. Inspired by the Pythagorean faith that mathematics is the language of the book of Nature, Galileo brought a new "set" to a realm of experience that had defied all earlier efforts at conquest. Studying moving bodies, Galileo proposed to ignore the weight, size, texture, color, and everything else that makes each one a distinctive body, and to consider instead only the mathematically describable motion of which all partake.

"Velocity," “acceleration," and some of Galileo's other key concepts are typical quality-concepts, indicating one aspect with respect to which many otherwise disparate objects may be compared, and so related, with one another. However, the "qualities" with which 
Galileo worked do not merely relate objects but, in a sense, create order. Consider that, in his experiments, Galileo could not observe "velocity"; he could observe only that a moving body occupies various spatial positions at various times. The concept of velocity suggests a particular co-ordination of these observations. We divide "distances traveled" by "elapsed times." The quotients so extracted from the actual observations are what we call "velocities." The virtue of such indirection becomes manifest if, for example, we encounter a case of uniform motion: underlying all the changes of position with time we find an unchanging "velocity," in the apparent chaos of kinematic experience we find an element of identity.

Suppose we find no such case of uniform motion. We may then seek through still more abstract concepts for the elusive element of identity we hope and expect to find underlying all change. We can turn to the subtle concept of "acceleration." Accelerations we may extract from the elapsed times and the previously calculated velocities. And in cases of free fall Galileo's intuition finds its triumph. Underlying the changing times and positions of falling bodies generally, there is an unchanging acceleration. Thus we have found not merely order but a genuine element of identity where common sense finds none. Common sense fails here because-no prolific constructor of new concepts-it is most unlikely to create quality-concepts referring to no particular observables but to special, superficially mysterious combinations thereof. Scientific quality-concepts involving such combinations are of course in no way peculiar to kinematics. Thus the concept density teaches us to see-underlying all our experiences of weight and volume with a given substance-a "something constant": the quotients of weights divided by volumes. Such discoveries, investing us with new predictive powers, we express in colligative relations.

\section{COLLIGATIVE RELATIONS ("LAWS") -}

The relations we can find and express depend on the indicative concepts we devise and deploy. Science here enjoys a superiority qualitative as well as quantitative. The abundant stock of scientific concepts makes possible specialization of function; precision of denotation need not be so deeply compromised by the search for general applicability. In science we thus arrive at colligative relations apparently so far superior to those of common sense we regard them as 
"laws of nature." These laws are still, however, humanly created and expressed laws, not unmarked by the characteristics of their creators. By no stretch of imagination can such laws be considered mere "economic descriptions" of observables, or the like. At first sight we may conceive the possibility of easily arriving at, say, the law of the lever, purely on the basis of observation. Imagine how we might try to do so-assuming ourselves untutored savages who, however preposterously, have a modern outlook, a taste for systematic study, and some knowledge of elementary mathematics.

The law of the lever. Our casual experience of moving boulders with a wooden beam is an inadequate foundation for discovery of the law of the lever. In the opening scene of this little fantasy we are found sitting in front of our cave, manipulating an assembly of a light, rigid, straight bar (if not a titanium rod, perhaps a fragment of a giraffe's shinbone), a sharp horizontal support (if not an agate knife-edge, then a suitably chipped rock outcropping), and an assortment of objects. Do not ask now why we should concern ourselves with this curious array of things in the first place: we are too busy discovering the law of the lever.

We hang two objects, arbitrarily chosen, at arbitrarily chosen positions along the bar. Sliding the bar along the support, $F$, we discover one curiously unique position between the points of suspension of the two objects. When this position falls directly over $F$, the bar remains at rest in a horizontal plane-after a series of oscillations we agree to ignore as "irrelevant." When this state is attained we say: "The system is in balance." Without making any other change, we now add a third object to one of those already suspended. We find that the system then is no longer in balance. But balance can be restored, we find, by shifting the bar along the support $F$ in the direction that approaches the added object to $F$. Continuing such trials, we find that, by suitable placement on the support $F$, we can bring the system into balance even when we have but one object on one side of $F$ and a great many objects, suspended together, on the other side.

Already we have passed far beyond "pure observation." Into our report have crept distinctly conceptual elements like side, support, one and many, horizontal, system, etc. Moreover, if we are still reasonably close to pure observations, we are also painfully far from having gotten anywhere with them. Clearly we are not going to get 
anywhere until we structure the percepts with some further concepts. Though the lever's law be a "law of nature," yet we must contrive to grasp it-contrive with tools mental as well as physical. Though simple, the physical equipment is of considerable artificiality; the concepts we need, though also simple, are substantial abstractions.

The first of these concepts is "distance." Speaking of the "distance" between $F$ and the point of suspension of an object, we focus attention on what is, for our purposes, the best characterization of the separation of $F$ and object. "Distance" we hold to be independent of the material composition and surface characteristics of the bar along which it is measured. This kind of independence is not a priori obvious: if concerned with the distance of a place to which we wish to journey, we do consider the nature of the intervening terrain (swamps, mountains, rivers, etc.). Indeed, this kind of distance may be more meaningfully expressed in the Indian "moons" (a primitive unit of space-time?) than in miles or kilometers. But let us suppose ourselves fortunate enough to conceive "distance" as a "length" measured along the beam with a meter stick (or a straight spear graduated in arbitrary but equal intervals, and carefully preserved under goatskin in an atmosphere of constant humidity within a vault at the back of our cave).

"Distance" singles out one aspect of a complex experience; the concept "weight" demands an abstraction at least as difficult. Saying "weighty objects" we come to consider one particular aspect of our experience of objects that may be simple or complex, fluid or solid; completely different in geometric form, material composition, and so on. We so initiate a dissociation of the physical object: from "moving bodies" Galileo passes on to "motion"; from "weighty objects" we pass on to "weight." The "weight" of a body we may conceive as corresponding to the muscle pull we exert to lift it. But this correspondence is not uncomplicated: we must, for instance, discriminate the effort of lifting from the mere awkwardness of lifting a body of unfelicitous bulk or shape. (Was the difficulty of this discrimination responsible for what was once the accepted opinion-that a corpse weighs more than the pliant living body it was?) Fortunately our appreciation of weightiness can be sharpened as we recognize one obvious special case of the law of the lever. Suspending objects at equal distances from the support $F$, suppose we choose two objects that bring the system into balance when so suspended. The two ob- 
jects so selected-dissimilar perhaps as a sack of millet and a bar of gold-have a remarkable relation. Interchanging their positions, we find the system still in balance-an unprecedented development. More remarkable still, we find that in all situations of our lever system anything balanced by the millet is alike balanced by the gold. The equal-arm balance thus singles out that quality-characteristic of millet as of gold-we agree to call "weight." We now adopt a certain object (e.g., a stone) as an arbitrary unit of "weight," just as we took a spearshaft as our unit of length. Another object that balances this stone on our equal-arm balance is then also of one-unit weight. An object balanced by two one-unit objects is said to have two units of weight, and so on.

Grasping the concepts weight and distance, we can at last state our problem in an abstract sketch of the system:

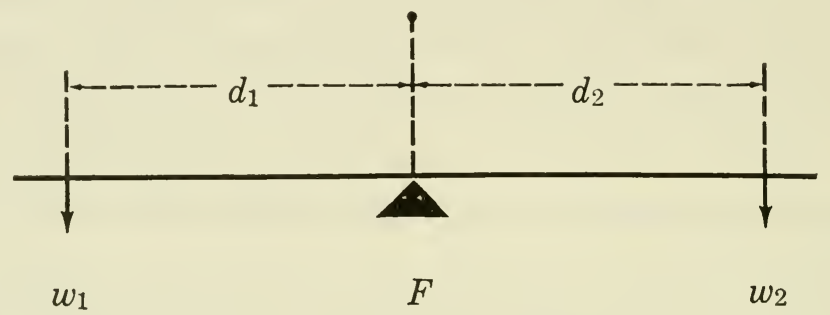

Placing various weights at various distances along the shaft, we now expect soon to arrive at the law that governs the equilibrium of the system, namely:

$$
\frac{w_{1}}{w_{2}}=\frac{d_{2}}{d_{1}}
$$

This "discovery" of a "purely phenomenologic" relation is no drama of man's intellectual powers, but a fantasy. Why? We assumed ourselves untutored savages. The law was first enunciated by no untutored savage. Much is, then, hidden in the qualification "untutored savages with a modern outlook." We began with the avowed intention of discovering the law of the lever, which we already knew, but not even an untutored savage with a modern outlook would have such knowledge. At the very least we assumed awareness of a problem and the desire to solve it, though this problem does not force itself on us in an experience rich in oddments of many more pressing varieties. Even in formulating our problem we have assumed 
grasp of the concepts of weight and distance, which are not "given" in experience, and we have further supposed their denotations at once established with contrivance of auxiliary experimental tools like the meter stick and the equal-arm balance. A further element of fantasy develops around just such tools. In a real inquiry, directed toward the discovery of a relation not already known, the clean uncomplicated beam and fulcrum with which we began would appear not at the beginning but very near the end. We specified as the beam a light, straight, rigid body-e.g., a segment of a giraffe's shinbone. But this prescription is not "given" us. A priori we might as well have picked the jawbone of an ass, a subject much less suitable for the discovery of the law of the lever. Starting with a simple device, and concepts, suitable for the solution of a problem already clearly formulated, we began with more than half the battle already won.

Perhaps the greatest element of fantasy in my sketch has yet to be noted. I assumed measurements that lead to the law of the lever. These were synthetic measurements; actual measurements do not yield the simple equality of weight and distance ratios indicated in the law. The weights are, after all, not the only weighty objects in the system: the beam itself has weight. Neglecting this we neglect an essential element of the situation. What to do? We cannot simply introduce the highly sophisticated concept of a "center of gravity"; this surely will not have occurred to us before we have even grasped the law of the lever. Perhaps we might think to stipulate use of a very light beam and very heavy weights. But then a different complication sets in when the beam begins to bend.

Ultimately there are three major courses open to us. First: we might complicate our statement of the law of the lever to allow for the weight of the beam, its rigidity, the lengths by which it projects on either side of the fulcrum, etc. Such an expedient is repugnant to science and to common sense alike; we arrive then at an awkwardly complex expression difficult to think about and difficult to use as a predictive device. Second: we might say our results are "good enough" to justify statement of the uncomplicated law of the lever as a rough working rule. This would be the course followed in common sense. Third: we might adopt a course characteristically that of science. We postulate an ideal lever: a straight, rigid, weightless beam-essentially equivalent to a Euclidean straight line-borne frictionlessly on an ideal fulcrum. Feeling we understand why our actual 
results are not perfectly represented by the ideally simple law, we conceive an ideal lever to which, we say, the law would perfectly apply. The practical situation remains wholly unaltered: the intellectual thrust is wholly different. The man of common sense matches law and observation by holding that the ideal law of the lever is only approximate-only a rough but useful description of actual observations. The scientist matches law and observation by holding that the law of the ideal lever is rigorous-adding only that actual observations with real levers are only more or less imperfect approximations to what we would observe were there in the real world ideal levers with which to work. This was essentially the procedure adopted by Archimedes. It represents an immense abstraction.

Denotation. For all its abstractness, the law of the lever remains a colligative relation: its statement involves only concepts with reasonably clear denotations. In "weight" we may seem to deal with an adjectival quality-concept, "weighty," dangerously and absurdly masqueraded as a noun-a "real quality." And "distance" seems an abstraction equally extreme. But unlike the philosophic "qualities," our concepts do have denotations. "Weight" is something crudely indicated by muscular effort and, more precisely, by measurement with an equal-arm balance; "distance" is something given crudely by visual inspection and, more precisely, by measurement with a meter stick.

The instruments somewhat sharpen and clarify the conceptual denotations, but there remain major problems they do not even begin to solve. This is nowhere clearer than in the matter of our concept of "lever." The ideal lever, to which alone the ideal law applies rigorously, exists only in our minds. We might embark on elaborate specifications of certain particular "levers" we have found to be adequate approximations to the ideal. Yet we do not; only by foregoing such specifications do we first gain power to make predictions about systems substantially different from those with which we have already worked. Having in mind the ideal lever-knowing what we're looking for-we can discern lever action in systems in which the presence of any lever is far from evident. We come thereby to make excellent predictions about the performance of such devices as wheel (or crank) and axle, compound pulleys, and the like. As always, such generality is purchased only at cost to reliability. Experience alone 
can now teach us to be wary in applying the simple law to systems highly "non-ideal": e.g., with "beams" made of a roll of paper or a stick of chewing gum. We learn that if we have a reasonably light, reasonably rigid, reasonably straight beam with reasonably good bearing surfaces and reasonably heavy weights-then we can make reasonably accurate predictions of its behavior. Most important of all, we must learn to judge what is meant by "reasonably." Just as in common sense, then, denotation remains in science something of an art, the proper practice of which we learn only by experience.

The ensemble of colligative relations. Compare the colligative relations of science with those of common sense. The "laws" of science are far more subtle, far more consistent with one another, far more sophisticated. We may suppose them also far superior in reliability and generality but-in view of the tension between these two factors -the superiority cannot be absolute. Given the reliability we expect of it, a "law of nature" must be so formulated that it is almost completely inapplicable in the tangled circumstances of everyday life. Here the rough-and-ready common-sense maxim-however less reliable-is far more generally applicable. Outside his laboratory even the most doctrinaire scientist bases the vast majority of his decisions on common-sense maxim ("horse sense") rather than on scientific law. On the other hand, given the generality we expect of a "law of nature" we must, as we have seen, accept a looseness in the denotation of its conceptual terms resolved only by the exercise of an art. That art being ever necessary-and necessarily ever uncertain-even the "law of nature" must then reduce to the general form of the colligative relation: If $A$, then probably approximately $B$. If a "lever" is so loaded that $w_{1} d_{1}=w_{2} d_{2}$, then probably it will be in balance; or, if we wish a "lever" to balance, then the second weight, $w_{2}$, must be attached at a distance from the fulcrum approximately equal to $w_{1} d_{1} / w_{2}$. Considering what we demand of a scientific law, considering too the range within which we check its predictions and the precision with which these checks are made, the qualifications "... probably ... approximately ..." are needed here as urgently as when we write a maxim of common sense.

I conceive that the crucial difference between the colligative relations of science and those of common sense is irreducible to differences in generality or reliability-or subtlety or consistency or 
sophistication-important though all of these may be. The crucial difference is, I think, simply this: beyond investing us with predictive powers, the scientific relation lends itself to that fourth stage of organization sought by science but inaccessible, and quite unknown, to common sense.

With Poincaré, let us imagine our science systematically built up from relations, as a house is with stones. Common sense provides us with a heap of stones; natural history with heaps of stones carefully assorted according to size, shape, texture, etc. But only in the edifice of science do we have a coherent structure in which the individual stones are bound together in an orderly fashion by the mortar of logic and mathematics. We hope to learn to know the stone by recognizing its position in the house, to master the individual relation by seeing it in the larger context of a postulational system in which it appears as logically derivative. So hoping, we demand that scientific laws lend themselves to such seeing, demand that they constitute subjects suited to the thoughts of men seeking a thorough-going rationalization of experience. The superficially absurd abstractions like the ideal lever here come fully into their own.

We think of science as based on our experience of the world, and so it is. Yet sometimes we seem to ignore that experience, even to deny it. Rather than pondering our real experience of real lever systems we set ourselves to contemplate fictions-an ideal lever and the ideal law thereof. In so doing we make an immense gain. The raw phenomena are complicated and variable; the ideal law, which only sketches them, offers an ideally simple statement about "ideal" phenomena. Considering the colligative relation, we can forget for the time being all the "imperfections" reflected in our actual experience -confused by the only partially determinate effects of a multitude of "secondary factors"-and take as subject a "pure" relation. We begin the difficult task of theoretic construction with ideally simple entities and relations-with readily manipulable fictions represented in terse, abstract, often symbolic form. Such entities are the partless points and widthless lines of geometry, the mass-points (and ideal lever) of mechanics, the ideal gas of pneumatics, the ideal solutions of chemistry-all of them represented by ideal laws. Setting out from these, we may be able to arrive at a conception of some very general postulates from which "follow" a multitude of colligative relations. 


\section{SCIENTIFIC THEORIES}

Rejoicing in possession of myriad predictively powerful colligative relations, we find them less than our heart's desire in two important respects. First is the practical problem of remembering-remembering not merely relations but also, for each, the auxiliary material without which the relation has little value: the "stops" defining its general range of applicability, the "feel" for the reliability of its predictions in various parts of that range, the grasp of the many alternate denotations attaching to its conceptual terms. This first problem arises presumably from the inadequacy of human memory; a second problem arises surely from the intransigence of human aspiration. We want to grasp the origin of scientific laws. We want to know how or why they are as good as they are, and how or why they are defective as they are. Whatever the predictive capacities they give us, these relations leave us unsatisfied until we can "explain" them. There is then the theoretical problem of understanding. Were we able to solve this second problem, we would take a long step toward solution of the first. We easily remember a rational statement involving some hundred-odd words; we find it far more difficult to memorize a hundred-odd words in a nonsense sequence. We seek then to see scientific laws as parts of a higher rational order.

Were our minds and our intellectual heritage different, we might find quite different orders rational. Being what we are, we find supremely rational the order of a postulational system, that particular order of which Euclidean geometry presents us with a familiar example. Such a system stipulates a small number of axioms, and certain rules for handling them. From the axioms, following the rules, we then arrive deductively at a vast number of theorems which are for us thereby "explained." Even today only a few physical theories achieve some distant approximation to the strict form of a postulational system; and this form is still anything but apparent in the representative theories of, say, geology and biology. But scientific theories have invariably the character of postulational systems: always they offer rational correlations in which, from a given set of premises, we deduce an array of theorems we identify with colligative relations. The "deduction" and, even more, the "identification" involve complexities to be examined in Chapter VIII. For the present 
I seek only, through a simple example, to display the three functional roles of scientific theories.

The kinetic theory. With a constant quantity of gas at constant temperature the product of pressure and volume is constant. Through the reasonably clear denotations of its conceptual terms, Boyle's law furnishes a good account of one aspect of our experience of gases. But this account is not perfect: between the law and our experience of gases we find appreciable discrepancies. With these we may deal in characteristic fashion-postulating an "ideal gas" to which Boyle's law is to apply rigorously. Actual gases being then only more or less imperfect approximations to the hypothetical ideal, we apply Boyle's law successfully only as we come to associate with it certain limits in range and reliability. Charles', Leduc's, Dalton's, Gay-Lussac's, and Graham's laws are similar to Boyle's in all essential respects. Each, by a skillful choice of concepts, condenses into a terse abstract statement an immense descriptive and predictive capacity. Each is to a degree both general and reliable, but each carries limits in both range and reliability, and applies rigorously only to an "ideal" case.

Consider now how a rational order is imposed on this entire group of "gas laws." We begin by making some hypothesis about the nature of gases. Here, as in many other cases, we posit the existence of unobservables, thereby to reduce to order the observables described by the colligative relations. Creating the kinetic theory, we postulate that a gas is a space thinly populated with minute corpuscles having a kinetic energy proportional to the absolute temperature of the gas. These particles we suppose to move in accordance with Newton's laws of motion, and to be perfectly elastic in their collisions with each other and with the walls of the container. We have now a model of a gas-but a model still somewhat hard to handle. Hence we make two auxiliary assumptions-inessential to a kinetic theory as such-merely to simplify otherwise difficult deductive operations. IVe postulate that the corpuscles are point-masses of zero volume; and we postulate that, save in their collisions, no forces whatever are active between them. These assumptions cannot be perfectly sound for any gas we handle in the laboratory-since all such gases condense, at temperatures appreciably above absolute zero, to liquids that have appreciable volumes. With the introduction of the two simplifying assumptions, then, we are no longer speaking of actual gases but of a more tractable ideal gas. 
Setting out from our postulates, we now arrive fairly straightforwardly at deduced theorems we delightedly identify as precisely the relations named for Boyle, Charles, etc. This is an immense correlative achievement. Also an immense explanatory achievement: we see how the behavior described by the colligative relations may be produced. Yet our satisfaction is not wholly unbounded. Using a model of an ideal gas we arrive at Boyle's law, which applies rigorously to that ideal gas but not to actual gases. Feeling we understand how the law arises, we are now all the more anxious to understand why it is not rigorously applicable to actual gases.

Ordinarily quite reliable, predictions drawn from Boyle's law are apt to be wide of the mark with gases at high pressures and/or low temperatures. For such failures the relation itself offers no rationale: Boyle's law simply fails, by a larger or smaller margin, under conditions we must then memorize. But with the kinetic theory in hand we can understand these failures: we have only to grasp how actual gases may differ from our hypothetical ideal gas. Our gas model involved two assumptions that cannot be perfectly sound. Boyle's law fails under precisely those conditions in which the assumptions would be least satisfactory. There is failure (1) when the gas pressure is high and the gas volume small, so that the fraction of the total volume "filled" by the corpuscles becomes significant; and (2) when the gas temperature is low, so that the kinetic energy of the corpuscles no longer wholly overrides intercorpuscular attractions. In the shortcomings of the assumptions used in its theoretical derivation, we see the origin of the shortcomings of the empirical law. No longer need we simply memorize the conditions in which Boyle's law fails badly; we now understand in advance that under such conditions it must fail.

A more sophisticated theoretical construction does without the two simplifying assumptions, and yields a new (van der Waals) relation:

$$
\left(p+\frac{a}{V^{2}}\right)(V-b)=\text { constant. From measurable characteristics of }
$$

any gas at its critical point, we calculate a correction term $(b)$ representing the volume "filled" by the corpuscles themselves, and another term $(a)$ representing the intercorpuscular attractions. When the volume of the gas $(V)$ is large-i.e., at low pressure $(p)$ and/or high temperature-the correction terms involving $a$ and $b$ are negligible, and the van der Waals relation then simply reduces to Boyle's 
law: $p V=$ constant. Boyle's law here emerges as a limiting law. However, when the volume of the gas is small-i.e., at low temperature and/or high pressure-the correction terms become significant, and the predictions of the van der Waals relation then differ substantially from the comparatively inferior predictions furnished in these conditions by Boyle's law.

Remembering, understanding, discovering. Our first theory suggests how Boyle's law may arise, and further suggests how it may come to fail where it does fail. Our second theory offers us means to estimate in advance the magnitude of the failure. And Boyle's law here is paradigm of all the familiar gas laws. We can then feel we understand both the successes and the failures of all these laws. The kinetic theory thus proves its effectiveness as an explanatory device.

Always we remember better what we feel we understand, and in any case the need for remembering is now triply reduced. First, we can now derive any of the many gas laws, as it is needed, from a few easily remembered theoretic axioms. Even making no derivation, each law now reminds us of others in the group with which we have come to associate it. Second, making the derivations, we find the simple laws obtainable only with the aid of certain auxiliary assumptions and approximations. Grasping the circumstances in which these are likely to fail, we simultaneously grasp the limits in range and reliability attaching to the simple laws. Third, the task of memory is reduced even as concerns the many alternate denotations attaching to the concepts that figure in the derivative relations. We must still learn some primary denotations, but the "equivalence" of any alternate denotation is itself a colligative relation we come to grasp easily as it too finds theoretic accommodation (ordinarily elsewhere than in the kinetic theory). Scientific theories thus function superbly as correlative devices.

Good theories are more than correlative devices, more even than explanatory devices. Good theories are also heuristic devices-tools that assist us in winning new knowledge. From theoretic postulates we arrive deductively at theorems we identify with known colligative relations; sometimes also at other theorems we identify with colligative relations not previously known. We are then led to look for certain things not before seen, or perhaps even thought of. Thus, for example, the kinetic theory suggested to Maxwell the novel idea that gas viscosity should be independent of gas pressure. Investigat- 
ing, we find this deduction easily confirmable, as are indeed a good many others of its kind drawn from the kinetic theory.

Theories and nets. In the first stage of the organization of experience flocks (in the sense of locks of wool or hair) of hypothetical "atomic stimuli" are formed into immediate, individual constructse.g., "objects" that exist stably in an otherwise chaotic welter of stimuli. In the second stage classes of objects and of the qualities of objects are developed. Concepts are formed; from selected groups of fibers are spun multiple fine threads which, in the third stage, are intertwined to form colligative relations. At this level the maxims of common sense, the rules of the craftsman, and many of the laws of the scientist appear together. Only in science do we push on further, to a fourth stage, in a fully self-conscious way. We weave the relations together in postulational systems, theories. Says Popper:

Theories are nets cast to catch what we call "the world": to rationalize, to explain, and to master it. We endeavor to make the mesh ever finer and finer.

He who realizes the existence of such a conceptual fabric, and is capable of lifting it, carries with it all its cords, all the colligative relations it accommodates.

This metaphor grossly oversimplifies: each "stage" is not simply produced from, but also helps to produce, those that precede it. Moreover, in describing the functions of scientific theories, the case I have sketched is too "ideal." Qualitatively the elements of gain are correctly represented. Quantitatively the gains may be less impressive, because fully to master a scientific theory is a task far more difficult than I have indicated. For example, from the theoretical postulates the manifold colligative relations descend by deductive chains that normally involve, beyond conscious assumptions and approximations we have to learn to use as appropriate, further assumptions and approximations the experienced scientist makes automatically, without full awareness of what he is doing. To master the theory, and so to command the relations it holds in chains, one then requires-beyond premises and rules for their manipulation-a "feel" for the system. Immensely powerful tools, scientific theories are also imperfect tools, handled with full effectiveness only by those who have that subtle art of which Toulmin writes: 
It is part of the art of the sciences, which has to be picked up in the course of the scientist's training, to recognize the situations in which any particular theory or principle can be appealed to, and when it will cease to hold.

Nobody today can master all of science because nobody today lives long enough to master the arts of all scientific theories. But he who has mastered a few major theories does thereby come most literally to comprehend certain entire domains of his experience. 


\section{CHAPTER III}

\section{The Anatomy of Science}

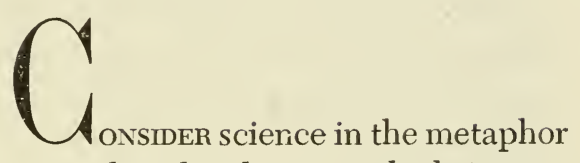

of social organism, swimming in a cultural milieu on which it acts, and by which it is acted upon. What that organism is today, it has become by evolution. Beginning with a brief historical survey, we become aware of certain features of science, today so taken for granted that we recognize their nature and importance only as we look back upon the antique science in which they were absent or had at most a rudimentary development.

\section{The Evolution of Science}

Along the continuum that stretches from common sense to science, only a somewhat arbitrary act suffices to define a "beginning." Arbitrariness is minimized, however, if we take as criterion of the emergence of science the appearance of its characteristic fourth-stage organization. Historically this appearance has something of the abruptness of a mutation.

Neolithic man had already mastered agriculture and animal husbandry; milling, baking, and brewing; tool-making; pottery- and brick-making; etc. Somewhat later the great delta civilizations mastered the complex manufacture of bronze, which required a combination of ores mined in places hundreds if not thousands of miles apart. Still later, men mastered the even more complex procedures involved in successful smelting of iron. To carry on any of these processes is to have mastered whole sets of predictively powerful colligative rela- 
tions of the type: If you take ore of this (describable) sort and treat it in this (describable) manner, then probably you will obtain a metal having approximately these (describable) properties. Such relations go far beyond the content of ordinary common sense, but I cannot base on them a claim that early man was already a scientist. I suppose that observation, unsystematic empiricism, and commonsense reasoning provide an ample foundation for the discovery and use of such relations-the sets of which are the maxims, recipes, formulas, and rules that constitute craft traditions.

No craft tradition is properly denominated science. If we deny to carpentry status as "science" we cannot so dignify the colligative relations developed by early man-immense achievements though they were. This position I maintain even when some of the relations are semi-quantitative, or even fully quantitative. The relations of practical metallurgy must already be at least semi-quantitative. The Babylonians go on to develop fully quantitative formulas for prediction of eclipses; the Egyptians, some of the theorems of physical geometry. To such relations one is reluctant to deny status as science, but one is hard pressed to see how they can be clearly differentiated from others making only a very dubious claim to such consideration. The involvement of mathematics, after all, is not a distinctive mark of science. Biology has for long been a science, though in it mathematics is but slightly brought into play; astrology is often highly mathematical without being at all a science. Hall remarks that:

It is possible to derive [astronomical] predictions from purely mathematical procedures, as the Babylonians did, without making any hypothesis concerning the mechanism involved.

Here precisely is the point. Just that concern for mechanism signalizes the appearance of what is recognizably science. In man's history this is a late development-coming first, says Schrödinger, not with the Babylonians or Egyptians but with the Ionian Greeks, in the 6th century B.C.

The Egyptian surveyor possessed and used a number of relations connecting distances, areas, shapes, and the like. Though often highly sophisticated and precise, these relations are not unreasonably associated with common sense: they remained discrete, unorganized, each in itself a separate enigma. The situation is changed through the studies of a long series of Greek investigators, culminating with 
Euclid. Postulating certain axiomatic relations between certain postulated "entities" (point, line, plane, etc.), Euclid shows that deduction from the axioms yields the many relations used by the Egyptian surveyor, and a number of other such relations not previously recognized. This system thus handsomely discharges the three major functions-correlative, explanatory, and heuristic-characteristic of a scientific theory. Only in its comparative perfection is Euclid's geometry atypical of the Greek achievement. In Greek astronomical theories, for example, we find again a large-scale co-ordination of hitherto separate relations (used in time-keeping, navigation, calendar-construction, eclipse-prediction, and the like), again an indication of certain new relations for which to look, and again a most impressive sense of explanation. Of this kind of achievement Margenau writes:

. . a theory was born; the surface of mere correlation was broken, subsurface explanation had begun. To put it another way: The contingency of correlation had given way to logical necessity.

Typically gratified in colligative relations like those used by the Egyptian surveyor, what I call the "Egyptian desire" evokes the intensely practical common-sense quest for order on which prediction can be founded. The new element that produces science-what I call the "Greek desire"-evokes a search for theoretic explanation of those relations, pursued urgently even when it offers no promise of improving the predictive capacities with which we are already endowed by the several relations. Far overshadowing the impressive though imperfect theoretic explanations the Greeks actually propose, is their first bold conception that such explanation is possible!

The principle of intelligibility. The "Greek way" turns on a new principle-distinct from those inherited by science from common sense-asserting the world comprehensible by man. Superficially innocuous, this view is revolutionary. Consider one example. Does not the divinity essential in a god render that god and his activities incompletely intelligible to mortal men? For, says Coleridge, "Incomprehensibility is as necessary an attribute of the First Cause as Love, or Power, or Intelligence." Accepting the principle of intelligibility we then recognize that the gods must not figure in the postulates of physical theories. Expecting to find physical phenomena intelligible, we seek to see them effects of natural (as distinct 
from supernatural) causes-and so arrive at the resolute naturalism characteristic of the best Greek thought.

Such naturalism in turn profoundly affects our view of the principle of determinism; for, after all, that principle may be viewed in many unequally "respectable" ways. A malignant demon, or a witch's curse, may reasonably be postulated as the hidden determinant of a death for which no overt determinant can be detected. Hard-headed Greek potters attached grotesque masks to their kilns, to frighten away unseen demons postulated to account for the otherwise inexplicable failure of certain entire firings. The idea of determinism may even be made into an argument for divination, as shown in the exposition of Stoic doctrine quoted by Sambursky:

. . . in every field of enquiry great length of time employed in continued observation begets an extraordinary fund of [common-sense] knowledge, which . . makes it clear what effect follows any given cause, and what sign precedes any given event. . . . the universe was so created that certain results would be preceded by certain signs, which are given sometimes by entrails and by birds, sometimes by lightnings, by portents and by stars, sometimes by dreams, and sometimes by utterances of persons in a frenzy. And these signs do not often deceive the persons who observe them properly. If prophecies, based on erroneous deductions and interpretation, turn out to be false, the fault is not chargeable to the signs but to the lack of skill in the interpreters.

The failures of divination here receive the treatment we ourselves give to, say, failures of the conservation of mass in the experiments of novice chemists. We take the reliability of the conservation law as a matter of principle; just so one can take divination also as of principle. Nothing about the principle of determinism as such thus enforces rejection of divination, and what we regard as similar superstitions. From the Greek postulate of intelligibility, however, there can arise rejection of divination: we reject as inadequate a determinist order we cannot understand.

The multi-faceted principle of intelligibility, elsewhere treated at length, underlies the entire attempt to construct theoretic explanations of colligative relations. Today notable successes already won in such construction encourage confidence in the principle-which we take wholly for granted. But for the Greeks the principle was no more than a daring assumption-and we honor them most for that indomi- 
table faith in the capacities of human reason which is an essential precondition for the emergence of science.

\section{SOME DIFFICULTIES}

Though science be here created, something more than the infusion of the principle of intelligibility into common sense is required to make of science a going concern. Impressive though it is, ancient science is science still incomplete, imperfect, incapable of self-perpetuation.

The choice of problems. Ancient science suffered simply as a pioneering enterprise, bound to take wrong turns in a still trackless wilderness. Over and over again it spent itself in attacks on what we recognize as trivial and/or intractable problems. Amply perceptiveand, in our view, thoroughly wrong-was, for example, Aristotle's feeling that mortal man is best advised to seek scientific understanding of equally mortal plants and animals, and notably ill-equipped to understand the apparently immutable rocks. Scientists can learn to choose better problems only as science acquires a history. Thus, given some two millennia of intermittent effort, continued failure to solve certain of the problems broached by ancient science becomes testimony that these problems might better be abandoned or, at the very least, subjected to the kind of drastic reformulation the problem of motion received at the hands of Galileo.

The technology of the Renaissance world in which science was reborn is relevant in this connection. Practical ballistics, for example, raised in new form, and with new urgency, the classical problem of motion. Retrospectively we see that many such problems derived from technology were "good" problems: i.e., feasible of solution, and with solutions rewarding in insight. Whatever the balance of the historic and technologic factors, accession of a worthier set of problems did put modern science on the roads to advances denied its ancient progenitor.

Heuristic tools. Ancient science picked the "wrong" problems and, again typical of a pioneering venture, had inadequate tools with which to treat them. Lacking experimental tools, it lacked even more acutely intellectual tools. If Empedocles identifies the two major forces of the physical world with Love and Hate, or if Aristotle (among others) constructs a system in which teleology is the prime motive power, who can find this surprising? What else could be expected of men who had a penetrating insight into human motivation 
but no clear concept of a simple machine? Who will conceive a clockwork universe before a clock is invented?

Again a more fully developed technology helps to get things moving. Beyond providing an abundance of materials and devices, serviceable to science as experimental tools, the mature technology of the post-Renaissance period opened the way to new conceptual tools. On a new wealth of industrial experience was founded a new and (in the issue) powerful group of mechanistic concepts and analogies, as alternative to the limited and limiting group of animistic concepts and analogies used in ancient science. Through history, once again, earlier errors become recognizable, and so avoidable. Nothing signified a priori the inappropriateness of the alchemists' conception that chemical change is most significantly characterized by the changes in color accompanying it. More than a millennium of patient but fruitless endeavor does convey some impression of such inappropriateness, and so encourages a search for new and better concepts.

Organization. The preservation of scientific history requires some organization. For this will suffice organizations external to science -Islamic library or Christian monastery. But history itself teaches us how much more fundamental is the need for organization within science. In the ancient world there existed no generally accepted scientific tradition, practically no stable scientific institutions (the Alexandrian Museum is a notable but late exception), and very few persons working as scientists at any one time. Different workers, largely self-educated, disagreed about the fundamental tenets of the science then cultivated by each in his own way. Men asked wholly different questions, and found for them wholly different answers. The work of each had then little relevance and often, because of terminological uncertainties, little meaning for the others. Ancient scientists were by necessity, if not by inclination, thoroughgoing individualists. Ancient science faltered, failed, because it lacked cohesiveness.

From its rebirth modern science could draw on organizational possibilities unknown to the ancients. In stable academic institutions like the University of Padua a tradition might be maintained in a library, and interpreted by able teachers to students who, in their turn, would become teachers of their successors. All the universities rested on one common foundation in scholasticism. From Oxford and Cambridge to Paris and Padua we find important differences in detail, but al- 
ways a common point of departure in a terminology and a set of premises shared by all. No matter how far the scientist might voyage in thought, he remained in touch with contemporaries who, moving perhaps in other directions, shared always the same base of operations.

Quite aside from the universities, men informed by the same tradition and sharing the same enthusiasm might band together, as in the Accademia del Cimento and the Royal Society. Such local organization was supplemented by a rapid transmission of intelligence along channels earlier developed for mercantile, ecclesiastic, and political purposes, a transmission also newly and dramatically broadened by the invention of printing. Within the 150 -year period embracing the publication of the works of Copernicus and Newton-and the birth of modern science-an association of scientists as pervasive as it was loose, and as powerful as it was intangible, invested science with a cohesiveness as crucially important as it was then novel, and today commonplace. The extravagant dissipation of effort so marked in ancient science is, as Herschel notes, already replaced by something of that co-ordination of effort $w e$ take for granted:

. . . the sparks of information from time to time struck out, instead of glimmering for a moment, and dying away in oblivion, began to accumulate into a genial glow, and the flame was at length kindled which was speedily to acquire the strength and rapid spread of a conflagration.

The social setting. Today science so thrives, in a culture we take wholly for granted, that some among us are led to the absurdity of supposing science independent of its social setting. Historical perspective rectifies that judgment: even the general organization of society can affect science. Ancient society was, for the most part, highly stratified. Possibly such stratification impairs communication between scholar and craftsman; probably it will suggest to the former that higher truths must be sought through contemplation, not through vulgar manipulative techniques. Often there may be also a crucial disparagement of the basic principle of intelligibility: in a highly stratified society supernaturalism, convenient superstitions, may have a weighty social role. But any serious concern for the social expediency of supernaturalism cannot but endanger grasp of the principle of intelligibility. Ultimately, for whatever reason, all hold is lost on 
that principle. (The Dark Ages are, in this respect, not inappropriately so termed.) Accepting a cosmology in which the world is base, and in itself of no importance, a man will in any case feel far more concern to purify his soul than to acquire the "vain knowledge" of which he supposes himself incapable. Science could then be reborn only as, in fact, it was-in a world that had acquired a deep new interest in man, in human life, and in the terrestrial habitat of man; that had acquired, too, a deep new faith in human capacity for knowledge. Langer observes that long before science could produce cultural change it had in part to be produced by such change:

... it was far less the information men acquired that undid their religious beliefs than the change of heart which prompted such research. The desire to construct a world-picture out of facts superseded the older ambition to weave a fabric of "values," . . .

The principle of intelligibility so reborn is of course in perfect harmony with the spirit of the Renaissance: not simply measure, man is to be also measurer of all things. Moreover "man" is not some fabled sage of antiquity, but modern man. The newfound confidence of man in himself was buttressed by the recovery of ancient learning. The ancients, so long supposed to have attained perfection of possible knowledge, were then found to have differed sharply among themselves. This previously unsuspected disagreement seemed amply to sanction the disagreement of modern man with previously accepted ancient doctrine. Such sanction was crucially important to many, and not least to Copernicus. One need then no longer be content to patch the Ptolemaic system to maintain a predictive device and to "save the phenomena." Kepler's laws are born of the effort not simply to describe and predict but to understand the mechanics of the heavens. With new faith in the possibility of new progress in human understanding, science takes a new turn.

The idea of progress. The idea of progress that we take so much for granted is a very considerable novelty. A few in antiquity conceived at least the limited idea of a continuously progressive science. Discussing a newly discovered mathematical theorem on the volumes of regular solids, Archimedes (quoted by Sambursky) says:

I am persuaded that it will be of no little service to mathematics; for I apprehend that some, either of my contemporaries or of my successors, will, by means of the method when once established, be 
able to discover other theorems in addition, which have not yet occurred to me.

Also quoted by Sambursky is Seneca, writing on cometary theories:

The day will come when time and the researches of long generations will bring to light what is now concealed. A single generation is not enough for the solution of such great problems. . . . Let us be content with what we have discovered so far. Those who come after us will also add their share to truth.

These statements, so true to the spirit of modern science, express what was in the ancient world a comparatively rare insight: the general idea of progress was not yet established-by most, not even conceived. No trace of that idea can be found in Aristotle's opinion that, rather than damage the prestige of all law, it were better to leave unchanged an admittedly bad law. There are indeed all too many ancient spokesmen for the medieval view of succession not as evolution but devolution; a long inexorable corruption and decay-from antique perfection of world, man, and knowledge-to final and soon-to-be accomplished dissolution.

In the ancient and medieval periods the world is then something to be endured, not a place in which improvement is to be expected or even sought. Haggard writes:

The medieval Christians saw in childbirth the result of a carnal sin to be expiated in pain as defined in Genesis III:16. . . . During medieval times the mortality for both the child and the mother rose to a point never reached before. This rise of mortality was in part the consequence of indifference to the suffering of women. It was due also to the cultural backwardness of the civilization and the low value placed on life. It was aggravated by the increasing difficulty attending childbirth. These were the "ages of faith," a period characterized as much by the filth of the people as by the fervor and asceticism of their religion; consequently nothing was done to overcome the enormous mortality of the mother and of the child at birth. It was typical of the age that attempts were made to form intrauterine baptismal tubes, by which the child, locked by some ill chance in its mother's womb, could be baptized and its soul saved before the mother and the child were left to die together.

Highly overcharged this account doubtless is. Yet the ingenuity that devised intrauterine baptismal tubes might not have been wholly 
incompetent in the design of obstetrical forceps. Why was this ingenuity not so deployed? Clearly because man had not yet grasped that something can be done, should be done, for the betterment of human life.

Bacon is not quite the first to argue that by learning to understand the world we gain power to change it; but the general idea of progress does not emerge in recognizable form much before 1600. A cosmologic idea, it has a technologic root. When little if anything else in the world seemed even compatible with the idea, technology actively encouraged it. A crude pre-scientific technology, still almost wholly dependent on cut-and-try empiricism and individual inventiveness, had brought forth notable improvements in mining and metallurgy, the horse collar, wind- and water-mills, medicinal distillates-genuine wonders!

However evoked, the idea of progress is absolutely vital to science, and no society lacking the idea (as did the great civilizations of the East) has ever supported a flourishing science. The idea is vital because without it the principle of intelligibility is-if not wholly discredited-seriously misunderstood. The most fundamental weaknesses of ancient science did indeed develop from overextension of the primary source of its strength: that same principle of intelligibility. On its rebirth in the modern world, the principle comes to be associated with three essential qualifications.

\section{A FIRST QUALIFICATION: PATENCY, PROGRESS, AND PRAGMATISM}

Physical theories "explain" colligative relations in terms of theoretic postulates themselves unexplained. Taking the principle of intelligibility in too extreme a form, I shall seek to close this final gap in understanding; and I may think to have found, in Euclidean geometry, the way of doing so. Euclid's postulates do not themselves seem to require any explanation, and I may so be led to stipulate that all postulates of all physical theories must be similarly "self-evident." If I do so stipulate I restrict, and perhaps even deny, the possibility of progress. Self-evidency is, after all, no more than conformity with the opinions of a day. Progress is then possible only when I feel free to entertain postulates that are not self-evident. These, if they lead to an attractive theory, may tomorrow be appraised-in terms of altered presuppositions and prejudices-as new self-evidencies. 
Lacking grasp of the idea of progress, the Greeks (with such notable exceptions as Aristarchus) showed comparatively little willingness to consider theoretical postulates that could not be made to seem self-evident. Such willingness represents a comparatively recent cast of mind. It emerges very gradually-it really struggles forth-in the 16th and 17th centuries. The multiple motions of the earth postulated by Copernicus are no self-evidencies, but Copernicus still feels bound to justify them by placing a strong emphasis on the self-evidency of other parts of his system: e.g., the perfect circular motions of celestial bodies, troubled by no such shabby evasion as the equant; the appropriately central position of the sun, the majestic giver of heat and light to our world. Almost a century later Descartes still thinks to construct a theoretic system by selecting as postulates only those to him both clear and indubitable, but in this Descartes' is an attitude more ancient than modern. What was to become the modern attitude is most vividly expressed in Newton's system. Here the postulates (e.g., of a universal gravitational attraction propagated through space) were defended on no appeal to this or that selfevidency. They were defended quite forthrightly on a newly fundamental plane: they work. Thus we are taught a more modest interpretation of the principle of intelligibility. We learn to content ourselves with explanations in terms of theoretical postulates not necessarily self-evident, and so gain power to work with a far greater range of possibilities than before. Believing in scientific progress, we are confident that-if never "self-evident"-our postulates will some day be explained, though then only in terms of still more fundamental postulates themselves unexplained.

\section{A SECOND QUALIFICATION OF THE PRINCIPLE OF INTELLIGIBILITY}

The principle of intelligibility may lead men to the inordinately optimistic expectation that all aspects of experience-physical, biological, aesthetic, mystical-can be promptly rationalized within one immense system. Thus Aristotle seeks to build a final system, in apparently complete confidence that the fullest understanding achievable by man can at one stroke be won. Thus Epicurus' atomism is for him not only a comprehensive physical theory but also a cosmology and an ethic. Not all of the ancients pursued such grandiose aims: in such specialized works as the geometry of Euclid, the statics of 
Archimedes, the pneumatics of Hero, and the astronomy of a Ptolemy we find what are for us the most important advances made by ancient science. But as long as a great system like Aristotle's is not obviously bankrupt, it is by far the most impressive; and so diverts effort from the apparently more pedestrian endeavors we see as having been better advised.

Almost two millennia after Aristotle his system is reconstructed by Aquinas with no reduction in scope of correlation. Even well after the beginning of the modern era Descartes supposes that he himself can build (or, later, could have built) a complete and final system. But in this same period, and even earlier, a newly modest conception of the principle of intelligibility was slowly gaining ground. Only as it did gain ground does modern science become possible.

Copernicus must brush aside the gigantic system of Aquinas to create a new system for astronomy only. To make a gain in local correlation, he is prepared to pay the price of an enormous loss of general correlation, produced when astronomy is thus separated from physics, metaphysics, and the like. Galileo also pays that price when he studies kinematics as a separate subject-distinct from others with which it had been allied by Aristotle. The study of the atmosphere, and of pneumatics in general, becomes similarly an area in which local correlation is sought, and won, as a prize for which one paid by relinquishing the general correlation afforded by the Aristotelian conception of horror vacui. Men learn to content themselves with local correlations of data, and to regard a general system as the remote goal of a long and difficult journey. The Newtonian synthesis clinches this approach by showing that, from tight local correlations, one can, ultimately, journey on to a system of great comprehensiveness.

Autonomous science and sciences. Grasping the principle of intelligibility, science emerges as a fully self-conscious endeavor to find rational order in experience. This is a science clearly affiliated with philosophy. Greek science was indeed closely (and disastrously?) associated with Greek philosophy. Even on its first appearance in the modern world, science is still "natural philosophy." Yet if the noun implies participation in the concerns of philosophy, the adjective already reflects a partial separation which, in time, is widened. "Natural philosophy" gives way to "science." If, as Bacon supposed, science 
is the "handmaiden of theology," she has yet a life of her own. This new autonomy is of prime importance. Science feels free to reject as subject matter much that concerns theology and philosophy: for this and other reasons the problems of science often can be solved as many of those of theology and philosophy cannot. Moreover, many then "extraneous" considerations cease to trouble the affairs of an autonomous science. Investigators who differ profoundly in their metaphysical convictions can still collaborate in science. In defense of the germ theory Pasteur, devout Roman Catholic, makes common cause with Tyndall, an atheist in the eyes of most of his contemporaries.

The separation of an autonomous science is followed by the separation of semi-autonomous sciences, and then of specialties within these. Scientific advance assumes a new pattern. At a number of distinct growing tips purely local correlations are projected outward. Larger correlations come later, as the separate shoots are linked through the same branch, and the several branches come (perhaps) to depend from the same trunk. Impatient to advance, we set aside this pattern only at detriment to advance.

The ancient scientist aspired to a general system embracing much if not all of his experience. He had then some fear of making radical separations of that experience: separated "parts" might not again fit together in a whole. But, reading what seems to be the lesson of scientific history, we venture quite drastic separations of elements of our experience. We become bold enough even to subdivide a unitary "phenomenon" for consideration in different disciplines. We observe the flight of a pigeon. Physics (aerodynamics) tells us something of how the beating of wings sustains motion in air, physics (kinematics) affords us a very simple description of the motion of the bird when it is shot, and physics (energetics) makes us aware that further problems remain to be solved. We may turn then to chemistry and biochemistry to learn something of the mechanisms of power production in birds. To biology we leave the problem of how a bird of this particular sort has come to be; to psychology the problem of why the bird elects to fly when and as he does. We deploy the concept of dissolubility more aggressively as we gain confidence in its natural complement. Accepting a principle of superposition, we take it for granted that parts will ultimately fit together in a whole. With faith 
in such progress, we look to make small gains, in expectation of greater. And we do gain where, hoping to win everything with one dramatic insight, the Greeks won little.

\section{A THIRD QUALIFICATION OF THE PRINCIPLE OF INTELLIGIBILITY}

The Greeks made observations, even experimerts, but most often experiments meagre in number and observations needlessly crude. Profound stimulus, the principle of intelligibility can be also snare. Overconfidence in the supreme power of human intelligence breeds a dangerously arrogant rationalism. When they already "know" the answer men make crude observations, if they make any. Even when they do not already have an answer, rationalists will suppose they require only a few observations to suggest the axiomatic propositions that yield, by rigorous deduction, definitive theoretical answers to all questions that can be asked. More than a millennium after Greek science died, Descartes still considered that a few experiments should suffice to reveal the system of the universe-experiments being necessary only to establish which of a few comparably self-evident alternate propositions the Creator had decided to actualize.

To conceive necessary only a few observations, or experiments, is far from the worst conclusion rationalists can reach. For how may one best seek the transcendent reality of that Platonic world of pure ideas, access to which demands all a contemplative reason can supply? How better than by turning one's back on the manifold distractions of the gross material world of the senses, where the pure ideas can at most manifest themselves only as distorted projections in a crude pattern of shadows? "They who wish to approach astronomy correctly will," says Plato, "turn their backs upon the heavens." On such a firm denial of the relevance of sensory experience can be founded nothing approximating science as we know it.

Modern science begins with a newly modest empirical emphasis for which Francis Bacon is spokesman:

There are and can be only two ways of searching into and discovering truth. The one flies from the senses and particulars to the most general axioms, and from these principles, the truth of which it takes for settled and immoveable, proceeds to judgement and to the discovery of middle axioms. And this way is now in fashion. The other derives axioms from the senses and particulars, rising by a gradual 
and unbroken ascent, so that it arrives at the most general axioms last of all. This is the true way, but as yet untried.

Is this second "way" so different from that recommended by Aristotle?

The natural path of investigation starts from what is more readily knowable and more evident to us [presumably the observable particulars], and proceeds to what is more self-evident and intrinsically more intelligible [the first principles, or Bacon's "most general axioms"].

Bacon's criticism seems aimed less at Aristotle's maxim than at its interpretation by Aristotle's followers. Taking their departure from a very small body of roughly observed "particulars," many in the Aristotelian tradition expected to arrive promptly ("flying") at first principles. The premature hardening of these can then develop into the first "way" criticized by Bacon: if some particulars incidentally observed seem out of keeping with the general principles, these particulars are simply dismissed-perhaps as illusions, trivia, errors, or imperfections of an intrinsically corrupt world.

The appeal to experience. The warm regard for observational particulars that Bacon preaches is far from a complete novelty of modern science. That regard informs the ancient and vital tradition of Hippocratic medicine. Long before the modern period the multiplication of epicycles in the Ptolemaic system testifies to continued efforts to take account of observational particulars. But with the beginning of the modern era the attitude Bacon commends assumes a new weight -perhaps, as Collingwood suggests, through the development of a new cosmologic conception of nature.

In the early phase [of Renaissance cosmology], the world of nature, which is now called natura naturata, is still conceived as a living organism, whose immanent energies and forces are vital and psychical in character. . . . as time went on . . . the idea of nature as an organism was replaced by the idea of nature as a machine. . . . But even the earlier view differed sharply from the Greek theory of the world as an organism, owing to its insistence on the conception of immanence. Formal and efficient causes were regarded as being in the world of nature instead of being (as they were for Aristotle) outside nature. This immanence lent a new dignity to the natural world itself. . . . it led people . . to look at natural phenomena 
with a respectful, attentive, and observant eve; that is to say, it led to a habit of detailed and accurate observation, based on the postulate that everything in nature, however minute and apparently accidental, is permeated by rationality and therefore significant and valuable.

Whatever its source, a new attitude is already clearly working in the founders of modern science. Consider Tycho. Brahe. Dissatisfied with the Copernican system, Tycho conceives that a definitive astronomical system can be founded only on highly accurate observational data. And he spends his life, as perhaps no man before him had spent his, developing superior observational instruments and compiling an enormous array of meticulous observations. So to spend one's life is to see the world of experience as-far more than a mass of misleading imperfection-the most essential foundation for the construction of the conceptual world.

Tycho was more than a little mad; but the same regard for the details of experience rules also the very different madness of the inheritor of Tycho's data. Johannes Kepler was a Pythagorean mystic -a classic seeker after the harmony of the spheres-and yet not quite of the classic mould. The labor of years finally permitted Kepler to fit Tycho's observations of Mars to an orbit which was at no point more than $8^{\prime}$ of arc out of agreement with observation. This trivial discrepancy-recognizable as a discrepancy only because of the enormous improvements in observational techniques made by Tychoany mystic before Kepler would have dismissed without a second thought. An ideal orbit coming this close must be sound; the "insignificant" deviations indicate no more than inadequacies in the data and/or the crudeness of the world to which the data refer. How different was Kepler's attitude!

Divine Mercy has given us in Tycho an observer so faithful that he could not possibly have made this error of eight minutes. We must thank God and take advantage of this situation; we must discover where our assumptions have gone wrong.

Kepler came so to a step wholly unprecedented: rejecting orbits compounded from circles, he sought a less "ideal" orbit that would better fit the data.

What was to become the new scientific attitude did not of course spring full-blown with Tycho and Kepler. Only slowly does the appeal to brute experience come to be established as the commonplace 
it is today. It could become established only as confidence in pure rationalism was sapped. But so it was. To some others, as to Bacon, the purely rational endeavor of scholasticism seemed to stand for stagnation, particularly in view of the notable advances made in technology by aggressive empiricism. And if the new empirical emphasis seems antirational, then so much the worse for rationalism; if empiricism promises progress, empiricism will be the choice of men intoxicated by the thought of progress.

A new conception of scientific method. Descartes, builder of a classic "system," envisions science as primarily an enterprise of rationalism. Bacon envisions something closer to natural history than to science when he puts primary emphasis on empiricism. Neither fully grasped the form modern science has assumed. Today the balance of abstract theory and concrete fact makes them essentially coequal: both are indispensable, either may be in error, each controls the other, and both are equally worthy of earnest attention and strenuous effort. Unlike his contemporaries Bacon and Descartes, Galileo proposes no formal method. But Galileo helps to set the style of modern science by presenting in his work an illuminating expression of what Hall remarks to be a method at once duly theoretical and duly experimental.

In the Dialogues and Discourses the foundations of scientific knowledge are shown to reside in phenomena and axioms conjointly. By its attention to actual phenomena Galilean science was made real and experiential; by its use of the capacity of the mind to apprehend axiomatic truths its logic was made analogous to that of mathematics.

Galileo set off down an indistinct track traveled by a very few among the ancients, but among them he whom Galileo most admired: Archimedes. With the aid of such concepts as the ideal lever, Archimedes had shown how, by abstraction, one could pass from the confusions of actual phenomena to "purified" phenomena in an ideal universe of mathematical discourse. With the aid of such concepts as "free fall," in an ideal realm without air resistance, Galileo sought to follow Archimedes into that same mathematical universe. Though then supported by a mathematics far more powerful than any known to Archimedes, the abstraction to mathematizable systems we take wholly for granted still remained in the age of Galileo the extremely difficult feat sketched by Butterfield: 
. . even when men were coming extraordinarily near to what we should call the truth about local motion, they did not clinch the matter-the thing did not come out clear and clean-until they had realized and had made completely conscious to themselves the fact that they were in reality transposing the question into a different realm-they were discussing not real bodies as we actually observe them in the real world, but geometrical bodies moving in a world without resistance and without gravity-moving in that boundless emptiness of Euclidean space which Aristotle had regarded as unthinkable.

And now arises the crucial question-posed, and answered, by Hall as follows:

When abstraction has played its part, when attention has been given to the really existing physical properties of bodies, when the mathematization of the phenomena has been fully explored and a theoretical science begins to take shape, how is the investigator to determine whether his image or model of things in the abstracted universe represents faithfully things as they are according to experience? Galileo's answer to this problem, prepared for him by earlier logicians, was the appeal to experiment. If theoretical examination suggests that in specified conditions the event $\mathrm{B}$ will follow the event $\mathrm{A}$, then the reasoning can be tested by creating those conditions, and making the observation.

By analysis of a collection of varied observations and experiments, Bacon supposed, the scientist strips away successive veils of deceptive appearance to arrive, at last, at a hard core of secure knowledge. Stripping away, one by one, the concentric coats of an onion, we so arrive at last at its center. This metaphor itself suggests the inadequacy of the Baconian conception: working entirely from the outside, detachment of the onion's smooth and tenacious shells is very difficult. We succeed precisely when, penetrating with knife or fingernail, we secure the purchase with which we detach the coat by breaking outward, toward the surface. The successful technique in the metaphor suggests the quadripartite scientific method pioneered by Galileo (and Archimedes):

(1) Resolving to study "falling bodies," the scientist finds that he can hold simultaneously in view very few unrelated elements of experience. Quite early in his enterprise he must then devise a body of 
(appropriate, he hopes) concepts-e.g., velocity and accelerationwhich invest certain selected (significant, he hopes) elements with relatedness. A considerable abstraction is already expressed in the selection, and a much greater one is now to be made.

(2) With bundles of data bound by his concepts, he withdraws from the complex world of experience toward an "ideal" conceptual realm. Refusing to accept the limits common sense sets itself, the scientist deals quite elaborately with refined abstractions akin to those of the philosopher. He seeks to penetrate behind the appearances: he hypothesizes existent in nature certain simple entities and relations (e.g., "free fall"). In terms of these he asks certain new questions, perhaps comes to grips with the core of his problem. Always he seeks for ever greater abstraction, thinking thereby to penetrate ever more deeply-to the essence of an ever wider body of phenomena.

(3) How can he gain confidence that his abstract conceptions do hold any such essence? Unlike the ancients, he rejects the expedient appeal to self-evidence. He tries instead to break back out to observables: drawing deductions from his abstract postulates, he ventures to predict what, under certain circumstances, should be found in actual experience. Thus always in the end the scientist returns from the world of abstractions, however remote, to the world of experience the man of common sense never fully leaves-and the philosopher never fully regains.

(4) The scientist need not return all the way to the raw experience of observation, but only as far as experience provided by laboratory systems purposefully designed to highlight the effects of the factors to which the abstract concepts direct attention. With Galileo we come then at last to the classic experiments on the inclined plane. The theoretic predictions being borne out, the theoretic construction may be sound. But were inexplicable predictive failures to be found, these-not to be brushed aside as marks of the nonideality of an intrinsically imperfect world-are to be recognized as calling the abstract constructions in question.

Considering our experience, we create in our thoughts a world of ideal abstractions. Whatever its beauty, this world is nonetheless an artifice of thought. Ultimately the business of science is to talk not of this world but of the "real" world, with all its imperfections and complexities. Experience thus becomes the touchstone by which we prove (in the sense of test) our abstract conceptions. 
A further small dash of Baconian empiricism is required to give Galileo's procedure a fully modern flavor. Thus Galileo himself is still too much the Platonist to accept Kepler's elliptic orbits in place of the "perfect" circular orbits that did not meet the observations quite as well. He is still too little inclined to pay attention to minute details of experience in a world clearly falling short of classic perfection; this is evident even in his account of the results of the celebrated experiments on the inclined plane. These results he reports as agreeing with each other, and with theoretical predictions, to a degree wholly unattainable with his crude equipment. Intending no dishonesty, Galileo clearly reports not the actual results, but rather what he confidently expected would have been the results if only things had been more perfectly arranged-ideal results to which he judged the actual results a sufficient approximation.

Galileo is not alone in such reporting, of course. Perier does much the same in reporting the results of the Puy de Dôme experiment, and Pascal reports many "experiments" patently not experiments done but demonstrations confidently conceived. In such instances we never know what are actual experimental results, what are idealized results of actual experiments, and what are only hypothetical results of hypothetical experiments. From this intolerable situation science escapes only through the emphasis made by such as Boyle on the absolute need to distinguish clearly between what is observed and what should be, might be, or could be observed. Such emphasis we find in Boyle's somewhat acerb commentary on Pascal's "results," as well as in Boyle's own incredibly tedious reports of his own results-down to the last trivial but possibly remotely relevant detail. Faithful Baconian that he was, Boyle is far too empirically minded. But in Newton's Opticks we find at last a very nearly optimal balance of theory and experiment, brilliantly struck by an Englishman who, influenced but not obsessed by the Baconian tradition, adopts the Galilean approach. We arrive here at that experimental science described by Weizsäcker:

Only the triad of thinking, acting, and perceiving makes the experiment possible.

It is not even sufficient that, in addition to perception, one of the two active dispositions is added to perception: only thinking or only acting. In the first case, the result is philosophy; the second, handicraft. . . Galileo . . . is probably the first to embody that unity 
which is no longer either philosophy or handicraft because it encompasses both; each of his manipulations is guided by thought, each of his thoughts by experimental evidence.

The Egyptian and the Greek inquiries come thus to complement and strengthen each other, and from their crossing emerges a modern science invested with true hybrid vigor.

The principle of corrigible fallibility. However we conceive science, we cannot begin our work without some facts and some ideas provisionally accepted as unchallengeable. But the ideas may be quite wrong; the facts ill-observed or laden with "credulity" (see p. 44). Obsessed by such fears we don't do science! From such obsession scientists are freed by their acceptance of what I call the principle of corrigible fallibility. It is a principle of action. Beginning with the best available facts and ideas, we proceed vigorously in the faith that any errors in them will be revealed by the interaction of facts and ideas-by the interaction of rational and empirical elements in neither of which individually we have, or can have, absolute confidence. We amend our hypotheses in the light of our experiments; but we also reject (as "errors"), "correct," and explain away some of our data when they conflict with "indubitable principles." In such unquestioning acceptance of principles to which all experience is made to conform (e.g., the conservation laws) science may seem at one with divination, or magic. Yet science progresses as magic does not, and not simply because science had the good fortune to hit on the "right" principles at the outset. It did not! But it learned better principles.

Bacon envisioned a "gradual and unbroken ascent" to the most powerful axioms, or first principles. The subsequent history of science demonstrates, however, that even a gradual ascent is not unbroken, that no principle remains forever beyond the challenge of experience. It is then far from facetious to describe the history of science as a tale of great and beautiful theories slain by ugly little facts-or, in Bronowski's phrasing:

Science is a great many things, . . . but in the end they all return to this: science is the acceptance of what works and the rejection of what does not. That needs more courage than we might think.

It needs more courage than we have ever found when we have faced our worldly problems. 
Perhaps this is mere vulgar pragmatism. But it is the attitude that has made scientific thought so rapidly progressive while human thought in other domains has been hobbled by inability to reject ideas that, however refined and attractive, too plainly don't work.

\section{The Body Scientific}

In the fable, the blind men's accounts amply suggest that the unseen "elephant" has differentiated parts. Just so, the extreme variability of modern accounts of the invisible abstraction "science" should prepare us to find complexity in what we ordinarily regard as an integral enterprise. The artisan, the statesman, and the proverbial man in the street almost invariably define science as something pertaining to technology. The theologian, the philosopher, and any other nonscientific scholar are most likely to define science in terms of cosmology: world-pictures of the mechanical universe, the evolving universe, the statistical universe, and so on. Asking scientists for the meaning of "science" we find that most do at least agree on what science is not. Almost all scientists draw a sharp distinction between science properly-so-called ("pure science") and the applications of science in technology; most will also distinguish science properly-socalled from the world-pictures of cosmology. Thus, for example, Weizsäcker urges on us the need

to distinguish between two meanings of classical physics: as a world view and as a methodological instrument.

Scientists are members of human society, and science is conducted within a general cultural atmosphere. Not science, but only a wholly fictional abstraction, lives in a social vacuum. How then is one to treat of "real" science without embarking on a general sociology? We can hope adequately to treat science as a separate entity if only we can first grasp the nature and effects of its various linkages with its social milieu. These interactions, all proceeding through the mediating enterprises of cosmology and technology, I examine in Chapter IV.

Undertaking an anatomization of "science," I begin by distinguishing it from two other enterprises intimately enough allied to bes confused with it, and it with them. The more detailed structure revealed, as the anatomization is now pressed forward, will be found 
to impose an organization on the remainder of the inquiry to which this book has been directed.

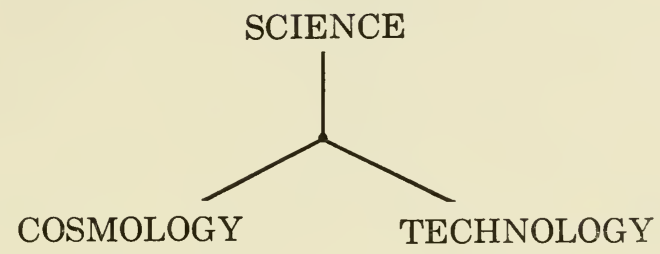

Even after we distinguish science from technology and cosmology, there remain differences of opinion on what we mean by "science." Conant notes two apparently contradictory opinions, qualified by him as the "static" and the "dynamic." In the static view we associate science with the fabric of scientific knowledge, as when Dingle writes:

Science is an achievement and not a method of achieving.

On the other hand, from considerations of probability theory Brown infers ". . . a tendency to diminution of scientific knowledge in the absence of further experimentation or confirmation"; and so adopts the dynamic view:

Left to itself, the world of science slowly diminishes as each result classed as scientific has to be reclassed as anecdotal or historical. . . . Science is a continuous living process; it is made up of activities rather than records; and if the activities cease it dies.

Consider that "work," "learning," and "research" have all dual usages, as verbs and nouns. "Science" is explicitly only a noun, but implicitly it shares a significant dualism of meaning with "learning," etc.-and even with its supposed opposite, "art." Art may be the activity of artists, but also the pictures, sculptures, etc. so produced. Holding a "dynamic" view of art, one can maintain that art is dead if no pictures are being produced. But holding that pictures are lasting achievements even when no more such pictures are being painted -adopting a "static" view-one speaks of Aurignacean cave painting as art. Recognizing the implicit dualism of "science," we obviate the apparent contradiction of dynamic and static views.

I do not complicate the diagram to indicate the dual meanings of technology and cosmology, but my representation fails seriously by 


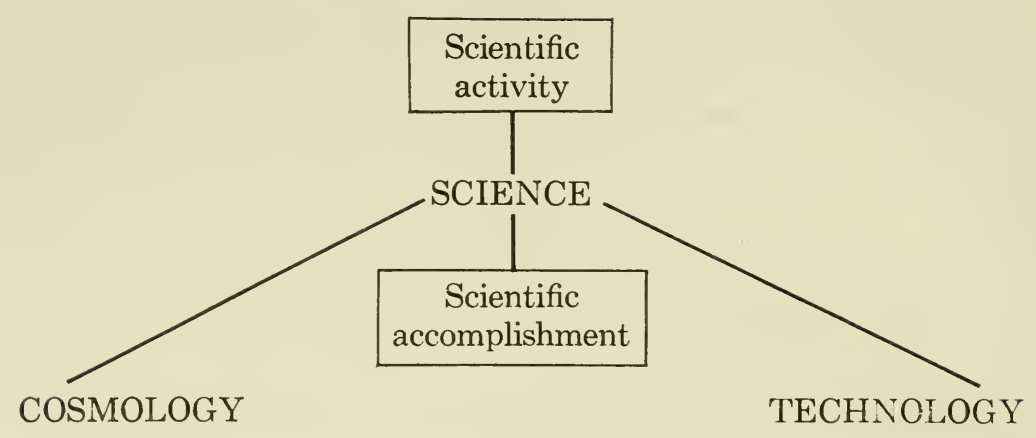

not distinguishing two distinctly different kinds of scientific activity. The activity of individuals-immensely diverse and apparently anarchic-is the highly personal expression of individual creativity. Such activity (examined at some length in Chapter XI) is stabilized, co-ordinated, and fostered by the activity of organized science. Science is to its core a social enterprise; neglecting this, we neglect elements that determine at a most fundamental level the nature and capacity of science. Most obviously, science deals only with experience at least potentially common to all whereas, say, art treats of experience that is highly individual. In the aphorism-L'art, c'est moi; la science, c'est nous-the dichotomy is drawn oversharply. But the poet has properly recognized the decisive role in science of collective activity, collective judgment, perhaps even collective wisdom. The nature and functions of organized science I consider in Chapter $\mathrm{X}$.

Even yet the anatomization is incompleie. Weizsäcker speaks of classical physics as a "methodological instrument." Clearly appertaining to the activity of science, a methodological instrument is not itself activity but, rather, one product of antecedent activity. We attain a more revealing picture when, just as we distinguished two kinds of scientific activity, we distinguish also two kinds of scientific accomplishment: see the diagram on the facing page.

The anticipatory apparatus. Temporarily ignoring some complex overlaps, we may say that in the main the anticipatory apparatus is the locus of a gargantuan assembly of colligative relations, or laws, investing us with powers of prediction. Here the Egyptian desire finds its consummation; and the conception of science as "accumulative knowledge," its most conspicuous vindication. Always our pre- 


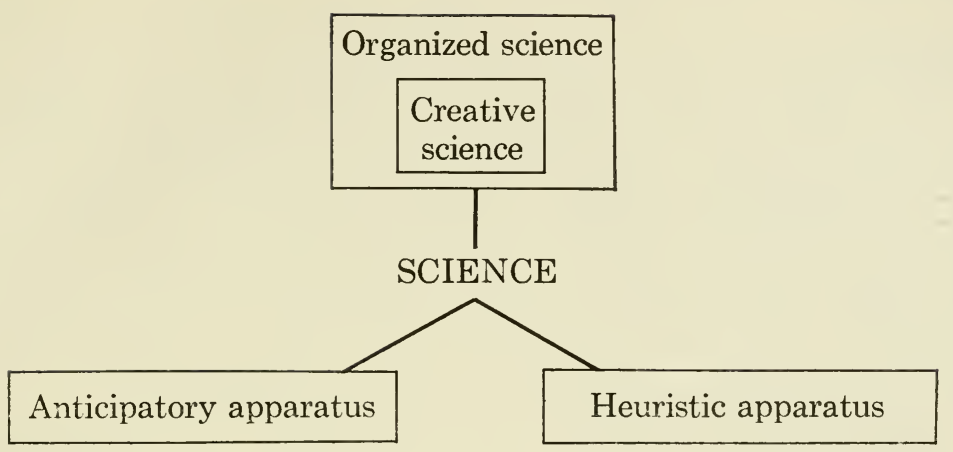

dictive capacities grow, always we find increase in the number of relations, and of the relations between relations sketched in theories here functioning as correlative devices. Dingle urges that:

. . . if, as we must surely do, we wish to characterize science by the element in it that persists and grows, and not by that which continually changes, we must recognize . . . the progressive discovery of relations between the various constituents of our experience, . . . Amid all the changes of theories and pictures and conceptions, the relations remain and steadily accumulate. Franklin found that lightning was a manifestation of the electric ether revealed in laboratory experiments. The electric ether has disappeared, and other theories of electricity have in turn succeeded it and disappeared also, but the relation between lightning and laboratory sparks remains. Maxwell established a relation between light and electromagnetic oscillations. His ether also has gone, but the relation stays. All permanent advances in science are discoveries of relations between phenomena, and the factor in science that shows a steady uninterrupted growth is the extent of the field of related observations.

'This effective immortality of scientific relations, together with some other of their characteristics, I examine in Chapter V.

The heuristic apparatus. The heuristic apparatus is the repository of tools for the winning of new knowledge. Though of great diversity, these tools fall naturally in two major groups. I imagine the heuristic apparatus bifurcated into two lobes. A conceptual lobe represents the tools of thought. An empirical lobe represents the tools of action -the instruments and devices, techniques and procedures, materials and specimens with and on which the scientist makes his observa- 
tions. The use and usefulness of these empirical devices I consider in Chapter VI.

An inventory of the contents of the conceptual lobe is difficult. Prominent here is a considerable group of methodological and substantive principles, examined in Chapter VII. Here too appear colligative relations in a variety of other guises, as well as a great array of concepts furnishing terms for the expression of theories and relations. Here also is found a heterogeneous stock (1) for the framing of analogies and the construction of models, and an enormous stock (2) of formal relations furnished by logic and mathematics.

Modern science arose after, and profited from, the development of mathematical tools the Greeks wholly lacked: $e$.g., the concepts of zero, probability, and functional relationship. The profits are enormous because advances in mathematics offer more than convenient new symbols and devices for the expression of colligative relations. They provide also immensely powerful new machinery for the construction of postulational systems. As alternative to the restricted possibilities of deduction in the syllogistic and Euclidean modes, we acquire a variety of others the importance of which first becomes manifest in the magnificent achievement of the Newtonian synthesis.

Much accelerated by growth of the formal stock (2), due to progress in logic and mathematics, the advance of science is probably even more profoundly accelerated by growth of the analogic stock (1). Once science achieves a competent development, and becomes capable of generating its own analogic stock, its further progress may thus be rendered auto-accelerative. That is, with more ample analogic stock scientific advance becomes more rapid, and more rapidly generates new analogic stock, as a result of which . . . and on and on. We find this effect even in the formation of laws. Ohm, for example, arrives at his well known law by treating the "flow" of electricity as analogous to the "flow" of heat previously treated by Fourier. Success thus feeds on success, as is even more apparent when we turn from laws to theories. The astronomers' conception of the solar system furnishes the analogy on which Bohr founds his conception of the nuclear atom with circumambient electrons; and that theory of the atom, completed in quantum mechanics, furnishes in its turn the analogy by which we conceive a "shell structure" of the nucleus.

However we appraise their relative importance, both the formal stock and the analogic stock clearly belong to the heuristic apparatus. 
In Chapter VIII I examine how theories are constructed by the association of logical or mathematical formalisms with models or analogies. Shall I then place theories also in the heuristic apparatus? As devices correlating scientific laws they belong in part to the anticipatory apparatus; as explanatory devices, designed to meet the Greek desire for understanding, they belong in part to the area of cosmology. Their status there I discuss in Chapter XII. But sharp separation of the correlative, explanatory, and heuristic functions of scientific theories is quite artificial. I find their heuristic function irreducibly dependent on their correlative and explanatory functions. Resolving to treat theories as heuristic devices, I can then feel confident they will be treated in all their aspects. One further consideration strengthens this resolve: an extended analysis of the complex criteria involved in the selection of scientific theories-set forth in Chapter IX-leads to the conclusion that heuristic power is the decisive criterion of selection.

Science, I argued, begins as an extension of common sense. The anatomy of science must then be present, if only in rudimentary form, in the anatomy of common sense. So it is. Common sense does comprise an anticipatory apparatus: a manifold of colligative relations. Common sense does relate to practical concerns like those of technology, and accepts uncritically (in its language) a cosmology. Like science, common sense develops through the work of creative individuals. The organization perpetuating common sense consists of all who speak the language of common sense; the organization perpetuating science involves all who speak the language of science. Where then are localized the differences that so sharply differentiate science from common sense? In the hueristic apparatus. In common sense the heuristic apparatus has so little formal development that ordinarily we do not even recognize it as a heuristic apparatus. The differentiation of science from common sense begins when a few new concepts (e.g., intelligibility) appear in the heuristic apparatus of common sense. Ultimately these additions make themselves felt throughout the anatomical configuration, and nowhere more strongly than in a heuristic apparatus itself become enormously hypertrophied. Possession of a highly elaborated organ for the winning of new knowledge then invests science with a dynamism unknown to common sense. 


\section{Cosmology and Technology}

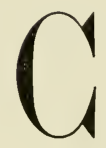

osMology and technology mediate the linkages of science with its social milieu. By way of some of the most obvious and direct of these linkages, scientific activity acquires a supply of problems, motivation, and logistic support.

Problems. Out of cosmology develop broad questions about the nature of the world and of man, parts of which have concerned scientists in all ages. These are Greek "Why?" questions, considerably reformulated when they become the concern of science. Much more limited Egyptian "How?" problems are broached by technologyand, more specifically, by its failures and inadequacies. These problems-of immense importance in the historical development of science -are also drastically reformulated as they become the concern of science. They are generalized, set in a much broader context, and most often treated with no immediate regard for technology as such. Practical difficulties in navigation, calendar-construction, and astrological forecasting initiate the development of scientific astronomy. A new science of pneumatics takes its departure from an unexpected limit of 34 feet on the capacity of lift pumps; a new science of thermodynamics, from an unexpected limit on the efficiency attainable with steam engines. Examined by Pasteur, certain problems of the fermentation industry lead to the foundation of a new science of bacteriology; in our own day troublesome "noise" in wireless transmission broaches a problem that ends only in the creation of the new science of radio astronomy.

Motivation. Believing in the possibility of enriching human life 
and alleviating its hardships, some men have sought and valued scientific knowledge read as technologic power. From Bacon to such as Bernal in our own day, these men have pursued science with technologic intent. By other men, from Kepler and Newton to such as Sherrington and Polanyi, science has been pursued and valued for its cosmologic significance. Science may then be conceived as the search for beautiful mathematical harmonies implicit in the cosmos and discoverable by man, or as the foundation of "natural religion." Today men may pursue scientific knowledge "for its own sake," but of course the valuation then set on "pure" learning is itself a cosmologic judgment.

Support. Like other humans, scientists need food and shelter; they need, in addition, the physical equipment their work demands. Until such time as science affords them financial support, men lacking independent means cannot easily function as scientists, and few of them will even study science. Probably the distribution of ecologic niches for scientists affects the direction of scientific endeavor; certainly the extent of the availability of such niches strongly affects the scale of scientific endeavor. Edelstein finds ancient science fatally weakened by lack of social support, but right up to 1800 there are very few posts for scientists, and very few who make their livings as scientists. Significantly enough, the word "scientist" is of coinage only. slightly more than a century old; only then did the existence of a substantial professional group raise the need for a group designation.

When the nonscientist supports science most often his interest is not in science as such, but only in science as contributor to the cosmology or technology in which he does take an active interest. Support given out of concern for technology appears quite early: the Ptolemys had quite practical reasons for supporting the Alexandrian Museum. On the other hand, such support dwindled to nothing during the Dark Ages in which man's attention turned from this world to another. As the modern era opened, experimental science gained a foothold in the universities as the affiliate of already established technologic and cosmologic concerns; i.e., as a subject studied by students of medicine and divinity, respectively. In this period Bacon preaches support of science both as the handmaiden of theology and as the great root of technologic advance. Bacon's estimate of the potential capacity of science to produce such advance far exceeded its actual capacity then, and for some two centuries thereafter. The 
situation today is entirely different and-if the funds given science with intent to benefit technology continue as abundant as they have become in the last twenty years-some early and drastic reorganization of the whole pattern of scientific endeavor seems well nigh inevitable.

\section{Cosmology}

The microcosmic life cycle of the liver fluke is, in the hands of a Sherrington, a subject as fit for cosmologic thought as the macrocosmic evolution of the spiral nebulae. More than inquiry into the wholeness of the universe, cosmology is the search for answers, and the answers given, to questions concerning the "real nature" of the world and of man, and even concerning the "reason" or "value" of the world and of man. Each scientist entertains a cosmology he does not, and perhaps cannot, wholly distinguish from his science. That important distinction is, however, often possible for the onlooker alert to four traits that characterize cosmology.

Inclusiveness. No concern of science, the "meaning" of human existence cannot but concern cosmology. Purporting itself a world view, cosmology (a "branch of metaphysics," according to Webster) must take in much that science rejects as unfit for its consideration.

Science metamorphosed. Quite differently incorporated in different cosmologies, science is there always metamorphosed in a fashion highly distinctive of cosmology. The concept "atom" (or "energy"), a useful tool of scientific thought, becomes then the "real stuff of which the universe is made." What have been found in science "suggestive" analogies, "permissible" principles, "sufficient" theories, "efficient" laws, and "appropriate" concepts are by the cosmologist all seen as "truths." A law known highly reliable is confidently assumed exact; known to be of great generality, it is confidently assumed universally applicable. As Bridgman notes, so much is true even in "purely scientific" cosmogony:

To me the most striking thing about cosmogony is the perfectly hairraising extrapolations which it is necessary to make. We have to extend to times of the order of $10^{13}$ years and distances of the order of $10^{9}$ light years laws which have been checked in a range of not more than $3 \times 10^{2}$ years, and certainly in distances not greater than the distance which the solar system has traveled in that time, or about 
$4 \times 10^{-2}$ light years. It seems to me that one cannot take such extrapolations seriously unless one subscribes to a metaphysics that claims that laws of the necessary mathematical precision really control the actual physical universe.

Disagreement. In our cosmologies we do not agree. On the merits of a purely scientific idea more than 25 years old practically all scientists are in substantial agreement; cosmologic issues now millennia old are often still the subject of acrimonious debate. Consider: humans, with their limited knowledge, can build a world-view only by relying very heavily on analogies. But analogies are less "given" than "felt": where I sense fundamental analogy you may find only trivial similarity, or even significant opposition. Consider further: prior to the "hair-raising extrapolations," our choice of what we will extrapolate determines for each of us the nature of his cosmology, and conversely. Some cosmologists, adopting an "organismic" view, choose to stress the continued failure to reduce all phenomena to mathematical expression. Others prefer to stress the great successes already won in the construction of mathematical laws and theories-successes the cosmologic connotation of which was read by the eminent scientist Jeans as: "God is a mathematician." The statistical form of certain scientific laws and theories is emphasized by some. The connotation of an abstruse mathematical theory may then be read as: "The uncertainty principle restores Free Will." An aliernate connotation, read with equal legitimacy but having a very different cosmologic flavor, is: "At bottom everything is ruled by blind chance." Other cosmologists may choose to emphasize the non-statistical form of certain relations: the integral laws (of least action, etc.) are taken by Planck and others to indicate a certain causal purposiveness in nature. But this conclusion can be combatted, as it is by Born, with the argument that:

The minimum principles are not duè to nature's parsimony but to human economy of thinking, as Mach said; the integral of action condenses a set of differential equations into one simple expression.

Rigidity and revolution. Because of its inclusiveness, my cosmology involves what are to me matters of deep personal concern. I am committed to my personal cosmology, and the hardening of my resolve to meet the perennial challenge of other cosmologies is mir- 
rored in a hardening of my ideas. As ideas become thus finalized as dogmas, even creeds, there yawns between cosmology and science the Jamesian chasm-"between categories fulminated before nature began, and categories gradually forming themselves in nature's presence." Founded on the principle of corrigible fallibility-denying to itself any claim to knowledge of "first principles"-science remains ever plastic, even to drastic change. In cosmology the possibility of change is commonly unrecognized, even denied: hard but brittle, a cosmology crumbles under the impact of new ideas, the effect of which is then plainly revolutionary.

Are there not "scientific revolutions"? Even a revolution in cosmology must have some secondary impact in science: the attitudes engendered by my personal cosmology influence the orientation of my efforts as scientist. But the discussion of "scientific revolutions" (in Chapter IX) leads to the conclusion that their most conspicuous trait is their un-revolutionary quality in science. Even without benefit of that discussion, one easily recognizes that in our actual usage the term "scientific revolution" designates solely certain episodes that were revolutions in cosmology. The works of Copernicus, Newton, Darwin, and perhaps Freud are commonly regarded as scientific revolutions. But the works of Lavoisier, Dalton, and Maxwell are rarely so regarded, though inferior neither in novelty nor in scientific importance. Why? In the first group of works each has very large cosmologic connotations; we attach no such connotations to the works in the second group.

As a scientific doctrine Copernicus' theory was no worse than ludicrous. Its immediate cosmologic impact was sufficient to rouse the wrath of Luther; its delayed impact was violently explosive. And observe that while Galileo was "only" severely rebuked for espousing the Copernican theory as science, Bruno was (in 1600) burnt at the stake for heresies nourished by its rich cosmologic implications, e.g., the infinitude of the universe and the plurality of inhabited worlds. Here, in cosmology and not in science, occurs the true Copernican revolution. As the directly contrasting case consider Lavoisier who, overthrowing the phlogiston theory, may be held to have worked a revolution. Indeed, chemical thought is by him changed as drastically as astronomical thought by Copernicus. However, while Copernicus is generally acknowledged to have worked a revolution in human thought, the very most we say for Lavoisier is that 
he worked a chemical revolution. Copernicus' work forced a reappraisal of the nature of the cosmos and of man's place in it; Lavoisier's work forced nothing of the sort.

With the name of Newton we associate a genuine revolution in cosmology. For example, the Newtonian conception of "natural law" leads through Locke and others to the outlook of the Age of Enlightenment. The elegance of the mechanism of the cosmos Newton himself regarded as absolute proof of the existence of God; but elaboration of the "world-machine" led inexorably to Laplace's conviction that, in celestial mechanics, the hypothesis of God is a superfluity. Consider now: in science Dalton's atomic theory did for chemistry, and Maxwell's electromagnetic theory for physical optics, very much what Newton did for physical dynamics. But Dalton's work or Maxwell's work is never called "scientific revolution"! Even the violent overthrow of the long-entrenched caloric theory by the kinetic theory (in which action Maxwell also distinguished himself) fails to win estate as "scientific revolution." Naturally! The effect in science of a theoretical advance is almost irrelevant to any claim on that title, the sine qua non for award of which is the production of a major upheaval in cosmology. This is precisely what was produced by the work of Darwin. Darwin destroyed no established scientific theory: indeed the idea of evolution (though not evolution by natural selection) had been "in the air" for more than half a century. Surely it is obvious that the genuine revolution in which Darwin figures (and in which, significantly enough, Huxley figures even larger) is a revolution in cosmology. And the discrimination expressed when we speak of a Darwinian or Newtonian (or Freudian) revolution-but never of a Daltonian or Maxwellian revolution-itself defines for us the domain of cosmology: it is the locus of primary impact of what we call "scientific revolutions."

\section{THE CLIMATE OF OPINION}

Contemplating the very irregular appearance of genius, scientific and otherwise, Kroeber hypothesizes that of many seeds of potential genius, produced sparsely but relatively uniformly in all places and times, only those few flower that are reared in the cultural environments of certain particular places and times. Consider, as an example, the astonishing galaxy of scientists who grew up in or near Budapest around the turn of the present century. Hungary had not earlier, and 
has not since, produced so large and illustrious a group as Hevesy, Polanyi, Szent-Györgi, Szilard, Teller, Von Bekesy, Von Karman, Von Neumann, Wigner, and many others. Perhaps the situation is as Duhem depicts it:

Contemplation of a set of experimental laws does not, therefore, suffice to suggest to the physicist what hypotheses he should choose in order to give a theoretical representation of these laws; it is also necessary that the thoughts habitual with those among whom he lives and the tendencies impressed on his own mind by his previous studies come and guide him, and restrict the excessively great latitude left to his choice by the rules of logic. How many parts of physics retain to this day a merely empirical form until circumstances prepare the genius of a physicist to conceive the hypothesis which will organize them into a theory!

However this may be, even quite local variations in the cultural environment may have spectacular effects. Consider what happened at the University of Padua. Here flourished a literally radical culture. Going back (by way of Averroes rather than Aquinas) to the taproot of scholastic thought, to Aristotle, it embraced also a Galenic element of respect for observation and experiment. The University had a strong secular orientation, buttressed by the dominion of the strongest anticlerical state then extant (Venice), and offered a freedom of thought unparalleled in any learned institution of the time. What a profusion of scientific genius is found among men whose outlook this University had moulded: Cusanus, Copernicus, Fabricius, Vesalius, Harvey, Galileo-all these and others, making up a substantial proportion of that small company which made modern science, had drunk the heady wine of Padua.

We found in Chapters I and II that perceived "naked fact" is, if not quite mythical, at least substantially hypothetical. We find now that, in confrontation of "naked fact," scientific ideas are born of human minds suffused with extrascientific, metaphysical presuppositions. Eddington indeed remarks that "because a man works in a laboratory it does not follow that he is not an incorrigible metaphysician." Consider for example how much the design and conduct of contemporary laboratory practice owes to a not-always-accredited cosmologic conviction that for every macroscopic physical effect observed there should be a discoverable "natural" cause. And, outside the laboratory, Eddington was himself a living demonstration of the 
enormous role that personal metaphysics may play in "purely scientific" matters when deeper theoretical judgments are involved.

This effect is obvious even in the routine deployment made of the principle of continuity. In company with common sense, science long accepted ideas of fabulous things and events occurring "strangely" in remote places and times. Until Kepler, Galileo, Descartes, and Newton, few indeed were those who dared to extend to the celestial realm the laws known to obtain in terrestrial contexts. Conversely, 19th-century physicists did not hesitate to extend relations, hammered out in the macroscopic world of experience, to the hypothetical microcosmic world underlying it. Today the timidity of the earlier attitudes and the confidence of the later both appear excessive, but of course both depend (like those "hair-raising extrapolations" of which Bridgman speaks) on essentially metaphysical presuppositions.

The cosmology of the individual scientist-uncritically formulated, unarticulated, and active at a level well below full consciousnessmust, as Crombie observes, make itself felt throughout his science.

.. . there has never been natural science with no preconception at all of theoretical objectives of a philosophical kind. . .

The procedures of science are methods of answering questions about phenomena; . . . But the form the questions take, the direction and extent to which they are pressed in the search for an explanation, will inevitably be strongly influenced by the investigator's philosophy or conception of nature, his metaphysical presuppositions or "regulative beliefs," for it is these that will determine his conception of the real subject of his inquiry, of the direction in which the truth hidden in the appearances will be found.

Beyond "promising" problems and "responsible" techniques, even what are "acceptable" solutions will so be defined. A science giving purposive explanations in answering "Why" questions yields to a science giving causal explanations in answering "How" questions; and this may, in its turn, yield ground to a science furnishing only functional relations, permitting answers to "What-When-Where" questions. All these sciences reflect cosmologic opinions, and are themselves mirrored in such opinions.

Commenting on the great pioneers of science, Polanyi remarks that "even their outlook will remain predominantly determined by the 
time and place of their origin." And, as regards scientists generally, surely an affirmative answer must be given to Weizsäcker's query:

Should we not say that the historical situation of human consciousness belongs to the $a$ priori of physics?

To us ancient atomism appears to have offered an extremely promising alternative to the animistic conceptions widely accepted in antiquity. But atomism never "caught on"; it was, as Sambursky observes, fatally incompatible with the whole cosmology of the age.

Today we aim at projecting the mathematical and physical laws of the physical universe into man, with the object of explaining the phenomena of life by physics and mathematics; whereas the Greeks sought to extrapolate man into the expanse of the cosmos and regarded the cosmos as a living organism. Their biological metaphors, such as the breathing of the cosmos, are not simply allegorical: they really mean that the cosmos has its own rhythm of life, that its laws are basically organic and that therefore it is conscious of the musical harmony of the spheres. The conception of the world as a living body was present in all periods of Greek science. Any deviating tendency, such as the atomic theory, did not take firm root in the science of the Ancient World.

The linguistic factor. Almost a century ago, long before Sapir and Whorf reached their more extreme conclusions, Stallo already found it obvious

. . . that the thoughts of men at any particular period are limited and controlled by the forms of their expression, viz., by language (using this term in its most comprehensive sense); that the language spoken and "thought in" by a given generation is to a certain extent a record of the intellectual activity of preceding generations, and thus embodies and serves to perpetuate its errors as well as its truths; that this is the fact hinted at, if not accurately expressed, in the old observation according to which every distinct form or system of speech involves a distinct metaphysical theory; . . .

From that metaphysics we do not fully escape even as we acquire scientific and mathematical languages. Always in thought, if not also in statement, we supplement those languages with parts of everyday speech. Into science then stretches the aura of the covert cosmology enshrined in common-sense language: the existence of "things"; 
the dichotomies of subject and object, mind and body, wave and particle, etc.

Reflection of the logical sense of those who made it, a language cannot but condition the reasoning of those who use it, whether in science or elsewhere. A language conditions the way in which questions are put and the way in which answers are formulated; to some extent it determines even what questions can be put and the kinds of answers that can be given. As questions put to nature, even our experiments will not then escape conditioning by the cosmology of our language and, as Whorf strongly emphasizes, the same irreducible element of subjectivity will color what we like to think of as nature's answer.

. . . the world is presented in a kaleidoscopic flux of impressions which has to be organized by our minds-and this means largely by the linguistic systems in our minds.

. . . no individual is free to describe nature with absolute impartiality but is constrained to certain modes of interpretation even while he thinks himself most free.

. . . users of markedly different grammars are pointed by their grammars toward different types of observations and different evaluations of externally similar acts of observation, . . .

The aesthetic factor. "Taste" for classic simplicity or baroque complexity-or taste perhaps gratified in great and multiplex ends produced by few and fundamentally simple mechanisms-such taste shapes cosmology and, thence, enters into the making of "scientific taste." Dingle cites an amusing example of the aesthetic factor, significant though it was pronounced in the 13th century, well before the rise of modern science. Contemplating the extremely cumbersome Ptolemaic system, the devout and learned Alphonso of Castile was moved to remark that, had he been present at the Creation, he could have given the Creator some good advice. The same feeling of repugnance for the Ptolemaic system enters into the complex of motivations that stirred Copernicus to the creation of his system; and aesthetic appeal almost alone sustains Copernican astronomy through the difficult first half-century of its life.

Consider that the Copernican system could be, and was, held to "explain" the familiar only in terms of the preposterous. The changes in the heavens are "explained" by a triple motion of the earth of 
which we must suppose ourselves entirely unaware. The absence of stellar parallax is "explained" by the postulate-not merely arbitrary but actively repulsive-that the nearest stars are fantastically distant. Necessarily impugning scholastic interpretations of motion, the Copernican system could itself account for the continuance of celestial motions only by the suggestion of a special "naturalness" to motion in perfect circles. Inadequate at best, this "explanation" collapses with Kepler's demonstration that the planets do not pursue accurately circular courses. Why then did anybody credit the Copernican theory? Because of course to a few that theory offered a breathtaking vision of mathematical harmony. Polanyi well observes that we have here no simple rejection of the anthropocentricity implicit in a geocentric system but, rather, a preference for a different aesthetic anthropocentricity maintaining that nature is the embodiment of mathematical regularities harmonious in the ears of men.

A further element of aesthetic appeal sustained Copernicus' system in the face of the far sterner challenge posed by the Tychonic system for the next three-quarters of a century. Far more readily reconciled with scholastic mechanics, but mathematically the exact equivalent of the Copernican theory, Tycho's system remained clearly superior even as a correlative device until the work of Newton. The earth is stationary, and neither the "evidence of the senses" nor the absence of detectable parallax need then be explained away with additional postulates. We say that the Tychonic system is wrong, and rejoice that some men rejected it from the outset. On what basis could they possibly do so? As far as I can make out, the root of their rejection of the Tychonic system lay in its failure to assign the central position to that great luminary, the sun. To a Neoplatonic the sun "belonged" in the center, and he could cling to the Copernican theory simply out of regard for this element of aesthetic appeal wholly lacking in its competitor.

Aesthetic considerations remain powerful in science. Everlastingly compelling, considerations of symmetry (physical and/or mathematical) are no less so in the present era of quantum mechanics. Indeed, but a few years ago the concern for symmetry evoked from de Broglie a distinct echo of Copernicus' opinion that, to be acceptable, a theory must appear "sufficiently pleasing to the mind."

. . . some physicists have even come to doubt the existence of a real symmetry between light and matter concerning the duality of their 
nature. On this point we are of an exactly opposite opinion: the symmetry between matter and light, which served as the basis of the development of wave mechanics, is so satisfying to the mind and to us seems so much to be the profound reason for the success of these new theories, that, in our opinion, we must not abandon it at any price.

In scientific creation the activity of the aesthetic factor is often patent -if not in de Broglie's own work, for example, then surely in those considerations of "mathematical perfection and beauty" that, says Born, guided "Maxwell's decisive step" in the framing of his electromagnetic theory. In scientific judgment the activity of the aesthetic factor remains evident as long as we characterize the theories we accept as "beautiful," "elegant," and "refined," and those we reject as "clumsy." There is some justice even in the extreme view of du Noüy, who writes:

Whenever there is no objective confirmation, our attitude toward certain theories depends, in the last resort, on aesthetic considerations, disturbing as this may seem.

The eihical factor. A science need only be "true"; a cosmology we hope to find "good." In any society the powers that be (church, state, school, press, vox populi, etc.) will hold certain cosmologic views "good" and others "bad"-in that they impugn sound religious doctrine, good citizenship, the healthy outlook, etc. Thus, for example, to Plato the naturalistic view appeared a danger to the state:

The theories of our modern men of enlightenment must be held to account for the mischief they cause. Now the effect of their compositions is this: when you and I produce our evidence of the existence of gods and allege this very point-the deity or divinity of sun and moon, planets and earth-the converts of these sages will reply that they are but earth and stones, incapable of minding human conduct, however plausibly we have coated them over with a varnish of sugared eloquence.

To prevent the "mischief," Plato urged that teaching of the naturalistic doctrine be proscribed, and its teachers imprisoned. Apparently action of this sort never became a major factor in the life of ancient science. But as science assumes a larger role in cosmologic construction, the pressure "authority" brings to bear on the shaping of cosmology will be felt increasingly in science also. The flames that consume Bruno threaten to spread to Galileo: the attempt to suppress Copernican cosmologies becomes in time an attack on their essential foun- 
dation, Copernican astronomy. Some two and one-half centuries later, horror of the cosmologic overtones of an ethically blind natural selection produces the furious attack on Darwin's biology. In our own day the ethical factor prompted the Soviet regime to suppress one genetic theory (and to extirpate its supporters) in favor of another that was felt to be more readily compatible with the demands imposed by the state philosophy.

The scientist is not simply the passive victim of external pressures. Ethical considerations enter into the construction of his own cosmology-and thence into his science. To be sure, he is taught to do his science without regard to such considerations, but this is a counsel of perfection. Will a mechanism of natural selection first commend itself to a scientist who believes that natural phenomena express the development of some mighty ethical purpose? Was the judgment of Russian geneticists wholly unaffected by their acknowledgement of the official philosophy of the state? Einstein's passionate refusal to accept as final the statistical description, furnished by modern quantum mechanics, cries an anguished protest against the conception of nature governed by "a god who plays at dice." We smile condescendingly at Einstein's attempt to inject into scientific discussion what is transparently an ethical argument. Can we afford the condescension? Is not acceptance of amorality itself an ethical decision? When the quantum physicist most vigorously denies all cosmologic commitments and pretensions, has he not already given tacit assent to the Lucretian cosmology-with its blind, purposeless, random tumbling of particles in the void?

The moral factor. Only rather arbitrarily distinguished from the ethical factor, some moral elements in the climate of opinion act powerfully on science. Consider for example the effect of Calvinist emphasis not on God's love but on God's will: the doctrine of predestination is perhaps necessarily associated with a cosmologic conception of immutable law. A faith in the lawfulness of the universe is surely essential to science, and Needham well inquires:

Was the state of mind in which an egg-laying cock could be prosecuted at law necessary in a culture which should later have the property of producing a Kepler?

One cannot progress, or even think of progressing, in scientific understanding if he considers nature ruled by one or more capricious or 
even actively malevolent deities. Modern science takes its departure from a quite different moral conviction-kin perhaps to Einstein's faith that:

Raffiniert ist der Herr Gott, aber boshaft ist er nicht.

The Lord God is subtle, but malicious he is not.

Beyond belief that man can understand nature, there is the further feeling that man may be obligated to seek such understanding. His mind, the gift of God, is most fittingly employed in searching the world that God created for what Bacon calls that

spark of knowledge of God which may be had by the light of nature and the consideration of created things; and thus can be fairly held to be divine in respect to its object and natural in respect of its source of information.

This conception of natural religion becomes a powerful stimulus to science-particularly in England right through the period of Priestley -and perhaps even today it has not fully spent itself, for Oppenheimer notes that:

. . . Einstein has seen in his theories of relativity only a further confirmation of Spinoza's view that it is man's highest function to know and to understand the objective world and its laws.

Here surely we gain some sense of what Snow describes as "a moral component right in the grain of science itself."

Powerfully influenced by accepted religious doctrine, the scientifically relevant moral tone of a society depends also on other factors. Of four predominantly Roman Catholic countries-Italy, France, Spain, and Ireland-the first two have developed important indigenous scientific movements, while the last two have not, though each of these was at one time a substantial center of learning. Observe however that, unlike Spain and Ireland, France and Italy have sustained important movements of religious dissent. May a decisive factor be the extent to which dissent is accepted as a moral right? If not also elsewhere, in science capacity for dissent (from existing "self-evidencies") and the possibility of progress are firmly linked. If Roman Catholicism does not encourage capacity for dissent from established doctrine, can this explain the results of the Notre Dame 
studies showing the disproportionately small number of American Roman Catholics among important American scientists? ${ }^{*}$ Capacity for doubt and dissent may, on the other hand, abound in one who -as member of an "out-group" (perhaps a religious minority)-is forced to regard existing orthodoxies from the outside, and so more sceptically than the orthodox for whom orthodoxy is final. The predominance of dissenters in early English science, and the disproportionately large number of Jews in the ranks of major scientific innovators, perhaps finds here some explanation. Not all out-groups produce a multitude of great scientists. Insufficient in itself, scepticism must be complemented by a moral faith that, in the dissenting and Jewish traditions, judges scholarship a matter of "value."

\section{THE COSMOLOGY OF ORGANIZED SCIENCE}

Cosmology is not a highly organized enterprise: each scientist is his own cosmologist, and to each his own individual cosmology is, overwhelmingly, the most appealing. Multiple factors originating in his local community enter into the cosmology of each, and thence into his science. How then can science have unity? By virtue of its own existence as a social entity! Organized science creates and maintains its own cultural milieu, its own ideological atmosphere, in which the scientist dwells so long as he studies science and works as scientist. Organized science has its own language ("scientific language"), its own aesthetics ("simplicity"), its own ethics ("truth is the supreme good"), its own morality ("the search for truth is the supreme moral obligation"). To these add a set of principles, accepted by practically all scientists of a given generation, which determine the whole texture of scientific thought. Summing over all, one finds a respectable approximation to a corporate cosmology.

Nowhere stated as such, this cosmology forms part of the scientific tradition with which the individual scientist becomes familiar during his education, and in his later dealings with his colleagues. Encouraged by this tradition to disregard the various influences originating in the social milieu of his native community, the scientist can never wholly disregard them-but their effect is greatly attenuated or diluted by the cosmology of organized science. Despite the variations in

" On this very large subject see R. K. Merton, Social Theory and Social Structure (Free Press of Glencoe, 1957), chapter 18, "Puritanism, Pietism, and Science." 
individual cosmology, a substratum of identity in the cosmologies of all scientists makes possible the unity of scientific endeavor.

Consider also the indirect effect of science on itself: everywhere science modifies, and makes more uniform, the scientifically relevant climate of opinion. Our aesthetic sensibilities are deeply conditioned by the simple majesty of certain scientific theories incorporated in cosmology. Poincaré exclaims:

One may dream a harmonious world, but how far the real world will leave it behind! The greatest artists that ever lived, the Greeks, made their heavens; how shabby it is beside the true heavens, ours!

Our ethical sensibilities are not unaffected by the concept of an evolution controlled by blind natural selection produced in the struggle for existence. Not even our moral judgments are left wholly unchanged, for as Butterfield observes:

. . . the so-called "scientific revolution," popularly associated with the sixteenth and seventeenth centuries, ... outshines everything since the rise of Christianity and reduces the Renaissance and Reformation to the rank of mere episodes, mere internal displacements, within the system of medieval Christendom. Since it changed the character of man's habitual mental operations even in the conduct of the nonmaterial sciences, while transforming the whole diagram of the physical universe and the very texture of human life itself, it looms so large as the real origin both of the modern world and of the modern mentality that our customary periodisation of European history has become an anachronism and an encumbrance.

\section{Technology}

Like science itself, technology comprises both activity (of engineers, technologists, practicing physicians, etc.) and the fruits of the activity (factories, goods, and services). Living as we do in a world of scientific technology, we find it almost incredible that until quite recently technology should have led the way-with science following, rather ineffectually, in its train. But consider the situation in, say, prescientific iron metallurgy. The metallurgist knew that, to make iron, one must heat its ore with charcoal under certain conditions. In the group of colligative relations defining this and other operations of his craft, the metallurgist held prescriptions for success which science was for long wholly unable to improve. 
As chemistry opened out at the beginning of the 18th century, the metallurgists' processes were still a challenge to chemists, whose studies ultimately put them in position to offer the working metallurgist a rationalization of his practice: ore is made metal as phlogiston passes into the ore from the charcoal. But this rationalization suggests no improvement in metallurgical practice, and had little if any effect on that practice. Toward the end of the century Lavoisier created his new oxygen theory, offering a new rationalization: ore is made metal as oxygen is taken out of the ore by the charcoal. But again the working metallurgist is no better off for the explanation. The impact of Lavoisier's innovation in chemistry is not felt in the metallurgical industry until long after Lavoisier's death.

Today the situation is dramatically different, in metallurgy and elsewhere. Binkley seeks to pinpoint the time at which science first became a major factor in technology:

Comment on the Great Exhibition [London, 1851] usually linked mechanical arts and science, but not in the sense that science was the leader and art the follower. Whewell, the historian of science, . . . took it for granted that the natural and "proper sequence" was for creative activity in the arts to go first, and science to follow after with its speculations-exactly the process that was taking place in the development of the doctrine of thermodynamics from the steam engine. In 1867, when Paris held its second World Exhibition, the place of science in its relations to industry was noticeably changing. By that time the aniline dyes had arrived as the products of organic chemistry, and a number of electric dynamos were on exhibit. When Michel Chevalier, the free-trade economist, wrote his introduction to the jury reports of the Exhibition, he attributed increase in productive power to the advance of science. . . . the difference between Whewell's attitude in 1851 and Chevalier's in 1867 can be taken as marking the point at which science established before the public a claim to the leadership in the industrial arts. . . .

Probably the transition is not quite so abrupt as Binkley suggests. Even a century earlier than this science had already contributed actively to technology-improved navigational aids, a preparation of sulfuric acid (fundamentally the alchemists') operable on an industrial scale, and so on. From the middle of the 18th to the middle of the 19th century the applied arts derive from science an ever increasing number of small but crucially important contributions, e.g., chlo- 
rine as a bleaching agent, and the miner's safety lamp. However, Binkley is quite right in maintaining that only about a century ago did the actively creative role of science in technology become apparent-with the establishment of electrical and chemical industries that owed their creation wholly to the antecedent existence of science. From the thus completed interaction between them develops a genuinely symbiotic relation of science and technology.

Conceptual exchanges. The "gas laws," relations worked out entirely in science, render important services to technologists concerned with the design of compressors, superchargers, internal combustion engines, steam and gas turbines, and the like. In place of the many separate relations, the engineer may prefer to use the correlative device constituted by the kinetic theory: he works then with a few more general equations in which all the individual laws are implicit. In either case, by routine use of the anticipatory apparatus, he avoids wasteful cut-and-try endeavors; he more swiftly attains his goals by processes having, in Conant's phrase, a "low degree of empiricism."

For all its progressiveness, technology tends to maintain itself within a fixed frame of reference, by which ultimately it can be confined. A spectacular liberation may then occur under the impact of new ideas generated in science. Consider rapid communication as the problem it was in 1800 . Treating it purely technologically, one might seek to achieve brighter beacons (or larger mirrors) on higher hills, smoother post roads traversed by horses bred for speed and stamina, the use of homing pigeons similarly bred, and so on. Were one well traveled, he might even think of trying larger drums or blacker smoke. Exploitation of such possibilities does indeed lead to technologic advance, which is however inevitably canalized and limited by a narrow conception of what are possible means of communication.

In 1800 no technologist ignorant of science could apprehend the reformulation of the problem of communication that was to be produced from the bizarre studies-of the attraction of bits of chaff to rubbed amber or glass, or of the twitching of the leg of a dead frog -which fascinated a few contemporary scholars. Yet, well within half a century, these studies eventuated in scientific ideas that formed the basis for the development by Morse and Bell of the telegraph and the telephone, respectively. These were revolutionary approaches to the problem of communication. The same cycle is subse- 
quently repeated. Great advances in telegraphy and telephony, conceirable in 1850, were achieved as (with the aid of a few scientists like Lord Kelvin) technology set out to explore to their ends the ruts newly formed in the problem of communication. What could not be foreseen in 1850 was that Faraday's physical concept of "field" would shortly lead to Maxwell's subtle electromagnetic theory, and that in turn to certain experiments by Hertz. A few years after the turn of the century Marconi's wireless signals would span the Atlantic. But without the antecedent Faraday, Maxwell, and Hertz there would be no Marconi. Science breaks for technology trails not merely new but previously inconceivable.

Technology makes reciprocal conceptual contributions to science. Today science has its own dynamism, generating internally a large proportion of the problems, the excitement, the new colligative relations, and the analogic stock for which it once drew heavily on external sources like technology. Even today, however, technology remains an important contributor of all, and not least important for its supply of analogic stock. Earlier in this century the functioning of the brain was conceived with the aid of the analogy furnished by the telephone exchange. More recently cybernetics finds what appears a more appropriate analogy, in electric networks with many negativefeedback loops. "Feedback" is itself a concept of technologic originfirst exemplified in the Watt engine-governor and, more recently and abundantly, in electric circuitry.

Material exchanges. Obvious, and obviously important, these material exchanges will be no more than indicated. By supplying the requisite elaborate glassware, the glass factory at Rouen played an essential role in Pascal's enlightening variations of Torricelli's experiment. The balances that served Lavoisier and Stas were fabricated by the makers of balances for government mints. As the tools of science become ever more complex, science becomes ever more dependent on the technological resources it can tap. Influencing the ways scientists attack their problems, this dependence may determine even what problems can be attacked.

A reciprocal flow of materials, tools, and techniques passes from science to technology. Nowhere available outside the scientific laboratory, the rare and expensive substance aniline is found by Perkin to yield a valuable dye, mauve. To obtain mauve Perkin institutes a wholly novel large-scale production, from coal tar, of aniline-which 
becomes in consequence a cheap and abundant starting material for chemical inquiries previously impossible. In such exchanges the symbiosis of science and technology finds its clearest expression. Hertz's short-range transmitters and receivers are, through technologic development, transmuted into efficient radio equipment. This, in the hands of scientists, leads to further explorations in which the possibility of radar detection is first manifested. A further technologic development yields practical radar devices, the newly available components of which open to exploration new areas of fundamental scientific research, e.g., microwave spectroscopy.

\section{SCIENCE AND TECHNOLOGY}

Their intimate symbiotic relation makes difficult a satisfactory distinction of science from technology. Define science as what scientists do? But men like Perkin, Caro, Kelvin, and Pasteur have shuttled back and forth between what is clearly science and what is no less clearly technology. Define science rather in terms of results that combine novelty with generality? A much better possibility, I think. Only novelty that has some generality is scientifically interesting novelty, and Poincaré remarks:

... scientists believe there is a hierarchy of facts . . . the most interesting facts are those which may serve many times; these are the facts which have a chance of coming up again.

What then is a good experiment? It is that which informs us of something besides an isolated fact; it is that which enables us to foresee, that is, that which enables us to generalize.

Consider an example. While seeking means to reduce or eliminate internal blackening in the incandescent lamp, Edison stumbled on what we call the Edison effect. This remained an isolated curiosity for some years, but ultimately was caught up in the web of the theory of the electron elaborated by J. J. Thomson. Thus identified with the emission of thermo-electrons, the Edison effect becomes a crucial factor in such further advances as the Fleming valve and the de Forest triode-early harbingers of a flood of electronic devices of immense importance to both technology and science. The Edison effect is science: the discovery of a new and general phenomenon. Edison's far more celebrated invention of the incandescent lamp is not science. Exploiting well known general relations-e.g., a current heats a resistor, a body strongly heated becomes incandescent, no 
combustion takes place in a vacuum, etc.-Edison drew from his own systematic empiricism the discovery of a suitable filament, and from his great ingenuity the means of putting together a practical device. His product is a genuine novelty, a new "fact" if you will, but not a fact of interest to science. Here is the root of the disdain with which scientists not infrequently regard inventions (and inventors). For example, Bell's invention of the telephone draws from Maxwell this comment:

When at last this little instrument appeared, consisting, as it does, of parts every one of which is familiar to us, and capable of being put together by an amateur, the disappointment arising from its humble appearance was only partially relieved on finding that it was really able to talk.

Undeniably a new and important "fact," the telephone is scorned by Maxwell because the general elements on which it depends are not novel, and the element of novelty that it represents is not generalizable. Precisely the same dipartite inadequacy disqualifies the invention of the incandescent lamp as science.

With the results in hand, the distinction of what is science from what is technology may be feasible; but a distinction drawn after the event does not fully satisfy us. Can we identify in advance those inquiries likely to yield results of interest and value to science? At one time the intent with which work was instituted offered some basis for prognosis. The effort to develop a practically valuable product or process could then be classed as "technology"-unlikely to produce results that duly combine both novelty and generality. Attention to the site of the activity often rendered prognosis even more secure: from a university laboratory one expected "science"; from an industrial laboratory, "technology." Today both criteria have become seriously inadequate. As to site, a few industrial laboratories now have an appreciable output of "science" at its best: the diffraction of electrons, a new class of semi-conductors, and a new foundation for communication theory are all discoveries emanating from the Bell Laboratories, for example. On the other hand, some academic laboratories now have as their chief product work that is, at best, "technology." As to intent, in research on cell metabolism the scientist (and his financial backers) can never be wholly insensible of the investigation's possible bearing on the problem of cancer. In such areas 
there can no more be a perfectly "pure" science than, as Picasso remarks, there can be a perfectly abstract art:

There is no abstract art. You must always start with something. Afterwards you can remove all traces of reality. There's no danger then anyway, because the idea of the object will have left an indelible mark. It is what started the artist off, excited his ideas, and stirred up his emotions.

Constraint. Perhaps the least unreliable forecast of the scientific importance of any given inquiry turns less on the nature of the investigation than on the talent of the investigator: he who has already discovered much is not unlikely to discover more. But forced to wear blinders, even the gifted scientist sees less. Priestley's comment on his own career applies also to many other distinguished scientific careers:

In looking for one thing I have generally found another, and sometimes a thing of much more value than that which I was in quest of.

Other things being equal, more will be discovered when the investigator retains power to pursue promising tangential leads opening up during his investigation. A Rayleigh, prosecuting a self-assigned investigation, is free to follow his research wherever it leads him. Turning aside from his initial study, he traces a curious anomaly back to its root, and so discovers a new element, argon. But a Hillebrand, working toward a specified goal in a government laboratory, could not stop to examine a similar anomaly which pointed to a similar discovery: the terrestrial occurrence of the element helium. In a retrospective reflection, Hillebrand wrote that

. . . the chemical investigation had consumed a vast amount of time, and I felt strong scruples about taking more from regular routine work.

For this and other reasons he failed to look into

the suggestion made by one of us in a doubtfully serious spirit, that a new element might be in question.

The successes won by Rayleighs and lost by Hillebrands suggest to Conant that the presence or absence of constraint is a distinction far more important than that now doubtfully drawn between pure and applied science. One might attempt to distinguish as applied 
science the endeavor to prepare a nitrogen mustard with superior properties as a chemotherapeutic agent and as pure science some general study of cell metabolism. The second project is perhaps more likely to yield results of interest to science, but either may do so and neither may do so. The first project is more likely to proceed under constraint, but in both cases constraint to a rigid program set down at the outset sharply reduces the chances that scientifically important results will be obtained. Trotter properly emphasizes that:

Great discoveries will . . . continue to be unexpected and the advance of science occur on an irregular front in which the salients mark the places where amongst the facts the going is good. Even research deliberately directed against short-range targets is apt to be held up contrary to all reasonable expectation or to score its successes through unintentional deflections.

Emphasis on science immediately applicable to the problems of technology necessarily diverts support, and men, from the kind of science that-following to their unexpected ends lines of research having little or no apparent relevance to current technologic problems and practice-leads ultimately to complete reconstruction of the technologic horizon. The utterly unprecedented dynamism today displayed by science and technology is founded on the fully developed symbiotic interaction between them. This interaction ensures that any loss to science will be a loss felt also by technology, and the dynamism ensures only that the loss will be felt sooner rather than later. An understandable concern for national security may suggest the desirability of secrecy imposed to protect the scientific discovery made the year before last, or constraint imposed to secure the fullest possible exploitation of last year's technologic triumph. But in the present dynamic situation these policies are completely self-defeating if as a result science does not this year arrive at the basis of what will become next year's technology. Even the "purest" scientist is not unmindful of the possibility of such advances. If, as science develops under its own internal dynamics, he can grasp the possibility of some previously impossible resolution of an urgent technologic need or problem-then there await him fame and money; beyond such "sordid gain," perhaps the satisfaction of the benefactor of his community; and always the prospect that, through technologic exploitation of his science, his materials and tools will be returned to him a 
hundredfold improved and multiplied. There is then some automatic co-ordination of scientific endeavor and technologic needs, co-ordination achieved without any of the losses entailed when constraint becomes a general policy for science.

\section{THE REPROBATION OF SCIENCE}

For better or worse, the automobile affects social mores; radio, television, and the cinema condition aesthetic taste. Acting through technology, science changes the whole pattern of our culture. Our central-city civilization can first arise only with the development of artificial fertilizers that vastly increase the yield of agriculture, public health measures typically reflected in municipal water systems, and so forth. Today we are most acutely aware of some rather different technologic exploitations of science. Contemplating the nuclear weapon and its intercontinental carrier, we may well feel stirred to moral rejection of the science that has made them possible.

Science vastly multiplies and diversifies the range of possibilities humanly attainable, among which we choose those we will make realities. But unless we reject as morally evil the ultimate scientific commitment to the quest for knowledge-and some of course domoral condemnation of science is nonsense. The scientist makes an ethical judgment, and assumes a moral responsibility, when he elects to take part in the technologic exploitation of science for destructive purposes. "Social demand" may applaud but cannot itself justify such a decision-any more than it can the decision of the smith who turns iron into swords rather than plowshares. But scientific knowledge is ethically as neutral as iron: "evil" only when forged as a sword, "good" when beaten into a plowshare. Conceivably there is some knowledge that can lead only to "evil"; certainly there is no knowledge that can lead only to "good." The discovery of some marvelous vaccine against a lethal plague would seem solely "good"; but it opens up also wholly new possibilities for "evil"-an aggressor will wage germ warfare only if he possesses means to protect his own population. Science a curse, its exploitation bringing death and disaster? It may be so. But life is commonly esteemed the supreme blessing; and the vast majority of us would not now be alive save for advances in agriculture and hygiene that are also exploitations of science. Ambivalence attaches to the works of science simply because their technologic exploitations rest in the hands of men. 


\section{THE EFFICIENCY OF COLLIGATIVE RELATIONS}

In Chapter II we observed that from a typical colligative relation like the law of the lever we obtain predictions that may prove only approximately correct. Consider now how I will proceed when I seek to draw from the law of the lever predictions exact to the limit of my capacity to check them experimentally. Obviously I do well to begin by checking the graduation of my meter stick and the calibration of my weights; but I can hardly expect reliably to predict the behavior of a system incompletely defined in any "significant" sense. I must then carry through an extensive study, beginning perhaps with examination of the external conditions. Ideally the experiment should be made in a room or enclosure free from drafts (which might disturb the condition of balance), held at a uniform constant temperature (to avoid any uneven expansions or contractions of the beam); constant humidity ( to avoid the adsorption or desorption of water on or from the beam or the weights), and constant pressure (to avoid variable buoyancy effects on beam and weights that have finite volumes ). Lacking these ideal conditions, I can still proceed, by calculation from measurements of the external conditions, provided that I have amassed much information about the components of the system: their volumes, surface areas and characteristics, coefficients of thermal expansion, and so on.

About the actual system I will in any case need very precise information. Are all the bearing surfaces essentially frictionless, coplanar, parallel to each other, and perpendicular to the beam? If not, further measurements and calculations must supply appropriate corrections for the deviations. What is the coefficient defining the rigidity of the beam, what does it weigh, and where is its center of gravity? This last I cannot calculate from the geometry of the beam without making the potentially fallible assumption that it is perfectly homogeneous. Presumably I will prefer to make a preliminary trial to determine the position of the fulcrum when the unloaded beam is in balance; but this, rather painfully, necessitates that I assume at least some part of the law of the lever in deriving the predictions by which I hope to establish its exactitude.

Completing all these preliminary studies, I can make predictions I would be vastly surprised to find detectably in error. Nevertheless the possibility of at least minor error always exists and occasionally 
materializes: some of the preliminary measurements may have been faulty, some of the many variables I thought it safe to ignore may actually have affected the outcome of the test, etc. I can still argue that the law of the lever is absolute, exact, and "true" as applied to the hypothetical ideal lever: by definition that retreat position is always secure. But as a colligative relation usefully applicable to actual lever systems the law is clearly contingent (since prediction depends on potentially fallible antecedent determinations of conditions), approximate (since all the possibly relevant conditions cannot be controlled or determined), and at most efficient (to the extent that it represents an optimal reconciliation of our conflicting desires for ease and generality of application and for reliability ).

That our desires conflict is nowhere clearer than in the last example. As ordinarily conceived, the law of the lever is a relation of great generality. But, seeking to draw from it predictions of maximum reliability, we had sharply to shrink its applicability. In effect, we limited it to systems approximating an analytical balance under the closely controlled conditions of the laboratory-to systems approximating in their extreme artificiality the extreme abstraction of the ideal lever. Meyerson writes:

Doubtless, if nature were not ordered, if it did not present us with similar objects, capable of furnishing generalized concepts, we could not formulate laws . .

In fact, we only attain laws by violating nature, by isolating more or less artificially a phenomenon from the whole, by checking those influences which would have falsified the observation. Thus the law cannot directly express reality. The phenomenon as it is envisaged by it, the "pure" phenomenon, is rarely observed without our intervention, and even with this it remains imperfect, disturbed by accessory phenomena.

The artificiality of the special lever system with which we worked reflects our attempt to isolate it from the rest of the universe, to insulate it from the manifold accessory factors operative in the raw phenomenon but omitted in the statement of a law that refers explicitly only to certain weights and distances. Ordinarily sufficient (though not perfect) isolation, insulation, can be achieved: that is the message of the principle of dissolubility. And ordinarily we arrive at acceptable predictions without taking account of more than a very few complicating factors. We do not, cannot ordinarily, even con- 
template undertaking the extravagant series of studies essential for exact prediction. Demanding such studies, the law of the lever would lose all its value: with but a fraction of the effort required for prediction we could simply set up the lever system and observe what happens. Thus, as Duhem remarks,

A mathematical deduction . . . may therefore be useful or otiose, according to whether or not it permits us to derive a practically definite prediction of the result of an experiment whose conditions are practically given.

Predictions drawn with difficulty from the more complicated van der Waals relation are usually more reliable than those drawn from Boyle's law. But ordinarily we elect to use Boyle's law-from which we easily derive a host of predictions usually quite well enough borne out in practice.

Paraphrasing Einstein, may I not insist that insofar as a colligative relation is generally applicable it is not exact, and insofar as it is exact it is not generally applicable? What we seek in a colligative relation is efficiency-a reasonable balance of reliability, generality, and convenience-and efficiency is all that is attested by the successful functioning of a colligative relation as such. To be sure, in their cosmologic speculations, scientists have sometimes claimed discovery. of the true laws by which nature is governed. That cosmologic dogma is, however, no present concern of ours-as becomes clear when we consider, for example, the equivalence of inertial and gravitational masses. We may hold it universally and absolutely "true" because we accept as final the relativistic theory in which this equivalence is $a$ deduction entailed by postulates we accept. But then surely we are not treating it simply as a colligative relation. Alternatively, we might hold the equivalence "true" because it has been confirmed to the limit of our experimental measurements. Here we introduce the covert assumption that a law so confirmed must be mathematically exact, and so unite the colligative relation with a superfluous metaphysical assumption. As a colligative relation the "law" is no more than efficient. The generality of this important conclusion is perhaps best illustrated by showing its applicability to a colligative relation apparently wholly different in form from any so far examined.

The sulfur relation. Sulfur is a yellow crystalline solid with density 2.07, melting at $119.0^{\circ} \mathrm{C}$ and boiling at $444.6^{\circ} \mathrm{C}$, a good insulator, 
soluble in carbon disulfide, burning in oxygen to give a gas . . . and so on. Is this any more than a definition of what we mean by "sulfur"? Meyerson teaches us to see that the definition yields the following perfectly genuine colligative relation: If a substance displays many of the following properties ... . then probably it will display all the others. Such a relation offers multiple predictions of which many have been confirmed repeatedly. Can any such prediction ever fail? Yes! The definition notwithstanding, every chemist well knows that the sulfur of experience may be quite different from the ideal sulfur of the definition. "Real" sulfur may be not yellow but white; not hard and crystalline but rubbery or amorphous; melting not at $119.0^{\circ} \mathrm{C}$ but at some other temperature dependent on its previous history, purity, and isotopic composition-and so on.

What to do? Restrict application of the relation to some standard sulfur prepared by a specified procedure? Certainly not! Just as we guard the generality of such concepts as "water" and "lever," we insist on keeping "sulfur," and its defining relations, conveniently applicable to the myriad specimens of real sulfur-each with its own impurities and its own history. Seeking efficiency, we make the characteristic compromise, as is perhaps most evident when we ask: How "many" properties must we check before identifying a substance as sulfur and predicting its other properties? Obviously, the more properties we check the less likely we are to make false identifications and false predictions. Ordinarily, however, we are content to check only one or a few properties-perhaps even none beyond the label on the container.

\section{A COLLIGATIVE RELATION: BOTH INVENTION AND DISCOVERY}

We invent the concepts weight and distance; we invent the ideal lever to which the law of the lever applies by definition. But the law acquires value only through a simultaneous discovery: there are in nature distinguishable systems predictably conformable to the law. Similarly, the concept sulfur is an invention, scientifically meaningful only because we often encounter recognizable specimens sufficiently approximating "ideal" sulfur that useful predictions can be drawn from the sulfur relation. These complementary elements of invention and discovery are evident even in the more abstract scientific concepts. Some writers dispute whether Thomson invented or discovered the electron. This futile issue is easily resolved: the electron 
is both invention and discovery. Purely as a concept it is an invention; but its scientific value becomes established only with the discovery in our experience of certain items readily interpretable in terms of this concept.

Boyle's law. In this new context let us examine in greater detail the complex passage, from observational data to scientific law, that was only sketched when the law of the lever was discussed in Chapter II. Imagine that we invent a J-tube apparatus, like Boyle's own, with a pocket of air trapped by mercury in the closed short limb. We observe
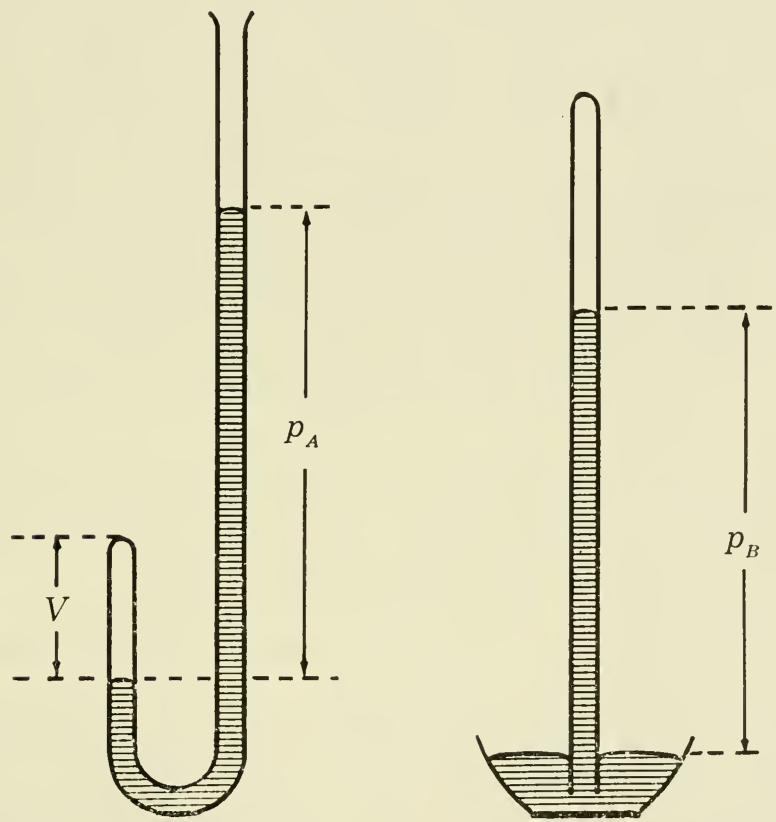

At left: A small volume of air, trapped in the closed arm of the J-tube, is compressed into a smaller volume when more mercury is added. The total pressure acting on the confined gas is made up of the hydrostatic pressure, $p_{A}$, plus the atmospheric pressure, $p_{B}$, acting on the mercury surface in the open arm of the J-tube.

At right: A barometer permits the determination of the atmospheric pressure which, acting on the surface of the mercury in the dish, holds the mercury in the evacuated tube at a height that reflects the magnitude of the atmospheric pressure, $p_{B}$. 
that, as more mercury is poured into the open end of the longer limb, the mercury levels ascend in both tubes. Paying no attention to the complicated (and irreproducible) oscillations of the mercury columns, we note their positions only after they have become quiescent. This is our choice, but to it corresponds something that is our discovery: the final positions of the mercury columns are reproducible and apparently independent of the antecedent oscillations.

That the two columns ascend together tells us essentially nothing. Indeed, we must not be bothered with the increasing length of the mercury column in the short limb: we note instead the decreasing length of the column of air therein. This length we conceive a measure of the "volume" of the entrapped air. And, similarly, we must not measure the length of the mercury column in the long limb but rather the difference in the heights of the columns in the two tubes. To this difference we add the height of the mercury column, measured from the mercury level in the reservoir, in an entirely separate device-a Torricellian barometer. This complex procedure is directed by the subtle concept "pressure." Thus we translate certain observables into conceptual terms we invent, and this is no simple matter. Having made his experiments, Boyle did not himself arrive at the law that bears his name; his friend Townley had to point out to him that the product of pressures and volumes is very nearly a constant. And here, after all the many inventions, we reach a discovery -though not yet a discovery of Boyle's law as such.

Experiment furnishes a finite series of points on, say, a plot of $p V$ versus $p$; but Boyle's law represents a line on that plot. Relying on the principle of continuity, we link up the points with a smooth lineassuming nonexistent any discontinuities that may happen to fall between even close-spaced points. We close the gaps by a veritable generalization: we interpolate, on the strength of Newton's faith, and ours, that Natura non saltus facit. And, from the line thus in part invented, we read off any of an infinitely large set of values interpolated, with a confidence not much inferior to that we have in the comparatively small set of values measured. But still we have not attained to Boyle's law which, on our plot, is the equation of a straight line. Lamentably enough, no straight line does pass through all the experimental points.

With a gas space more constant in bore, a meter stick more carefully graduated, and so forth, the divergence of the points from a 
straight line may be reduced, but not eliminated. We look then for sources of "experimental error." Perfectly typically, we find them in variables originally passed over as "irrelevant." We find the points very much less scattered when we control the temperature with a thermostat. A brief wait ("to establish equilibrium") before each reading sometimes helps; otherwise the gas may remain slightly overheated by the compression consequent to rapid addition of the mercury. We find further that the gas sample must involve no equilibrium mixture (lest the number of gaseous particles change with the pressure), that it must be reasonably far from its critical point and also reasonably dry (lest at high pressure part of the sample be "lost" though condensation), that the two arms of the J-tube must be reasonably equal in diameter (lest there be "capillarity effects"), and on and on.

We find that the more we take such precautions the more we reduce the scatter of the experimental points. But always, in any large set of measurements, we will have to dismiss (perhaps as "reading errors") one or a few points that fall far from the line. And always, as Poincaré observes, the bulk of the points will still show residual scattering away from the line we actually draw.

. . . the curve that we shall trace will pass between the observed points and near these points; it will not pass through these points themselves. Thus one does not restrict himself to generalizing the experiments, but corrects them; and the physicist who should try to abstain from these corrections and really be content with the bare experiment, would be forced to enunciate some very strange laws.

If the points form an approximately rectilinear array, we draw the straight line, defined perhaps by the method of least squares, that goes nearest to but not through all of them. Not to take as "best" the curve that is the smoothest reasonable approximation to the experimental points-that restraint our scientific taste, long molded by a conception of gradualism, would find "strange" indeed. The slightly uneven line actually defined by the points is, we feel, uneven only because of small but always finite experimental errors. By an act of faith we then pass to the limit: we say that if there were no such errors there would be no deviation from exact linearity. We then write the mathematical equation: $p V=$ constant; but this expression refers rigorously only to the outcome of experiments we have not 
done and cannot do. Human invention plays here no inconsiderable role.

We are still far from Boyle's law as it is commonly conceived. We have made a few experiments, at a particular time and place, with a particular apparatus containing a particular gas. We aspire to far greater generality. We extend Boyle's law to measurements made by very different means, e.g., with pressures imposed by a solid piston. To the concepts pressure and volume we thus attach additional denotations we deem "equivalent." We assign the denotations; but also we discover, applying them to experiments, that in each case the product $p V$ is gratifyingly constant. Now, with a few additional trials, and further invocation of the principle of continuity, we generalize the law to apply in all places, at all times, and under all conditions of constant temperature. Drawing still further on the principle of continuity, we suppose that in nature there are not simply things but things of a species: on the basis of a few more trials we generalize our results to all "gases"-including varieties yet unknown -and so arrive at last at Boyle's law in all its lustrous majesty. The luster is of course somewhat tarnished by the subsequent discovery that Boyle's law applies exactly only to an "ideal gas" we must ourselves invent.

Here is a recapitulation of the steps by which we reached Boyle's law:

1. Creation of certain conceptual and experimental tools

2. Experiments of which "relevant" elements are reported in conceptual terms and "irrelevancies" are dismissed

3. Reintroduction of variables originally conceived "irrelevant," as restrictions on applicability of law

4. Rejection of deviant points, and scattering of points, as "experimental error"

5. Generalization of points to line: statement of law as a mathematical relation

6. Generalization to other systems, and to entirely different kinds of systems

7. Generalization of law in place, time, and circumstances

8. Generalization of law for all "gases"

9. Conception of ideal gas to which law applies rigorously by definition 
Rarely would one find in practice any such systematic sequence of explicit steps. Ordinarily these analyses are conducted unreflectingly, with implicit assumptions. But, however they are made, all these steps must be taken before Boyle's law can be pronounced. We may then wish to reconsider the major discontinuity, in the sequence fact-law-theory, that is commonly alleged to fall between law ("a description") and theory ("an explanation"). Belief in any such discontinuity founders when, reviewing the progression leading up to Boyle's law, one recognizes the extreme difficulty (if not utter impossibility) of detecting where observation ends and theoretical manipulation begins-where discovered fact gives way to invented abstraction. And we may indeed say of scientific laws generally what Royce has said of scientific theories-that they are

. . . neither unbiased reports of the actual constitution of an external reality, nor yet arbitrary constructions of fancy. . . . They are constructions molded, but not predetermined in their details, by experience. We report facts; we let the facts speak; but we, as we investigate, in the popular phrase, "taik back" to the facts. We interpret as well as report.

\section{DENOTATIONS: IF INDIRECT, YET SOLIDLY ESTABLISHED}

For its functions as a predictive device, a colligative relation must, we saw earlier, involve only concepts having reasonably clean-cut denotations. Let us now assure ourselves that the situation remains unaltered even when the concepts are notably abstract, and their denotations are established only indirectly, with the aid of an elaborate computation apparently irreducibly "theoretical." Consider, as a representative example, a combined form of Kepler's first and second laws: a planet traverses an elliptic orbit, with the sun at one focus, at such a rate that the radial line sweeps out equal areas in equal times. We readily understand that a "planet" is a presumptive "body" seen as a point of light that moves, more or less irregularly, against the background of the "fixed stars." But now what are we to take as the denotations of "sun-focused elliptic orbits," etc.? A more pedestrian case will point the way.

Grasping the appropriate conceptual denotations, we all combine certain observable terms in certain ways whenever we measure a "pressure." Just so with "sun-focused elliptic orbits." On the basis of various astronomical observations, we plot on paper an orbit for the 
earth. Everybody who attaches the same denotation to the concept of Keplerian orbit-that is, everybody who in this sense speaks the same language-will arrive at the same orbit. As with pressure, the orbit is computed in an agreed-upon way from observations on which all agree, too. Continuing in the same fashion, by a determinate set of mathematical manipulations we pass from observations of the positions of the planet in the sky to an orbit for it plotted on paper-much as we proceed from observations of a J-tube to a plot of $p V$ versus $p$. And now to prediction! Given Boyle's law and the plot of $p V$ versus $p$ we readily derive predictions of what we should (and ordinarily do) observe under specified conditions. Just so, given Kepler's laws and the plotted orbits, the inverse of the procedure by which past observations were rendered as "points" on paper yields us predictions of where in the sky a given planet will be observable at specified times in the future. These predictions are generally found quite respectably (though not perfectly) sound.

Functioning as a typical colligative relation, the Keplerian laws seem to differ from the other relations so far considered only in the magnitude of the paper-and-pencil operation which intervenes between observations made and observations predicted. But this magnitude may be no more than an incidental reflection of the state of scientific development. Thus years ago the derivation of densities (from observed weights and volumes), speeds (from observed times and distances), and pressures (from the observed heights of mercury columns) demanded paper-and-pencil operations today wholly eliminated by the use of such devices as the hydrometer, speedometer, and Bourdon gage. From such devices we can read "density," "speed," and "pressure" directly, and can more directly predict what will so be read. With primitive instrumentation an elaborate computational operation may be required; with more elaborate instrumentation, on the other hand, little or no such operation may be needed even to use Kepler's laws. Provided the specifications of the paperand-pencil operation are clearly understood and generally acknowledged-as they are in the case of Kepler's laws-the conceptual denotations remain firmly established in the one case as in the other. And by such anchoring colligative relations are secured from what might otherwise prove an irresistible drift into the status of conventions.

Each and every colligative relation, sooner or later, is challenged by some datum or data at variance with it. Lest science dissolve into 
chaos we must, Ste.-Claire Deville enjoins, rally to defend the threatened relation:

Every time an exceptional fact has been discovered the first task, even the first duty, practically imposed on the man of science has been to make every effort to cause the fact to come under the common rule by means of an explanation which sometimes requires more work and reflection than the discovery itself.

For the preservation of the rule we have abundant resources, as is shown in Chapter IX. Even if all these fail, we can almost always find an escape hatch in the assumption that the system in question is "nonideal." Thus, for example, we suppose that Kepler's laws fail of perfect accuracy because the motion of any given planet is "perturbed" by its interaction with other planets. And blithely we hypothesize ideal systems in which no such complications exist, and to which the ideal law applies perfectly. How then can we ever bring ourselves to recognize as genuine the failure $(\mathrm{s})$ of a colligative relation in which we have confidence? If the colligative relation is made true, regardless of what the data indicate, is it not simply a convention? Consider a relation that, at least superficially, well lends itself to such interpretation.

Galileo's law of free fall. We express this law mathematically as: $s=\frac{1}{2} g t^{2}$. Here $g$ is a proportionality constant that measurably depends only on the locale of the experiment, and $t$ is the "elapsed time" during which a "freely falling body," starting from rest, traverses the "vertical distance" $s$. The denotations of $s$, g, and $t$ seem adequately clear; but on many occasions the law may seem to fail, and fail badly. A sheet of paper or a feather do not fall in accordance with the law. A dead pigeon or a steel ball bearing, falling a short distance in air, conform to the law; but a live pigeon in air, or a steel ball bearing in oil, do not. Stipulate that a "freely falling body" can only be one falling in a vacuum, unopposed by viscous resistance? So doing we at once eliminate the discrepant cases just noted, but not all discrepant cases: even in vacuum charged objects moving in electromagnetic fields may not behave as "freely falling bodies."

How then do we treat the law? Quite simply. We assert that it is invariably valid in the absence of "extraneous forces." When the relation is found to apply satisfactorily we declare that we have dealt with a "freely falling body." When it does not so apply we declare 
that, due to the action of some "extraneous force," the fall has not been "free." Thus safeguarded from all possibility of failure, the law is then nothing but a convention? Nonsense! We lack absolutely definitive criteria for the recognition in advance of a perfectly freely falling body-just as we do for an ideal lever or pure sulfur-but we do not lack all criteria. We need not then wait until after the fact to rush in with postulated extraneous forces that explain away cases to which Galileo's law should not have been applied in the first place. Instead, as Poincaré observes, generally we can recognize, in the world of experience, those real systems sufficiently approximating the ideal systems to which the law applies by definition.

It would do me no good to have given the name of free fall to falls which happen in conformity with Galileo's law, if I did not know that elsewhere, in such circumstances, the fall will be probably free or approximately free. That then is a law which may be true or false [read "efficient or inefficient"], but which does not reduce to a convention.

Because we grasp the denotation of "freely falling body" the law is for us no "mere convention": by it we are invested with power to predict what will be observed, and almost always (though not invariably or precisely) this is just what is observed. Thus, substituting "relation" for Poincarés first ambiguous use of "fact," we may say with him:

all the scientist creates in a fact is the language in which he enunciates it. If he predicts a fact, he will employ this language, and for all those who can speak and understand it, his prediction is free from ambiguity. Moreover, this prediction once made, it evidently does not depend upon him whether it is fulfilled or not.

Might one not still object that some colligative relations are so deeply and generally involved, both in our predictions and in all our observations, that they can give rise to no recognizable predictive failures? Russell recalls what at one time seemed a whole group of such relations:

Kant asserted that [Euclidean] geometry is based on an a priori intuition of space and that experience could never contradict it because space constitutes a part of our manner of perceiving the world. 
A relation of Euclidean geometry. "The sum of the angles of a triangle is $180^{\circ}$." With straight lines defined by the paths of light rays, Gauss carefully carried through a large scale triangulation that showed this relation sound within the (narrow) limits of what could legitimately be regarded as experimental error. Kant notwithstanding, Gauss apparently considered that a failure of the relation would be recognizable. Even at the most fundamental level of our conception of space, a colligative relation would not then be so conventionalized as to be forever made "true." Indeed, once we agree on the denotations attaching to the conceptual entities of geometry, it becomes possible to prefer one geometry to another. In macrocosmic applications most physicists today prefer to Euclid's a Riemannian geometry in which the relation stated above is no more than an approximation.

\section{THE "PROOF" AND ENDURANCE OF COLLIGATIVE RELATIONS}

What is a legitimate generalization is not given us a priori, but always we seek maximum deployment of each relation. Not content simply to interpolate, we also extrapolate; e.g., we extend a graphed relation into regions where we do not have, and perhaps cannot get, any experimental points whatever. Such extrapolations we recognize as potentially hazardous; for whenever experience takes us into new and unfamiliar realms, as Bridgman observes,

. . . we must be prepared to find, and as a matter of fact we have often found, that we encounter phenomena of an entirely novel character for which previous experience has given us no preparation.

Yet, so profound is our confidence in the principle of continuity, we still remain bold enough to essay the most extreme extrapolations. At the very worst, we think, the relation will not blow up abruptly but fail only gradually-becoming a progressively poorer approximation. And, such is our faith in continuity, even today we are sometimes lamentably slow to recognize the failure, even as approximations, of apparently plausible extrapolations.

A theory may be harmful if it encourages us to forget that such extrapolations may prove fallible. But it is harmful, too, if it too strongly discourages generalization and extrapolation. Provided that we permit no relation to become permanently conventionalized, 
maximum generalization of a relation is clearly the optimal policy. Certainly it is the policy best designed to bring to light any inadequacies of the relation: "Give a man enough rope . . ." By generalization a relation is put to proof and, as we come sooner or later to recognize its limitations, its value to us is not impaired but very much enhanced.

We say: "Two things cannot occupy the same space at the same time." At first sight this statement seems perfectly general and perfectly reliable. But a great deal depends on how we understand the denotation of "things." We might eliminate some failures of the relation by excluding gases from "things"; with increase of pressure two gases can easily be crowded into the container formerly "occupied" by one of them. Some other failures we might eliminate by excluding liquids; still others, by excluding solids (in sodium sulfide the sodium and sulfur together occupy a volume less than that initially occupied by the sodium alone). Denying status as "things" to gases, liquids, and solids, out of fear of error we reduce the relation to naught. Actually, of course, we do nothing of the sort. Ordinarily, surely, volumes are additive-even in gaseous systems if they are nonreactive and held at constant temperature. Precisely as we recognize that the relation has a proper domain of application, it becomes a more reliable guide.

Certainly an ill-founded colligative relation may be discarded, as erroneous or fortuitous; and certainly a relation may be extensively reformulated to acknowledge (or surpass) previously unrecognized limits to its applicability. But de Broglie quite correctly stresses that once a relation has been properly authenticated-

... we have a definitely acquired result which no later speculation is able to undo. If it were not thus, no science would be possible. But it can very well be that, in the light of new experimental facts or of new theoretical conceptions, we are led to consider previously verified laws as being only approximate, that is to assume that, if the precision of the verification were indefinitely increased, the laws would not be more exactly verified. This has happened many times in the course of the history of science. Thus the laws of geometrical optics-for example, the rectilinear propagation of light-although having been verified with precision and at first regarded as rigorously true, were seen to be only approximations that day when the phenomena of diffraction and the wave character of light were discovered. 
Of course the laws of geometric optics retain all their wonted usefulness throughout the domain in which they had earlier demonstrated their efficiency. What we have discovered is simply when and where not to apply them and, in Duhem's view, this is the kind of discovery we must expect to make ultimately about any scientific law.

It is provisional because it represents the fact to which it applies with an approximation that physicists today judge to be sufficient but will some day cease to judge satisfactory. Such a law is always relative; not because it is true for one physicist and false for another, but because the approximation it involves suffices for the use the first physicist wishes to make of it and does not suffice for the use the second wishes to make of it.

After this we are forewarned not to use the law when we find ourselves in the position of the second physicist, but we continue to use it, as a limiting law, whenever we are in the position of the first.

Dalton arrived at his law of partial pressures by way of a theory of the nature of gases we wholly reject, but the law survives. Man-made theories rise and fall; man-made concepts and their denotations prove highly mutable. But colligative relations have generally an immortality denied their conceptual formulations. How can this be? Quite simply! The "invention" of human theoreticians who may well be wrong, once "discovered" the law exists independent of their wisdom or folly. By virtue of the denotations attaching to the conceptual terms in which it is formulated, the relation refers to observables that do not change when we change our ideas. Even if a law is given a new conceptual formulation, and new theoretic accommodation, what has correctly been said about the relation of observables by the older law must be said also by the newer. Thus, as Poincaré remarks, a law can survive as the imperishable memorial of a theory perhaps long since passed away.

At first glance it appears to us that theories last but a day and that ruins heap up on ruins. . . . But there is something in them which endures. If one of them has revealed to us a true relation, this relation has been acquired for all time. We shall find it again under a new cloak in the other theories which will reign successively in its place.

The relation of metals. "Metals, however dissimilar in other respects, are generally hard, dense, malleable, ductile, lustrous, in- 
soluble, destroyed by corrosive acids, etc." Apparently a definition of what we mean by "metals," this is a genuine colligative relation having the form of the sulfur relation: If a substance has several of the following properties ... then probably it is a metal and has the other properties. This relation antedates the rise of systematic chemistry, and survived for millennia without theoretic accommodation. Early in the 1Sth century the phlogiston theory proposed that metals be viewed as compound bodies with one component, phlogiston, common to all of them. The relation is then rationalized: the presence of the common component "explains" the occurrence of properties common to all. Toward the end of the 1Sth century, however, the advent of Lavoisier's new chemical theory destroyed the phlogiston theory. In Lavoisier's system all the metals are distinct elements, apparently having nothing in common. The relation is then no longer rationalized, but of course it survives the loss of its theoretic accommodation.

For more than a century the relation of metals survived, amply useful but an enigma. Only in the last half century has it acquired what we conceive to be an enduring rationalization. Metals, we now suppose, do contain something in common: the company of labile electrons that constitute the metallic bond. Once again we "understand" the relation-indeed if we read "electrons" for "phlogiston" we understand it, and many other such relations, in much the same way they were "understood" in the phlogiston theory. But through all such clouds of theoretic speculation the relation itself looms up and endures solid as a rock.

\section{Colligative Relations not Wholly Independent of Theories}

So far in this chapter, and particularly in the last section, we have considered colligative relations as completely independent of their theoretic affiliations. This simplification facilitates discussion, but must not be pressed too far. Typically, a colligative relation is doubly connected. By the denotations of its conceptual terms it is linked with experience; by its accommodation in a theory it is linked deductively to the postulates thereof. Either link may be formed first. The relation may first materialize as an empirical regularity, as did Boyle's law, and only subsequently acciuire theoretic accommoda- 
tion; or it may first appear as a theoretic deduction, as did in part Moseley's law, and only subsequently be shown to describe and predict experience. Whichever link is formed first, we rest dissatisfied until both have been forged. Without the link to experience there is no colligative relation. Without the link to theory it remains "unrationalized" and, so, hard to "grasp" and manage with the manifold others of its kind.

Even strikingly efficient colligative relations may then leave us dissatisfied as long as they remain uncorrelated by any theory. Evoking a determined effort to provide theoretic accommodation for all "purely empirical" relations, this healthy sense of dissatisfaction supplies an indispensable element in the internal dynamics that renders the scientific movement self-preservative. For science would surely and rapidly degenerate into natural history-into a chaotic and unmanageable welter of purely empirical relations-were it not for the characteristically strong and enduring endeavor that, in mature sciences, has kept to an absolute minimum the number of these uncorrelated relations.

A highly efficient relation, one that cannot possibly be dismissed as something that "just happens," will of course be used, preserved, and defended in any case. Thus, having lost its theoretic accommodation, the relation of metals survives until it finds a new one. And thus, though it had never any theoretic accommodation, the relation of inertial and gravitational masses survives during the more than two centuries that separated the work of Newton from that of Einstein. But in marginal cases our opinion of a given relation may be decisively influenced, in a way not so far indicated, by the extent to which it has (or has not) achieved theoretic accommodation. With faith in the principle of intelligibility, we believe that for all genuine relations such accommodation must be possible. Failing reasonably promptly to achieve accommodation of some relation of doubtful status, we will be very strongly tempted to dismiss it as an "accident" rather than permit the potentially disastrous accumulation of a multitude of unaccommodated relations.

As is clearest in solar eclipses, the ratio of the diameters of moon and sun as seen from the earth is almost exactly $1: 1$. This is certainly a relation of observables, and was at one time held significant. But this relation, unattractive in its meagre generality, is also one we find theoretically inexplicable; and we simply, and probably quite prop- 
erly, dismiss it as an "accident." As another example, consider how in the early 19th century Prout called attention to a relation (among chemical atomic weights) which, aside from a few notable exceptions, seemed too frequently approximated to be dismissed as an "accident." No generally convincing theoretic explanation of this relation having been given by the end of the century, most scientists were then inclined to dismiss it. Today, having acquired a theoretic interpretation, the relation is again deemed of some significance. Following is an even more striking illustration of the influence of theories on the appraisal of laws that seem to hang on the borderline between significance and happenstance.

The Bode-Titus law. In 1772 Titus announced the discovery of a relation among the orbital radii of the six planets then known. The nature of this relation is displayed in the accompanying table, in which the radial distances are expressed in tenths of an astronomical

\begin{tabular}{crrl} 
A series term +4 & Total & \multicolumn{3}{c}{ Observed orbital radius } \\
0 & 4 & 3.9 & Mercury \\
3 & 7 & 7.2 & Venus \\
6 & 10 & 10.0 & Earth \\
12 & 16 & 15.2 & Mars \\
24 & 28 & & \\
48 & 52 & 52 & Jupiter \\
96 & 100 & 95 & Saturn \\
192 & 196 & &
\end{tabular}

unit. The near equality of the numbers in the second and third columns is amply impressive. Is it significant? Bode, who thought it might be, set out to discover an as yet unobserved planet which, according to the relation, should be found between Mars and Jupiter. And to be sure Ceres and, subsequently, a multitude of other asteroids (presumed to be the fragments of an extinct planet) were found, by various astronomers, in precisely the region predicted by the relation. This, taken together with the somewhat earlier discovery of Uranus (which has an orbital radius of 192, within $2 \%$ of that predicted by the relation) seemed to place the Bode-Titus law beyond all doubt.

Subsequently the tide turned. Two outer planets, Neptune and Pluto, were found with orbital radii diverging $20 \%$ and $100 \%$, respec- 
tively, from the values predicted by the Bode-Titus law. In the face of these setbacks, and still lacking theoretic justification, the law was then dismissed as "accidental" by many astronomers, though by others it was still held a genuine relation distorted by conjectural "secondary effects." Recently the postulates of a revised nebular hypothesis for the origin of the solar system seem to yield the BodeTitus law as a deduction. If this promise is borne out, the law will no longer be dismissed as an "accident" and, conversely, its accommodation will become one of the strongest bits of evidence for the new hypothesis. Theory and law have here a powerful mutual interaction.

\section{CONNOTATIONS AS WELL AS DENOTATIONS}

A scientific theory may affect our appraisal of a given relation; conversely, a relation may help us to the first conception of a theory. Beyond predictively efficient denotations, one then draws from the relation theoretically suggestive connotations. To be sure, these connotations do not simply inhere in the colligative relation as such: only one, or a very few, of many who know the relation may fully grasp its implications. A Newton reads the connotation of Kepler's laws to be the action on planets of a sun-directed centripetal force; after more than two centuries an Einstein finds in the equivalence of gravitational and inertial masses a connotation previously grasped by no one. In the gifted mind even a crude or fragmentary relation may suffice to spark the flash of insight. But always the connotations of a relation will be most readily grasped when, beyond a discovery, it is also an optimal invention, i.e., cast in an abstract form well suited to theoretic consideration.

We saw that by the time we attain even as simple a relation as Boyle's law, we have already traversed a great, if not the greater, part of the road from "concrete fact" to "abstract theory." With the acquisition of Boyle's law a chaos is reduced to at least comparative order. Moreover, the law provides the reference standard that first permits us to recognize, and measure, the departure of real gases from ideal behavior. The theoretic connotations of these systematic deviations thus first become accessible only through the mediation of the abstract law that contributes to the organization of even the "imperfections" of which it renders no account. Consider now a relation of a still more abstract sort.

Moseiey's law. Let Z represent the "atomic number" of the element 
that. under specified conditions, emits light of "wavelength" $\lambda$ : for the $K_{\alpha} \mathrm{X}$-ray line we can then write $\lambda(Z-1)^{2}=1.21 \times 10^{-5}$. This seems so purely theoretical, so abstract, that our first obligation is to show that it can function as a colligative relation.

By 1869 the concept "relative atomic weight" had acquired a reasonably defined denotation. Given certain chemical data, one could compute "relative atomic weights"; given atomic weights, one could predict certain chemical data. Beginning in 1869 Mendeleev, Meyer, and others developed a classificatory chart in which, with a few specific inversions, the chemical elements are arranged in order of increasing atomic weight. The "atomic number" of an element is simply the ordinal number expressing its position in the sequence of the perfected classification. Giving exceedingly compact expression to a great many relations among measurable properties of the elements, Mendeleev's "periodic table" is in part "theoretical," e.g., in its implicit acceptance of the Daltonian atomic theory; and it has very pronounced connotations, e.g., the nonultimacy of the chemical "elements." But through it the concept of "atomic number" acquires a clear enough denotation. Given certain measurements made on an element and its compounds, I can assign it an ordinal number and a place in the chart; given the chart and the number of some element, I can state the probable outcome of certain experiments not before made on it.

Turning now to the "wavelength of light," in the X-ray region a denotation is fairly readily established with an instrument like the crystal reflection spectrograph. The "wavelength" of light passed into the instrument is calculated in a specified way from the location ( $\mathrm{s}$ ) at which blackening is produced on a photographic plate that forms part of the spectograph. An immense amount of theoretical work underlies the instrument and the calculation. But, once we accept this denotation, the concept has a perfectly clear linkage with experience. When I have seen the plate I can assign a definite wavelength $(\lambda)$ to the light which produced a black mark on it; and when I predict a certain value of $\lambda$ I say where on the plate I expect to see a black mark.

Now we can come to grips with Moseley's law. We "excite" an element under the specified conditions and, passing the light so generated into our spectrograph, we find a determinate relation between the ordinal number of that element in the periodic classifica- 
tion and the position of one identifiable mark on the photographic plate. Were we to decide that matter is non-atomic, and light nonundulatory, presumably our formulation of this relation would no longer be given in terms of "atomic number" and "wavelength." But even such a drastic upheaval could not alter the substance of this relation: between the position of the element in the periodic classification and the position of the mark on the photographic plate there is an indissoluble connection.

A physicist might rightly object that this is a preposterous way to put Moseley's law-not wrong but grotesquely insufficient. Dwelling on its denotations, we have wholly ignored the law's weighty theoretical connotations. Reading the abstract law in the light of the Rutherford-Bohr theory of the atom, we discover the basis for a profound rationalization of Mendeleev's "purely empirical" classification. $Z$ we identify as both the "number of protons" in the "atomic nucleus" and the "number of electrons" attendant thereto. One apparent failure of Moseley's law was then taken to connote the probable occurrence of an undiscovered element. That element (hafnium) was indeed discovered some years later, when excitation of a sample in which its presence was suspected did yield a spectrographic plate with a spot corresponding to the expected atomic number. This pretty piece of work offers a vivid illustration of the interplay of the connotation and denotation of an abstract law.

The Mendelian laws. The following will serve us as a particular example: "In monohybridization experiments conducted on a large scale, in which dissimilar parents are crossed and the first filial generation is intercrossed, three-fourths of the members of the second filial generation will exhibit the dominant forms of the differentiating characteristic." Let us assume what is not at once evident: that the denotations of the many conceptual terms here involved can be made adequately clear. The need for the qualification "conducted on a large scale" at once signifies that we have here to do with a new (statistical) kind of relation unlike any so far discussed.

No relation furnishes absolutely reliable prediction of a particular event in a particular system. However, when dealing with relations of the kind considered earlier, we find that predictive reliability can be increased without apparent limit-by improving the accuracy with which we determine the conditions defining the state of the system, and/or by broadening our definition of state to take account of a 
greater number of initial conditions. With a statistical relation, on the other hand, we may find that no such increase in the number of specifications of the state of the particular system suffices to increase the reliability of our prediction of the one particular event. Dealing with a statistical relation, we find only one way to improve our performance: we must limit our predictions to comparatively large numbers of events, and the improvement in predictive reliability is in proportion to the increase in the number of events. We are powerless to predict the result of a single throw of a well-balanced die, but we predict with confidence that in a long series of throws each of the faces will turn up very nearly one-sixth of the time.

Often a statistical relation leaves us profoundly unsatisfied: for example, each man seeks one specific item of prediction no statistical table of life expectancies can ever yield him. In exactly the same way, the Mendelian laws leave undetermined whether a given seed -with a given genetic heritage-will produce a white or a pink flower. But when we work with large numbers of seeds the laws permit us to make excellent predictions of the ratio of white and pink flowers. The table shows Mendel's own results for seven sets of monohybridizations conducted with sweet peas.

Differentiating

Characteristic

Second Filial

Generation

\begin{tabular}{lccc} 
& DOMINANTs & RECESSIVES & Ratio $\frac{\text { DOM. }}{\text { REC. }}$ \\
Form of seed & 5474 smooth & 1850 wrinkled & $2.96: 1$ \\
Color of seed coat & 6022 yellow & 2001 green & $3.01: 1$ \\
Length of stem & 787 tall & 277 dwarf & $2.84: 1$ \\
Color of flowers & 705 colored & 224 white & $3.15: 1$ \\
Position of flowers & 651 axial & 207 terminal & $3.14: 1$ \\
Form of pods & 882 inflated & 299 constricted & $2.95: 1$ \\
Color of unripe pods & 428 green & 152 yellow & $2.82: 1$ \\
\hline Total & 14949 & 5010 & $2.98: 1$
\end{tabular}

The relation so clearly evident in the table is one of very broad generality. It applies with equal facility to a great number of differentiating characteristics displayed by a great variety of plants and animals; and it furnishes a secure predictive guide of profound importance to plant hybridists and others who work on a large scale. 
But at this point our chief concern is with the Mendelian laws' connotations. These are, of course, enormous: from them derive the foundation blocks of classical genetics. And even the predictive failures of the Mendelian laws, in other than a statistical sense, are rich in connotations, e.g., as regards the linkage of genes in chromosomes.

\section{COLLIGATIVE RELATIONS IN OTHER GUISES}

Everything called a scientific law is not necessarily a colligative relation; everything serving as a colligative relation is not necessarily restricted to the function of a more or less efficient predictive device. The relation may have connotations that, as we have just seen, supply suggestive pointers for theory construction. Having achieved theoretical accommodation, the relation acquires a new footing in the heuristic apparatus, as something entailed by a theory there established. There, too, it may appear in generalized form not as deduction from postulates but as in itself a postulate; e.g., the conservation of energy as first conceived by Joule is a colligative relation before it becomes the first principle of thermodynamics. In the heuristic apparatus again the law may appear as a substantive principle, taken for granted and temporarily conventionalized while we use it in our study of other laws. Yet again, a law may be involved in establishing the denotations, and in particular the alternate "equivalent" denotations, of an indicative concept; e.g., Boyle's law becomes part of the definition whenever we rely on a McLeod gauge to measure a "pressure" too small to be measured with an ordinary manometer. Apart from any status as colligative relation, a scientific law may thus assume manifold guises and functions, some of which we shall examine later. 


\section{CHAPTER VI}

\section{Empirical Tools and Empiricism}

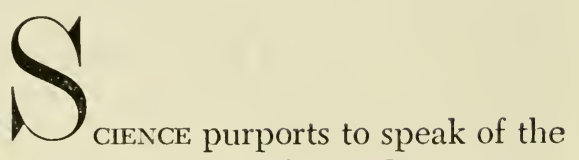

world of experience; the devices of empiricism safeguard its contact with that world. To be sure, not all scientific concepts are linked to observables with exactly equal clarity and directness. Thus, for example, many of the (explicative) concepts figuring in the postulates of highly abstract theories will be so linked only indirectly-by way of colligative relations derivative from those postulates. Nevertheless, among scientific concepts generally, we find a remarkable reconciliation, of great abstraction and great clarity of denotation, highly characteristic of science-because completely unparalleled in common sense, philosophy, mathematics, or any other human endeavor. At the most fundamental level this union develops from the power of the empirical devices deployed by scientists: their special materials and specimens, procedures and techniques, instruments and equipment.

\section{INSTRUMENTS}

In Chapter II we saw how instruments may facilitate the concurrence of multiple observers of the "same thing." You and I will agree on the magnitude of some particular "light intensity" as soon as we agree to adopt a denotation that points us toward a straightforward observable, such as the scale reading of a light meter. Even a notably abstract scientific concept may thus acquire a clarity of denotation 
immeasurably superior to that of the more "obvious" (i.e., familiar) concepts of common sense. The sophisticated concept of X-ray wavelength is invested with a comparatively unequivocal denotation, established around such a device as the crystal diffraction spectrometer, and "nuclear spin" one established around the NMR spectrometer. Of course, the situation is not completely uncomplicated: beyond the instrument itself we have also to specify the protocol for its "proper" operation.

The operating protocol. Need any appreciable procedural specification be given in the simplest of cases, e.g., an accurate measurement of "length" with a meter stick? Yes-at least two considerations demand attention.

First: If the stick and the object to be measured are not in the same plane, then at oblique angles of view we may read from the meter stick substantially erroneous lengths for the object. Grasping this possibility of (parallactic) error, we easily avoid it-perhaps by bringing into play another instrument. Thus a cathetometer can assure us of a perpendicular line of sight and, by a suitable arrangement of lenses, allows us to cast the object and the comparison scale in the same focal plane. But even then we do not attain a purely instrumental denotation: "proper" operation of the cathetometer itself demands a protocol specifying a considerable variety of procedural details. And so on.

Second: The meter scale has a length of one meter only at the specified temperature at which it was graduated. Often, however, we seek an accurate result from measurements we must make at some other temperature. Then, using what we regard as a wellestablished relation between the temperature and the length of the scale, we calculate and report, as the accurate length of the object, a "corrected" value more or less different from what we actually read from the scale. That such corrections must be made, and the colligative relations with which they are to be made, we learn from the protocol thus required to fill out the denotation of "length" even after we have agreed to center that denotation on an instrument, the meter stick.

The need for an auxiliary protocol is perfectly general: "length" is an atypical case only in the comparative simplicity of this prescription. Consider the multiplication of complexities in an instance only slightly less simple. Suppose that, for some liquid, we wish to meas- 
ure accurately the "boiling temperature"-and adopt a thermometer as the instrumental core of denotation. Do we then simply read off the temperature shown by a thermometer immersed in the boiling liquid? Not at all! We find that, due to "superheating," a boiling liquid stands at a temperature irreproducibly higher than that of its vapor. We then specify use of a "boiling point apparatus," in which we arrange to measure the vapor temperature, following a procedure designed to minimize superheating of that vapor. We have now an instrument, an apparatus, and a procedure-but at most only part of the procedure: the actual thermometer readings will require "correction." Using a well-authenticated colligative relation, we make a "stem correction" to allow for the fact that, although the mercury in the thermometer bulb is at the temperature of the saturated vapor, the mercury in the stem is ordinarily at some other, lower, temperature. Another colligative relation warns us that boiling temperature is a function of pressure: we must then correct the thermometer reading to allow for the "barometric pressure." But now, to establish this last, we will require still other corrections. One such correction term is a function of the temperature of the barometer, which affects both the density of the mercury and the length of the measuring scale; another involves the latitude and elevation of the laboratory, which affects the gravitational acceleration, and hence the formula for the conversion of barometric heights to barometric pressures. And so on. Without going any further, we see clearly that establishment of the denotation of "boiling temperature" requires a procedural protocol of substantial complexity.

How grave are the risks that the scientist will be led seriously astray by the element of human subjectivity irreducibly involved whenever, by making "corrections," he ventures to "tamper with the facts"? The relations used in making corrections have been independently checked many times over. Moreover, in some crucial cases we have made more elaborate experiments in which the need for many if not most corrections is eliminated. In these cases we have generally arrived at the very same results obtained much more conveniently in simpler experiments involving multiple corrections. A second point: corrections may enhance predictive reliability. For example, Dulong and Petit's law we now know to be only a crude approximation. We find it a better approximation when we use as specific heats not the values actually measured, ordinarily at con- 
stant pressure, but corrected values hypothetically representing the results of constant-volume experiments. Conversely, when we make due allowance for this correction, Dulong and Petit's law becomes able to furnish more reliable predictions of what we will observe in new cases, cases not before examined. Third, and perhaps most important: however less objective they may or may not be, the corrected values are certainly very much more impersonal. Different scientists measuring the "same thing" under different conditions observe different raw values, which each subjects to the corrections appropriate to his own experimental conditions. Only at the level of the corrected values is general agreement reached, and the attainment of such agreement cannot but strengthen our confidence in the correction terms. For there is nothing "artificial" or contrived about this impressive concordance: the correction formulae involved in the operating protocol are established before we know the actual values of the measurements reduced with their aid to agreement.

No matter how the concurrence of observers is facilitated by use of an instrument, the instrument always constitutes at most the core of denotation. We never achieve completely explicit categorical denotations. The denotation always must be filled out with an operating protocol involving words and symbols for proper understanding of which we must, ultimately, rely at least in part on the "good judgment" of the investigator. He is trained to such judgment by education, by the editorial policies of his journals, and by the example of distinguished colleagues. So pervasive are these influences of the international community of organized science that ordinarily we need not even state the auxiliary protocol that completes an instrumental denotation. Ordinarily we safely assume that protocol "understood" by all competent and responsible scientists-for of course it is simply shaped to take account of colligative relations that find a place in the theories known and accepted by all. In using this particular instrument, I design my experiment and correct its results in a way determined by my awareness of the effect of other variables on the parameter I seek to measure. Thus, for example, knowing the relation between barometric pressure and boiling temperature, all scientists will duly allow for the first when determining the second. The denotative uncertainty introduced by the necessary, if wholly tacit, involvement of an operating protocol is, then, ordinarily negligible.

Pointer readings. Still yearning to establish completely categorical 
denotations, we may think to do so with the aid of more elaborate "self-sufficient" instruments. Imagine a device in which insertion of a sample at one end is followed by automatic production at the other of a pointer reading giving "the result." The operating procedure is then reduced to plugging in the instrument and inserting the sample; the need for corrections is eliminated because the instrument itself provides stabilization of the conditions of measurement, or contains compensatory mechanisms for making the corrections automatically; and the result given as a pointer reading seems to be the non plus ultra of denotative clarity. Have we then at last arrived at a fully categorical denotation?

Alas, we have not. We now require a protocol for detecting and repairing malfunction of the "self-sufficient" instrument. Far from eliminating all auxiliary protocols, we have simply substituted a new one for the old-and the new one is ordinarily much the more difficult. This protocol cannot safely be left to the "good judgment" of the investigator. Through an elaborate instruction manual, the manufacturer of a complex instrument supplies an operating protocol as explicit as he can make it and, beyond this, he must make available also the services of the specialists required to rectify malfunctions of the instrument. In the long term, then, such an instrument may make demands on human judgment not less but greater than those imposed in use of simpler devices.

Through instruments we may first gain access to realms of experience otherwise outside our ken. The microscope "amplifies," the NMR spectrometer "converts," hypothetical signals to which our senses are dead into signals to which they are very much alive. In addition, the automatic pointer-reading instrument may offer great advantages in speed, in sensitivity, and in simplicity of routine operation-which can often be entrusted to unskilled technicians. These are immense gains, justifying every effort to achieve them. But, as Bernard well recognized long ago, the introduction of elaborate instruments where they are not needed is positively disadvantageous.

. . . we need to learn that the more complicated the instrument, the more sources of error does it create. Experimenters do not grow great by the number and complexity of instruments. Quite the contrary. The great experimenters, Berzelius and Spallanzani [and Bernard, Pasteur, and Rutherford], made great discoveries by means of simple instruments. 
Even today in certain applications the human eye offers us discriminatory capacities unmatched by any instrument or combination of instruments. Why should we hesitate to make the fullest possible use of the sensory faculties with which we are endowed? A vain lusting after reduction to instrumental measurements in general, and to pointer readings in particular, seems to rest on complete misconception of what we gain with their aid. Not categorical denotation, not the complete elimination of human judgment, not "objectivity." The fundamental achievement is this: denotative clarity is enormously enhanced by adoption of an "external" rather than an "internal" standard of reference. Though instruments and pointer readings are generally sufficient to ensure this great advance, they are not always necessary to ensure it.

\section{THE EXTERNAL STANDARD OF REFERENCE}

How shall I establish the denotation of "weight"? That denotation remains highly uncertain for just so long as it is "fixed" only by an internal standard, i.e., the sense of muscular strain felt in lifting. In that case I have always to remember what it felt like to lift other "weights," and my memory is not wholly reliable. Nor are my sensations unaffected by my recent personal history: things may well seem heavier when I am tired. My own estimates of the weight of a given. object vary from time to time, and disagree with the estimates made by other persons who rely on their internal standards.

The self-consistency of my own reports of weight, and the concurrence of different reporters, are both enormously improved when we all agree to adopt one particular set of standard weights. "Weighing" is then reduced to making a comparison between one or more of these and the unknown weight. I may, for example, seek to make some combination of standard weights that "feels the same" when it is picked up immediately before or after the unknown weight-the magnitude of which can then be expressed in terms of the stand$\operatorname{ard}(\mathrm{s})$. As already indicated (p. 51), I can even go somewhat further. Making comparison not through trials by lifting but with an equalarm balance, I exclude secondary complications that might arise when unknown and standard differ sharply in shape, texture, etc. In this way the role of human judgment, though never completely eliminated, is still further diminished and simplified-while at the same time we acquire a delicacy of discrimination wholly unknown to the 
unaided senses. To go still further-to an elaborate, automatic, pointer-reading device-may win us gains in sensitivity and convenience, but can afford us no further fundamental gain in clarity of denotation.

Consider the analogous case of "temperature." Our thermal sensibility, quite limited at best, is also notoriously unreliable; it is, for example, heavily dependent on the recent history of the sensing surface. The denotation of temperature is immensely clarified and refined with the aid of a thermometer. At first sight this instrument (unlike the equal-arm balance) seems to involve no comparison with an external standard. The balance is a null-reading instrument involving an explicit comparison, but scale-reading instruments like the thermometer normally involve implicit comparisons. The scale is constructed by comparison (direct, indirect, or by way of colligative relations) with certain external standards. Thus whenever I read a temperature from a thermometer scale, I make an implicit comparison with, say, the temperatures of melting ice and boiling water. This state of affairs remains unaltered when the instrument is more deeply involved, in amplifying and/or converting an otherwise undetectable signal. An ohmeter gives a direct pointer reading of "resistance," but ultimately it yields a comparison of this resistance with that of a particular spool of manganin wire.

Denotations depending on "absolute" judgments-that is, judgments made relative to more or less uncertain internal standards-suffice for many purposes, e.g., counting. For some purposes, e.g., psychiatric diagnosis, they may well be indispensable. But, in general, denotations are most firmly established when we need make only relative judgments, i.e., comparisons with external standards. Thus, for example, the human eye is thoroughly ineffectual in judging the absolute intensity of illumination, but highly adept at distinguishing small differences in intensities that occur, or can be brought together, in the same visual field. Given one "standard candle," comparisons founded on use of even so primitive a device as the Bunsen greasespot photometer invest "light intensity" with a well-defined denotation. Hebb's comment amply suggests that we here encounter no trivial shortcoming of optic physiology but, rather, a general psychological phenomenon:

Man or animal tends to perceive relative rather than absolute intensity, extent, or frequency. One can readily train an animal to 
choose the larger of two surfaces; it is extraordinarily hard to get him to choose a particular size, except when differences are very great.

Just so, many of us easily detect a flat note in a continuing melody, but very few of us possess absolute pitch.

At one extreme of the spectrum of denotative clarity, the concepts of physics most often have precise, instrumentally established denotations. At the other extreme, few instruments are deployed in psychiatry, but the notorious nebulosity of its fundamental concepts stems ultimately from an almost complete (and unavoidable?) failure to create an acceptable body of external standards of reference. In the broad spectrum of denotative clarity the position of a given concept is then primarily correlated with the availability of such standards, and only secondarily with the availability of the instruments that make comparisons. There is no magic in the instrument as such. Where no external standard exists, no instrument can help us; and, however convenient in practice, the instrument is wholly superfluous in principle where we do not need it to make adequate comparisons.

"Things" as standards. A standard weight is a reference "thing" used in quantitative comparisons, but in many other cases only qualitative comparison is required, or even meaningful. Wishing to know whether a given specimen "belongs" to some previously described species or subspecies, the taxonomist supplements his mental image of the possibilities by making point-by-point comparisons between his specimen and those available in museum collections. Due to the intrinsic variability of living organisms, high-precision quantitative comparisons are here unlikely to be helpful. The taxonomist must then exercise his judgment within a penumbra of uncertainty the extent of which is, however, very much reduced by the availability of reference "things."

Sometimes almost purely qualitative comparison is completely definitive. Consider an organic chemist who wishes to know whether a synthetic compound $(X)$ in his test tube is or is not a certain previously isolated natural product $(P)$. With the aid of elaborate instruments he can compare various properties of $X$ and $P$, but actually he regards as most definitive a procedure involving only the simplest of instruments, and not even requiring quantitative measurements. This is the technique of the "mixed melting point." Having found 
that $X$ and $P$ melt at the same temperature, the chemist prepares a mixture of $X$ and $P$ and determines its melting point. If this is unchanged he concludes that $X$ and $P$ are identical. To determine the melting points a thermometer is ordinarily used, but this is superfluous in principle. The chemist need only prepare three similar tubes containing respectively $X, P$, and a mixture of $X$ and $P$. If he finds that they all melt at very nearly the same time in a slowly-heated and well-stirred "melting point bath," he then feels well justified in identifying $X$ as a specimen of $P$. With or without use of the thermometer, the denotation of the concept of $P$ is firmly established by the one reference specimen of $P$ and the technique of mixed melting point.

Techniques as standards. Even in the absence of a reference "thing," a technique or group of techniques can constitute an adequate external standard-as when Lavoisier established the denotation of "element" as follows:

The last term at which analysis arrives, all the substances which we have as yet been unable to decompose by any means, are elements as far as we are concerned.

What is to pass as an element is here fixed in terms of the techniques for analysis available at the time. What is taken to be an element may thus become a function of time, but at any one time we well know what does pass as an element. Invented in antiquity, the concept "element" developed its full usefulness only after Lavoisier thus provided a firm link between the abstract concept and the world of experience. Often deceptively simple in retrospect, the forging of such a link can, as here, represent a major step forward. A similar if less spectacular advance was wrought by Proust, who first suggested that techniques of analysis can serve also to establish the denotation of the concept "compound": a material is a compound if, wherever and however it is obtained, analysis finds it to contain its component elements in an invariant proportion.

Like all other denotations, those established in terms of techniques fall short of absolute clarity. Ordinarily the modern chemist still chooses to treat as an "element" a material that analysis, broadly conceived, finds resolvable into distinct (isotopic) components; and as a "compound," a material slightly variable in composition for this and other reasons. These usages present no real problems, but borderline cases severely strain the denotative precision of some 
other concepts. Asked to decide whether a given specimen is a "living organism," the microbiologist applies various culturing techniques to obtain results that ordinarily support an unequivocal answer. But when the crystalline viruses were first encountered, a latent indefiniteness of the denotation of "living organism" became quite painfully manifest.

Colligative relations as standards. Although we have discussed separately different species of external standards, most typically what we work with is a mixed standard. Beyond "things" and techniques a mixed standard involves colligative relations, which may indeed constitute the ultimate foundation of the entire standard. If an instrument of comparison is used, such relations determine its design and operating protocol. "Things" accepted as standards are selected with a view to the relations in which they figure, and techniques accepted as standards are shaped by such relations. Ultimately the denotation of "living organism" is established on the group of colligative relations expressing certain uniformities in the behavior of "living organisms" in various circumstances. (Perhaps this is the useful sense of the otherwise completely circular "law of definite proportions": A compound contains its component elements in an invariant proportion.)

Ordinarily I can safely rely on my judgment, i.e., on an internal standard, in deciding that a particular object is a "lever" or a particular spot of light is a "planet." But in doubtful cases a suitable relation can by itself constitute an exemplary external standard. One or a very few trials suffice to show whether the object does act in accordance with the law of the lever; a relatively small number of observations suffice to show whether the spot of light moves in accordance with Kepler's first and second laws. Assuming affirmative indications, we identify the object as a lever and the light as a planet. Serving thus as reference standards, the relations are not in any sense "lost" to us: with them we can now go on to hazard predictions beyond what we have observed.

\section{PRIMARY AND SECONDARY DENOTATIONS}

There is only one standard kilogram, but many kilogram (and subkilogram) "weights" are actively in use. All these are, ultimately, referred to the mass of a particular platinum cylinder kept in the vaults of the International Bureau of Weights and Measures, near 
Paris. With this standard are compared the kilogram masses maintained by the various national bureaus of weights and measures; with these masses, in turn, are compared the standards used by commercial manufacturers of "weights"; and so on in a kind of apostolic succession. Even as we thus multiply our standards, we multiply our instruments, taking care to assure their mutual consistency by similar cross-comparisons. Generally we venture much further still, bringing into play entirely new kinds of instruments that yield us powerful new secondary denotations.

Calibration. Taking as example the concept "electric charge," I simplify by considering only comparatively large charges passed in D.C. systems. The fundamental unit, the coulomb, is defined (temporarily) as the charge which deposits 0.00112 gram of metallic silver when passed under specified conditions through a specified device: the silver coulometer. Smaller or larger charges are measured as proportionately smaller or larger deposits of silver. The specifications of the apparatus and procedure are so clear and complete that the concept "electric charge" here acquires all the unequivocality one hopes to find conveyed by a well-contrived primary denotation. But-as everybody who has used a silver coulometer will testify-operation of the device demands the expenditure of a good deal of time, effort, and irritation. The silver coulometer is not a highly practical device. Often we prefer to use in its place an instrument as different as, say, some type of integrating galvanometer in which charge is determined by the measurable extent of the mechanical displacements its passage produces. A sophisticated electromagnetic theory may be required first to suggest the possibility of such an instrument. But entanglement of this theory with the denotation of "charge" is readily avoided -by the simple expedient of calibration.

We connect an integrator-galvanometer "in series" with a silver coulometer, so that the "same charge" passes through both. The observable correlation of the results yielded by the coulometer and by the galvanometer supplies the sought-for calibration of that particular galvanometer. From the galvanometric measurement we can then calculate the result that would be given by the less convenient coulometric measurements we need now no longer undertake. Continuing our studies, we arrive ultimately at a general result of still greater value. We find that the values yielded by the coulometer correlate with the readings afforded by all galvanometers of the same 
type, through relations involving only certain independently determinable characteristics of the galvanometer, e.g., the dimensions, arrangement, and number of windings in its coil, the strength of its magnet, the disposition of its mechanical system, and so forth. With these colligative relations in hand we can now establish an independent secondary denotation for "electric charge." That is, making no reference to silver coulometers, we can build integrating galvanometers and graduate their scales to read directly and accurately in coulombs.

Extrapolation. The way now lies open to a gain far weightier than mere operational convenience. Each "equivalent" denotation reflects the existence of one or more colligative relations joining different sets of data. Throughout the broad range in which galvanometric and coulometric results can be compared, we find them well correlated by relations we then dare extrapolate to situations in which only galvanometric measurements can be made. Provisionally conventionalizing the relations-incorporating them in my definition of charge-I graduate my galvanometer dial to read "electric charge" on a scale I assume everywhere consistent with that originally established with the coulometer. I can now extend the application of that concept to systems in which the charges are too large, too small, or too abruptly delivered to be measured with the silver coulometer.

All colligative relations somewhere fail of reliability. With the accommodation of the relations in theories, we may acquire some helpful indications. We may thus be warned not to extend "equivalent" denotations in circumstances demanding extrapolations that would run counter to approximations used in the theoretical derivation of the relations of equivalence (see p. 59). Our theories may encourage us to risk certain other extrapolations, but some element of risk persistently remains. We accept that risk to make this notable gain: important concepts may thus be made available for use far beyond the domains of their original applications.

Consider the case of "temperature." Today our scale of temperatures is pinned to certain fixed points established with the aid of gas thermometers. Applying certain corrections, suggested by theoretical (thermodynamic) considerations, we use such thermometers throughout the range from ca. $-265^{\circ} \mathrm{C}$ to ca. $+1200^{\circ} \mathrm{C}$. For the sake of convenience, we accept also many alternate denotations established with such devices as liquid-in-glass thermometers, resistance ther- 
mometers, thermocouples, and the like. Calibrating these at the fixed points, we contrive to assure the equivalence of the alternate denotations of "temperature."

How shall we extend the range of application of the concept "temperature"? The extension above $1200^{\circ} \mathrm{C}$ presents no serious problems. Here we use a radiation pyrometer that can be calibrated, at fixed points established with the gas thermometer, over a range of several hundred degrees. We then extrapolate the relation between pyrometric and thermometric readings into the range where only pyrometric measurements are possible. This extrapolation proves extremely useful and generally satisfactory; i.e., the data so obtained "make sense." But now consider the extension below ca. $-265^{\circ} \mathrm{C}$. As before, we extrapolate a relation, between the readings of some device and the readings of the gas thermometer, into the range in which only the new device can be used. Here a drastically different situation materializes: most of the attempted extrapolations prove unsatisfactory, and we easily recognize their inadequacy. That is, the "temperatures" so secured "don't make sense." We encounter discontinuities, inversions, and anomalies difficult to explain save on the assumption that most of the extrapolations were ill advised. Fortunately some extrapolations survive this test, and with them we make a deep penetration of the region close to absolute zero.

Many such cases give abundant evidence that in the long term any failures of denotative equivalence, due to unsound extrapolations of the relations of equivalence, will become recognizable and rectifiable. Thus even a primary denotation may be called into question. Today we adopt the gas thermometer to establish a scale of temperature we consider far superior to those of the liquid-in-glass thermometers originally primary only as a matter of historical accident. A comparatively recent revision of the primary denotation of "electric charge" now pins it to an electromechanical device (distantly related to an integrator-galvanometer) instead of the silver coulometer. What is primary and what is secondary may thus change with time, and often the distinction loses its clarity. To be sure if-inside the range in which both primary and secondary denotations are deployable-we encounter some inconsistency between the measurements they yield, we will adjust the relation between them to bring the second into conformity with the first. But, meeting contradictions and anomalies outside the range in which direct comparison of suppos- 
edly equivalent denotations is possible, we are thrown back on our own appraisals of the reliability of different extrapolations. We seek then to readjust the denotations we have assigned to our concepts until once again the data collected do "make sense." Proceeding so we do not wholly give way to human caprice: to "make sense" of experience $i s$, after all, the business of science. Nor do we in any way turn our backs on "hard reality": readjusting our denotations, we simply find that we can make sense of the data collected in one way and not in another. And always, whatever the risks that in future we may have further to reconstruct the denotations of our indicative concepts, we hold firm that indispensable bridge that science ever maintains between the conceptual realm and the perceptual realmhowever vertiginous may become the first, and however extended the second.

\section{Observation and Experiment}

Epistemologically the distinction between observation and experiment is a thin one. However complicated the equipment we bring to bear in the laboratory, the ultimate operation in any experiment is always the making of one or more observations. The ancient astronomer, making naked-eye observations of the sky, and the modern physicist, taking "pointer readings" from his "counter," are engaged in epistemologically equivalent undertakings. However, the important point is not this trivial equivalence but the enormous advancement of our conceptual powers produced when we pass from observation to experiment. In observation we can note only what occurs in the "natural course of events." Passing to experiment, we can for the first time observe what happens in a multitude of other circumstances we ourselves contrive. Though these conditions be "unnatural," the data we obtain may notably advance our understanding of what happens "naturally." Thus we learn much about the normal function of the endocrine glands by studying the abnormal conditions of organisms from which the glands have been removed by surgery.

Experiment cannot produce, but sometimes practically evokes, the concepts we alone can create. Thus movement observed in nature, rapidly suppressed by frictional effects, suggested the thought that rest is the "natural state" of bodies. Had it been possible to observe movement in the laboratory under conditions that minimize fric- 
tional effects, the creation of the modern (Newtonian) concept of inertia might not have been so long deferred. Today such laboratory experiments help even the novice to a firm grip on the concept which, without the aid of experiment, was not fully grasped even by Galileo. Of course the creation of this concept long antedated the laboratory production of any approximation to inertial motion. But sometimes simple laboratory experiences have clearly furnished powerful and suggestive stimuli to concept-creation. Boyle's conception of a "spring of the air"-a springiness later quantitatively expressed in Boyle's law-is prompted by the physical sensations felt when operating a laboratory vacuum pump. Faraday's concept of "lines of force" seems fairly to leap from the pattern observed in the distribution of iron filings around a magnet and, more generally, Born remarks that:

The revolutionary conception which distinguished electrodynamics from classical mechanics is that of the field. One can see in Faraday's work how it sprang from his observations of dielectric, paramagnetic and diamagnetic properties; . . .

Such clean-cut cases are presumably quite rare, but in another sense experiment practically always renders our thinking easier and more secure.

We gain new conceptual power as we pass on from observation to experiment but, Sambursky notes, we will essay that passage only when we have come to accept the principle of dissolubility much more fully than did the Greeks.

The essential thing in an experiment is the isolation of a certain phenomenon in its pure form, for the purpose of studying it systematically. Herein lies its artificiality. Natural phenomena occur as part of a web of interwoven and interconnected processes; their continuity in time and space makes them appear to us a single complete unit. . . . [Early experiments in mechanics by Galileo and Newton] were all based on the notion that friction or the resistance of environment are to be considered as incidental interferences with the study of the phenomenon that illustrates a natural law or principle in its pure form. This conception is as different as could be from Aristotle's. For him the environment was actually an integral part of the phenomenon itself, and he regarded the very idea of isolation as untenable.

Pushing to the extreme that dissection of Nature envisioned by Bacon, in the laboratory we leave behind us the complexity of the 
interlocked phenomena of nature, and seek escape to experimental systems that better approximate the simple "ideals" figured in our conceptual abstractions. From raw phenomena-complicated by multiple accessory effects and subject to the action of a host of variables difficult to identify, much less to evaluate-we seek escape to experimental systems in which, by isolation and control, we suppress the accessory effects, diminish the number of active variables, and make the remaining variables amenable to our adjustment. How much more easily we grasp the function in nature of a biochemical intermediate when, in the laboratory, we can study it in a wholly unnatural state of high purity! How much easier it becomes to establish the conjectural effect of a hypothetical variable when we can make it the only variable! And, more generally still, given reproducible laboratory data, we must always find it simpler "to see the thing" because, to some degree, at last we can see the simple thing.

By such experimentation on "parts" we acquire the understanding with which, often, we can mount a successful attack on the still formidable problem of giving a complete conceptual reconstruction of the integral phenomenon-which is so completely refractory when subject only to observation as such. Consider Pasteur. He finds the souring of milk far too complex and irreproducible to study directly. He elects to study this fermentation in a medium constituted of chalk, water, sugar, and yeast extract, to which he adds no more than a pinpoint's worth of material derived from milk. He proposes to grasp the essence of what happens in milk by studies made on the more controllable medium that, in effect, contains no milk. But then, having completed his studies on "sugared yeast water," Pasteur finds it possible to return to the souring of milk with an understanding made manifest by his ability to predict its vagaries and, most important of all, to conceive the possibility of something wholly new: the process we know as pasteurization. Precisely in the overwhelming success of such a return-from an ever-so-remote realm of superficially absurd conceptual and experimental abstractions-the scientist's capacity ever to maintain contact with the natural world is most convincingly demonstrable.

Goethe criticizes Newton for having gone into a darkened room to study light. This criticism was made when the empirical tools of science were still relatively simple. Today, when scientists devote so much of their attention to subtle effects unobservable without the aid 
of complex machinery-today Goethe's criticism can be put with much greater force. Do scientists grope in the dark for a conception of light? Do they turn their backs on the "real world" to study a world of purely synthetic phenomena artificially produced in a laboratory purposively cut off from the light of day? Can it be that what they study experimentally are not "natural" phenomena, but some artificially contrived experience they do not even accept as such, but "correct" as they deem necessary?

Undeniably scientists sometimes confuse an artifact of their laboratory machinery with a manifestation of nature. Eddington suggests a vivid illustration of this possibility: a hypothetical naturalist draws a net with one-inch mesh through the oceans of the world, and concludes from his catch that nowhere in the oceans are there creatures less than one inch in length. That scientists have suffered analogous, if more sophisticated, delusions is readily demonstrable. But the crucially important thing is that such delusions ultimately become recognizable as such. We learn, for example, to make "corrections": a half-fish in the net need not connote a half-fish in the ocean, but only a whole fish the other half of which has disappeared into some voracious companion with which, in the net, the victim has been brought in "unnatural" propinquity. Even more to the point, only a hypothetically stupid naturalist could forever content himself with superficial observation. The smallest modicum of experimentation reveals that inside the smaller fish in the net there are still smaller fish. Unpacking the Chinese box of successively smaller organisms, we would then ultimately obtain, from the catch of the one-inch net, a quite substantial knowledge of the microflora and fauna of the ocean.

Our experimental tools do not of themselves manufacture experience. The otherwise unattainable experience they make accessible to us must derive ultimately from nature. Consider, as an example, how we seek clues to life in the ashes of death. To discover what makes a plant thrive, we pluck it from the field, carry it to the laboratory, kill it, incinerate it, and analyze the ashes to find an answer. How absurd. But such studies, made by de Saussure and others, did indicate the chemical elements that figure in the composition of plants. With this knowledge we return from the laboratory to the fields, bringing with us artificial fertilizers that work a notable increase in agricultural productivity. The "unnatural" endeavors of the laboratory have then taught us something about nature. Surely we 
have not yet found the "secret of life." But in the ashes of dead plants, in the dark of the laboratory, we have found the ideas that permit us to attain for plants a more vigorous growth, and for ourselves a life of greater abundance.

\section{EMPIRICISM AND THE INTERNAL DYNAMICS OF SCIENCE}

By determining the accessible elements of experience, and the quality of attainable data, empirical tools contribute to the shaping of scientific history. A new tool can reveal a whole domain of experience the very existence of which was previously unsuspected. Here the telescope and microscope are paradigmatic. Note, however, the far more recent unforeseen discovery of that world of bizarre phenomena taking place at ultra-low temperatures. That science has been the "endless frontier" is due in no small measure to the function of its empirical tools in reconstructing its horizons.

No scientific Alexander need sigh for new worlds to conquer. Nor need he even await the discovery of new worlds: always there are known lands to which we first gain entry only with the development of new empirical tools. For long the geologist could only hypothesize certain phenomena inside the earth, but with the development of laboratory equipment generating extremely high pressures and temperatures many of these processes became observable in detail. The physiologist was aware that electrical phenomena accompanied the function of the brain long before the development of adequate electronic instrumentation finally made these phenomena accessible to study.

Science may be given a dramatic turn even by the quite modest empirical innovation that offers no more than easy entry in practice to a realm of data already accessible in principle. Consider as example the recent development of high-speed computing machinery. What is the magic of the computer? This elaborate machinery can perform no mathematical operation previously unperformable by a human mathematician provided with pad, pencil, and plenty of time. Plenty of time, and that is the point: the computing machine can do in seconds what a human computer would need years to accomplish. And from this advance in speed many major lines of theoretical investigation have derived immense impetus. As an instance totally different in character, yet intimately related in effect, consider what progress was opened to genetics when it was fortunate enough to choose as its 
prime experimental subject the fast-breeding Drosophila melanogaster, possessed also of giant chromosomes and many differentiating traits. Consider finally how whole areas of modern biochemical research have been galvanized by the development of the extraordinarily simple but powerful techniques of filter-paper chromatography.

The devices of empiricism represent a major factor in the internal dynamics of science. From them the scientific movement gains not only general trends and directions but also specific foci. A new empirical device draws attention to itself. At the frontier of knowledge to which it provides access, great prizes may be won by the hardihood of pioneers. A potentially powerful tool then produces the state of mind of the gold-rush: from all contiguous areas "everbody" hastens into the country newly opened. Today "everybody" interested in structural chemistry seeks to exploit the novel powers of NMR and EPR spectrometers. Some two centuries ago "everybody" was attracted to the study of certain "odd" materials (e.g., phosphorus, mercuric oxide, hydrogen), and in such studies "everbody" sought to deploy the simple apparatus and techniques of pneumatic chemistry. Out of this high local concentration of activity developed the "Chemical Revolution."

Bartlett remarks that:

It is vastly important to realize how much experimental thinking is controlled by experimental method and by experimental instrumentation and how hard it is, once methods and instruments have become accepted and established, to break away from their use.

No small part of the history of science is written in the history of the devices of empiricism. But to suppose empiricism self-sufficient is surely absurd. Empirical devices help to establish the application of our ideas, the denotations of our concepts; but judgment and "good sense" are still requisite. By widening the realm of our experience, by providing us with experience of peculiarly simple (experimental) systems, empirical devices can function even as aids to thought. But never can they function as substitutes for thought. Without Tycho's fundamental improvements in the instruments and techniques of observational astronomy, and without the wonderful body of data collected by Tycho, Kepler could not have discovered his celebrated 
laws. But Tycho's empirical data do not themselves proclaim Kepler's laws: to discover them the conceptual insight of a Kepler is requisite.

\section{The Scientific Method}

Empiricism, fact, and logic loom large enough in science to give some color to the popular conception of a "scientific method" (sometimes even The Scientific Method) infallible because it relies on nothing but systematic empiricism, hard fact, and cold logic. But this alluring conception of routinized Method is indefensible in the face of multiple objections, not the least being Polanyi's comment on just those great turning points that have made the history of science what it is.

Major discoveries change our interpretative framework. Hence it is logically impossible to arrive at these by the continued application of our previous interpretative framework. . . . by the diligent performance of any previously known and specifiable procedure.

Consider too that, given Method, the advance of science should be as smooth and unfaltering as, indeed, it may appear in the long-term perspective of textbook presentations which are analytical rather than historical. Conant, however, remarks that:

The stumbling way in which even the ablest of the scientists in every generation have had to fight through thickets of erroneous observations, misleading generalizations, inadequate formulations, and unconscious prejudice is rarely appreciated by those who obtain their scientific knowledge from textbooks. It is largely neglected by those expounders of the alleged scientific method who are fascinated by the logical rather than the psychological aspects of experimental investigations.

Even cursory scrutiny of the actual historical record-the modern no less than the ancient-yields ample evidence that in close-up perspective the advance of science is sporadic, laborious, tortuous.

Given Method, there should be a major element of sameness in the practice of those to whom science is indebted for its major advances. Examination of the historical record reveals not sameness but overwhelming diversity. At the very least we might expect to find unanimous acceptance of the basic tenet of empiricism: the supreme authority of brute fact. But quite the contrary situation is implied by 
Polanyi's striking juxtaposition of the achievements of two "heroes" of scientific history.

. . . Vesalius is praised as a hero of scientific scepticism for boldly rejecting the traditional doctrine that the dividing wall of the heart was pierced by invisible passages; but Harvey is acclaimed for the very opposite reason, namely for boldly assuming the presence of invisible passages connecting the arteries with the veins.

We praise Vesalius for so cleaving to the "testimony of the senses" that he could reject what all since Galen had accepted. But we also praise Harvey (though we condemn Galen) for going beyond the sensory evidence-to a degree denying its authority-in order thereby to "make sense" of the observables. And both Vesalius and Harvey (but not Galen) will ordinarily be claimed for the sacred flock of practitioners of Method.

Certainly scientists are responsive to the authority of facts; presumably in this they are much more responsive than the generality of men. But the absolute authority of facts has been denied-and, we say, most nobly denied-by some who could not otherwise have made the scientific advances for which we honor them. Science is irreducible to a "safe" fealty to "indubitable facts." Often, to advance, men must venture boldly, sustained by nothing stronger than a human faith in human ideas that might well prove wrong-preconceived ideas defended warmly even in the teeth of contrary evidence. We do right to honor Mendeleev who-to sustain his periodic classificationhad to reverse the positions of tellurium and iodine on the optimistic (and quite erroneous) assumption that major errors had been made in determining their atomic weights.

Facts are not enough; empiricism is not enough. Consider the evidence gained by asking: Who advances science? Among the early members of the Royal Society were many who sought to conduct science as an exercise in Baconian empiricism. But the most notable advances in the science of that age were not the work of these faithful cataloguers of facts: de Maistre indeed suggests that generally those who have taken Bacon most seriously have had the poorest success. Might one then argue that what fails here is not Method, but only men holding an imperfect conception of Method? One will then predict that among moderns the older scientists, with a lifetime's experience of Method, should far excel their younger colleagues in the 
production of important discoveries. Well-known facts contravene that prediction. Consider further: important experimental discoveries are made by but one of a hundred men equally trained in Methodequally in command of empirical techniques and of the elementary deductive operations which are all that experimental discovery ordinarily requires of logic. Moreover, the discoverer is often not the most skillful experimentalist nor he most abundantly supplied with experimental devices. Is he simply "lucky"? How very painful to reduce Method to chance!

Du Noüy finds the great discoverer distinguished from the pedestrian fact-collector in this:

The man of science who cannot formulate a hypothesis is only an accountant of phenomena.

An hypothesis is an idea: beyond facts and the logical analysis thereof, the creation of ideas demands imagination. And imaginative capacity I suppose is precisely the faculty weaker in the old than in the young, precisely that so sparsely and unevenly distributed even among men amply trained in Method. Sometimes all too prolific of speculations that harden disastrously into preconceived ideas, imagination remains always the irreplaceable source of the hypotheses that power the successes of empiricism. Bernard writes:

The experimental method, then, cannot give new and fruitful ideas to men who have none; it can serve only to guide the ideas of men who have them, to direct their ideas and to develop them so as to get the best possible results. The idea is a seed; the method is the earth furnishing the conditions in which it may develop, flourish, and give the best of fruit according to its nature. But as only what has been sown in the ground will ever grow in it, so nothing will be developed by the experimental method except the ideas submitted to it. The method itself gives birth to nothing. . . .

. . . Consequently, there can be no method for making discoveries, because philosophic theories can no more give inventive spirit and aptness of mind, to men who do not possess them, than knowledge of the laws of acoustics or optics can give a correct ear or good sight to men deprived of them by nature.

Neither Bernard nor I would for a moment deny that some methodological precepts guide the practice of scientists: my present concern is only to show the vacuousness of the textbook stereotype of Method. 


\section{FINDING THE PROBLEM}

At every stage, effective scientific investigation demands more than Method can supply. Beginning at the very beginning, observe that empirical devices are themselves but rarely the fruits of pure empiricism. The first creation of an NMR spectrometer demanded highly sophisticated theoretical work. But even the humble Dewar flask is an insulating device shaped to its purpose by a theoretical conception of three modes of heat transfer. A similarly abstract pedigree stands behind the startling simplicity of modern chromatographic techniques.

Waive all such considerations. Suppose ourselves presented at the outset with an abundance of empirical tools. We have then to decide "only" how those tools shall be deployed. In a brand new field, random casts may be sufficiently rewarding: we learn what there is to be seen, and even to learn that certain effects are absent is some gain of knowledge. But ultimately we reach the stage of having seen enough, and then we have to ask what is worth observing. Random experiments now become vastly inefficient; from the vast majority of them we learn nothing of value. Thus we come to seek "promising" deployments of our empirical resources. Shall I use my machines to count the pebbles on the beach? Absurd! But why absurd? Because I judge their number irrelevant to any significant problem.

How identify such a problem? Often a highly individualized capacity seems here involved. Others before Newton had seen, and dismissed, the elongation of the prismatic spectrum: to Newton that elongation represented a major problem, one through which he arrived at a new conception of light and color. For millennia men knew of "fanciful" dreams and "trivial" slips of the tongue: in just these Freud saw a substantial problem. So to detect a significant problem, long concealed under pseudo-explanations or in the trivial, demands intuition. Indeed, often it is the discovery and formulation of the problem that demands insight, whereas the solution demands no more than routine mathematical or experimental skill. The identification of a problem both solvable and worth solving is then a matter of prime importance. In this identification "experimental method" cannot help us: what we seek is necessarily prior to our experiments. Nor can we look for support to any Method that denies us the use of preconceived ideas.

Problems can emerge only within contexts of presupposition that 
define the "ordinary" or "natural." Lacking such a context everything and nothing in our experience is a source of wonder and perplexity, everything and nothing is a problem. Given such a context, a problem becomes recognizable as a particular something that seems "unnatural," "incomplete," "irregular," "unsatisfactory." Thus, for example, the retrograde motion of the planets was a problem for the ancients who believed in the perfect uniformity of celestial motions, and not so very long ago the photoelectric effect posed a problem for those who accepted the view of classical electrodynamics. To be sure, if our theoretical preconceptions are wrong, they may lead us to study problems as unrewarding for us as were for them the chemical problems studied by the alchemists. Outlawing preconceived ideas, Method would save us from such error; but then we could not even begin the work of science. The "illicit" context of presupposition discharges the indispensable function of making the difficulty that constitutes a problem, and so first permits the initiation of inquiry.

Not all difficulties pose acceptable problems. Thus I refuse to treat as a problem some "odd" datum I consider likely to prove no more than a trivial experimental error. Making such peremptory dismissal, I draw again on (potentially fallible) theoretical presuppositions. And, if I am to advance any farther, I must now bring into play not only these but some very highly speculative hypotheses as well. Among many possible problems, I can first select one, as a good problem, only as I exercise my imagination to guess its answer. That premonitory intuition, or "educated guess," is then required to support all subsequent stages of inquiry. Thus, attacking my problem, I seek first to collect the already-available data relevant to its solution. Even to begin that collection, I must use a criterion of relevance that finds its sole foundation in my own hypotheses about the form(s) solution to my problem will take. Is the orientation of the tails of comets relevant to the study of light? It first becomes so with the conception of an hypothesis associating that orientation with a "light pressure."

\section{THE DESIGN AND CONDUCT OF EXPERIMENTS}

Proceeding to experiment, I confront the problem of relevance in a new and aggravating form. Abstracting a simple experimental system from the complexity of natural events, what assurance have I that my system in any way "represents" the situation in nature? Is the be- 
havior of balls rolling on an inclined plane relevant to understanding of free fall? Can I hope to gain understanding of the souring of milk by studying a sugared yeast water that contains no milk? How shall I design an artificial system that is relevant, what variables shall I control, what observables shall I note? Before ever I make an experiment on my problem I must bring fully to bear my speculative ideas about its solution(s).

The choice of variables. In an experiment we study a phenomenon in circumstances that are narrowly determined, but never totally defined or definable. In practice we seek to measure and/or control only the relevant variables. To do more is a pointless waste of time; to do less is to aggravate unnecessarily the conceptual (interpretive) problems we will later face. Which are the relevant variables? Presumably just those found relevant in previous studies of closely related phenomena. However, unless we are concerned only with phenomena already thoroughly explored, such relation is only something we hypothesize. Even striking resemblance may here be a wholly insufficient clue: the similar array of colors displayed by rainbow and by peacock tail we find, to our surprise, represent very different phenomena of light. No matter how we are guided by careful observation and long experience, a judgment of "close relation"-and the identification of relevant variables it implies-rest alike on the insecure but essential foundation of those of our preconceptions that dare forecast the results of experiments we have yet to begin.

Can we not entirely bypass so unnervingly uncertain an identification of relevant variables? Rather than dealing with them explicitly, in a controlled experiment, might we not resort instead to use of an experimental control? That is, let us contrive two experimental systems identical in all respects save one. Could we not then conclude that any differences in the results obtained are certainly attributable to the action of the one variable in which the systems differ? Let us then go on to set up many systems, each pair differing only in some one of the many variables conceivably relevant. Could we not then easily establish, by experiment, the identity (and also the specific effect) of each relevant variable?

Indisputably, use of an experimental control gives us an enormously powerful technique. Probably that power is most evident in biological work where-built into the experimental subject itself-a multitude of ill-defined variables elude our control. We cope with 
this major complication by setting up our paired experimental systems with pairs of experimental subjects exactly alike-thus (hopefully, and often actually) arranging to cancel the effects of variables not even known to us as such. Powerful though it is, however, use of an experimental control is no panacea. Literally identical specimens, in systems literally identical in all save one respect, we do not have and cannot secure. We have at most specimens and systems matched in those (relevant) characteristics that, on conceptual grounds, we regard as assuring effective identity. The use of experimental controls makes the problem of relevant variables somewhat less oppressive, but ultimately the problem remains just as real here as when we seek to control the variables explicitly and directly.

Whether in a controlled experiment or in one with experimental controls, we sometimes encounter erratic fluctuations in our results. When these fluctuations substantially exceed our estimate of experimental error, they teach us that we have overlooked some relevant variable(s). But however our data may assist us, neither they nor Method then proclaim the identity of the relevant but uncontrolled variable(s). Always it is we who must go on to guess that. Such guessing is not always easy. Those shrewd investigators Scheele and Priestley long failed to grasp what shortly after was realized by Ingen-Housz: intensity of illumination is a highly relevant variable in the chemical interaction of plants with the atmosphere.

The choice of observables. I cannot control, or even match, all the variables possibly relevant to experimental production of a given phenomenon. But also, among myriad possibilities presented to view, I cannot possibly note all the observables. As early as the 16th century Tycho Brahe-himself neither theoretician nor even experimenter but simply observer-recognized the utter impossibility of "pure" observation. One must have a lead indicating where to look for something worth observing. This essential role of premonitory hypotheses is generalized by Cohen in the following emphatic statement.

Accidental discoveries of which popular histories of science make mention never happen except to those who have previously devoted a great deal of thought to the matter. Observation unillumined by theoretic reason is sterile. . . . Wisdom does not come to those who gape at nature with an empty head. Fruitful observation depends not as Bacon thought upon the absence of bias or anticipatory ideas, but 
rather on a logical multiplication of them so that having many possibilities in mind we are better prepared to direct our attention to what others have never thought of as within the field of possibility.

Guided by an acute surmise founded on the Rutherford-Bohr quantum theory of the nuclear atom, Moseley contrived a particular juxtaposition of equipment: a discharge tube, a series of substances to be put in that tube, a spectrometer containing a photographic plate, etc. These components were assembled for the sole purpose of observing what was in fact found: a simple relation between the position of blackening on the plate and the identity of the substance present in the discharge tube. Something more than a purely empirical genius differentiates the few like Moseley from the many plodders who never make any such important discoveries. Beyond knowing how to measure, he knew also what to measure.

If I err in my selection of relevant variables, a fluctuation of my results may alert me to my error; but if $\mathrm{I}$ err in my choice of $o b$ servables no such indication is vouchsafed me. I simply fail to make headway with my problem-which failure may indeed stimulate, though it cannot supply, the creation of some new hypothesis that directs my attention to hitherto neglected observables. No pat formula for success is to be found in systematic selection of observables quantitatively determinable. Measurement is not co-extensive with science: measurement is but a tool of science and, though often a notably powerful tool, sometimes one entirely inappropriate. Stephen Hales, highly talented scientist and convinced practitioner of the quantitative method-made hundreds of meticulous measurements of the volumes of gases released from various specimens. Never did he sense the much deeper significance of the qualitative differences easily demonstrable in these gases-which included such still "undiscovered" species as oxygen and hydrogen. A more profound insight was won only when, bringing to bear only the very crudest of tests, Priestley and others demonstrated the highly distinctive chemical properties of certain of Hales' specimens of "air."

Today superior theories provide superior bases for choice of significant observables. In any genuinely pioneering study, however, that choice is always and unavoidably hazardous. In Fermi's classic investigation of the interaction of neutrons with uranium, for example, he noted a product he identified as radium. Only the flimsiest experi- 
mental evidence justified this identification, but it was powerfully supported by theoretical considerations. The more extended qualitative examination of the reaction products suggested in 1934, by Ida Noddack, was dismissed as an utterly pointless waste of time and effort. Only five years later did an adequate chemical characterization discover the "radium" to be barium-and this discovery at once evoked the wholly novel concept of nuclear fission.

\section{DRAWING CONCLUSIONS FROM EXPERIMENTS}

Postponing for later consideration the chimeras of methodical induction and crucial experiments, I venture here only a few remarks on the obvious. And surely it is obvious that any harvest of the fruits of empiricism will demand more than logic and empiricism can themselves supply. Like the facts themselves, a general conclusion yields itself only to him who, instructed by his hypotheses, brings to his study a particular query couched in particular conceptual terms. Assume all "errors" properly rejected, all "corrections" properly made: even then what we will be able to find in our data is ordinarily delimited by just what our ideas have prompted us to seek. Possessed by certain theoretic ideas, Dalton found the law of multiple proportions in published data available for several years to all; in precisely similar situations Gay-Lussac discovered his law of combining volumes, Petit and Dulong the law of atomic heats, and Balmer. . . . Hear Poincaré:

The isolated fact attracts all eyes, those of the layman as well as of the scientist. But what the genuine physicist alone knows how to see is the bond which unites many facts whose analogy is profound but hidden. The story of Newton's apple is probably not true, but it is symbolic; let us then speak of it as if it were true. Well then, we must believe that before Newton plenty of men had seen apples fall; not one knew how to conclude anything therefrom. Facts would be sterile were there not minds capable of choosing among them, discerning those behind which something is hidden, and of recognizing what is hiding, ... .

... We are [then] no longer in the presence of a fact but of a law. And upon that day the real discoverer will not be the workman who has patiently built up certain of these combinations; it will be he who brings to light their kinship. The first will have seen merely the crude fact, only the other will have perceived the soul of the fact. Often to fix this kinship it suffices him to make a new word, and this 
word is creative. The history of science furnishes us a multitude of examples familiar to all [e.g., universal gravitation, energy, field].

How materialize "the soul of the fact"? The latent meaning of experimental data appears only with the application of a complex conceptual developer, the formulation of which is irreducible to Method.

\section{THE POWER OF STATISTICAL METHODS}

The elaboration in recent years of refined techniques of statistical analysis may be thought to rehabilitate Method. Teaching us a superior design for empirical studies, and showing us how to get more out of empirical data, the statistical techniques generally simplify the empiricist's tasks. Consider, for example, the use of experimental controls. To investigate one variable we ordinarily seek a pair of systems differing in that variable but matched in all others possibly relevant. When many such variables come in question, we are then committed to the huge effort of preparing great numbers of paired systems matched in all but one variable. Furthermore, hewing to this pattern, we must inevitably fail ever to observe such possible effects as those "synergisms" that materialize only from simultaneous variation of at least two variables. Finally, and most important, we are entirely debarred from certain investigations in which no matching of all the potentially interesting variables is possible: in agricultural studies, for example, often we simply cannot find two sizable plots of land exactly matched in soil, exposure, drainage, and every other significant detail.

The pioneering studies of Fisher and others have taught us how to meet these problems; taught us how, by statistical analysis, to extract meaningful results from the examination of multi-variant systems. With the aid of such analysis the requisite number of trials is sharply reduced, and conclusions otherwise uncertain or wholly inaccessible are brought firmly within our grasp. Recall the co-ordinated simplification and complication we earlier found to accompany the shift from observation to experiment. To win simplification of our conceptual problems we accept the empirical complications of contriving experimental systems that match or control the relevant variables. Now Fisher and his colleagues have forged for us a new conceptual tool of unprecedented power. When strictly controlled $e x$ periment is impossible or inconvenient, this tool permits us to extract 
the knowledge we seek from the superficially more obscure data yielded by what might be described as controlled observation.

The limitation of statistical methods. Undeniably powerful, the statistical tools of thought provide us with notably important new criteria for appraising the relevance or irrelevance of possible variables, and for distinguishing between "error" and the action of some uncontrolled variable. But in neither case does statistical analysis ever identify those variables for us: always these can be suggested only by our own hypotheses. Statistical analysis may reveal in our data some subtle relation we might otherwise overlook. But such analysis never supplants the insight of the investigator: always he must supply the concepts in terms of which alone the analysis can be conducted and its results expressed.

Like specific empirical tools, these explicit conceptual tools are powerful aids to human thought and judgment. But never can they stand in lieu of thought or eliminate the need for judgment. Simplifying judgment at one level, they demand it more heavily at another. Thus, for example, the whole applicability of statistical analysis depends on a judgment that our experimental design suffices to provide results that will constitute a sample truly "random"-and this is no light undertaking when, as is usual, we have access to only a few results. Nothing frees the scientist from an omnipresent. need for such judgments. And generally he makes these decisions rather well, without even thinking of elaborate statistical analyses. Quite clearly he relies then on other than statistical criteria-most clearly when, as often he does, he overrides the statistical indications. Thus he may attach great importance to data statistical analysis would entirely discount: Meyerson, Planck, and many others have commented on the complete inadequacy of the data from which Mayer, Joule, and Colding boldly inferred the invariant equivalence of heat and work. Contrariwise, the scientist may totally reject, as meaningless, data which meet searching tests of statistical adequacy. If Rhine's data on ESP met (or meet) all such tests, still most scientists would reject them out of hand. A case perhaps parallel in point is noted by Polanyi, who refers to

. . . a table of figures published in Nature (146 (1940), p. 620) purporting to show that the days of gestation of different rodents is an integer multiple of the number $\pi, \ldots$ no amount of such evidence could convince us today that this relationship is real . . . 
Statistical considerations alone can never be decisive because scientists always rely more heavily on quite a different criterion of judgment. Cohen points to that criterion while citing yet another case where the statistics are (and rightly, I suppose) simply held irrelevant.

. . . for a number of years the membership in the International Association of Machinists shows a very high correlation ( 86 per cent) with the death rate in the state of Hyderabad. If instances of this sort do not come to our attention more often it is because we do not look for them. We generally look for correlations where we have some reason to suppose that there is a real connection [and we reject correlations whenever we feel that no such reason exists].

But it is we who make the theories and hypotheses which furnish us with "reasons," and we who appraise their adequacy. In such cases our ultimate reliance is patently what indeed it is everywhere. Always in the end we are brought back to human judgments dependent on fallible preconceived ideas.

\section{THE MYTH OF METHOD}

Speaking of self-sufficient Method-scientific, experimental, statistical, or what you will-we speak of a chimera. A Method, seeking to invest the construction of science with an inhuman certainty, must seek a dehumanization of science that ends inevitably by making science humanly impossible. Potential danger then lurks in the myth of Method-danger that working scientists might actually come to take it seriously. In the social sciences credulity of this myth exacts a heavy toll-as on occasion it does even in psychology, presumably itself a natural science.

Clipping the wings of inspiration, strictures of Method must cripple the flight of science. Born writes:

I believe that there is no philosophical highroad in science, with epistemological signposts. No, we are in a jungle and find our way by trial and error, building our road behind us as we proceed. We do not find signposts at crossroads, but our own scouts erect them, to help the rest.

Methodological scruples endanger the bold, lawless enterprise of scouts, which has made science what it is today-which will again be required to make it all it hopes to become. However shockingly, awk- 
ward facts may on occasion be wisely ignored; and, in making a beginning, irreducibly vague concepts prove often immeasurably superior to logically impeccable categories that presuppose all need for investigation is at an end. An obsessive concern with methodological chastity does not often eventuate in scientific fecundity.

Great scientists practice not Method but success. Determined to get on with his difficult job, however best he can, the working scientist, in Einstein's words,

. . . must appear to the systematic epistemologist as a type of unscrupulous opportunist: he appears as realist insofar as he seeks to describe a world independent of the acts of perception; as idealist insofar as he looks upon the concepts and theories as the free inventions of the human spirit (not logically derivable from what is empirically given); as positivist insofar as he considers his concepts and theories justified only to the extent to which they furnish a logical representation of relations among sensory experiences. He may even appear as Platonist or Pythagorean insofar as he considers the viewpoint of logical simplicity as an indispensable and effective tool of his research.

The growth of the natural sciences utterly defies reduction to Method, to the constellation empiricism-fact-logic. I have distinguished the empirical and conceptual lobes of the heuristic apparatus to facilitate discussion, and because between them there is all the distinctness of facts from ideas. But ultimately we find the facts inseparable from the ("subjective") ideas with which we seek, recognize, express, and appraise the relevance of ("objective") facts. Then strikes the hour to move on beyond empiricism: however large it looms in the history of science, Langer accurately remarks the something else that looms still larger.

The limits of thought are not so much set from outside, by the fulness or poverty of experiences that meet the mind, as from within, by the power of conception, the wealth of formulative notions with which the mind meets experiences. . . . A new idea is a light that illuminates presences which simply had no form for us before the light fell on them. 


\section{CHAPTER VII}

\section{The Principles of Science}

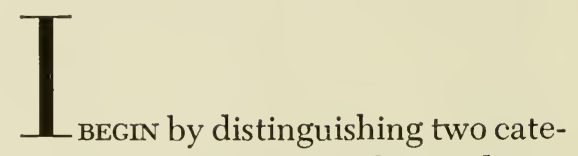

gories of principles: a small group of regulative principles, with implications for the conduct of science; and a much larger group of substantive principles constituting "established knowledge" for the scientists of an age. The substantive principles supply a point of departure for scientific thought; the regulative principles sketch the goals of such thought and, very tentatively, routes thereto. Thus, in effect, the regulative principles assert something about the optimal construction of science, whereas the substantive principles assert something about the actual construction of the world. To be sure, this distinction is not perfectly clean-cut. If we accept a particular regulative principle, and seek to build scientific knowledge in certain ways, we do so only because we also accept a certain conception of the object of knowledge: the construction of the world. Nonetheless I will maintain what seems to me this useful distinction between substantive and regulative principles: some haziness of classification must, I think, be tolerated when we have to deal with the fundamental principles constituting that integral context of science of which Nicod says:

It stays in the shadow and yet guides us to the light; we know how to use it, but we do not know how to analyze it.

Beginning with the regulative principles, observe that they have the function (but not the status) of Kant's a priori ideas. Kant suggests that the multifarious data of human experience are in them- 
selves formless, inchoate, inapprehensible-that they can be "taken in" only as the human mind imposes on them its own patterns of comprehension. To the contemplation of experience we thus bring our own ways of seeing: $e . g$., we "see" an ordered world close bound in chains of causal connection. Such ways of seeing or knowing are not imposed upon us by our experience. On the contrary, they are presuppositions of human experience. These patterns of thought constitute the co-ordinate system in which we view and measure our experience, the ledger form in which we enter our findings as we "take stock" of that experience.

Kant felt that men must always view the world in terms of certain unchanging forms innate to their very humanity: for him these forms were a priori absolutely. Contrariwise, I will regard the regulative principles of science as a priori only relatively. The efflorescence of human minds in some particular age and clime, they remain forever susceptible to change under the coercive pressure of an ever-widening human experience. To be sure, we are very slow to admit any such coercion. The regulative principles are the most essential of the heuristic tools we bring to bear on our experience. Perforce, we make the tool harder than that on which it is to work. But it is not infinitely harder: slowly the tool is itself reshaped by the uses to which it is put.

The status of the regulative principles defies simple categorization. Indubitable in the short term, none of them is today entertained in quite the form it had some centuries ago, or will probably have some centuries hence. Metaphysical in that they go far beyond all possibility of empirical demonstration, yet are they different from the perennially disputed abstractions of philosophy and theology: though metaphysical, they elicit the general consensus of scientists who find them justified by and in experience. Maintained with deep conviction, they are appraised by pragmatic criteria which are, of their very nature, provisional. Normally implicit, and never clearly formulated as such, these principles may seem instinctive feelings (reflection of the scientific instinct of an age); but they are also judgments (no less so because they may be unconscious judgments) in that they change as new evidence comes within our experience. Cosmologic, as covert expressions of a conception of the world, their primary function is methodologic, though they constitute no concrete Method. Perhaps they are best described, as some of them were by Newton, as "Rules of Reasoning in [Natural] Philosophy." They are 
"rules of the road," presupposing certain characteristics of earthly roads and human drivers, but they do not teach us to drive.

\section{The Existence of a Real World}

For such as Occam, Berkeley, and Eddington, the reality of an external world poses a major problem; but for others, like Bronowski, that world is simply given.

We do not construct the world from our experiences; we are aware of the world in our experiences.

In exactly the same vein Brain writes:

We do not need to ask how we become aware of things ouside ourselves because it is with that awareness that we begin.

Whether it is a something postulated or a something given, an existing real world is assuredly a something taken for granted by scientists. To what was said of this principle in Chapter II I have now to add one note of emphasis. I seek only to state, and indicate the application of, this principle and all other regulative principles treated hereafter. Never do I seek to justify them: taken as methodological principles, they are amply accredited by the demonstrable triumphs of the science that acknowledges them. I recognize no need (or way?) to justify them on any other grounds.

\section{The Principle of Intelligibility}

The real world having been constituted a "something," the principle of intelligibility asserts man's capacity-perhaps even his obligationto understand that something. No Kantian necessity of thought, the first full conception of man's capacity for understanding appeared only with the coming of the Greeks. That the search for understanding is in any sense an obligation is a much later conception, even today rarely acknowledged outside the community of scholars. In some part inspirational, the principle of intelligibility has profound methodological implications.

\section{FOUR METHODOLOGICAL COROLLARIES}

Seeking to understand the world, we are driven beyond colligative relations-beyond "natural laws" the principle of intelligibility as- 
sumes both existent and discoverable-in quest of explanation of those laws. This quest seeks consummation in the construction of postulational system(s) in which the laws are demonstrated to be necessary consequences of theoretical premises we accept. But nowhaving learned to reject, as delusive, the hope that theoretical premises are, or can be made, self-evident-we cannot but recognize that always our explanations are incomplete. Hall attributes to Galileo and Newton the opinion that:

The explanation of phenomena at one level is the description of phenomena at a more fundamental level, . . .

Complete understanding then fails by the margin of those theoretical premises which are stipulated, perhaps "described," but certainly not themselves explained or explicable for so long as they remain our ultimate premises.

Parsimony. Resolved to maximize our understanding, we find ourselves committed to a highly characteristic effort to minimize the number of theoretical premises required for explanation. Einstein speaks of:

. . . the grand aim of all science, which is to cover the greatest possible number of empirical facts by logical deductions from the smallest possible number of hypotheses or axioms.

Some centuries earlier Newton had expressed the same "grand aim" in the first of his Rules of Reasoning:

We are to admit no more causes of natural things than such as are both true and sufficient to explain their appearances.

This is in turn the millennially remote echo of the "master of those who know," writing when the principle of intelligibility was in its infancy.

That is done in vain by many means which may equally well be done with fewer.

Major differences of attitude and meaning presumably underlie these statements. Quite clearly, however, acceptance of the principle of intelligibility has always provoked, must always provoke, a continuing endeavor to devise theories of ever-increasing comprehensiveness founded on ever-narrower postulational foundations. Of many specific manifestations of this endeavor, a particularly signifi- 
cant example is the enduring effort to construe the variety of the world in terms of a very few "elements." That attempt begins with Thales, the earliest of the Ionian philosopher-scientists; and the modern physicist, with his "fundamental particles," presses on in the same endeavor. Generalizing "elements" to include intangibles like force and energy, we find in Newton's Principia an expression of the same striving, for he says:

... the whole burden of [natural] philosophy seems to consist in this-from the phenomena of motions to investigate the forces of nature, and then from these forces [small in number and taken as axioms] to demonstrate the other phenomena.

Sambursky emphasizes the absolute continuity of the endeavor while citing yet another example of it:

The laws of conservation are now so essential a part of science that it is hardly conceivable that we should be able to do without them or that science should take any form which does not allow for the formulation of such laws. The possibility of such formulation is implied in the premise which the Milesian School considered self-evident: that nature is capable of a rational explanation which reduces the number of variables and replaces some of them by constant quantities independent of time or of the particular form of a given process.

Penury. Each "quality" imputed to a premised entity figures as an additional postulate. Our desire for parsimony of postulates thus evokes a search for theoretical posits having the slenderest possible qualitative endowment. But this last quest derives more fundamentally and directly from the principle of intelligibility. Dingle makes it a principle of rational thought that:

. . . an entity which is postulated to explain a general property of observable entities must necessarily lack that property.

We reject theories that offer no more than a first step in a potentially infinite regress. We find no sense of explanation in the attribution of the wetness of water to the wetness of water molecules, or of the hardness of iron to the hardness of iron atoms. As well, we think, explain the soporific action of opium by a dormative virtue inherent in it. Today Heisenberg remarks that:

It is impossible to explain . . q qualities of matter except by tracing them back to the behavior of entities which themselves no longer 
possess these qualities. If atoms are really to explain the origin of color and smell of visible material bodies, then they cannot possess properties like color and smell.

But close to the beginning of the era of modern science we find the same insight in Newton, though not in all of his contemporaries (or ours). Having in mind a further question (What is responsible for the cohesive strength of an "atomic hook"?), he remarks:

The parts of all homogeneal hard bodies which fully touch one another, stick together very strongly. And for explaining how this may be, some have invented hooked atoms, which is begging the question; ...

The qualities for us "explained" by a theory can only be those not imputed to the primary entities of that theory. The greater their penury-the fewer the qualities they possess-the more numerous become the qualities rendered potentially explicable by the theory. Any quality so explained is then shown comprehensibly emergent (in a sense presently to be examined) from the deportment in a context of entities lacking precisely that quality.

Evolutionary theories have in this respect immense explanatory appeal. Carrying this line of thought to its extreme, we set out from Gamow's concept of a qualitatively undifferentiated super-hot superdense plasma in which, some billions of years ago, a nuclear explosion on a cosmic scale was followed by a physical evolution of elements. That development he supposes followed (and completed) by an astronomical evolution of galaxies, suns, planets, etc.; and that in turn by such a geological evolution as has produced the varied topography of our planet. Instructed by Oparin and others, we may then conceive a chemical evolution, yielding moderately complex molecules, and carrying on through some $\operatorname{step}(\mathrm{s})$ as yet unknown to the production of one or more rudimentary forms of life. By a further biological evolution we may, with Darwin, suppose higher animals like ourselves produced, then to undergo a sociologic evolution, and so on and on.

In this chain of theories many links are at present little more than speculations. But such an evolutionary development does propose a potentially acceptable scientific explanation-conspicuously characterized by the continual emergence of diverse qualities and complexity from systems having fewer qualities and less complexity. 
Complexity and diversity are so explained as they never can be by the theological view of original creation, in detail, of all the universe and its denizens. That view requires postulation of a god unimaginably more complex than the universe to be explained. The evolutionary sequence, contrariwise, traces the infinitely variegated world of our experience back to ylem-the undifferentiated plasma with most meagre of qualitative endowments.

Ylem itself is describable, but certainly not explained. We might postulate its creation by a god-himself self-created that the regress may be cut off. As well invest ylem with power of self-creation! In the current development of science, ylem is simply that posit which is for us ultimate. Recognizing that, I recognize no failure. In ylem the quests for parsimony and penury are notably gratified. Beyond this, ylem has also the incomparable virtue of clarity. Though unexplained, ylem can be described as a divine creator cannot be. We seek explanation through deduction of laws from premises: obviously we must seek premises clearly enough describable to support straightforward derivation of conclusions.

Clarity. Whatever appearance of clarity they may make, the premises of some theories prove, on closer inspection, far too obscure to reason from, far too exiguous to support any genuine explanation. Of this kind, Bernard remarks, is the premise that characterizes all vitalist theories.

Life is nothing but a word which means ignorance, and when we characterize a phenomenon as vital, it amounts to saying that we do not know its immediate cause or its conditions. Science should always explain obscurity and complexity by clearer and simpler ideas. Now since nothing is more obscure, life can never explain anything.

Scientists find notably repugnant all theories of this vitalist stampand correspondingly attractive those mathematically constructed theories that seem to turn on only the simplest of ideal entities standing to each other in the clearest of relations. Most often the posits are eminently penurious and, beyond that, in such theories we seem to achieve the very utmost of postulational parsimony. From the small group of premised relations a veritable myriad of derivative theorems seem unequivocally and effortlessly produced. The superb power of correlation so displayed awakes in such as Newton a compelling sense of aesthetic splendor. 
. . . it is the glory of geometry that from those ferv principles, brought from without, it is able to produce so many things.

Does this sense of glory have another, deeper root? Beyond the exemplary clarity they offer, do mathematical representations of the world have for us another antipodal appeal rooted in an obscure mysticism holding number to be the essence of things? However paradoxically, this mysticism is close kin to the principle of intelligibility-as when the Pythagorean philosopher Philolaus argues that:

. . Number, fitting all things into the soul through sense-perception, makes them recognizable and comparable with one another. . . . Actually, everything that can be known has a Number; for it is impossible to grasp anything with the mind or to recognize it without this.

Even numerical laws (as distinct from theories) then take on a special meaningfulness. Koestler remarks that:

The Pythagorean discovery that the pitch of a note depends on the length of the string which produces it, and that concordant intervals in the scale are produced by simple numerical ratios (2:1 octave, $3: 2$ fifth, $4: 3$ fourth, etc.), was epoch-making: it was the first reduction of quality to quantity, . . .

. . . The gross strings of the lyre are recognized to be of subordinate importance; they can be made of different materials, in various thicknesses and lengths, so long as the proportions are preserved: what produces the music are the ratios, the numbers, the pattern of the scale. Numbers are eternal while everything else is perishable; they are of the nature not of matter, but of mind; they permit mental operations of the most surprising and delightful kind without reference to the coarse external world of the senses-which is how the divine mind must be supposed to operate.

Pythagorean mysticism can be deleterious, for example in encouraging neglect of the "coarse external world of the senses." But it also inspires an extraordinarily fruitful effort to construe phenomena of nature in terms of simple mathematical "harmonies," both numerical and geometric. That inspiration informs the work of Archimedes in the ancient world, and plays a decisive role in the rebirth of science in the modern world at the hands of such as Copernicus, Kepler, and Galileo. For such men the discovery of mathematical harmony in natural phenomena was in itself an explanation of those phenomena, 
to be prized beyond all other possibilities of explanation. In our own age this spirit still moved in Einstein, who expressed a very deepseated conviction in writing that:

I feel sure that pure mathematical construction allows us to discover the concepts, and the laws connecting them, which supply the key to the understanding of natural phenomena. . . . In a certain sense, therefore, I hold it to be true that pure thought can comprehend reality, as the ancients dreamed.

Those who share this conviction find explanation in the mathematical form of a physical theory. Of course, not all scientists share this conviction, and not all accepted scientific theories have this form. Moreover, we shall find in the next chapter that a theory cannot be purely mathematical if it is to function at all as a physical theory. We must be able to find in its abstract postulates something more than the epitome of clarity: in them we must find also some sense of analogy.

Analogy. "Explain" laws by derivations from theoretical postulates unexplained and inexplicable in the context of the theory they constitute? To wield the theory effectively, clearly we must somehow learn to grasp its postulates-and this we always seek to do by relating them analogically to things or situations that serve us as "models." Thus, Bridgman observes,

. . . the model is a useful and indeed unescapable tool of thought, in that it enables us to think about the unfamiliar in terms of the familiar.

The Ionians perforce sought models outside the science they were first to create-and found them everyuhere, e.g., in the action of the winnower's sieve, and the operation of felting. However, by the middle of the 19th century, one particular privileged class of scientific models was established by the rise of classical mechanics. The major scientific synthesis extant, it was thoroughly familiar, apparently simple, and superbly competent to give a convincing account of just those purely mechanical systems of which man had had the longest and widest experience, and now felt the deepest understanding. A Kelvin might then understandably assert:

I never satisfy myself until I can make a mechanical model of a thing. If I can make a mechanical model I can understand it. As long as I cannot make a mechanical model all the way through I 
cannot understand; and that is why I cannot get the electromagnetic theory. . . . I want to understand light as well as I can without introducing things that we understand even less of.

Even today many still prefer mechanical models: of all models they are the most directly linked with both familiar everyday experience and the events actually observed in the laboratory. But today few indeed adhere to Kelvin's opinion that only mechanical models are acceptable. Thoroughly justified for so long as mechanics was the one established scientific system, his conception becomes altogether too narrow as soon as there appear other well developed sciences with which we feel enough at ease to frame our models on them. Today, for example, we happily found "field theories" on a model supplied by precisely that classical electrodynamics Kelvin still found unintelligible in itself. A century ago men dealt only in mechanical models formulated explicitly. Today we deploy a diversified array of physical models that, in some cases, may figure only implicitly in a theory constituted by little more than a set of equations. However vague, distant, or incomplete may be the analogy-however deeply hidden in the mathematical formalism may be the physical model-always they are indispensable to our grasp of the theory. But, having forged on beyond mechanical models, we now recognize our capacity to pass beyond any predetermined set of "acceptable" models.

\section{CONGRUITY}

Accepting the principle of intelligibility, we set ourselves to understand a world we assume humanly understandable. We may bolster our courage with the assertion that "nature is simple," so that even feeble human reason may comprehend it. The effect is the same if we assert the complexity of nature and the vast power of human reason. Actually of course we need, and can, assert neither simplicity nor complexity of world and mind: our basic assumption is the congruity of world and mind. For the earliest scientists the assumption of congruity was justifiable only as, for the religious, Pascal's wager might justify faith in his God. Today that assumption is buttressed by a progress of science Einstein holds ample to demonstrate that nature is humanly comprehensible.

One may say "the eternal mystery of the world is its comprehensibility." . . . 
In speaking here concerning "comprehensibility," the expression is used in its most modest sense. It implies: the production of some sort of order among sense impressions, this order being produced by the creation of general concepts, relations between these concepts, and by [various determinate] relations between the concepts and sense experience. . . . It is in this sense that the world of our sense experiences is comprehensible. The fact that it is comprehensible is a miracle.

The "miracle" can, of course, be taken in various ways. Edelstein writes in a somewhat Kantian vein that:

On the basis of the hypothesis that the world can be understood by reason some men in Ionia in the early 6 th century created a world that reason understands.

Here congruence would arise from "construction" of the world by mind. Galileo thought differently, rejecting the view that:

. . . Nature had first made men's brains and then disposed all things in conformity to the capacity of their intellects. But I incline rather to think that Nature first made the things themselves, as she best liked, and afterwards framed the reason of man capable of conceiving (though not without great pains) some part of her secrets.

Today we incline very strongly to Galileo's opinion, thinking to have found, in the theory of evolution by natural selection, a mechanism competent so to have "framed the reason of man." By that mechanism Einstein's miracle is toppled into a class with so many others that once loomed large in the argument from design. Hear Sherrington:

Our stock is the vertebrate stock; our body is the vertebrate body; our mind is the vertebrate mind. If the vertebrates be a product of the planet, our mind is a product of the planet. Its activities and proclivities declare it so. Its senses each and all gear into the ways and means of our planet which is its planet. They are adapted to it, as a fish's body to water. . . . Ours is an earthly mind which fits our earthly body. It produces percepts of earthly things from an earthly viewpoint. It helps the besouled body to deal with terrestrial things, thereby to live. Our mind constructs "time" and its time's rate is that of its besouled body's terrestrial habitat; although it, not unnaturally, has supposed it to be an universal and absolute Time. The last preceding turn of its planet is its "yesterday" and the next expected turn will be its "tomorrow," . . .

... We are, in biological phrase, reactions. The situation creates 
the life which fits it. The dry land created the feet which walk it. Our situation has created the mind which deals with it. It is an earthly situation. Along with the sea it has created in us the wonder of the sea. The situation engenders the reaction to it. If the agent is terrestrial and the reaction is terrestrial is not the medium of the reaction terrestrial? The medium is the mind.

Simplicity sigillum veri. Newton still assumed some absolute simplicity of the world when he wrote:

. . . Nature does nothing in vain, and more is in vain when less will serve; for Nature is pleased with simplicity, and affects not the pomp of superfluous causes.

But even when we posit no more than congruity of world and mind, we must continue to impute special status to what we judge "simple." If our scrutiny of nature seems to show any "superfluous causes," we then come quite naturally to suppose our examination defective or incomplete. Thus in biology each differentiated tissue or organic manifestation is confidently assumed to have, if not a purpose, then a function we set ourselves to discover. Von Frisch's entire epochmaking study of bees took departure, he tells us, from his flat rejection of the claim that the colors of flowers have no biological significance. Teleology, today not longer acceptable as an argument, conserves its power as heuristic guide. Von Bruecke, indeed, draws the general conclusion that, although teleology is a lady with whom the contemporary biologist is reluctant to be seen in public, he finds it impossible to live without her.

A number of more "respectable" implications attach to our conviction that we must find nature simple when we see her truly. Consider for example Jeffreys' comment:

The actual behavior of physicists in always choosing the simplest law that fits the observations therefore corresponds exactly to what would be expected . . . if they considered the simplest law as having the greatest prior probability.

Finding difficulty with Jeffreys' whole concept of prior probability, I regard the characteristic behavior he so accurately reports as just one more direct consequence of our acceptance of the principle of intelligibility. The simple law is just that species of law we know how to explain by theoretical derivation. Supposing that all genuine laws are. 
so explicable, we are then at once predisposed to see the simplest law as that most probably genuine. However this may be, the same strain of thought active in determining the laws we seek, and choose, seems also active when laws fail unexpectedly. Always we seek to understand such failure in terms of one or a very few "complicating factors."

Of our theories, as of our laws, we hold simplicity the mark of truth. But here the situation becomes very difficult. Using this touchstone for the assay of scientific theories, all too often we obtain highly equivocal results. Retrospectively we see that simplicity cannot supply a decisive criterion of judgment, for a perfectly obvious reason: there is complexity to the whole idea of simplicity. Simplicities differ in kind as well as in degree. Perhaps we may distinguish the "simplicity" of great explanatory appeal (e.g., the strong sense of analogy aroused by a convincing model) from the "simplicity" of great correlative power (e.g. "the glory of geometry"). Probably, however, the two categories are seldom wholly distinct: $e . g$., is the "glory" ever without explanatory appeal? And, certainly, in judging simplicity we always face the problem of somehow weighing together (if only against each other) two qualitatively different species of simplicity-within which lurk still further orders of complexity I examine hereafter.

In time we learn to attach greater weight to one type of simplicity than to another. But then, all the more clearly, as sigillum veri, simplicity becomes a non-absolute criterion of judgment-an unknown function of a whole shifting climate of opinion, both scientific and cosmologic. Nevertheless, however difficult and insecure may be our judgment, and however insufficient in the short term may be this criterion, no scientist ever doubts that simplicity is a criterion for the judgment of theories. Given the principle of intelligibility, that could not be doubted. And, however our ideas of simplicity change in time, we have consistently set a high value on that particular species of simplicity we call "sameness." If for no other reason, we would prize this just because we are so certain that, awaiting our discovery, there is sameness in nature. Acceptance of the principle of intelligibility renders inadmissible any doubt on this point. Consider: in humans knowledge presupposes learning, and learning necessarily presupposes in nature some degree of sameness in time, place, and species. Human knowledge thus presupposes precisely that uniformity Mill regarded as the ultimate major premise of all inductions. But now the principle of intelligibility asserts human capacity for knowledge of 
nature, and so has as corollary Mill's ultimate premise. So important and complex is this corollary that I will treat it as a separate principle.

\section{The Principle of Continuity}

Heraclitus found in nature no continuity beyond that of absolute flux, continuous nonrecurrence. And even of those Greeks who descried a stable order in nature, Sambursky finds few who conceived the extent of that order as Anaxagoras did.

Anaxagoras' astronomical hypotheses are throughout dominated by a "terrestrial" approach which makes no distinction between phenomena "there" in the sky and those "here" on the earth, and gives a purely physical evaluation of astronomical data and their possible causes. The heavenly bodies are nothing more than flaming stones; "the sun is larger than the Peloponnesus"-what uninhibited freedom of thought is revealed by this comparison of the mightiest of the celestial bodies, apotheosized by the deep-rooted irrational beliefs of mythology, with a geographical object, a part of the inhabited earth!

Some two millennia were yet to pass before this view would find its match, and consummation, in Isaac Newton. Extending his concepts to earth and cosmos (and hypothetical microcosm) alike, Newton acts always on this premise:

This is the quality of all bodies within the reach of our experiments; and therefore . . . to be affirmed of all bodies whatsoever.

To this conception of continuity Newton attached so much importance that he makes of it a second Rule of Reasoning, corollary to the first. Conceiving nature as "wont to be simple and always consonant to itself," Newton takes it as a matter of principle that:

Therefore to the same natural effects we must, as far as possible, assign the same causes. As to respiration in a man and in a beast; the descent of stones in Europe and in America; the light of our culinary fire and of the sun; the reflection of light in the earth, and in the planets.

Newton's successors applaud, and act upon, this principle-holding its soundness attested by Newton's successes and their own. By those successes (and co-ordinate failures) we have, indeed, been taught a revulsion from uniqueness and abruptness that carries us far beyond 
the idea of sameness in time and place. Always now we would conceive nature as a continuum in which gradualism is the predominant (though not invariable) order of things. That conception is brought to bear, as earlier indicated, even in the elementary operation of fitting to our experimental "points" lines along which we interpolate and extrapolate. Active here at the very instant we make a first appreciation of our data, the same conception remains active also right through to the highest levels of theoretical analysis.

\section{CONTINUITY AND EXPLANATION}

The connection of intelligibility and continuity, though asymmetric, is reciprocal. We seek a species of knowledge that presupposes continuity, and with us the recognition of continuity passes as knowledge. The abrupt outbreak of flames from a pile of oily rags constitutes for us a problem. To it we find a satisfactory solution in the conception (and detection) of a slow process of oxidation, autoaccelerative as it produces a continuous gradual rise of temperature. That rise simply culminates in the appearance of flames, and these signify only a more rapid progress of an oxidative process we conceive different only in degree and not in kind from that taking place earlier. Passing from the trivial to the cosmic, observe that no small part of the appeal of a cosmogony of continuous creation derives from the analogous suppression of a unique (and unfathomable) "beginning."

Forever seeking continuity where continuity is not apparent, we show ourselves still responsive to what was, even in the infancy of science, taken as of principle by Democritus:

Nothing can be created out of nothing, nor can it [something, presumably] be destroyed and returned to nothing.

If a something seems to disappear into nothing, understanding or explanation requires the conception of a posterior something into which it has been metamorphosed. If a something seems to appear out of nothing, understanding or explanation requires the conception (and, if possible, detection) of an anterior something responsible for its production. The sudden appearance of a positron-electron pair perplexes us until we can responsibly hypothesize the antecedent presence of an unperceived gamma ray from which they have been produced. Likewise we refuse to suppose that light is generated only to vanish at its source and subsequently to reappear at its destination. 
Instead we constitute light a "something" which travels from source to destination, thus achieving continuity of connection in space as well as continuity of existence in time.

Some half-century ago quantum phenomena began increasingly to breach the conception of continuity so long taken for granted. Secure in that conception, we reject out of hand the supposition of some early societies that the sun is annihilated at dusk and reborn at dawn; but Bohr's conception of "quantum jumps" may be held to imply that an electron disappears here and reappears there without any intermediary "existence." As a matter of record this denial of continuity proved highly offensive to many physicists of the early 20th century, and even today a few are deeply disturbed by what Schrödinger called "the nightmare that physical events consist in continual sequences of little fits and jerks." That this should seem a "nightmare" is testimony to the strength of the abiding human concern with continuity. Indeed this nightmare parallels (perhaps even in futility?) a problem that, in a very different age, agitated the scholastics: when angels move from place to place, do they pass through the intermediate space? In any case, whatever the ultimate outcome of attempts to dispel Schrödinger's nightmare, quantum mechanics still preserves absolutely intact those major elements of continuity embodied in the various conservation laws.

Energy. For Anaximenes "motion is from eternity." Today "kinetic energy" has become one measure and expression of the quantity of motion. But clearly kinetic energy is not constant even for one single oscillation of a simple pendulum. To maintain constancy of "energy" we then introduce a term representing "potential energy," hypothesizing that the sum (but not the distribution) of energies remains constant. Of course, kinetic and mechanical potential energies are insufficient to maintain the constancy we seek in all the cases with which ultimately we must deal. In the 19th century, conservation of energy required the postulation of multiple species of interconvertible energies-thermal, chemical, electric, magnetic, radiant, etc. At the beginning of the 20th century, the apparently limitless evolution of heat by the then-newly-discovered radioactive elements posed a problem resolved only with the conception of an interconvertibility of mass and energy-mass becoming itself a species of energy. Little more than a quarter of a century ago, what seemed a genuine crisis was averted only with the postulation of the neutrino. A purely ad 
hoc contrivance, this "particle" was purposively designed to be the undetected, and then undetectable, vehicle for the conveyance of energy from experimental systems in which the conservation law (and others of its kind) apparently fail.

Some two centuries ago, close to the beginning of this very complex development, its leitmotif was voiced by Bernoulli in a statement which echoes Democritus:

To try to demonstrate this law would be to obscure it. Indeed, everyone regards this as an incontestable axiom, that an efficient cause cannot perish, either as a whole or in part, without producing an effect equal to its loss.

That axiom being held incontestable, we have throughout pursued an elusive constancy in the absolute conviction that some such constancy exists. Thus, as Meyerson emphasizes, we have perfectly consistently multiplied species of energies if and as required for constancy of "energy."

The quest for continuity we have found so imperious, and so highly profitable, here yields a conservation principle like all other conservation principles. By dint of conceptual invention and empirical discovery, at last we reveal-underlying even the most drastic changeone perfect invariance. Invariance in hand, we now sense within reach that incomparably satisfying absolute continuity we call identity. But here, alas, what remains identical is an exceedingly abstract "essence" difficult to conceive and, if Poincaré is right, impossible to define.

In each particular case it is clearly seen what energy is and at least a provisional definition of it can be given; but it is impossible to find a general definition for it.

If we try to enunciate the [conservation] principle in all its generality and apply it to the universe, we see it vanish, so to speak, and nothing is left but this: There is something which remains constant.

The conservation principle is then an incomplete consummation of the quest for absolute identity in nature, which, however, we can pursue in another direction. This particular pursuit is indeed perhaps the most ancient and compelling effort spawned by the principle of continuity, and also one to which modern science owes much of its cachet. We sacrifice continuity in space to gain a perfectly genuine identity in time. 
Atomism. Of the appearances of change and permanence in our world, Heraclitus dismisses permanence as illusory, while Parmenides so dismisses change. Wishing to dismiss neither, we forever seek to found on permanence-on some element(s) of timeless identity we willingly take for granted-our conceptualization of whatever is observed to change with time. Change in the medium-sized world of our experience we seek then to derive from permanence in either macrocosm or microcosm, respectively enfolding or underlying the everyday world. Initially the first possibility may seem more plausible: the cycles of tides and seasons are obviously correlated with cycles of moon and sun respectively. And astrology seeks the origin of most or all terrestrial change in the macrocosm, in "influences" arising from the shifting juxtapositions of eternal bodies pursuing their eternally appointed rounds. Mutability then derives from the immutable, the "local" motions and changing dispositions of which are not for us inconceivable. Only the millennial incompetence of astrology finally discredits this possibility.

The second alternative is the one Leucippus and Democritus explored. Again qualitative change is construed in terms of local displacements of the unchanging-here in a hypothetical microcosm, the world of atoms. Thus, in Newton's phrasing,

... the changes of corporeal things are to be placed only in the various separations and new associations and motions of these permanent particles; . . .

Slightly earlier, but some two millennia after Parmenides, Boyle had maintained that:

If an angel himself should work a real change in the [qualitative] nature of a body, 'tis scarce conceivable to men, how he could do it, without the assistance of local motion.

Two centuries later du Bois-Reymond still saw things the same way:

The scientific cognition of nature . . . is the reduction of changes in the physical world to the motions of atoms in accordance with central forces independent of time . . . It is a fact of psychological experience that our yearning for causal understanding is, for the time, well satisfied whenever we succeed in making that reduction.

Beyond conceptual attractiveness, the corpuscular view today offers proven power. In all chemistry qualitative change is conceived in 
terms of alterations in the (molecular) groupings of unaltered (atomic) particles, now including electrons and nucleons. In biology the fluctuating appearance of heritable traits is similarly referred back to the fluctuating combinations of "atomic" genes (and, today, to the sequences of "atomic" base groups in DNA molecules). And so on and on.

The invariance expressed in a conservation law is, in very strong form, just such a recognition of continuity as passes with us as knowledge. And then, quite naturally, recognition of what invariance demands will count with us as explanation. So it is that we couple the atomic theory with conservation laws to explain the occurrence (or nonoccurrence) of certain qualitative appearances, corresponding to particular states or structural arrangements. Leibniz considered atomism a theory flagrantly, and unacceptably, in violation of the dominant concept of continuity. But we find no irremediable incompatability between the discreteness of atoms and the element of continuity so prominent in the conservation principles. Continuity and discreteness are, after all, only imaginary conceptual polarities never wholly separated in practice. We use them to designate modes of thought (e.g., particle theories and field theories) in which one or the other polarity seems dominant. The separation of the polarities may then be highlighted by occasional spectacular conflicts of such theories-e.g., the ancient strife of atomist with Stoic, the modern strife of morphologist with neo-Darwinian-but no scientific theory fails to embody and unite, in some degree, both polarities.

Beginning with (common-sense) concepts of discrete objects, scientists may go on multiplying "things": galaxies, stars, planets, atoms, electrons, photons, etc. But ultimately they seek somehow to re-establish the spatial continuity these concepts will seem to set at nought. Thus, for example, having begun by conceiving a sharp boundary between stationary pipe and fluid everywhere flowing at some bulk velocity, ultimately we conceive (and find) a gradient of flow velocities bridging the gap between a maximum value, along the axis of the pipe, and a minimum, next to its walls. Analogous dissipations of abrupt discontinuities occur at the furthest reach of scientific thought.

Newton first attacks the problem of celestial dynamics with a concept of mutually attracting discrete bodies. Rebelling against the idea of action at a distance, ultimately we seek to re-establish continuity 
by filling interplanetary space with a "field." This endeavor, as Sambursky observes, reaches its supreme expression in Einstein's general theory of relativity, where

. . . a physical point is simply a singularity in the "metric field" which surrounds it. Again, this field is not at all an empty space, but a kind of emanation of the matter in it, just as matter is a kind of "materialization" of the field.

At the very opposite end of the scale of magnitudes, we find in modern microphysics an entirely analogous reduction of what once appeared an absolute dichotomy. To the discrete corpuscular "electron" of the 19th century we now impute also some of the characteristics of a continuous wave and, conversely, to the purely undulatory "light" of the 19th century we now impute some of the properties of a corpuscle. Again there is a marriage of continuity and discontinuity and, though quantum mechanics has so far provided only a shotgun wedding, the union has been fruitful and enduring.

\section{DISSOLUBILITY AND SUPERPOSITION}

Superposability, the possibility of reconstituting wholes from parts, I regard as corollary to the principle of continuity. Dissolubility, the separability of parts from wholes, is taken as a principle even by common sense. Already examined from various aspects in Chapters II, III, and V, the principle of dissolubility was seen in Chapter VI as the keystone of all experiment. The immense power attainable by work with artificial controlled systems becomes ours only as we bring that principle to bear to isolate and subdivide our problems. Quite recently we first learned that at the microcosmic level the "part" we can hope to isolate is not an event as such, but only the unit of event and apparatus required to render it as some observable large-scale effect. Aside from this minor qualification, however, quantum mechanics leaves untouched our habitual exploitation of dissolubility in the design of experiments.

In thought we proceed exactly as we do in experiment. Beginning with concepts of discrete objects and distinct phenomena, we resolve the world into "parts" we consider in abstraction from the whole. By way of superposition of these more comprehensible parts, we seek the whole that is often conceptually inaccessible in itself. But the superposability this program confidently assumes may well fail to 
materialize if the parts considered are insufficiently "natural." And du Noüy underlines this difficulty with his vigorous assertion that completely natural parts are completely unattainable, just as Aristotle contended ( see p. 152):

When we speak of a phenomenon, we speak only of an event, or of a succession of events, arbitrarily isolated from the universe whose evolution they share. By isolating a fact in order to study it, we give it a beginning and an end, which are artificial and relative. In relation to the evolution of the universe, birth is not a beginning, and death is not an end. There are no more isolated phenomena in nature than there are isolated notes in a melody.

Undeniably, our first efforts at theoretical comprehension ordinarily center not on the complex general case actually encountered, but on some highly restricted special case-an ideal "phenomenon" conspicuously artificial in its simplicity. To begin almost any theoretical problem may then demand something of that ruthlessness, recklessness, noted in one case by Pirie.

Nowadays most thoughtful biologists realize that the evolutionary origin of species is bound to give intermediate types and there may be a transient continuity between adjacent species. But anyone combatively aware of this in the eighteenth century was a nuisance. Then the most useful approach was a certainty that this is this and that that, and that the trouble with the small proportion of doubtful specimens was simply due to lack of knowledge.

So slashing away with conceptual scalpels, we can count as success only an operation in which we do recover our patient alive, entire. We know we have succeeded when, after thinking about parts, we can in thought reconstitute wholes. After abstracting from "nonessential complications," we must in the end render just account even of them-precisely as in the case just noted, where we do at last reintegrate the intermediate types, and so grasp both the distinctness and the continuity of "species."

Superposition, not summation. The whole we seek to comprehend may well differ from any sum of parts. Even common sense recognizes that a mob is no mere sum of constituent individuals: it has certain new "qualities," and lacks others found in individuals. Thus a correct superposition may sometimes demand-beyond addition of parts-due attention to the mutual interactions of those parts. En- 
counter with such a case is always something of a surprise-so general is our success in distinguishing substantially independent parts. To mark our surprise we usually give a special name to the "cooperative phenomenon" that redresses the balance of sum and whole. But, however it may surprise us, such a phenomenon does not dismay us: frequently it represents nothing more than some element(s) intrinsic to our problem, but purposively ignored in our first sketch of a solution.

Consider the "perturbations" of planetary orbits. The word may be seriously misleading: what we call perturbations are no more than artifacts of our own approach to the problem of calculating a planetary orbit. Ordinarily we begin with the simplifying assumption that planet and sun can be treated as completely independent of all other bodies in the system. This assumption yields a tractable twobody problem, but of course it is an oversimplification: there must also be gravitational attractions among planet, sun, and all other planets in the system. Directly to attack the total problem is hopeless: even a three-body problem defies exact mathematical analysis. We proceed then by solving the central two-body problem, and later re-introduce (as "perturbations") the generally minor systemic interactions previously ignored. And our success in thus reconstituting the whole from its parts is at least partially attested by the exquisitely accurate predictions we found on our celestial mechanics.

This instance has nothing unique about it. Thus we first approach the problems of nuclear physics with concepts of independent particles. However, among "particles" present in the narrow compass of the nucleus there might well be strong interactions. These do indeed manifest themselves in the difference between the mass of the nucleus and the sum of the masses of the particles of which we suppose it constituted. We call this difference a "mass defect," but we readily understand that the "defect" arises simply from the interaction of particles we formerly treated as wholly independent. Quite similarly, in chemistry we begin the study of molecular structure with a conception of bonds having characteristics dependent only on the identities of the two atoms directly linked by each bond. But ultimately we recognize co-operative effects of "parts" in the context of the molecule as a whole-systemic interactions for which we must make allowance, e.g., as "resonance."

How often we meet this situation in work with living organisms! 
We seek to understand the characteristics of lichens by viewing them as aggregates of algae and fungi, which parts we study in abstraction from each other. But of course the properties of a lichen are not simply the sum or average of alga and fungus properties. There is a co-operative phenomenon to which, as usual, we give a special name: symbiosis. Symbiosis is not something new and mysterious; it symbolizes just those interactions originally neglected. In pharmacy we come to consider (as "synergism") the special features of the joint action of two drugs originally considered singly; in medicine to consider (as "psychosomatic") phenomena that seem to involve the interaction of the two polarities-mind and body-physicians ordinarily treat in complete abstraction from one another.

A very few modern investigators have joined Driesch in maintaining that, by its approach through "parts," science condemns itself to eternal ignorance of wholes clearly more than any sum of parts. Whatever superficial plausibility this argument may present, I hold it fundamentally specious. Given "parts," often the able scientist can conceive wholes! What could be more outrageously arbitrary than the parts into which the embryologist's microtome cleaves his specimen? But from hundreds of these flat "sections" the skilled embryologist recreates in thought the three-dimensional totality of the intact embryo. Far more than that, by carrying through the same operation with a whole series of embryos of different age, he at last grasps the entire course of development of the single intact embryo. Behold the completed superposition of parts that could scarcely be more "unnatural"!

I find no basis whatever for the happy confidence with which obscurantists predict the defeat of science in the face of "the great problem of life." The life of an organism we seek to comprehend in terms of parts: anatomical, histological, and molecular. But no scientist is simple enough to suppose that the organism can in thought be reconstituted without due attention to the interactions of these parts on macroscopic, microscopic, and molecular levels. We hope ultimately to find "life" emergent from chemical systems the components of which are not alive in any meaningful sense; this alone could for us represent a rational explanation. Far from being an annoyance or disaster, the co-operative phenomena that make superposition different from summation are, in fact, the essential presupposition of every attempt to explain the qualities of wholes. 
Superposition and explanation. The emergence of new qualities demands context $(s)$ of interaction: certainly the interactions of one or more "entities" with an environment, and usually also the interactions of multiple entities (like or different in kind) with each other. A gas molecule has mass and motion, but no "springiness." A large number of such molecules in a particular context (e.g., a closed cylinder with movable piston) do manifest that behavior described by Boyle as "the spring of the air." And now, however clearly we may formerly have grasped the denotation of "gas pressure," so conceived emergent from molecular impact "gas pressure" acquires for the first time an explanation. Here, to a first approximation, the "outer" (environmental) context provides the only interaction required for emergence of a new "quality" from molecules present in bulk. Consider, however, another case. Water is "wet," we say, but there is no wetness to a water molecule. The emergence of wetness demands the presence of many water molecules in an "outer" context providing, at least, the conditions of temperature and pressure in which water is liquid, as well as any other circumstances required for the manifestation of wetness (we observe no wetness if all surfaces in contact with the water are waxed, for instance). But now we must consider also the "inner" context of interaction among water molecules present in bulk. Wetness indeed depends on the dominance of one interaction over the other, i.e., the attraction of water molecule and test-surface molecule outweighing the attraction of water molecule for others of its kind. Wetness thus at last emerges from a double context of electric interactions not meaningfully "wet" or "dry."

The qualities of the Sahara may be supposed "emergent" from the interactions, with the environment and with each other, of an immense multitude of grains of sand. The individual grain has no dunes -the quality of being duned develops from the presence of an outer context (e.g., winds) and an inner context (e.g., rolling friction between grains of sand). Dunes are so explained-and I do not venture beyond that to the much more ambitious contention of Sherlock Holmes:

From a drop of water a logician could infer the possibility of an Atlantic or a Niagara without having seen or heard of one or the other.

This Watson at least held "ineffable twaddle," and perhaps it is. Holmes' claim is one I neither affirm nor deny: I hold it completely 
irrelevant to the conception of emergence I have sketched. From such emergence I seek not the prediction of qualities or phenomena unlike any I have previously known to exist but, on the contrary, explanation of known qualities or phenomena for the existence of which I seek some "sufficient reason." This is, I think, just the sense in which corpuscular hypotheses may be said to explain qualitative change. They sketch for us mechanisms of change involving the collective deportment (e.g., relative displacements) in certain contexts of certain unchanging entities. So taught to understand how change may come about, we grasp a "sufficient reason" we may call the cause of change. And with that dread word we enter now a new area of discourse.

\section{The Principle of Determinism, and Causality}

Though treated here as a separate principle, determinism may perhaps be regarded as further corollary to the principle of continuity. The argument might run thus: Acting on the principle of dissolubility, we suppose the condition or state of a finite system adequately defined by specification of a small number of parameters. If a state A of some such system is forever succeeded by state $A$, we have the perfect continuity of identity. However, if instead state $\mathrm{A}$ is once succeeded by some other state B, we expect to find continuity maintained at least to the extent that, in other such systems, in other times and places, state A will always be succeeded by state B. The principle of determinism asserts that this element of continuity will be found if "proper" specifications of the states are given. We then attack the problem of establishing such specifications: each particular change we would show determined by a distinct antecedent state recognizable in advance of the event.

When change occurs state $\mathrm{B}$ is the effect and, in whole or in some part, state $\mathrm{A}$ is the cause. But the idea of causality transcends any such trivial matter of naming and, however closely associated with the idea of determinism, it transcends that as well. Establishment of continuity in determinism satisfies the Egyptian quest for order that change may be rendered predictable. But the causal concept is rooted in the deeper and more difficult Greek quest, for understanding, broached in the principle of intelligibility. That is, if determinism expresses the conformity of phenomena to laws, in a universe 
ruled by "necessity," then causality expresses the aspiration to grasp the nature of that necessity as distinct from the form of those laws. Some modern physicists, like Weizsäcker, seek to reduce causality to nothing more than determinism.

The criterion for the fact that one really knows the efficient cause, is that one can predict correctly the event produced by it. Thus the concept of cause has been so transformed, that the causal principle in modern natural science has been identified precisely with the complete predictability of natural phenomena.

But though understanding may be manifested in the capacity to meet this "criterion," it is not realized in, or constituted by, that capacity. Through causality we seek, beyond knowledge of what-whenwhere, a grasp of the how of phenomena.

The aspiration to grasp how was, we saw earlier, entirely responsible for the naturalistic construction the Greeks put upon the principle of determinism. And observe now in another instance at the very beginning of the modern era that, once again, it is clearly the conception of causality which controls application of the principle of determinism. Thus Galileo laughs to scorn an alleged determinist order simply on the ground that the causal connection it implies is absurd:

If Sarsi [Grassi] wants me to believe with Suidas that the Babylonians cooked their eggs by whirling them in slings, I shall do so; but I must say that the cause of this effect was very different from what he suggests. To discover the true cause I reason as follows: "If we do not achieve an effect which others formerly achieved, then it must be that in our operations we lack something that produced their success. And if there is just one single thing we lack, then that alone can be the true cause. Now we do not lack eggs, nor slings, nor sturdy fellows to whirl them; yet our eggs do not cook, but merely cool down faster if they happen to be hot. And since nothing is lacking to us except being Babylonians, then being Babylonians is the cause of the hardening of eggs, and not friction of the air."

Today, as noted at the end of Chapter VI, strictly analogous causal considerations dominate our appraisals of statistical correlations of data. Because we readily conceive a causal mechanism, we are predisposed to judge significant a statistical correlation between cigaret smoking and the incidence of lung cancer; and equally predisposed 
to judge non-significant statistical data alleged to indicate an extrasensory perception (ESP) for which we can imagine no causal explanation.

The view of causality here proposed is, of course, kin to Leibniz' principle of sufficient reason. However, this principle can assume two quite different variants. Leibniz himself adopted the cosmologic variant that asserts something about nature, e.g., for each observed effect there exists an assignable physical cause or causes. Far different is the methodologic variant that, to guide $u s$, asserts a heuristic maxim, e.g., for each observed effect seek to assign a physical cause or causes. A covert cosmologic implication attaches, we saw, to any recommendation of policy for dealing with nature; but in this hortatory sense "sufficient reason" becomes the primarily methodologic principle I equate with the conception of causality.

Causality as heuristic maxim. Planck considered causality

. . . a heuristic principle, a signpost-and in my opinion, our most valuable signpost-which helps us find our bearings in a bewildering maze of occurrences, and indicates the direction in which scientific research must advance in order to arrive at fruitful results.

The "direction" is simply that in which, setting out from an observed effect, we pursue the search for a causal mechanism competent to produce that effect. Hume's long-standing demonstration that causes cannot be assigned with certainty is, fortunately, much easier to ignore than to refute. And, however far "true causes" may (or may not) lie beyond our grasp, the search for such causes has proved immensely rewarding. One might then say of the idea of causality what Bacon said of alchemy:

Alchemy may be compared to the man who told his sons that he had left them gold buried somewhere in his vineyard; where they by digging found no gold but, by turning up the mold about the roots of the vines, procured a plentiful vintage.

Just so, we discover the conditioned reflex while seeking the cause(s) of behavior.

The word "cause" has a highly variable status in the scientist's vocabulary. Talking about his work in progress, he is apt to voice, and act on, various speculations about the causes of certain observable effects. But when his work is completed the word "cause" may 
not even appear in his statement of findings. Imagine, for example, that I am a pre-Copernican astronomer puzzled by the striking fact that when an exterior planet retrogrades it always stands in opposition to the sun. Finding this correlation inexplicable within the framework of the Ptolemaic system, I set myself to discover its origin, and perhaps ultimately I hit upon the Copernican theory. I then see how the odd correlation of retrograde and opposition may be caused and, in my exposition of the Copernican system, I may well cite this convincing explanation as an argument for acceptance of that theory. But, quite emphatically, I will not state that the Copernican system is the cause of this notable correlation. In somewhat the same fashion, first tidings of the existence of the Andes mountains may at last explain for me a previously known occurrence of a large high-altitude city like Quito, but I am unlikely to say that the Andes cause Quito's high altitude. The scientist's search for causes is but means to the end of understanding and, that understanding once achieved, his grasp of an entire system of phenomena and explanations can no longer be expressed in necessarily fragmentary statements about particular causes of particular phenomena.

This curious disappearance of "cause" from the ultimate statements of knowledge achieved through pursuit of causes encourages misinterpretation of the historical record-misinterpretation all the more likely because several of the earlier scientists seem expressly to disclaim any interest in causes. Thus Galileo puts into the mouth of Salviati the statement that:

The present does not seem to be the proper time to investigate the cause of the acceleration of natural motion, concerning which various opinions have been expressed. . . . At present it is the purpose of our Author merely to investigate and to demonstrate some of the properties of accelerated motion (whatever the cause of this acceleration may be). . .

In entirely similar fashion Boyle mentions the two models (plenist and kinetic) that might explain the origin of the law that bears his name, but he declines "to declare peremptorily for either of them against the other" on the ground that:

I shall decline meddling with a subject, which is much more hard to be explicated than necessary to be so by him, whose business it is not, in this letter, to assign the adequate cause of the spring of the air, but 
only to manifest, that the air hath a spring, and to relate some of its effects.

In their time these passages expressed a novel and important idea: the value of even fragmentary knowledge, i.e., isolated laws. Both authors also imply that a search for more complete understanding then seemed unpropitious, but neither implies that such understanding is unattainable. As a matter of fact, Newton did of course find causes for the acceleration of falling bodies and the springiness of air. We construe the second case in a very different manner, but the "adequate cause" is for us, as for Newton, an explanation in terms of forces.

What of the celebrated Newtonian disclaimer: hypotheses non fingo? What Newton actually says is this:

But hitherto I have not been able to discover the cause of those properties of gravity from phenomena, and I feign no hypotheses; . . . to us it is enough that gravity does really exist, and act according to the laws which we have explained, and abundantly serves to account for all the motions of the celestial bodies, and of our sea.

The word "hitherto" is illuminating. Like Galileo and Boyle, Newton wisely declines speculation when, for the purpose in hand, he felt the need as clearly insufficient as the evidence. But this is a renunciation neither final nor complete. Far from it! In the very next (concluding) paragraph of the scholium in which the above passage appears, Newton considers certain possible causes of gravity. He remained passionately interested in the problem, and never despaired of solving it. Certain speculations offered in the later editions of the Opticks left him still unsatisfied, but his feelings remain apparent in the words I have italicized in the following famous passage.

To tell us that every species of things is endowed with an occult specific quality by which it acts and produces manifest effects, is to tell us nothing: but to derive two or three general principles of motion from phenomena, and afterwards to tell us how the properties and actions of all corporeal things follow from those manifest principles, would be a very great step in philosophy, though the causes of those principles were not yet discovered; and therefore I scruple not to propose the principles of motion above mentioned, they being of very general extent, and leave their causes to be found out. 
If legend may be trusted, Newton's entire gravitational theory originated in the speculation that the fall of the apple and the "fall" of the moon in its orbit have one and the same cause. Let no man presume to declare the search for causes unessential to Newton's work. Leaping all the way to Planck, at the beginning of the present century, we find still active in him a concern for causality wholly unsatisfied in the establishment of determinism. Studying blackbody radiation, Planck at last discovered a mathematical expression for the variation of intensity with wavelength and temperature. That law reduces blackbody phenomena to determinist order; but Planck sought, beyond this, a causal explanation of how there is produced the behavior his law describes. Pushing on in this endeavor, he arrived at the oscillator model that first suggested the occurrence of a quantum of action. And here we meet the crowning irony: the conception so first born matures into a quantum mechanics that challenges not only the idea of causality that inspired Planck's work, but even the concept of determinism.

\section{QUANTUM MECHANICS, DETERMINISM, AND CAUSALITY}

By the testimony of professional metaphysicians, and of scientists who speak as metaphysicians, we are assured that a catastrophe in the domain of microphysics now forces on us a complete revision of our concept of causality and determinism. Yet we behold with astonishment that scientists still persevere in causal inquiries that, as ever before, terminate fruitfully in conclusions that make no reference to causes. This paradox is at once resolved when we recognize the cosmologic locus of the "catastrophe." The heuristic principle of causality is far less affected than a cosmologic conception of determinism that, starting perhaps from the God who sees the sparrow's fall, passes on to a Laplacean non-God who precisely foretells the coming of star, dust-mote, and electron. Such an absolute determinism of individual events had of course never been attainable in the practice of science, and scientific practice owed but little to it. Science thus remains undamaged when the cosmologic conception of determinism is toppled by the explosive charge that individual microphysical events remain forever subject to an irreducible indeterminacy and unpredictability. But that blast does have some important repercussions in science. 
Mendel's laws do not permit us to predict the characteristics of the plant that will grow from a given seed of known hybrid pedigree. This check does not much stir us: lacking knowledge of the exact "state" of the seed concerned, of course we cannot predict. And in microphysics, too, it was argued by Planck and others that a precisely analogous incapacity for prediction derives from a precisely analogous deficiency in practice of knowledge conceivably attainable in principle. Today most physicists reject this view, contending that microphysical indeterminacy is no matter of what we don't know but, rather, what we can't give meaning to even in principle. Precise knowledge of the initial state of a microphysical system is impossible for the simple reason that the defining parameters of state are irreducibly statistical in nature. Thus statistical variation of results, leaving the individual event substantially uncertain, arises not from our ignorance of the initial state of the system, but from that state as such.

Let us concede this widely accepted opinion. What then? This conception leaves entirely unshaken the dominance by determinism of the kind of large-scale events already known reducible to determinist order. Moreover, as Margenau observes, we can still say something meaningful about the initial states even of microphysical systems.

. . . if the condition of the particle is unspecifiable by statements saying where it is and how fast it is going; if it is found sometimes here and sometimes there, then an aggregate of measurements must be performed, and the interesting information is the relative frequency, i.e., the probability, with which it is found here or there. It is difficult to see why an observable should lack the fitness to serve as a variable of state if its determination requires more than one measurement.

Even at the microphysical level the initial state of a system can thus be defined, in statistical terms, and so of course we can make predictions for statistically large numbers of events. I have now no basis for predicting when some one particular radioactive atom will decay. But I easily predict, and with great exactness, the time required for decay of some given fraction of a very large number of such atoms.

Even in microphysics, then, determinism is a far from total loss. But what of causality: can we still aspire to know the how of microphysical phenomena involving parameters only statistically signifi- 
cant? I believe we can, and do. As science matures, so does our conception of causality. At the inception of modern science action-bycontact was the only causal mechanism fully acceptable; and the "unthinkable" notion that celestial bodies can attract each other from a distance, through "empty space," was as repugnant to Newton as to most of his adversaries. But, as earlier suggested, ultimately we learn to find acceptable explanations in models other than those that involve directly only mechanical pushes and pulls. Today, in confrontation of the phenomena of microphysical indeterminacy, the concept of causality undergoes not destruction but a still further enlargement.

For an observed microphysical effect we still seek, if not causes, then reasons, e.g., involving "chance" and the law of large numbers. May it be objected that, of its very nature, "chance" cannot furnish a sufficient reason? Suppose I find that in a large number of throws of a well-balanced die each face has turned up very nearly one-sixth of the time. For this observation have I not an amply sufficient reason when I recognize the absence of any cause that favors one face as against another? I feel in this sufficient explanation of how to requite, if not wholly to satisfy, the quest for sufficient reason broached by the concept of causality. For to me it appears, as to Landé, that:

. . random events take place continually, as C. S. Peirce remarked long before the quantum age. There are not sufficient causes for every event.

On the other hand, there is sufficient reason for the lack of sufficient causes when one accepts Leibniz's principle of cause-effect continuity. This principle leads first to the conclusion that the reaction "always yes" or "always no" respectively should be bridged by intermediate reactions "sometimes yes and sometimes no," that is, by cases of indeterminacy in an individual test, dominated by statistical averages.

The destruction of the cosmological principle of determinism does not, of course, leave even the purely heuristic concept of causality entirely unscathed. Thus Planck observes that:

If it is assumed that statistical laws are the ultimate and most profound in existence, then there is no reason in theory why, when dealing with any particular statistical law, we should ask what are the causes of the variations in the phenomena? Actually, however, the most important advances in the study of atomic processes are due to 
the attempt to look for a strictly causal and dynamic law behind every statistical law.

If science is genuinely transformed in microphysics, Planck's historical precedents will not compel us to join him in resisting the alleged ultimacy of statistical laws. But a recent statement of de Broglie's suggests that, even yet, there may be some substance to Planck's misgivings.

For a quarter of a century the purely probabilistic interpretation of wave mechanics has certainly been of service to physicists because it has kept them from being overwhelmed by the study of very arduous problems ... and thus has permitted them to advance steadily in the direction of applications, which have been numerous and fruitful. But today the heuristic power of wave mechanics, as it is taught, seems in large measure weakened. Everyone recognizes this . . .

However this may be (for "everyone" does not "recognize this"), the widely alleged ultimacy of the statistical view lies open to attack on what seems to me a far more fundamental plane. The target then is not the statistical view as such, but the more vulnerable pretension to knowledge of a final principle.

Quantum mechanics produces appreciable changes in our conceptions of determinism and causality. But precisely because these are not the first such changes, we may well doubt they are the last. In the general context of his beliefs, the man of the Dark Ages could demonstrate convincingly the absolute unpredictability of the appearance of comets-divinely sent portents of disaster. In much the same way, within the context of early 19th-century science, one could produce an apparently conclusive demonstration that the construction of heavier-than-air flying machines was impossible. Yet behold, we are borne upon the air and we foretell the coming of comets. Presuming the finality of the context of modern quantum mechanics, one can prove the ultimacy of statistical laws, the irreducibility of the wave-particle dualism, and I know not what else. But Bohm correctly emphasizes that the crucial initial presumption is neither demonstrated nor demonstrable.

. . conclusions have been drawn concerning the need to renounce causality, continuity, and the objective reality of individual microobjects, which follow neither from the experimental facts underlying the quantum mechanics nor from the mathematical equations in terms 
of which the theory is expressed. Rather, they follow from the assumption (usually implicit rather than explicit) that certain features associated with the current formulation of the theory are absolute and final, in the sense that they will never be contradicted in future theories ... .

Such assumptions have in the past proved groundless often enough to encourage a watchful reservation as regards, for example, the "principle of complementarity" that Landé equates with:

. . . the doctrine that a fundamental wave-particle duality is an immanent feature of the microcosm which must be accepted at face value without permitting any further explanation. To my mind, this doctrine relies too much on the policy: if you cannot explain it, call it a principle; then defend it as fundamental and absolutely irreducible, so that speaking of the unsolved "riddle of duality" from here on becomes the mark of naïveté if not of heresy.

I do not presume to know the outcome of the highly technical dispute still in progress. But I feel quite sure that always scientists must resist the finality of assertions about human incapacity for knowledge. Thus, for example, if I accept as final the view of vitalism, I will inevitably make it "true" by foreswearing just that effort, to comprehend living organisms, which is alone competent to demonstrate the falsity of vitalism. But here I trespass on a much larger matter.

\section{The Principle of Corrigible Fallibility}

We have earlier touched upon a point made with great emphasis by Nietzsche:

The assertion that the truth is here, and that an end has been made of ignorance and error, is one of the greatest seductions that there are. Assuming that one believes it, then the will to test, investigate, predict, experiment, is crippled: the latter can itself become wanton, can doubt the truth. The "truth" is consequently more ominous than error and ignorance because it binds the forces with which one can work for enlightenment and knowledge.

To resist "the truth" is difficult. The more deeply an open question concerns us, the more we crave its final resolution. Of all human enterprises, only science has succeeded in fathering some approximation to that attitude of philosophic doubt of which Cohen says: 
As the state of doubt is intensely disagreeable, communities try to get rid of it in diverse ways, through ridicule, forcible suppression, and the like. The method of science seeks to conquer doubt by cultivating it and encouraging it to grow until it finds its natural limits and can go no further. Sober reflection soon shows that though very few propositions are in themselves absolutely unquestionable, the possibility of systematic truth cannot be impugned.

The last sentence of Cohen's statement is of pivotal importance. A rampant Pyrrhonism was no small factor in the decline of ancient science. Unalloyed doubt, eventuating in nothing but a paralyzing awareness of the possibility of error, is as fatal as the complete assurance remarked by Nietzsche. Implicit in all action is necessarily the possibility of wrong action: we can be certain we have done nothing wrong only by taking care to do nothing at all. Science can progress in its pursuit of truth only as it passes over from timid avoidance to bold acceptance of the risk-nay, the certainty-of error.

\section{LEARNING PRESUPPOSES ERROR}

We find in Genesis that learning presupposes failure, error; and Carr's maze-running tests show that, for men as for mice, learning occurs only when the subjects are allowed to make and recognize their errors. Learning thus presupposes error and, as itself a process of learning, science presupposes error. Not simply accommodating ourselves to the possibility of error, we actively court error (e.g., by the extreme generalization of our laws and theories). Only through error, recognized as such, can we hope to learn. "The capacity of learning from experience," says William James, "is one of the rarest gifts of genius." The learning act that both demands and manifests such genius may be precisely that first difficult detection of error in an erstwhile indubitable. The policy of science is to maximize the possibility of learning by minimizing the number of "truths" held permanently beyond any challenge posed by experience. In energetic pursuit of the infallibility we confidently suppose humanly approachable, we can advance only to the extent that we deny ourselves the illusion that any item of infallibility is already attained.

Popper holds it a point of fundamental importance-to the philosophy of science no less than to the methodology of science-that the distinguishing characteristic of an empirical statement is its susceptibility to falsification. Stipulating that all scientific beliefs must be 
potentially falsifiable, we arrive at one belief we can hold comparatively certain simply because it has survived submission to the many tests that have discredited all known alternative beliefs. However "negative" this approach to scientific truth, it-and it alone-can justify the positive affirmation of Mill's ringing declaration:

The beliefs which we have most warrant for, have no safeguard to rest on, but a standing invitation to the whole world to prove them unfounded. If the challenge is not accepted, or is accepted and the attempt fails, we are far enough from certainty still; but we have done the best that the existing state of human reason admits of; . . . and in the meantime we may rely on having attained such approach to truth, as is possible in our own day. This is the amount of certainty attainable by a fallible being, and this the sole way of attaining it.

We here approach a characterization of science in negative terms. It is a method of rejection that invests science with the extraordinary power for self-correction Cohen holds to be decisive.

The apodictic certainty of science is not the absolute certainty of any specific result or material proposition, but the dialectic demonstration that any inaccuracy or false step can be corrected only by relying on principles inherent in the system of science itself. This is a position unassailable by any argument that can pretend to have any evidence in its favor.

Plainly a purely negative system cannot suffice. Any expectation of progress through a method of rejection must always presuppose a positive production of new items thus to be winnowed. However, the human mind seems quite competent to produce new scientific ideas, and its competence most seriously impaired by unavailability or unacceptability of a reliable criterion of rejection. We are most stimulated to produce new ideas by recognition of the inadequacy of ideas we have earlier accepted. Thus for science the negative capacity to court, recognize, and reject error is in itself precious. The characteristically strong development of that faculty in science is indeed the wellspring nourishing the root of scientific progressivism.

No longer do we expect science to reach any bedrock of absolutely secure knowledge. Today's conception of science, as endless frontier, was grasped by Bernard a century ago.

When we propound a general theory in our sciences, we are sure only that, literally speaking, all such theories are false. They are only par- 
tial and provisional truths which are necessary to us, as steps on which we rest, so as to go on with investigation; . . .

Given faith in the possibility of progress toward superior knowledge, we can afford to indulge that scepticism of present knowledge which holds even our best established theories potentially open to review. This is the sense of Thomson's dictum that a theory is "a policy rather than a creed," and the substance of a delightfully matter-of-fact statement by Lewis.

The scientist is a practical man and his are practical [i.e., practically attainable] aims. He does not seek the ultimate but the proximate. He does not speak of the last analysis but rather of the next approximation. . . . On the whole, he is satisfied with his work, for while science may never be wholly right it certainly is never wholly wrong; and it seems to be improving from decade to decade.

\section{THE MUTUAL CONTROL OF FACTS AND THEORIES}

Science is basically empirical, and all scientists share Newton's opinion that:

We are certainly not to relinquish the evidence of experiments for the sake of dreams and vain fictions of our own devising; . . .

But in the face of what evidence are "true propositions" to be relinquished as "dreams and vain fictions"? Generally the evidence must be overwhelming. For always it is we who must interpret "the evidence of experiments," and always we do so in the uncertain light shed by those "older truths" of which James says:

Their influence is absolutely controlling. Loyalty to them is the first principle-in most cases it is the only principle; for by far the most usual way of handling phenomena so novel that they would make for a serjous rearrangement of our preconception is to ignore them altogether, or to abuse those who bear witness for them.

Just so Galileo was abused for his telescopic observations. Just so we ignore the repeated appearances of microorganisms in media earlier supposed to have been sterile, and thus maintain the nonoccurrence of spontaneous generation. For us these appearances are not the "evidence of experiments" but only the evidence of experimental errors. However temptingly simple may be the identification of science with the policy of systematic doubt, we must recognize 
that science embodies always essential elements of systematic belief. An obdurate defender of his own opinions, Newton is fully aware that no theoretical ideas are susceptible to any perfectly final demonstration.

And although the arguing from experiments and observations by induction be no demonstration of general conclusions; yet it is the best way of arguing which the nature of things admits of, and may be looked upon as so much the stronger, by how much the induction is more general.

Yet Newton also recognizes that, however far beyond possibility of demonstration our best established ideas may lie, it is essential that we cling to them. He indicates the importance he attaches to this view by framing his fourth Rule of Reasoning to run as follows:

In experimental philosophy we are to look upon propositions inferred by general induction from phenomena as accurately or very nearly true, notwithstanding any contrary hypotheses that may be imagined, till such time as other phenomena occur, by which they may either be made more accurate, or liable to exception.

The double thrust of this rule is decisive. From it we gain courage to rely on unprovable theoretical ideas in the design and interpretation of our experiments. In the face of apparent contradictions we defend our theories stoutly, and accept their guidance as we press on to new investigations. But precisely these endeavors, together with Newton's conception that all theoretical ideas are potentially liable to exceptions, may bring us ultimately to a very sceptical review of the ideas that have directed our efforts.

We arrive here at the very core of the principle of corrigible fallibility. The sense of fallibility encourages detachment, and the capacity to recognize error; the sense of corrigibility allows us to proceed hopefully, in full commitment to the ideas we believe well established. Commitment and detachment, but on somewhat different time scales. In the long term science expresses detachment: nothing is held permanently above all question. In the short term science expresses commitment, and this short-term commitment is every bit as important as the long-term detachment. Viewing with detachment is nothing if not backed by acting with commitment. Or, in James' vigorous statement: 
. . . we have to live today by what truth we can get today, and be ready tomorrow to call it falsehood.

Not quite tomorrow: to certain ideas we long maintain allegiance even in the face of glaring contradictions. And this is something we must do-for at least two reasons: From our steadfastness in the face of adversity, science first gains the continuity of an orderly evolution. And from our uniform adherence to one body of "established" doctrine, science first gains the character of a corporate enterprise.

\section{Substantive Principles}

In what does a mountain-climbing expedition differ from a haphazard scramble of individuals in a wilderness? The expedition can function as such only if its members think and act alike in certain basic matters. All agree to climb that mountain, using these techniques, and departing from this base camp. At progressively higher altitudes advanced camps will be established at sites on which, again, all must agree. Suppose the climbing party finally confronted by some obstacle that defies every effort made with the climbing techniques they are equipped to practice. They must then cast around for some alternate route, to exploit which one or more of the higher camps may have to be shifted-perhaps to locations earlier urged unsuccessfully by some members of the party. A route that demands relocation of only one, or at most two, of the higher camps will perhaps be worth considering. But the party's resistance to each suggested alternate route increases rapidly with the number of such relocations its use demands. If all the advanced camps and the base camp must be moved to the other side of the mountain, the expedition acknowledges its defeat.

Mounting their assault on some particular experiential mountain, the scientists of a given generation bring to the ascent various conceptual and experimental techniques, applied under the direction of the regulative principles all accept as definitive. They take their departure from a base camp of substantive principles-laws and theories all accept as essentially indubitable. Prospecting the ascent, conceptual camps (i.e., less firmly held hypotheses and laws) will be established at progressively greater altitudes. Major disagreement on the location of these does not break out until the climbing party finds itself in serious difficulties. If attempts to overcome these difficulties 
fail, a new route to the top is sought. A new route requiring relocation of one or two of the high conceptual camps allows the enterprise to go on with little loss of momentum; but resistance to such relocations rises swiftly with the number of them required. The situation of the lower conceptual camps will be called in question only after longcontinued failure. The situation of the base camp of substantive principles will be challenged only when every other alternative has failed.

Our ideas arrange themselves in a hierarchy. At the foundation level occur those laws and theories we hold as of principle, while at the growing edge of science appear highly tentative hypotheses we are quite prepared to re-examine. These are the extremes of a pecking-order that stretches all the way from certainty to doubt. This conceptual hierarchy, readily understandable on historical and psychological grounds, has also a logical basis to which Toulmin directs attention.

One cannot at the same time question the adequacy of Snell's law and go on talking about refractive index. . . . Certainly every statement in a science should conceivably be capable of being called in question, and of being shown empirically to be unjustified; for only so can the science be saved from dogmatism. But it is equally important that in any particular investigation, many of these propositions should not actually be called in question, for by questioning some we deprive others of their very meaning. It is in this sense that the propositions of an exact science form a hierarchy, and are built one upon another; ... .

The terms "established" and "hypothetical," as used in science, need to be understood in terms of the distinction between the parts of science that are actually being called in question, and those which we must take for granted in order to state our working problems.

The "established" laws and theories we take for granted define a background of presupposition. As earlier noted, we need this even to recognize (as divergences from what we expect) the "phenomena" for which we will seek explanations. Thus, given the standard of normality set by Snell's law, double refraction is unmistakably a phenomenon-producing what we call, significantly enough, an "ordinary" ray and an "extraordinary" ray. For the mountain-climbing expedition the location of the base camp affects decisively the chance of ultimate success, by predetermining the general area in which higher camps can be established. For science the standard of the 
"natural" established by one or a very few items in the foundation level of substantive principle may predetermine the general direction, and the success or failure, of the scientific endeavor of entire generations. For example, consider how for millennia men wrestled unsuccessfully with the problem of motion. That problem is completely restructured with the coming of a new substantive principle: the Newtonian concept of inertia. Continued uniform rectilinear motion, something never observed in nature, is now considered "natural" and in need of no explanation. Only changes of motion (some of which had earlier seemed entirely "natural") now qualify as effects for which causes will be sought. One then no longer asks how it is that a projectile is maintained in motion-a query that proved unanswerable. Instead one asks how it happens that a projectile moves not in a straight line but along a curved path-a query that proves satisfyingly answerable.

What are for us the ultimate substantive principles? I do not know. Much must depend on the problem at issue. For terrestrial phenomena on the molar scale presumably Euclidean geometry, Newtonian mechanics, and the various conservation laws will represent substantive principles as ultimate as any. Are the conservation laws really substantive principles? Consider that, as earlier noted, we simply multiply species of energy as required to find continuity in change. The strength of that determination then itself lends almost magic power to the concept "energy," of which Poincaré remarks:

. . . the word "energy" . . . made the law by eliminating the exceptions, since it gave the same name to things differing in matter and like in form.

Does the final conservation law then represent anything more than an expression of the determination with which we set out? Consider too that, though today the conservation of mass (or mass-energy) may seem no more than an exhaustively confirmed colligative relation asserted as a principle, it was pronounced as a principle by Lavoisier (among others) well in advance of that confirmation.

One can lay down as a principle that in every operation there is an equal quantity of matter before and after the operation.

Are all conservation laws simply expressions of our determination to find continuity, and thus simply human conventions? Are all substantive principles simply conventions? 
Substantive principles as conventions. Substantive principles are conventions in that they represent an unforced choice of a particular point of departure agreeable to all scientists. They are conventions, further, in that they constitute the ultimate reference standards against which "phenomena" are measured. Still further, in that ordinarily we maintain them come what may. But to dismiss them as wholly conventional is to fail entirely to render due account of their experiential provenance and control. Some such principles were once held very tentatively, and only after confidence in them seemed justified were they removed from the arena of doubt to the sanctuary of comparative certainty. Others entered the sanctuary wholly unnoticed-too "obvious" ever to have been called in question. Once in the sanctuary none of these principles is ever challenged until we find ourselves in deep and prolonged difficulties. But given such difficulties, any of them becomes subject to recall to the "proof" of the arena of doubt.

For more than a thousand years after Ptolemy all astronomers accepted at least two assumptions as indubitable principles. First, that the earth is at rest at the center of the universe; second, that celestial bodies move in paths somehow compounded from circles-"perfect circular motion," says Hall, "was an unquestioned cosmological principle." The first constitutes the earth the origin of stationary reference axes against which celestial motion is measured; the second defines the form to be sought in all such motion. With Copernicus the first principle is revised (the sun becomes the reference center) but the second is left untouched. With Kepler the sun remains the reference "center" (though only as one of two foci) but the second postulate is revised to permit motion in perfect ellipses. With Newton the fixed reference point recedes (perhaps to the "fixed" stars) and the second postulate is wholly rejected: due to perturbations, the motion of no planet conforms perfectly to any ideal geometric figure. With Einstein, finally, the first postulate is also rejected: there are no privileged reference axes. Thus the two principles, at one time firmly conventionalized, first change and finally vanish. A third "conventional" principle has, however, remained in force throughout: always some of the spots of light in the night sky have been taken as signs of "existing objects."

At first sight all conservation laws may seem no more than various purely conventional expressions of the principle of continuity we 
are determined to maintain. But of course all conservation laws do not prove equally adequate. We fail to find conservation of volume, or of motion as such, and even the conservation of mass and energy is not now construed as it was a century ago. Even that foundation of all conservation laws, the principle of continuity, is not left unaltered by the discovery of quantum phenomena.

Few, if any, substantive principles are "mere conventions." Whether we suppose the earth moving or at rest is perhaps in part a matter of convention for, as Galileo recognized,

. . all experiments that can be made upon the Earth are insufficient means to conclude for its mobility but are indifferently applicable to the Earth movable or immovable; . . .

But long before the age of interplanetary rockets we had learned to reject our earlier preference for a stationary earth; and Painlevé can, I think, insist with reason that:

If it is a convention to say that the earth rotates, it is equally a convention to say that it exists, and these two conventions are justified by identical reasons.

We distinguish between "primary" and "secondary" qualities, and seek to explain the second in terms of the first. For example, imputing only the first to hypothetical microcosmic particles, we hope to find "emergent" the secondary qualities of ordinary bodies. Newton thought the distinction of primary and secondary qualities of sufficient importance to touch on it in his third Rule of Reasoning:

The qualities of bodies which admit of neither intensification nor remission of degrees, and which are found to belong to all bodies within the reach of our experiments, are to be esteemed the universal [and primary] qualities of all bodies whatsoever.

Berkeley showed that this distinction, and others like it, fail of adequacy, and Ayer comments that:

The famous distinction which Locke drew between primary and secondary qualities is not a distinction between those perceived qualities that are unaffected by the conditions of observation and those that are affected. Since all are affected, there is no such distinction, as Berkeley realized. The primary qualities of the object, those that literally characterize it, are, on this view, just those properties with which science credits it. 
Thus dependent on the accreditation of human scientists humanly fallible, is the distinction of primary and secondary qualities anything more than a convention?

Observe the change of classification with time. For Aristotle hotness, coldness, wetness, and dryness were the primary qualities, and this we deny. For Newton hardness was a primary quality, which we deny, and the "charge" we regard as primary could not so be regarded by Newton. At any one time the adoption of a single classification is essential: only so can the scientific endeavors of an age gain coherence and mutual relevance. Our present classification seems well justified by notable successes won with it in the past. But if future efforts fail to yield equally notable finds then, if only after prolonged struggle, we will again learn a new and better classification.

The distinction of primary and secondary qualities focuses attention on certain types of explanatory endeavors earlier found fruitful, and correspondingly diverts attention from others earlier found unrewarding. In this last function the distinction is affiliated with a large group of inhibitory principles, all having the form: "Thou shalt not waste thy powers in vain endeavors." What endeavors are considered "vain" will vary with the development of science. Late in the 18th century the French Academy announced that it would no. longer consider papers on perpetual-motion machines. Essentially this made official a judgment that attempts to devise such machines are vain endeavors, and we have not since found occasion to revise this judgment. On the other hand, the transmutation of the elements that most responsible 19th-century scientists regarded as wholly vain -and quite rightly so, considering the means at their disposal-has been achieved in the 20th century.

An inhibitory principle is a warning posted at the edge of an abyss presently unfathomable. Some such principles (e.g., that rejecting the possibility of perpetual-motion machines) are justified by a long antecedent history of failure; others are justified on the warranty of apparently indubitable theories. By curbing endeavors all too probably vain in the contemporary state of science, they conserve scientific effort. Such a principle is of value regardless of its ultimate fate and, quite plainly, it is no "mere convention." 


\section{CHAPTER VIII}

\section{Theories and Models}

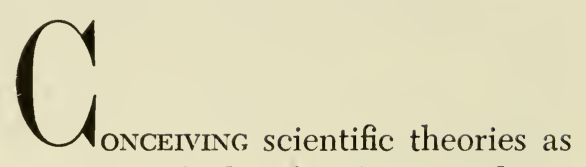

postulational systems, we begin again with that familiar paradigm, Euclidean geometry. Viewed as it is by mathematicians, of what does Euclidean geometry consist? We find (1) certain posits: "point," "line," etc.; and (2) a set of stipulated axioms. Following these come specifications (very incompletely indicated by Euclid himself) of (3) certain syntactic rules, the formal manipulations we are authorized to use in drawing deductions from the axioms. We then readily produce (4) the manifold of theorems of Euclidean geometry.

Euclidean geometry so conceived is a purely formal system. The axioms, expressing relations among the posits that figure in them, supply some implicit definitions of "point," "line," and so forth. But nowhere are denotations associated with these terms. All the other entities of Euclidean geometry "constructed" from the primary posits are then left equally devoid of experiential relevance. Through this novel conception of a wholly inapplicable abstract geometry (fully achieved only in the 19th century) we come to grasp the possibility of non-Euclidean geometries turning on somewhat different sets of axioms. These geometries are less familiar than Euclid's but-if, like his, each is a consistent development of a set of noncontradictory axioms-they are equally valid, because equally insusceptible to confirmation or falsification in the world of experience with which they have no link whatever.

Now all this seems absurd: we "know" that Euclidean geometry applies to the spatial relations of our terrestrial world. Quite so, but 
as non-mathematicians we conceive Euclidean geometry as something more than a formal system. Very much as Euclid himself presumably did, we conceive his system as highly endowed with experiential relevance-as quite literally a "geometry" usefully applicable to the measurement of land. And Euclid's formulation of his geometry does, indeed, embody one element so far unmentioned. He furnishes a set of explicit definitions of his posits. Formally these definitions are wholly superfluous: the Euclidean theorems stem not at all from them but only from the axioms. Once stated, these definitions make no subsequent appearance in the system, and they may well seem quite meaningless. For example, Euclid defines a point as "that which has no parts"; and a straight line as "that in which all points lie evenly on one another." But how are we to understand "point" if we are not told what is meant by "parts"? And an attempt to define parts involves us in either a potentially infinite regress or a circularity of greater or less extent. Not knowing the meaning of "lie evenly on," we are in no better position to recognize a straight line than a point. Purely verbal reformulation is of no assistance. Were we, for example, to define a straight line as the shortest distance between two points, then-waiving the difficulty of defining "points"-we would have still to grasp the meaning of "shortest distance." The attempt to define this in terms of the straight line is obviously an immediate and hopeless circularity.

Apparently nonoperative and entirely inoperable, Euclid's definitions are yet of major importance. For some two millennia, until mathematics had reached a very high level of sophistication, the inadequacy of Euclid's statement of syntactic rules was automatically made good by the intuition of geometers who never failed to assume what Euclid had himself taken for granted. And in much the same way, for much the same period, men have affiliated with his posits experiential denotations his definitions perhaps imply or evoke but certainly do not state. We may then, for example, take a "point" to be the point of a needle, or the fine hole left thereby in a thin sheet of metal, a "straight line" to be the path of a ray of sunlight selected by passage through that hole, and so on. Proceeding in this fashion, we identify certain of the hitherto wholly abstract terms of the formalism with concepts that have for us reasonably clear denotations, established perhaps around such tools as the ruler and compass with which we now proceed to "construct" the other entities of Euclidean 
geometry. Precisely as we thus establish the set of denotations (what logicians call semantic rules), we cease to deal with a formal system that is neither true nor false but only logically consistent. We come instead to deal with a physical theory that may, or may not, prove sufficient to impose a rational order on elements of our experience. I mark this transition by calling the abstract system studied by mathematicians the "Euclidean formalism," while calling the "Euclidean theory" that aggregate of formalism with semantic rules the nonmathematician takes for granted when he speaks of "Euclidean geometry."

In the Euclidean theory the derived theorems are rendered colligative relations by virtue of the denotations attaching to their conceptual terms. These denotations, though designated "semantic rules," cannot of course be reduced to any hard set of explicit rules. On the contrary, like the denotations of scientific concepts generally, they reflect the characteristic compromise forever struck among reliability, generality, and simplicity. If we describe a "point" as a $0.5 \mathrm{~mm}$ pencil spot on paper we give a semantic rule adequate for the high-school student of geometry. But for the machinist this is much too broad a "point"; and for the engineer concerned with the guidance of long-range missiles this is much too narrow a "point" (for him a "point" may be several miles in diameter).

As in so many other cases, our grasp of meaning is secured by our sense of purpose. We carry in our mind's eye some image of the "ideal" entities of a Euclidean theory in which the theorems are simply truths by definition. We thus frame conceptual models against which we measure elements of our experience which afford us only approximate cognates of points with no parts, lines with no breadth, solids with no deformability, etc. For the purpose in hand we may or may not judge the approximations "close enough" to proceed by the methods of Euclidean geometry. And, if we do so proceed, our predictions may quite well fail if we have erred in judging what are adequate approximations to the ideal entities of the Euclidean theory.

By and large, predictions drawn from the colligative relations accommodated in the Euclidean theory are excellently borne out. The Euclidean theory thus proves sufficient to the test of experience, but this rather mild adjective is the strongest justifiable. To be sure, if we attach the same set of semantic rules to most of the non-Euclidean 
formalisms we arrive at colligative relations that fail the test of experience. But a properly constructed Riemannian geometry eventuates in colligative relations that, in the range of terrestrial magnitudes and to the sensitivity of terrestrial measurements, yield the same predictions we draw from the Euclidean relations. Thus, in terrestrial applications Euclidean geometry is not forced on us but, rather, preferred by us for its superior "simplicity." Euclidean geometry is then again typically a scientific theory, judged for sufficiency like any other scientific theory.

Euclidean geometry highlights an important point characteristic of scientific theories generally, but not elsewhere always as evident as here. Always a scientific theory is the aggregate of a formalism and a model. The formalism constitutes the deductive machinery required for the theory's function as a correlative device; the model gives rise to the multitude of semantic rules and, as we will see, much more besides. In view of this conclusion, my account of scientific theories will proceed by way of separate treatment of formalisms on the one hand and models on the other. But we must keep well in mind that important interactions between model and formalism must be allowed for when, as we will, we come to theories superficially very different from Euclidean geometry.

\section{Logic and Mathematics}

With reference to the basic notion of congruence, Poincaré comments that "if there were no solid bodies in nature, there would be no geometry." Thus greatly conditioned by experience, formal geometry is however not at all concerned with experience: it tells us nothing whatever about solid bodies, or anything else, to be found in the world. Affiliating semantic rules with the formalism, we enter the realm of the physical scientist who, however indirectly or approximately, must always somehow render account of the world of experience. But precisely as we make that affiliation we part company with the geometer, and quit the realm of strictly formal disciplines. Profoundly involved in all scientific advance, and substantially influenced by such advance, pure logic and pure mathematics are obviously not themselves sciences.

Formal logic imposes certain syntactic rules for the combination and transformation of sets of symbols that never represent anything 
more than particular marks on paper. Whether premises and conclusions are "true," in the sense of corresponding to something in experience, is quite emphatically not the concern of pure logic-the sole concern of which is to ensure that a conclusion is a conclusion, formally necessitated by the premises. The classical syllogism, for example, is a form devoid of content and, more generally, the formalisms of logic involve only vacant relations. Indeed, the necessity of the conclusions drawn from such formalisms entails that vacancy. Logicians then quite wisely decline the scientist's endeavor to develop useful applications of their formalisms. For to secure such applications the scientist must identify the symbols of the formalism with concepts having experiential denotations. Just as with geometry, the logically necessary conclusions are then rendered statements about experience that experience may or may not confirm.

Some very simple illustrations can easily be given in the case of mathematics where, Einstein suggests, an entirely similar situation prevails.

Mathematics deals exclusively with the relations of concepts to each other without consideration of their relation to experience.

Consider this elementary statement of arithmetic:

$$
1+1=2
$$

One stone plus another stone gives two stones; one apple plus one orange gives two pieces of fruit; and so on. Is pure mathematics then teaching us something about experience? Certainly not! We have identified the abstract symbols with "things" we already knew conformable with the stated relation. Lacking such prior knowledge, we are not always so fortunate. One barely subcritical mass of plutonium plus another such mass do not yield two units of mass, but a nuclear explosion with products having less than two units of rest mass; one quart of alcohol plus one quart of water do not make two quarts of fluid but something less; one rabbit plus another rabbit. . . . Plainly, in this second group of instances, predictions founded on the arithmetic statement may fail of confirmation.

Assuming the formalism of simple algebra, we readily agree that $(60)(1)=(1)(60)$. But it does not follow that if 1 man can dig a post-hole in 60 seconds then 60 men can dig that hole in 1 second. Or consider a less facetious case: 


$\begin{aligned} \text { If } A & =B \\ \text { and } B & =C \\ \text { then } A & =C\end{aligned}$

Given the premises, we may readily agree that it is necessarily the case that $A=C$. Assigning denotations to the abstract symbols, we now let $A, B, C$ represent the "weights of objects"; and we let the symbol = stand for "equal within the sensitivity of the best available balance." With the assignment of these denotations it is no longer necessarily the case that $A=C$. For example, suppose that $A$ is lighter than $B$ by an amount barely less than the sensitivity of the balance, and that $B$ is lighter than $C$ to just the same extent. Then, although $A=B$ and $B=C$, when we compare $A$ and $C$ we find $A$ clearly lighter-so that $A \neq C$.

Such contingency we do not ordinarily associate with logic and mathematics, but of course it is not logic and mathematics that then fail. We left the certitudes of logic and mathematics behind us when, to acquire a scientifically meaningful statement, we invested the formalism with experiential relevance. No longer vacant, the formally impeccable conclusion is now no longer necessary. Benjamin Peirce defines mathematics as "the science which draws necessary conclusions." But the necessity of the conclusions arises precisely because, as Peirce's distinguished son Charles Sanders well recognized, mathematics is not a science.

The seductive misconception that mathematics is a science may, on occasion, prove very actively misleading. Thus, for example, consider that we discover some elegant formal demonstration that $B$ "follows necessarily" from a set of premises including $A$. Identifying $A$ and $B$ with certain concepts, we find that $B$ represents a known colligative relation. Betrayed by the thought that mathematics teaches us something about the world, we may then fall prey to such age-old fallacies as affirmation of the consequent: finding $B$, we conclude that $A$ certainly exists. Incredible as it may seem, competent scientists have been so deluded. A celebrated instance developed from Newton's demonstration that:

If the density of a fluid which is made up of mutually repulsive particles is proportional to the pressure, the forces between the particles are reciprocally proportional to the distance between their centers, And vice versa, mutually repulsive particles, the forces between which 
are reciprocally proportional to the distance between their centers, will make up an elastic fluid the density of which is proportional to the pressure.

Newton's insight was far too penetrating to leave him deceived about the significance of any such demonstration. He explicitly called attention to its status in the following qualifying statement:

But whether elastic fluids do really consist of particles so repelling each other is a physical question. We have here demonstrated mathematically the property of fluids consisting of particles of this kind, that hence [natural] philosophers may take occasion to discuss that question.

I have italicized the two words making the key distinction, so obvious one might think it hardly worth mentioning. But the force of the qualification was entirely lost on many of Newton's successors. Given that gases do conform to Boyle's law, they promptly concluded that Newton had proved the corpuscular nature of gases. This was certainly the opinion of John Dalton, to whose intelligence we owe the creation of what has become the modern atomic theory.

The line I draw between science and pure mathematics has of course been crossed repeatedly, and in both directions, by such as Archimedes, Newton, Laplace, Gauss, Bessel, Eddington, etc. Ordinarily, however, the modern scientist need not and does not cross it. In the immense stockpile of fully developed formalisms he usually can find at least one competent to correlate his subject matter. The antecedent explorations of logicians and mathematicians then at once make him master of all the formally "necessary" consequences arising from adoption of any given set of abstract premises. An Einstein, Born, or Heisenberg thus acquires completely worked-out systems of non-Euclidean geometry, matrix algebra, tensor calculus, etc. The extent to which such formalisms have successfully been emptied, or divested, of experiential associations becomes now the measure of the generalized applicability for which they are prized by scientists. Because the formalisms are vacant, we can give them whatever content we please and examine the consequences. We associate concepts having experiential denotations with the abstract terms appearing in formal relations, and in systems of formal relations-and so acquire the potential scientific laws and theories from 
which, as Weizsäcker observes, we learn something about the world of which logic and mathematics as such say nothing.

A real path of light is for a living, observing man a road of wonder; a possible path of light is a geometrical curve and nothing more. But the "possible things" are on the other hand necessary instruments of thought. For since we do not know in advance the full truth, we can think of the real only by separating it out from the plenitude of the possible.

Abstract formalism and scientific law. My primary concern is the role of formalisms in co-ordinating scientific laws in theories. I approach this matter by way of very brief consideration of the second major scientific application of logic and mathematics, in the discovery and expression of such laws. Weyl comments that:

... Kepler's astronomical discovery would have been impossible without the Greek geometer's preceding discovery of the ellipses as a mathematically simple class of curves.

In modern times the idea of quanta first occurs to Planck only after he had expressed the data on blackbody radiation in a formula he could not have obtained had he known nothing of exponential functions. Beyond thus furnishing (1) essential expressions for our laws, logic and mathematics provide (2) techniques for the analysis of our data. Whether the analyses are as sophisticated as those required of Kepler and Planck, or as simple as that required to discover Boyle's law in Boyle's J-tube data, such analyses are clearly indispensable.

Closely related to the last function of abstract analysis, observe that it gives us (3) capacity to calculate empirically inaccessible "data" that may manifest some significant regularity. I explicitly distinguish "data" from data: "data" are inferences drawn, from data observed, with the aid of previously established colligative relations and implicit invocation of the principle of continuity. As an example consider that, like Cannizzaro, I suspect and seek some regularity in the relative densities of various gases under the same conditions of temperature and pressure. For practical reasons (involatility, instability, etc.) all the densities cannot be measured under the same conditions. I then make my measurements at different temperatures and pressures and, applying Boyle's and Charles' laws, mathematically reduce my data to "data" allegedly representative of some one stand- 
ard condition. In "data," but not in data, a significant small-wholenumber relation is then easily brought to light. Last, and only marginally distinct, is (4) the abstract analysis yielding "data" unobserved but not in principle unobservable. Thus, for example, from measurements of motion along planes set at various angles to the horizontal, Galileo extrapolates to the limiting case of a plane set at $90^{\circ}$ to the horizontal. The acceleration calculated for this case he equates with that of the "free fall" he was unable to study directly. IVe find this "datum" a crude but not unreasonable approximation to the datum our superior experimental tools bring within easy reach.

The formal disciplines lend the scientist a support clearly necessary and, equally clearly, insufficient. As earlier implied (p. 178), every scientific application of formal analysis has behind it (and sometimes almost concealed by it) irreducibly physical ideas. No formal argument but a physical hypothesis emboldens Galileo to extrapolate through the discontinuity unavoidable in passing from rotary motion along a plane to the non-rotary motion of "free fall." The formal analysis conducted by Cannizzaro is inspired and directed by a physical concept of corpuscularity. We cannot arrive at Boyle's law without the physical concepts of pressure and volume, or at Kepler's laws without the concept of heliocentric orbit. Whence derive these physical concepts? From “induction"?

\section{INDUCTION}

What we may mean by induction is not a little obscure. Is it simply the process by which we pass from particulars to the general? But the conditioned reflex already manifests capacity for such "induction." Trained by repeated electric shocks to turn to left and not to right whenever two tracks are open to him, has the earthworm then "reasoned inductively"? Surely the grandiose concept of "scientific induction" must involve more than straightforward generalization based on the principle of continuity.

By familiar processes of deduction we reason our way from theoretical postulates to general colligative relations accommodated in that theory, and, further, to particular predictions drawn (for particular circumstances) from those relations. I think that induction is for most of us the hypothetical inverse process by which we reason our way from particular observations to general relations, and, further, to the postulates of the theory accommodating those relations. 
That we can imagine such a process, and have a name for it, does not of course ensure its existence. Admittedly, Newton implies that his theoretic postulates were obtained "by induction." But, after more than a quarter of a millennium, we have yet to discover a formal method competent to produce, from Newton's data, Newton's concepts and postulates. We observe, too, that the Newtonian postulate of universal gravitation was denounced by Leibniz, and others no fools, as a reprehensible relapse back to invocation of an occult influence as "explanation" of physical appearances. That one man's "induction" should be another man's occultism perhaps suggests that induction involves rather more than formally correct reasoning. Logicians still write books on induction a century after Bernard correctly noted its supra-logical character:

When we believe we advance from a particular case to a principlethat is, when we proceed inductively-we really deduce; the experimenter lets himself be guided by a principle supposed to be provisional, which he modifies at every instant while he searches in more or less darkness.

Logic and mathematics sustain the deductive operation, but are powerless to produce the provisional hypotheses on which all else depends. Such an idea as universal gravitation is not derived but invented! How? Is it not the same Newton that preaches the cautious virtue of induction who also says:

No great discovery is ever made without a bold guess.

"Induction" involved with guessing? What a fall! But, when one comes to think about it, our naïve concept of a formal method of induction cannot but seem absurd, irretrievably negated by experience that, as Cohen points out, is immediately accessible to each among us.

... it is often claimed that the various canons of induction tell us how to discover the causes of phenomena. The reader can readily test this claim by applying these canons to discover some causes not yet known to him, e.g., the cause of cancer, ...

In general, there is no definite method of discovering new truth, ... The discovery of what has hitherto been unknown involves a leap into the dark, and the tragic history of human failure to solve our vital problems shows how real is the darkness. 
The hypothetico-deductive method. Induction is a bipartite process accurately, if excruciatingly, described by the barbarism of the section heading. Certain items of experience that somehow disturb him spur the scientist to invent new hypotheses. Each of these he evaluates by discovering whether, by deduction from it, he can indeed account for the items of experience from which he first took departure. We have then first an act of creation, of some tentative hypothesis, at once followed (and probably overlapped) by an act of appraisal. The first (inductive) creative flight and the second more prosaic (deductive) descent proceed, says Whewell, along the same stairway:

But still there is a great difference in the character of their movements. Deduction descends steadily and methodically, step by step: Induction mounts by a leap which is out of the reach of method. She bounds to the top of the stair at once; and then it is the business of Deduction, by trying each step in order, to establish the solidity of her companion's footing. Yet these must be processes of the same mind. The Inductive Intellect makes an assertion which is subsequently justified by demonstration; and it shows its sagacity, its peculiar character, by enunciating the proposition when as yet the demonstration does not exist: but then it shows that it is sagacity, by also producing the demonstration.

The historian and philosopher Whewell finds this "sagacity" quite "out of the reach of method." The chemist G. N. Lewis writes:

A detective with his murder mystery, a chemist seeking the structure of a new compound, use little of the formal and logical modes of reasoning. Through a series of intuitions, surmises, fancies, they stumble upon the right explanation, and have a knack of seizing it when it once comes within reach.

Einstein speaks for many distinguished physicists when he declares that:

. . there is no logical way to the discovery of these elemental laws. There is only the way of intuition, which is helped by a feeling for the order lying behind the appearance.

If the mathematician Poincaré is right, something of the same sort is true even within the domain of the formal disciplines:

Logic has very little to do with discovery or invention. 
Pursuing the chimera of "scientific induction," we are heartily dissatisfied with vague allusions to "sagacity," "knack," "intuition," or "feeling for order." But how else can one define the imaginative insight required for the greatest conceptual advances in science-demanded, for example, first to see sameness in the fall of leaf and apple, and then in fall of apple and moon? The multitude of such insights achieved by certain individuals, and wholly denied to contemporaries amply skilled in logic and mathematics, forces us to recognize how much more than those skills must enter into the faculty most esteemed by scientists. To be sure, "imaginative insight" is an unobservable, perhaps a fiction, and pronouncement of these words solves no problem. But "logical induction" is also strictly hypothetical, an unobservable, a fiction-and a thoroughly dishonest fiction at that. When we say "imaginative insight" we admit that the creation of new ideas poses an unsolved problem, and we point to certain (psychological) areas in which at least a partial solution may be sought. But when we say "logical induction" we make solution impossible, either by denying the problem with a pretension to having solved it, or by pointing in the wrong direction.

Preparing the way for an intuition they themselves cannot supply, logic and mathematics have some role even in the first stage of the hypothetico-deductive operation; and they are of course centrally involved in the second stage of appraising the fruits of intuition. Some prove not peaches but lemons. The root of the progressivism of science was earlier identified with the negative capacity for reasonably prompt rejection of error reasonably convincingly exposed: logic and mathematics constitute an essential part of the machinery of rejection. No "Canons of Induction" can yield us a first conception of a "true cause." But Mill himself stresses that such Canons are primarily methods of elimination and, as part of the informed common sense of scientists, Mill's Canons quite efficiently indicate the hypothetical causes we do well to reject.

To the pure logician and the pure mathematician the choice of postulates is in principle essentially free, limited only by the requirement of self-consistency. To the physical scientist that choice, though underdetermined, is closely restricted: for him the only theoretic postulates worth considering are those from which he can derive the known relations for which theoretic accommodation is sought. Just so, Euclid selected his postulates, rather than others with which 
modern geometers concern themselves, because his postulates did lead to the relations already known to him.

Galileo's original conception of free fall, as uniformly accelerated with increasing distance traveled, is formally unassailable. But only his second conception, of a motion uniformly accelerated with increasing time traveled, proves compatible with what his experiments showed to be the case in nature. Newton might perfectly well have postulated an inverse first power law of gravitation. The combination of this with the other Newtonian postulates might have led to a system formally wholly unobjectionable, but certainly not to an acceptable theory of celestial dynamics: this system does not yield theorems identifiable with Kepler's laws, which were already known. The choice of the familiar inverse square law, on the other hand, at once yields a convincing derivation of just those laws. Thus "intuition" comes under the control of experience through the mediation of logic and mathematics-in very much the fashion Newton himself suggests.

In mathematics we are to investigate the quantities of [hypothetical] forces with their proportions consequent upon any conditions supposed; then, when we enter upon physics, we compare those proportions with the phenomena of nature, that we may know what conditions of those forces answer to the several kinds of attractive bodies.

"Induction" represents the co-ordinated function of imaginative, formal, and empirical components prettily displayed in Rheticus' account of the procedures of his revered master, Copernicus.

. . . he assumes new hypotheses, not indeed without divine inspiration and the favor of the gods; by applying mathematics, he geometrically establishes the conclusions which can be drawn from them by correct inference; . . .

These conclusions were then compared with the data, Rheticus telling us that:

. . my teacher always has before his eyes the observations of all ages together with his own, assembled in order as in catalogs; . . .

\section{DEDUCTION}

The whole conception of scientific theories as postulational systems assumes the availability of effective machinery of formal de- 
duction. Ordinarily quite simple and familiar machinery serves our needs. Only the theories of modern physics, chemistry, and astronomy require elaborate techniques of modern mathematics that are neither less, nor more, rational by virtue of their sophistication and unfamiliarity.

Throughout the discussion of induction I took deduction for granted. Indeed, we all do seem to share the same reasonably clear idea of what is meant by deduction. Moreover, unlike induction, deduction does seem reducible to methodical operations in accordance with definable (syntactic) rules. This appearance is not wholly deceptive. But Polanyi's analysis of an opinion of Poincarés rather strongly suggests that even completely formal deduction is not free from all complications.

To look at a mathematical proof by merely verifying each consecutive step-says Poincaré-is like watching a game of chess, noting only that each step obeys the rules of chess. The least that is required is a grasp of the logical sequence as a purposeful procedure: what Poincaré describes as "the something which constitutes the unity of the demonstration." It is this "something"-perhaps in the form of an outline embodying the main steps in the proof-for which the student will grope, if baffled by a sequence of operations which convey no sense to him, and it is again this outline, embodying the general principle or general structure of the mathematical proof, which will be remembered when the details of the proof are forgotten.

The "something" no doubt involves an element of familiarity. But, in view of Jeffreys' comment, it seems to me that the competent mathematician must bring that something even to formal operations unlike any he has before attempted.

Deduction cannot be completely formalized, as was shown by Lewis Carroll in "what the Tortoise said to Achilles". . . .

. . . even deductive logic needs a nuotion of appreciation that cannot be formally expressed within the system, but depends on looking at it from outside; . . .

The "notion of appreciation" that escapes expression even in completely formal systems looms still larger in our use of scientific theories. There, however, the "notion" may perhaps be more narrowly definable. Let us now abandon the useful but arbitrary absolute dichotomy hitherto maintained between the formalism and the 
model present in each scientific theory. For, beyond semantic rules, we draw also from the model the notion of appreciation that gives us full command of the formalism. Consider for example that, though presumably we might have been, in secondary school we were not taught the abstract formalism of the modern geometer. Instead we were taught Euclidean geometry with the aid of diagrams and real or imaginary constructions. The geometric formalism was thus supported by models, e.g., when the concept of congruence was represented to us as the physical superposability of two rigid figures. We could then easily "see" our way through relatively complex proofs because we had in view, sometimes with the physical eye and always with the mind's eye, a relevant physical situation. From physical conceptions entirely foreign to the geometric formalism, we thus acquired the notion of appreciation we used in producing workmanlike Euclidean proofs from Euclidean axioms. And this is but a familiar special case of an important general situation. The work of Bruner and his associates provides convincing evidence that humans reason better when they work with concrete materials rather than with abstract logical symbols presented in propositions of identical form.

. . . in the concrete realm verisimilitude provides a way of checking upon validity. . . .

... much of human reasoning is supported by a kind of thematic process rather than by an abstract logic.

It is the model that supplies verisimilitude and evokes the thematic process that supports our operations with the formalism of a scientific theory.

Given the importance of this thematic process, the absolute dichotomy of syntactic and semantic rules becomes quite as indefensible as the related dichotomy of formalism and model just now abandoned. And, beyond any vague "thematic process," the model offers the formalism several more specific elements of support. Thus, for example, in making derivations from theoretic postulates, often we simply drop out certain terms, or groups of terms, we hold negligible. What suggests and justifies these approximations? Nothing in the formalism as such but, rather, considerations of relative magnitude that can arise only as we impute "meaning" to the abstract terms of the formalism.

Even when we need make no approximations, our use of the syn- 
tactic rules of the formalism seems always affected by our awareness of the semantic rules we draw from the model. No scientific theory meaningfully considered as such has yet been completely formalized, and it is much to be doubted that any such theory ever will be. Quite generally then we find, in making our derivations, that nothing in the formalism supplies, or even implies, what we need to pass from one step to the next. We make that passage all the same, using assumptions borrowed, on the strength of the model, from other branches of science or from common sense. When the need for such assumptions is recognized, they are made explicit. Probably more often the assumptions are only implicit, wholly unmentioned because the principles we take for granted make them so completely "self-evident." Thus, for example, the modern professional geometer easily shows that Euclid's proofs require assumptions underivable from the postulates he states. As nonprofessional geometers we make all the requisite assumptions implicitly, even as Euclid did. Given the model that makes the Euclidean theory, the assumptions become so "obvious" we easily see our way to and through them. But, precisely because they are so "obvious," we fail entirely to see them.

A conceptual device of immense power, the physical model is inseparably associated with a grave hazard. The very thematic process which so strongly sustains us may also, on occasion, betray us. Thus Bruner notes that when we reason in concrete rather than abstract terms we are predisposed toward certain conclusions that have for us an air of verisimilitude "in spite of the fact that they are both incorrect logically and readily detectable as such when they appear in a neutral form." We proceed to those expected conclusions by way of implicit assumptions wholly uninspected because their presence is wholly unsuspected. A substantial check to progress may thus develop: long years may pass before anyone discovers the implicit assumption that, once recognized as such, simply will not bear inspection. Some have thought to bypass this hazard, by giving scientific theories a completely explicit formal development-reaching its optimal expression in the "purely mathematical" scientific theory that involves no physical model. Is this a program to be taken seriously? A multitude of historical precedents suggests the invariable presence of implicit assumptions in scientific theories. Moreover, as we might by now suspect, the whole idea of a purely mathematical scientific theory is itself delusory. 


\section{Models}

Qualitative differences in the models on which they are founded will make scientific theories notably different in appearance. Beginning with those theories that do indeed seem "purely mathematical," let us consider the adequacy of Bridgman's very persuasive analysis of their status.

Formerly mathematical theories usually had in the background a physical model of some sort, as, for example, the kinetic theory of gases had in the background a model consisting of idealized molecules, . . . The molecules were so simplified that they were amenable to mathematical treatment. We had then a sort of double theory-a mathematical theory of the idealized model, and then a physical theory consisting of the statement that there was a correspondence between the idealized model and the actual physical system sufficiently close so that certain properties of the physical system were reproduced by the model. The point in making such an idealized physical model was that it had a mathematical theory simple enough to be handled.

It presently appeared to reflection, however, that there was an unnecessary step here-since all that could be done in any event was to set up certain correspondences between the results of the mathematical manipulations and the physical system, why have the intermediate step of the idealized physical model, since a correspondence to a correspondence is also a correspondence? This of course is exactly what has been done in recent wave mechanical theories, particularly that of Dirac, and we know that such a procedure has been brilliantly successful. I think that the reason that this change of attitude was so long deferred was that it was not realized that there was an intermediate step- . . the model was actually identified with the physical system.

What we now have is in effect mathematical models rather than physical models . . .

Can a mathematical model thus replace the physical model? A strictly formal system, we saw earlier, is characterized primarily by its vacancy. Were scientific theories purely mathematical, how could they at all refer to experience?

Bridgman considers the physical model an "unnecessary step" because "a correspondence to a correspondence is also a correspond- 
ence." In the formal realm we find multiple abstract theorems in logical correspondence with abstract premises. In the physical realm we find multiple colligative relations which, by virtue of the denotations attaching to their concepts, stand in correspondence with elements of experience. But where is the correspondence between these correspondences? How can we pass from formal theorem to physically relevant colligative relation? Clearly we must identify some of the abstract symbols with indicative concepts. Even a quite vague implicit model may still furnish clues sufficient for this identification, and it is thus that the model becomes the link between theory and experiment. But with no physical model there is no link, and we can then find in a purely mathematical construction no scientific theory. Thus, Hutten emphasizes, quite aside from their well-known psychological values (and dangers), models have also an indispensable logical function: from them alone we draw the semantic rules that first give some physical interpretations to what are otherwise only formalisms.

... it is often said that the model may be abandoned after it has served its [heuristic] purpose. This would be so if our theories were given as formalized systems and if we could state explicitly all the rules necessary for determining the meaning of any sentence within the theory. In fact, this cannot be done for any present-day theory, and so we need the model also for climbing down the [deductive] ladder to reach the experiment. We may know all the mathematics needed for quantum mechanics, but its interpretation cannot be said to be complete, in the strictly logical sense of this term; and how about meson theory?

Dirac's quantum mechanics, cited by Bridgman, is the generally cited paradigm of the formalism that functions as a scientific theory. But, in introducing his theory, Dirac himself emphasizes not the formal but the physical ideas.

... the mathematics is only a tool and one should learn to hold the physical ideas in one's mind without reference to the mathematical form.

The better to grasp such physical ideas, we always seek models or analogies. And to be sure, Hesse finds something of the sort even in Dirac's highly formal theory. 
Although the theory of observables appears to be a theory about measurements, it is still far removed from measurements as they are actually carried out, and presupposes a particle analogy which is not directly given in experimental data.

The analogy invests the formalism with experiential relevance, and so makes of it a scientific theory; the analogy allows us to hold the physical ideas in mind, and so makes it possible for us to use that theory. Dirac's theory, classic exemplar of the scientific theory "purely mathematical," is nothing of the sort.

Model implicit in formalism. When we look at Dirac's quantum mechanics we nowhere find explicit reference to a model. How then does a model or analogy come to be associated with a theory that offers as overt premises only a set of equations? It is entirely a matter of our previous experience with equations of related types. Given that experience, we at once associate with the formalism a model or analogy founded on the system(s) to which we have earlier applied such equations. A model or analogy is then implicit in the formalism.

Like Dirac's system, Schrödinger's wave mechanics appears at first sight a purely mathematical construction. By now we know that must be an illusion, and in this case the analogy is utterly inseparable from the wave equation just because, as Hutten says,

. . . whenever we see a certain differential equation of the second order (in the space and time co-ordinates), we think of waves, . .

Is what we are thus led to think of any importance? Schrödinger stresses the centrality of the extra-mathematical character of his theory, in terms very similar to those we found Dirac applying to his.

. . I I do not refer to the mathematical difficulties, which eventually are always trivial, but rather to the conceptual difficulties.

The "probability waves" of an electron are quite unlike any familiar waves, but the analogy posed by the implicit wave model is still sufficient to permit us to master those "conceptual difficulties."

A "purely mathematical" system can, then, function as a genuine physical theory, if its equations imply to us something not purely mathematical. Hutten's summary seems entirely just.

There are . . no mathematical models in physics: the equation by itself is not the model. The wave equation is a model only because 
we know it to represent the spreading of a wave through space. It is the interpretation which is attached to the equation due to previous application that we need for describing our experiments.

This reference to "previous application" explains why to the uninitiated quantum mechanics appears a purely mathematical system: unaware of any previous application of like formalisms, they fail to detect the model implicit in the formalism. The same reference explains also how some of those professionally concerned with quantum mechanics can still misconceive it purely mathematical: taking the previous applications and the implicit interpretations entirely for granted, they do not realize how much is thus conveyed to them.

Formalism implicit in model. Until quite recently the vast majority of all important scientific theories have been founded on the conception of some physical model, and make no explicit reference to any formalism. Often syntactic rules may well pass unmentioned bécause the theory requires, and simply assumes, no more than the simplest kinds of everyday reasoning. But even when more elaborate formal operations are involved they may be given little or no overt notice. How then do we acquire the formalism needed to complete the theory? The answer is foreshadowed by our earlier discovery that the apparently purely formal scientific theory always conveys an implicit model. We now encounter the complementary case: the scientific theory that apparently proposes nothing but a model actually conveys an implicit formalism.

Consider, for example, the Mendelian theory which postulates "genes" as carriers of hereditary traits. The appearance, or nonappearance, of each trait in a given organism is then supposed to arise from the random combinations and recombinations, in its ancestors, of allelic forms of some particular gene. The genes are completely hypothetical invisible entities, conceived on the model of "discrete objects." From that model the Mendelian theory at once acquires a formalism-precisely that earlier found applicable to statistical description of random combinations in certain systems of discrete objects. Given the Mendelian model, we are thus led to set up certain axiomatic relations we expect to apply to the independent assortment of genes and, in reasoning from these axioms, we follow the (syntactic) procedures earlier found appropriate.

This example is noteworthy only in its comparative simplicity, for even a very primitive model may suffice to suggest a very highly 
sophisticated formalism. As an example, consider that since Mendel's time we have learned to conceive genes as incompletely independent. We think now of groups of genes associated in linear arrays of some stability (chromosomes). Given this model, Gamow brings to the study of genetic "coding" a very refined formalism already known to permit determination of the statistical distribution of length in the pieces obtained by repeated random breakage of, say, a great many sticks.

Conceiving analogy between two systems, we found on the more familiar a model for the less familiar. So doing, we promptly apply to the second the formalism our previous experience shows successfully applicable to the first. Often we cannot simply adopt that formalism unchanged: instead we purposively adapt it, to allow for the difference(s) that distinguish the problem system from the model system. We meet this situation in the theory Torricelli constitutes by suggesting that the atmosphere be conceived on the model of a "sea of the air." We then conceive the possibility of explaining certain puzzling observations as aerostatic phenomena, analogous to simple hydrostatic phenomena already amply familiar. But now in constructing the axioms of our new theory we take care to modify the axioms of hydrostatics-to render account of that ready compressibility of the atmospheric fluid which so markedly distinguishes it from fluids treated in hydrostatics.

\section{MODELS AND SEMANTIC RULES}

A scientific theory may be constituted by affiliating model and formalism in any of three rather different fashions. Without insisting on their absolute distinctness, I think it useful to distinguish these three cases, which are characterized by the different ways in which "meaning" is attached to the primitive concepts, i.e., those that figure in the axioms of the theory.

First case: Direct denotations and simple models. Consider Newtonian mechanics in its most familiar applications to medium-sized terrestrial systems. Like Euclid before him, Newton gave his system a notably formal development. His primitive concepts include "force," "mass," "time," and "space." In medium-magnitude terrestrial applications we ordinarily attach to "space" denotations established with the aid of meter sticks, systematic triangulation, and the like; to "time," denotations established around clocks of one sort or 
another. "Mass" is perhaps measured, relative to a standard, with an equal-arm balance; and "force" is measured with this same instrument or with elastic systems that obey Hooke's law. Here then Newton's system gains its experiential relevance, as does Euclid's, in the simplest way imaginable: through the attachment of denotations to its primitive concepts, and thence automatically to all derivative terms and relations.

For his primitive concepts Euclid furnished definitions wholly insufficient to establish the working denotations. Newton did exactly the same. The inadequacy of his definitions of absolute time and space was recognized long before relativity theory came to underline that inadequacy. Mass he defines as the product of density and volumewhich is an obvious and immediate circularity since we need the concept of mass to define density. We have then no denotation for mass and-since Newton's definition of force involves the concept of mass-that definition equally fails to establish a semantic rule. How then are the denotations of these concepts established?

Euclid's explicit definitions do not themselves supply denotations, but they are sufficiently suggestive to lead us toward models from which we draw the requisite denotations. Newton's definitions function in exactly the same way. We may, for example, conceive space and time in the sensorium of divinity as somewhat analogous to our own awareness of visual space and subjective time. Force we may conceive as somehow related to the muscular. effort we experience from both the producing and the receiving ends. And mass we may conceive, as did Lavoisier, Laplace, and Newton himself, as something to do with the "quantity of matter." Grasping these ideas, we are led to the working denotations indicated above. We read Newton's definitions intuitively, as we do Euclid's, and extract something they do not themselves contain.

Why didn't Newton state the working denotations explicitly, as semantic rules? Simply because, as has been emphasized repeatedly, any rigid specifications of the denotations of concepts must invariably restrict their applicability. This point emerges in high relief from a very painstaking analysis that brings Braithwaite to the following conclusion:

It is only in theories which are not intended to have any function except that of systematizing empirical generalizations already known that the theoretical terms can harmlessly be explicitly defined. A the- 
ory which it is hoped may be expanded in the future to explain more generalizations than it was originally designed to explain must allow more freedom to its theoretical terms than would be given them were they to be logical constructions out of observable entities.

Newton sought for his system applications far beyond the familiar realm of molar terrestrial phenomena. And so of course he does not shackle his concepts with explicit denotations applicable only in that realm. One cannot expect to determine the mass of an atom with the equal-arm balance, or to apply a spring balance to measure the force of attraction between earth and moon. In its macrocosmic and microcosmic applications the Newtonian system takes on a quite different aspect.

Second case: Indirect denotations and simple models. The "given" in celestial dynamics is represented by certain directional co-ordinates at which a certain planet, say, has been seen at certain times. From these we calculate, by the methods of the formalism, various distances, velocities, accelerations, and the like; and from these, finally, we may calculate the masses of the planets and the forces acting upon them. The Newtonian system is here linked with experience only by way of its derivative concepts: the semantic rules attach to these and not to the primitive concepts. Indeed the primitive concepts are now in some sense unobservables: we no longer measure force and mass with any directness but instead infer their values from other quite different values we do measure.

In its macrocosmic applications Newtonian dynamics thus takes on something of the appearance of quantum mechanical theories. For in these also the primitive concepts are unobservables only very indirectly evaluable, through the medium of the theory and the implicit definitions constituted by its axioms. Whatever the similarity, however, there is also a striking difference: in celestial dynamics we find the primitive concepts readily conceivable. Newton's explicit definitions of his primitive concepts are here just as inactive as in the domain of molar terrestrial phenomena: they do not themselves supply the working denotations. But they are also just as active here as there: they lead us to conceive the analogies and models from which we draw the working denotations-which here we attach to the derivative terms in the formalism. And, in terms of these models and analogies, we find we can represent even the primitive concepts in concrete models and diagrams, and have always the sense of know- 
ing what they mean. Certainly the symbol $F$ in the calculation of a lunar orbit has only the most tenuous association with our muscle sensations and experience of springs. Yet we do conceive analogy here, and we grasp $F$ in terms of the comparatively irrelevant sensations and experience.

In the microcosm Newtonian mechanics functions as in the macrocosm. The primitive concepts may now refer to entities totally unobservable. In the kinetic theory of gases we do not observe molecular velocities, but infer them, perhaps from measurements of density and pressure. We do not measure the mass of a molecule, but infer it, perhaps by way of observations of the Brownian motion of much larger particles. Again the effective semantic rules attach to derivative concepts, such as pressure. Again Newton's explicit definitions do not supply those rules, but again they lead us to the conception of simple models from which, as before, we find it easy to draw the rules. And we find entirely parallel situations in many other theories involving microcosmic unobservables. Mendel's conception of genetic factors and Kekulés conceptions of valence bonds stipulate unobservables we can rather easily grasp in terms of fairly straightforward models. But we must now look at other microcosmic theories in which use of such models is no longer possible.

Third case: Indirect denotations and hierarchic models. Dirac prefaces his exposition of his theory with the following explanatory statement.

We introduce certain symbols which we say denote physical things such as states of a system or dynamical variables. These symbols we shall use in algebraic analysis in accordance with certain axioms which will be laid down. To complete the theory we require laws [i.e., identifications, or semantic rules] by which any physical conditions may be expressed by equations between the symbols and by which, conversely, physical results may be inferred from equations between the symbols. A typical calculation in quantum mechanics will now run as follows: One is given that a system is in a certain state in which certain dynamical variables have certain values. This information is expressed by equations involving the symbols that denote the state and the dynamical variables. From these equations other equations are then deduced in accordance with the axioms governing the symbols and from the new equations physical conclusions are drawn. One does not anywhere specify the exact nature of the symbols employed, nor is such specification at all necessary. They are used all 
the time in an abstract way, the algebraic axioms that they satisfy and the connection [semantic rules] between equations involving them and physical conditions being all that is required. The axioms, together with this connection, contain a number of physical laws, which cannot conveniently be analyzed or even stated in any other way.

The symbols are never defined, yet they have for us some physical signification. How do they acquire it? Concluding a careful analysis of Dirac's theory, Hesse asks:

What then is the significance of the concepts in Dirac's theory? The answer is clearly to be found in terms of the classical analogy. It is this that gives meaning to the purely formal statements of the hypothesis and hence gives rules for manipulation of the concepts. Dirac's discussions about measurement and observability become meaningful if we realize that he has in his mind, not practically possible experimental measurements, but a highly idealized system of particles like those considered in classical dynamics, only with the difference that complete information about the positions and momenta of the particles cannot be obtained . . .

No simple particle model can possibly represent what Dirac's theory seeks to convey. But let me now try to show how a hierarchic model can, and does.

From the extreme abstraction of the formalism that offers neither overt model nor direct denotations for its primitive concepts, we work our way back through a sequence of progressively less abstract theories. Without even thinking much about it, we loosely relate the concept of stationary state, in an advanced quantum mechanics, to the far more readily intelligible concept of electronic orbit in the older quantum theory. Thence, presumably, we work still further back, to classical mechanics-and perhaps at last to the simplest common-sense concepts. No one model or analogy here suffices. Only through an entire series of incomplete but overlapping models and analogies do we contrive ultimately to grasp the nature of the primitive concepts. As Hutten correctly emphasizes, the very same chain of successive partial interpretations-terminating in familiar concepts having clear denotations-is also precisely what we need to link up the derivative concepts in the abstract theory with what we actually do and see in our experiments.

The hierarchic model is a somewhat diffuse composite or super- 
position of elements sometimes superficially syncretic. Thus, for example, with an electronic wave function we compute the probability of finding a corpuscular electron in a certain region. We speak and think then in terms of waves and particles quite obviously not the waves and particles of everyday experience, but somehow related to them. The relation we conceive with the hierarchic model, one example of the function of which is described by Hutten with an apt and vivid metaphor.

Physical theory developed by gradually overcoming the limitations of the original model; but this does not mean that . . the original model is completely abandoned. Some connexion with the original model is retained; the model is re-designed and, thus, refined in order to agree better with our latest experiments. What remains is the wave equation, together with a minimum interpretation in terms of experience upon which the application of the equation rests. It is like the grin and the Cheshire cat; the picture of the cat has receded into the background, but knowing that there was once a cat we understand that the residual phenomenon may be interpreted as a grin.

\section{THE "SUPERFLUITY" OF THE PHYSICAL MODEL}

Working with a purely formal system, the logician seeks assurance that its various premises are self-consistent. In like fashion the scientist desires assurance that his theoretic premises are mutually compatible. If Nicod is right, much the same procedure will be brought to bear in both cases:

The discovery of one system of meanings satisfying a group of axioms is always logically very important: it constitutes the proof that these axioms do not contradict one another; and this is the only known proof of consistency.

If all the primitive concepts of a scientific theory have experiential denotations, we may approve the theoretic premises simply as wellestablished colligative relations. But when some or all of these concepts lack such denotations, the self-consistency of the premises can be examined only in the context of the theory as a whole. Consider for example how Planck's constant, appearing in the premises of a quantum theory, also appears, with other "fundamental constants," in many derivative relations (those referring to blackbody radiation, photoelectric effect, emission spectroscopy, and so on). The theoretic 
premises may be quite variously involved in the derivations of such relations which, fortunately, far outnumber the "constants" to be evaluated. Consequently the excellent agreement of all the calculated values for a given constant supplies heartening (if inconclusive) evidence for the self-consistency of the premises. Moreover, beyond this internal agreement, we can often show that the calculated values agree well with others more directly cietermined. Thus, from the Millikan oil-drop experiment we obtain for the electronic charge a value wholly independent of the involvement of that charge in quantum theories.

This mode of analysis has an interest beyond its bearing on the question of theoretic self-consistency. Through it we may be brought to recognize which parts of our model are "superfluous." For example, consider the billiard-ball model on which the kinetic theory of gases was first founded. We find that through the mediation of the theory we can evaluate certain aspects of that model-e.g., from thermal conductivity, viscosity, and diffusion data we derive reasonably consistent values for the diameter of the gaseous particle; from other measurements we learn something of its "hardness," or deformability. But from our theory we can never extract any specific characterization of the "billiard-ballness" of that particle. That is not at all difficult to understand: finding no place in the theoretic premises, this comparison can figure explicitly nowhere in our theory. Indeed, the formal superfluity of the billiard-ball model is made entirely patent by our continued use of the kinetic theory, even though today the billiard-ball atom has utterly dissolved, into a localized concentration of electronic haze.

Ever seeking maximal parsimony and penury of theoretic postulates, we may think it imperative to strip our theories of all such "superfluous" features. Thus Rankine is led sharply to distinguish between two species of scientific theory-the abstractive and the hypothetical-described as follows by Dingle:

Abstraction is the detection of a common quality in the characteristics of a number of diverse observations: it is the method supremely exemplified in the work of Newton and Einstein.

A hypothesis serves the same purpose, but in a different way. It relates apparently diverse experiences, not by directly detecting a common quality in the experiences themselves, but by inventing a fictitious substance or process or idea, in terms of which the experience 
can be expressed. A hypothesis, in brief, correlates observations by adding something to them, while abstraction achieves the same end

by subtracting something.

Surely the distinction is here drawn oversharply. Dingle himself recognizes elsewhere that Newton's conception of universal gravitation is a genuine invention, a "something added," and no simple abstraction. Nevertheless, there does appear to be a real difference between a hypothetical theory, like that involving the billiard-ball atom, and an abstractive theory, like thermodynamics. The element of addition, and "superfluity," looms much larger in the former-as it does in corpuscular theories generally. What can we lose if we reject such "superfluity"?

Campbell correctly observes that:

The explanation offered by a theory ... is always based on an analogy, and the system with which an analogy is traced is always one of which the laws are known; . . . Thus our theory of gases explains the laws of gases on the analogy of a system subject to dynamical laws.

This system hypothetically consists of particles perfectly elastic in their collisions, and such particles and collisions are of course unlike any known to us. The explanation the theory provides thus becomes a function of the extent to which it indicates some reasonable approximation to such particles and collisions. The billiard-ball analogy offers the best possible approximation and, rejecting this "superfluity," we at once lose most of the sense of explanation we find in the kinetic theory. But is this a loss of any real importance? Does the scientific function of a theory have anything whatever to do with the purely subjective feeling of explanation it arouses in us? Duhem answers in the negative, stressing also that the explanations are often the least durable features of theories.

When the progress of experimental physics goes counter to a theory and compels it to be modified or transformed, the purely representative part enters nearly whole in the new theory, bringing to it the inheritance of all the valuable possessions of the old theory, whereas the explanatory part falls out in order to give way to another explanation. . . .

This continuity of tradition is not visible to the superficial observer due to the constant breaking-out of explanations which arise only to be quelled. 
For Duhem the explanations are parasitic upon the "purely representative" function of theories he considered as primarily correlative devices. But is not the "superfluous explanation" in itself a wonderfully effective correlative device? Given our previous knowledge of classical dynamics, the very words "billiard-ball atom" conjure up the major part of the kinetic theory. We imply the axioms and formalism of Newtonian mechanics, earlier found applicable to the collisions of ("ideal") billiard balls. From these we arrive at deduced theorems rendered colligative relations when, on the strength of the model, we identify certain terms with indicative concepts like "pressure." WVe thus acquire the semantic rules we need and, though today the model is quite passé, the memory of that Cheshire cat is still active in eliciting our recognition of what passes as its grin. Beyond all this, the model supplies the notion of appreciation that helps us use the formalism, and suggests also a multitude of auxiliary assumptions often helpful and sometimes indispensable. In certain cases (though surely not in all) some such assumptions may later prove fallacious. Suppose we try to avoid this danger, by seeking a completely explicit statement of premises, syntactic rules, and semantic rules. However "purer" formally, our statement of the theory is now vastly more complex.

Given the model, we readily gain command of the theory to which it gives such compact expression. This situation is, of course, a quite general one. Thus, given our previous knowledge of hydrostatics, the phrase "sea of the air" by itself constitutes an essentially complete aerostatic theory almost infinitely more difficult to express in any substantially formal way. Rather than accept such complications, we ordinarily welcome the support of the "superfluous" model. If it leads us automatically to fill out premises and syntactic rules with implicit assumptions, so much the better. If deductive ladders involve rungs unseen by the eye but unerringly found by the foot, we may use those ladders to good advantage even though, one day, the hitherto unseen rungs crumble under the gaze of eyes newly critical. This will seem a catastrophe only to those who confound scientific theories with ageless truths-but there are some such people.

The positivists" "theory without superfluity." In accordance with the earlier diagnoses of Comte and Mach, Duhem found science weakened by its chronic infection with metaphysics. All thought to create a science resistant to that infection. Such a science will eventu- 
ate in rigorously formal postulational systems designed solely as correlations of economic descriptions (of relations between observables) useful for predictive purposes. Formal construction brings to light implicit assumptions (often metaphysical in nature). Scrupulous attention to observables as such brings to light their usual freight of plausible inference (often metaphysically inspired). These positive enjoinders evoke also a corollary set of negative injunctions. Scientific effort is to be conserved by giving up all search for explanations, impermanent excrescences on positive knowledge, and by eschewing all use of models always potentially delusive.

Duhem's conception of a scientific theory was this:

A physical theory is not an explanation. It is a system of mathematical propositions, deduced from a small number of principles, the aim of which is to represent as simply, as completely, and as exactly as possible a group of experimental laws.

For Duhem thermodynamics was the non plus ultra of a scientific theory. It eschews the superfluity of an overt model; it is strictly descriptive and makes no pretense to offer explanations; and it is superbly efficient as a summary expression of what we already know. Let us then examine the scientific virtues of thermodynamics. Three points will be noted.

First: As a scientific theory, thermodynamics must involve some model, and we need not look hard to find it. Though ultimately we may arrive at a highly formal thermodynamics, constituted by a set of equations involving such symbols as $E$ and $S$, we always travel by way of earlier lessons involving heat engines. Only by virtue of this model are the symbols for the primitive concepts "energy" and "entropy" invested with physical meaning, and the derivative concepts with experiential denotations. Hutten puts this matter clearly and strongly.

It is sometimes said that to choose the heat engine as a model process is due to the industrialization which started in the early nineteenth century when thermodynamics was formulated; or that to make schematic diagrams of such an engine is merely a psychological help in visualizing the thermal processes in nature. This does not explain the fact that it is taken as a standard in terms of which we interpret nearly all heat phenomena, even those that at first sight do not seem to fit this interpretation; and that it shows how terms such as 
"system" and "state" can be used in a statement amenable to an empirical test.

The transformation of energy from one form into another can be understood in terms of this interpretation. Mechanical, electric, magnetic, chemical, and radiant energy produce heat and make the engine run; and all these various kinds of energy can be precisely measured through the mechanical equivalent of heat. The concept of energy becomes more comprehensive but the model shows how to use it, since it can be interpreted ultimately in terms of mechanics.

Second: Formal thermodynamics is a subject found difficult even by very able students. In the absence of suitable overt models, they cannot "see" their way through a thermodynamic derivation as they do through, say, a geometric derivation intrinsically no less difficult. Not only students have trouble with thermodynamics: their instructors do, too! We find textbooks on thermodynamics almost invariably blemished by one or several egregious errors-errors far less frequent in expositions of theories that, though perhaps involving more difficult formalisms, do offer overt models.

Third: A theory conspicuously difficult to master, thermodynamics is also a theory far less fruitful of discoveries than the corpuscular hypotheses so scorned by Duhem, Mach, and Comte. Precisely because the highly formal theory recommended by Duhem lacks all superfluity, it must be a comparatively dull heuristic tool. For consider the observation made, in another context, by Hesse.

. . . one of the main functions of an analogy or model is to suggest extensions of the theory by considering extensions of the analogy, since more is known about the analogy than is known about the subject matter of the theory itself. . . . A collection of observable concepts in a purely formal hypothesis suggesting no analogy with anything would consequently not suggest either any directions for its own development.

We do not make thermodynamics a more effective instrument of discovery by giving it a still more starkly mathematical formulation. I may find a real sense of beauty in Carathéodory's formulation of the second law of thermodynamics, which apparently removes all vestige of reference to physical models. But I do not know of a single major scientific discovery consequent to Carathéodory's work. On the other hand, I know a great many such discoveries originating in Boltzmann's conception of the second law, which at last explains it 
by associating it with an explicit particle model. Thus, for example, Planck comes to his celebrated interpretation of blackbody radiation through this species of thermodynamics.

Is thermodynamics perhaps simply a poor exemplar of the great heuristic power to be found in other theories with the highly formal construction recommended by Duhem? Newtonian mechanics has displayed immense heuristic power, and can be given a thoroughly formal development, but we customarily envision perfectly concrete models whenever we apply the formalism. Classical electrodynamics, in the severely abstract form given it by Duhem and Poincaré, seems a rather more purely mathematical theory. But observe that the breakthrough here was not made by the French formalists but by Maxwell who-taking his departure from Faraday's conception of "lines of force"-derived from a variety of elaborate mechanical models, for the ether, a support clearly essential in the construction of his theory.

What of quantum mechanics, a highly formal theory of great heuristic power? Consider first that the breakthrough to the quantum hypothesis-the work of Planck, Einstein, and Bohr-was powered by the quest for explanation and strongly supported by the use of quite overt models. The subsequent development of more formal theories was then in part a consolidation of a gain already made. To be sure, it is claimed that the sophisticated quantum mechanics of Heisenberg, Dirac, and others proved attainable only because all conjectures about unobservables were suppressed along with all desire for explanation-because the systematic correlation of observables was adopted as the sole legitimate aim of physical theory. But I long ago remarked (p. 44) that the deep concern for observables so evident in modern quantum mechanics is far from being a complete novelty. And Born, who should know, writes:

Heisenberg felt that quantities which had no direct relation to experiment ought to be eliminated. He wished to found the new mechanics as directly as possible on experience. . . it is exactly the fundamental principle of modern science as a whole, that which distinguishes it from scholasticism and dogmatic systems of philosophy. But if it is taken (as many have taken it) to mean the elimination of all non-observables from theory, it leads to nonsense. For instance, Schrödinger's wave function $\psi$ is such a non-observable quantity, ... 
However it may be with the two points noted above, we must come in the end to a third and crucial question. Is the demonstrated heuristic power of quantum mechanics something due to, or achieved in spite of, the highly formal construction of that theory? So posed, the question is not only heretical but superficially absurd. Has not everybody heard of the "mathematical difficulty" presented by Dirac's quantum mechanics that, in Noyes' words,

. . . turned out not to be a difficulty, but a brilliant success for the theory, since such "positive electrons" [postulated to resolve the difficulty] were soon discovered. . . . Thus the mathematical requirements of relativistic and quantum mechanical structure led to a physical prediction which was experimentally verified.

Fewer perhaps have heard that the physical prediction thus drawn from "mathematical requirements" was (1) regarded, by such eminent theorists as Pauli, as an absurd defect of Dirac's theory, and (2) completely unknown to Anderson, who made the actual experimental discovery of the positron. A somewhat similar claim, that the discovery of electron diffraction was uniquely the fruit of de Broglie's original theory of matter waves, is similarly misleading. De Broglie's highly formal theory was indeed a mathematically elegant correlation of many data already known, but Born feels able to write that de Broglie

. . . studied the consequences for plane waves and indicated the interpretation of Bohr's quantum conditions with the help of standing waves. But what did he predict? As far as I know, nothing.

In fact, it was only after Elsasser had seen the suggestive maxima and minima in some of Davisson's electron-scattering curves that the de Broglie wave model was taken literally enough to produce the theoretical prediction of that electron diffraction presently established experimentally by Davisson and Germer and by Thomson.

Noyes suggests that the methodology of modern physics is characterized by its "emphasis on the mathematical structure of the theory" rather than on predictions drawn from models of its "inferred entities." But I would think that no emphasis on mathematical structure as such will yield physical predictions of genuine novelties. Furthermore, and absolutely conclusive in my opinion, there is the clear evidence afforded by the quantum physicists' whole terminology. In 
trying to convey to the novice some idea of potential energy, and activation energy, the physicist may sketch analogy with familiar experience of balls rolling downhill. But, exactly as in the use of the heat engine in the exposition of thermodynamics, this is no trivial pedagogic device. In his research the physicist may investigate potential energy "wells" and "barriers," and he speaks of a "tunnel effect.” Thus, as Hebb quite properly observes:

The worker in the laboratory does not merely report and expound by the aid of analogy; that is how he thinks, also.

Is it not preposterous to suggest that the physicist has no physical images in mind when he speaks, as invariably he does, of spin, interference, resonance, scattering, shell-structure, liquid-drop model, strong and weak interactions, cross-sections, waves, particles, and so forth? To be sure, the quantum physicist can no longer suppose that in the microcosm it is any simple matter of wave or particle. But, taking care only not to mix the metaphors, he productively conceives microcosmic entities now in one way, now in the other, as the occasion demands. There is then overwhelming evidence for the conclusion Schrödinger puts shortly:

Most physicists, whether or no they confess to it, are using some kind of model-picture . . .

"Confession" may be withheld for three reasons. (1) The user of an implicit hierarchic model may easily fail to recognize it as such. (2) He may not want to recognize his use of a model: positivist criticism holds models unsophisticated-and who would not be sophisticated? (3) Even if one would confess, often he cannot. Modern editorial policy of scientific journals suppresses accounts of the course of scientific discovery-in favor of highly compressed, strictly analytic accounts of the nature of the discovery. The published article is then often a complete inversion of the actual process of discovery, likely to omit all mention of heuristically powerful models and analogies that are retrospectively superfluous. Points (2) and (3) together are self-reinforcing: the fewer the references to models in published papers, the greater will be the feeling that their use is somehow disreputable. But even though explicit confession may thus be inhibited, the quantum physicist's vocabulary makes confession for him-and rather strongly suggests that the heuristic power of 
quantum mechanics does not derive primarily from its formal structure.

I am far from arguing that no important discoveries have been obtained from highly formal theories. Consider, for example, the enormous suggestiveness of the purely formal analogy, between physical optics and classical dynamics, to which Hamilton called attention. Moreover, just as a physical model leads us to construe one field in the terms of another, the transfer of a mathematical formalism may perform much the same function. Thus Maxwell remarks (not very charitably) that Mossotti obtained the theory of electrostatic induction, from Poisson's mathematical theory of magnetic induction, simply by translating the magnetic language into electric and the French into Italian. Even highly formal theories that dispense with the "superfluity" of explanation, in terms of overt physical models or analogies, can then function as heuristic devices. I maintain only that they do not function as well as theories that accept some such superfluity. Bridgman, who shares Duhem's point of view, argues:

. . . the mathematical model is just as good as the physical model if it only enables us to answer any question that we may propose about the behavior of the physical system, nevertheless we have an uncomfortable feeling that we have lost something.

I think that we discover on analysis that it is the explanation which we feel we have lost. . . . the mathematical model gives up the possibility of explanation in the usual sense.

The mathematical model cannot be "just as good": to the extent that we give up "explanation in the usual sense" our theory must become a less productive source of questions "we may propose about the behavior of the physical system."

Heuristic power from "superfluous explanation." The physical model or analogy that makes explanation makes also an instrument of discovery. For it is precisely "by considering extensions of the analogy" that we arrive at the pregnant questions which, Hesse says, "suggest extensions of the theory." Toulmin cites, as a good example of this possibility, the conception of light as a "something that travels in straight lines." Beyond explaining the manifold phenomena of shadow-casting, this conception suggests new and important questions (e.g., if light is a something that travels, how fast does it travel?). The model gives rise also to questions ultimately recognized 
as delusive (e.g., is the something that travels a wave or a particle?), as well as to questions obviously nonsensical (e.g., does the something put on its rubbers before coming out?). We can, of course, found a science of geometric optics on a much less ambitious premise: In a homogeneous medium an illuminated point and a point-light are joined by a straight line. The formally superfluous explanation is then suppressed, and with it the delusive and nonsensical questions as well. But alas, the valuable first question, and others like it, are also suppressed: all these questions are suggested by the same explanation.

Torricelli's model is "superfluous." For the correlation of his data a less speculative, more purely descriptive premise would have sufficed: e.g., bodies behave as though subject to a force approximating one ton per square foot of surface in contact with air. Torricelli's seaof-the-air analogy was then formally superfluous, a "pointless" effort to explain the origin of the premised force. ${ }^{*}$ But the heuristic power of Torricelli's concept derives entirely from the selfsame analogy. Only through that analogy does Pascal come to conceive the Puy de Dôme experiment. If we live at the bottom of a sea of the air, ascent through that sea should bring us to regions of progressively diminishing pressure. The experiment leads to genuinely new knowledge: we find a relation between altitude and atmospheric pressure earlier unsuspected. The idea of an atmospheric sea further suggests the possibility of pumping air much as we pump water. Thus efforts to produce a laboratory vacuum derive directly from Torricelli's analogy, and experiments on and in vacua yield a rich harvest of new discoveries. Of course the analogy could also produce misconceptions: taking it too literally, Pascal is led to ask at what altitude one will emerge through a clearly defined "surface" of the atmospheric sea. But the futility of this question is obviously of trivial importance compared to the multitude of worthy questions evoked by the same model-all of which are lost if the model is suppressed.

Basis for the development of aerostatics, the hydrostatic analogue

* Even if only an explanation, may this not still be essential? Without it would Torricelli (or anyone else) have considered for even a moment a premise that demands each of us to assume himself wholly unaware of a total compressive force of some 15 tons exerted on the surface of his body? No small implausibility indeed! Given Torricelli's analogy we at least see some way around the difficulty: after all, fish live and move at substantial depths in water, apparently unaffected by the great pressures to which they are there subject. 
played an important role in other fields as well. In approaching thermal phenomena we may imagine heat a subtle fluid ("caloric") and-passing beyond the analogue-we imagine that fluid self-repellent and essentially weightless. It is through this caloric theory that John Dalton arrives at the set of conceptions that have developed into the modern chemical atomic theory. Moreover, if we conceive "quantity of heat" as measure of the volume of caloric fluid present, and "temperature" as measure of its pressure or hydrostatic head, the hydraulic analogue furnishes us with a suggestive explanation of many simple thermal phenomena. Observe that this analogue and explanation constitute the bridge over which Carnot travels to his celebrated heat theorem, ultimately basis for the second principle of thermodynamics. Note, finally, that electric phenomena (demanding an exactly parallel distinction between charge and potential) are also first attacked successfully through the same analogue-today still commemorated in the term "electric current."

Of course the caloric theory is now rejected in favor of the kinetic theory. It was an explanation that arose "only to be quelled," and perhaps one must concede that this displacement was retarded by the notable plausibility of the explanation. But surely this delay was no disaster. Throughout its lifetime the caloric theory functioned as a highly effective tool of scientific discovery-simply by supplying an explanatory framework in which thermal phenomena become readily conceivable. Ideas of corpuscularity perhaps present the most striking general illustration of the immense heuristic power thus developed. Parmenides made bold to deny the reality of qualitative change, on the ground that he found it essentially inconceivable. Today we laugh at this apt illustration of the follies of rationalism. But observe that it is only by way of Democritus' speculative explanation of qualitative change that we first acquire the power to conceive and work upon it. Duhem, and others of the positivist persuasion, pronounced anathema all atomic hypotheses (and all like speculations about unobservables) and declared vain illusions the explanations provided by them. But those hypotheses have been the prime instruments of an immense scientific progress demonstrably no illusion.

The model taken seriously. Hutten emphasizes that the model must not be taken as a complete "explanation." 
The model . . . is not the same as the thing or process it models; it is analogous to the metaphor. The interpretation by model is partial. But this lack is compensated by the requirement that the model, in fact, must not be a complete interpretation since we want to introduce, with its help, new concepts and construct a higher theory. Mechanical models cannot, and must not, completely interpret, e.g., electromagnetism, since we need room for new concepts, like charge.

We must not then take a theoretic model too literally; indeed, we may err by taking the model too literally. But, as we would realize the full heuristic power inherent in it, we must take the model very seriously. Pascal's idea of a distinct upper surface to the atmosphere is a misconception born of taking Torricelli's model too literally, but the Puy de Dôme experiment is conceived only as that model is taken in dead earnest.

Our models may lead us far astray: we cannot always distinguish "amply seriously" from "too literally." Observing, with Duhem, that these explanatory "superfluities" are often the least enduring features of our theories, we may be led to adopt the positivists' cynical appraisal of physical models and analogies. That attitude denies us the full usefulness of these heuristic devices. If our models are to lead us to ask, and seek answers for, new questions about the world, we must regard them as something more than "logical superfluities," "illicit attempts at explanation," "convenient fictions," or the like. The lesson of scientific history is unmistakable. To the hypothetical entities sketched by our theories we must venture at least provisional grants of ontologic status. Major discoveries are made when invisible atoms, electrons, nuclei, viruses, vitamins, hormones, genes are regarded as existing. De Santillana comments that:

When Leverrier found Neptune "at the tip of his pen," as panegyrics said, he was not simply looking for an economic regularizing entityhe was thinking of a new planet.

Polanyi strongly underlines the point with a citation of a more recent example:

One of the greatest and most surprising discoveries of our own age, that of the diffraction of X-rays by crystals (in 1912) was made by a mathematician, Max von Laue, by the sheer power of believing more concretely than anyone else in the accepted theory of crystals and $\mathrm{X}$-rays. 
No doubt the positivists' is an ideal science-in the sense that "ideal" means the fanciful as contrasted with the actual. No scientific theory has ever been re-constructed, much less created, in a rigorously formal way; no useful report of observables has ever been made free of all burden of inference; no fully productive scientist has ever renounced all hope for explanation, or foresworn all speculation about the true nature of a "real world" unobservable as such. Approach to the ideal-insomuch as necessary, insofar as possible-has throughout the modern era been a routine part of the professional common sense of scientists who have, however, been inspired and guided by concerns and concepts positivism would outlaw.

Bent on discovering the system of the real world, Copernicus did not need positivism to teach him the wisdom of freeing observables from their customary freight of inference. Wholeheartedly accepting the metaphysical assumption of a cosmos mathematically harmonious, Kepler did not require the tutelage of positivism to see the significance of an apparently trivial discrepancy between theory and observable. Not Galileo but Galileo's scholastic opponents preach the supremacy of observables; and all this devout soul's trouble with his church grew out of his stubborn refusal to accept what was urged on him by friend and foe alike: the positivist view of scientific theory as no more than economic description. Newton had not to be taught the virtue of formal construction (virtue already amply apparent to Euclid and Archimedes) when-his thought suffused by a metaphysical corpuscularianism required, for example, to give meaning to his concept of mass-he made a universe with his concept of a gravitational force. These heroes of scientific history did not become so despite the fact they were not positivists: they could become so only because they were not positivists.

The manifold displays of the heuristic power of atomism are founded on the willingness of scientists to suppose atoms real. William Wollaston, the highly gifted contemporary of John Dalton, had a "properly" sceptical opinion of the status of the Daltonian atom. Informed by the same strain of thought soon to produce positivism, Wollaston proposed completely to bypass the difficult problem of assigning relative weights to purely hypothetical atoms.

. . . since the decision of these questions is purely theoretical, and by no means necessary to the formation of a table [of equivalent weights] adapted to most practical purposes, I have not been de- 
sirous of warping my numbers according to an atomic theory, but have endeavored to make practical convenience my sole guide, . . .

Renouncing all speculation about "purely theoretical" entities, Wollaston sought only an economic description of observables. And, indeed, if Wollaston is today at all remembered as a chemist, it is as the inventor of the chemical slide rule: a practically useful calculational device, and nothing more. Compare the meagre harvest achieved by Wollaston's caution with the abundant fruits of the daring of Dalton, Avogadro, Berzelius, and a host of others. Surely one can generalize to all fields of science the conclusion Born puts thus:

All great discoveries in experimental physics have been due to the intuition of men who made free use of models, which were for them not products of the imagination, but representatives of real things. 


\section{CHAPTER IX}

\section{The Evolution \\ of Scientific Theories}

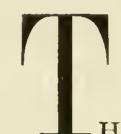

HE MOST impressive features on the skyline of science are its great theories. "Science has its cathedrals," say Lewis and Randall, "built by the efforts of a few architects and of many workers." But those cathedrals prove sadly mutable. Most of the oldest structures have fallen into decay. Even much newer edifices have not remained intact; and some have been moved bodily to new foundations, where they become wings of still greater structures. The continuous turmoil of construction and reconstruction may well repel the nonscientific onlooker. Wishing to see theoretic construction rendered enduring, he would hope to see theories subjected to definitive and final proof. But this possibility simply does not exist.

Consider the logical constellation. A scientific theory is constituted, we saw, by the affiliation of a model $(M)$ and a formalism $(F)$, only one of which may be wholly explicit. Provisionally accepting some theory $(M F)$, I derive from it a multitude of colligative relations, as shown in this schema:

$$
\text { If }(M F) \text {, then } A\left(x, a_{x}\right), B\left(y, b_{y}\right), C\left(z, c_{z}\right), \ldots
$$

I represent a typical colligative relation as $A\left(x, a_{x}\right)$ to indicate that, given specification of certain initial and/or boundary conditions, $x$, the relation $A$ permits prediction of one or more observable condi- 
tions, $a_{x}{ }^{*}$ Undertaking a series of trials with different conditions represented as $x=1, x=2, x=3, \ldots y=1, y=2, \ldots$ and so on, I then predict that I will obtain certain sets of results if each of the relations is sound.

$$
\begin{aligned}
& \text { If } A\left(x, a_{x}\right) \text {, then } a_{1}, a_{2}, a_{3}, \ldots \\
& \text { If } B\left(y, b_{y}\right) \text {, then } b_{1}, b_{2}, \ldots \\
& \text { If } C\left(z, c_{z}\right) \text {, then } c_{1}, \ldots
\end{aligned}
$$

Hence I predict that in certain circumstances some particular observable results will be obtained if the theory is sound.

$$
\begin{aligned}
& \text { If }(M F) \text {, then } a_{1}, a_{2}, a_{3}, \ldots \\
& b_{1}, b_{2}, \ldots \\
& c_{1}, \ldots
\end{aligned}
$$

This is an amply general schema and, as we will see, also a somewhat oversimplified one.

Let us suppose that I confirm a great multitude of predictions $a_{1} \ldots$ The theory $(M F)$ is then confirmed, but it is not verified. Each additional prediction borne out adds some measure of evidence for the theory, and the sheer number of successful confirmations may seem to place it beyond any reasonable doubt. But all these measures together fall far short of proof in any logical sense. Indeed, they can show neither the necessity of the theory (i.e., the impossibility of an alternate theory, perhaps not as yet conceived) nor even its universal sufficiency (i.e., the impossibility of contradictory data, not as yet discovered). Thus, toward the end of the 19th century the system of Newtonian mechanics had been exhaustively confirmed by observations of the most diverse sorts. But only a few years later this classical mechanics was held insufficient, and all the observations by which it had earlier seemed "verified" were shown predictable also from Einstein's new relativistic mechanics.

We can no more think of verifying a theory of physics than a doctrine of metaphysics. But, because the experiential relevance of a scientific theory is always guarded, we can take advantage of a strik-

- The variables comprised under $a$ and $x$ are in large degree interchangeable, but this does not affect our present concern. 
ing logical asymmetry. Suppose that among other predictions of theory $(M F)$ one, say $c_{11}$, fails of confirmation. Then, as Popper notes, we have the possibility of making the only logically valid inference that is truly inductive: the syllogism modus tollendo tollens draws a general conclusion from particular instance $(\mathrm{s})$ :

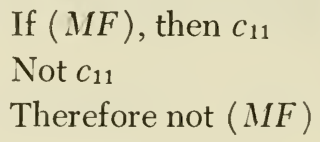

This possibility of falsifying $(M F)$ is of vital importance to a science that progresses through a method of rejection. But falsification, so clear and simple in logical principle, becomes in scientific practice a matter of immense complexity.

\section{THE CRUCIAL EXPERIMENT}

Very large claims are sometimes made for the crucial experiment. Some have even argued that, indirectly, it furnishes us with a method of proof in the positive sense. Given two or more theories exhausting all conceivable possibilities, can we not demonstrate one simply by way of crucial experiment(s) falsifying the other(s)? That question answers itself: the force of the proof depends wholly on the hidden major premise that the theories actually conceived represent all ever conceivable-and this premise is neither proved nor provable. On occasion just two possibilities may well seem to exhaust all alternatives. Light, considered as a something that travels, would appear to admit of only two mutually exclusive interpretations: either light is a wave, or light is a particle. Disqualifying one, do we not unequivocally establish the other? In the 19th century Arago answered this question with a categorical affirmative, and proposed an experimentum crucis (measurement of the relative velocities of light in air and in water) from which he thought one might draw conclusions as follows:

Does the image of the upper point appear at the left of the other image?

Light is a body.

Is the contrary the case? Does the image of the upper point appear at the right?

Light is an undulation. 
Performing the experiment, Foucault found the image on the right; and he, and practically all his contemporaries, considered it proved that light is a wave and not a particle. Today, however, a new species of particle theory is very much alive. We credit a third possibility unknown to Arago: both wave and particle concepts may be required to render full account of the phenomena of light. Failing to supply proof, this excellently conceived experiment thus failed to supply even a final disproof. The whole conception of "the crucial experiment" is indeed subject to major complications, of which we now examine three.

Situations of deadlock. Within the always finite range of experimental error, two theories may be equally concordant with all accessible facts. Imagine, for example, that a theory of special relativity had been proposed long before the discovery of nuclear phenomena and the invention of devices for producing high voltages. Now according to classical mechanics a particle with rest mass $(m)$ and charge $(e)$ which has fallen through a potential drop $(V)$ will be expected to have a velocity $(v)$ given by the equation:

$$
e V=\frac{1}{2} m v^{2}
$$

In relativistic mechanics the equation becomes:

$$
e V=\frac{1}{2} m v^{2} \cdot \frac{1}{\sqrt{1-\left(v^{2} / c^{2}\right)}}
$$

The second equation reduces to the first in the limiting case of velocities small in comparison with that of light $(c)-i . e$., when $v<<c$, $1-\left(v^{2} / c^{2}\right) \doteq 1$. And so, until extremely high velocity bodies are obtained, no crucial experiment will distinguish between these formulas-or between the many other such paired formulas, equally concordant within experimental error, derivable from the two theories. If this is everywhere the case, no experiment can break the deadlock between the two. One may perhaps object that this quite artificial example never actually occurred. But consider that, even with the immense experimental resources of 20th-century physics, after the passage of half a century, we are still unable to devise an experiment sensitive enough to discriminate between the general relativity theories proposed by Einstein and by others.

Such conditions of deadlock occur even when only purely quali- 
tative data come in question. Pasteur and Tyndall maintain the nonoccurrence of spontaneous generation. Pouchet and Bastian maintain that it does occur. All agree that nutrient media boiled for a long time will remain sterile in contact with sterile air, and that media boiled only a short time may in these conditions develop an abundant microbial life. Pasteur and his cohorts argued that in the second case the medium simply had not been sterilized, as it could have been by longer boiling. Pouchet and his partisans argued that in the first case the "vegetative force" had been destroyed by long (or repeated) boiling, so that the medium was no longer capable of engendering life. No experiment then performable could resolve this issue, for Pasteur could no more prove the presence of spores in the second case than Bastian could prove the presence of a vegetative force in the first.

During the first half of the 20th century we find an analogous protracted dispute over whether the crystalline viruses are or are not "alive." No crucial test was feasible simply because these viruses could be grown only in culture media containing living cells. One could then equally well maintain either that viruses are alive but require this special culture medium or, alternatively, dead but capable of commandeering the metabolic machinery of living cells for the proliferation of virus material. Once again there is no dispute about facts, perhaps resolvable by experiment, but dispute only about interpretations of facts, not readily so resolvable. Here, as in many other such cases, the dispute is ultimately resolved through the dissipation of a once-sharp dichotomy. Still other cases may ultimately be settled with the contrivance of more searching experiments. But what may happen ultimately is completely irrelevant to the fact that in situations of deadlock no crucial experiment can help us reach a decision now.

The ramified chain of reasoning. When well accredited ideas are challenged by the result of a purportedly "crucial" experiment, our first impulse is always to question the fact as such. May it reflect no more than illusion or hallucination, as displayed in reports of flying saucers? May it reflect human error-observational error in reading the scales of a telescope, or calculational error in computing the orbit of some satellite? May it reflect some kind of instrumental error -defective graduation of the telescope scales, or some uneven settlement of the foundation for that telescope? Or may it reflect some 
failure in the design or conduct of the experiment, such as improper preparation of the experimental subject, inadequate control of experimental conditions, or instability of the observational devices? Or may the error actually lie in some earlier, related determination the experimenter has taken for granted? Despite the immense number of such possibilities, confirmatory investigations usually permit reasonably prompt dismissal or substantiation of the challenging fact. Suppose that fact is established as a fact: Conant's reminder then comes aptly.

. . . few if any hypotheses on a grand scale or conceptual schemes can be directly tested.

A whole chain of reasoning connects a conceptual scheme with the experimental test.

Defending the theory against the challenge of the fact, we may begin by re-examining the linkage of the fact with the particular colligative relation it calls in question. Have appropriate corrections been made for all the complicating factors that (though unmentioned by the relation) may affect the observation, e.g., for the effect of atmospheric refraction on the observed positions of some body beyond the atmosphere? When we make such corrections we "refine" the denotations attached to our concepts, but we may also think to overhaul these denotations more drastically. Will the supposed equivalence of any alternate denotations involved bear re-examination? Can the denotations be so amended that the relation $C$ furnishes an amended prediction $c_{11}$ ' concordant with what has been observed, or even so that the relation can no longer be applied in situation 11? In such amendments we have substantial freedom but not complete license: we must not impair the previously confirmed predictions drawn from $C$ and other relations involving the same concepts. We may indeed find no acceptable amendment.

We will pass on then to reconsider the relation $C$ itself. Is $C$ less generally applicable than we have supposed? Does it perhaps apply only in certain "ideal" conditions of which 11 is not one? Is $C$ perhaps only the limiting form of a more complex law $C^{\prime}$ which, within experimental error, yields elsewhere the same predictions as $C$, but in situation 11 the prediction $c_{11}$ '? As long as predictions drawn from $C$ were well confirmed we will have been little disposed to question its derivation from theory $(M F)$. But now we may well ask: have 
the syntactic rules been properly applied? Does the derivation involve approximations or assumptions, failing in the (nonideal) situation 11 , without which we actually attain the relation $C^{\prime}$ ? Again we have manifold opportunities for remedial action, but again those opportunities are bounded: we can permit ourselves no action that impairs the theory's capacity to construe the many other relations it has earlier handled in convincing fashion.

And so at last we may be driven to critical re-examination of the theory itself. Ordinarily we will find it difficult to alter the fundamental axioms, to deal with situation 11, without producing new complications elsewhere in the theoretical configuration. However we can always save the theory by adding to its postulates one or more ad hoc assumptions expressly designed to substitute $c_{11}$ for $c_{11}$. With such additions we construct a new theory $(M F)^{\prime}$ which contains the complete essence of the old. Thus the core of the Ptolemaic system-a stationary earth and celestial motions compounded from circles-was maintained by the addition of further epicycles, equants, and eccentrics. Nor is it only fundamentally unsound theories that require such salvage operations. Note that Copernicus' own system deploys some thirty-odd epicycles. For benzene Kekulé is forced to postulate bonds with a wholly unprecedented degree of lability, in order thereby to reconcile his valence theory with the observed isomerism of substituted benzenes. In our own era the neutrino is called into being to maintain the conservation of energy in beta decay.

Given such distinguished precedents, we may then well elect to bolster a prepossessing theory with one or several "saving graces." This possibility is always open to us; and beyond it looms yet another, entirely new, line of defense. Without making any alteration whatever in the theory we can perfectly reconcile it with the challenging fact! For of course the ramified chain of reasoning which links fact with theory involves not just one theory but, as Weyl notes, a whole galaxy of other (potentially alterable) theories and relations.

Individual scientific statements cannot be ascribed an intuitively verifiable meaning, but truth forms a system that can be tested only in its entirety.

The totality of what is tested. Pascal considered the Puy de Dôme experiment a decisive disproof of the theory that Nature abhors a 
vacuum. Ascending the peak, Perier had found a progressive diminution in the height of a barometric column of mercury. This diminution Pascal held to signalize a decline of pressure, with increasing altitude, wholly inexplicable by the older theory. For, said he, one can hardly maintain that Nature abhors a vacuum any less at a mountain's top than at its base. But any who might have wished to maintain the theory of horror vacui were not driven to any such "absurd" contention. They could perfectly well have held the central theory intact by challenging the ancillary assumptions involved in the test. For example, Pascal simply assumed that the density of mercury is much the same at mountain top and base. However plausible, the assumption was not checked (and might have failed if, for example, mercury contracted rather more than it does with decreasing temperature). Pascal assumed that the ratio of length of a column of mercury and a wooden measuring stick remain unaltered with changes in their position, which is amply plausible but unconfirmed. He assumed further that bodies are drawn to earth no more strongly on mountain top than at mountain base, and so on. Had an attempt been made to check such assumptions as these, still other assumptions like in kind would have been involved in the checks, and so on ad infinitum.

When experimental finding conflicts with theoretic prediction, we learn that something is amiss in the complex conceptual structure used to produce that prediction. But, as Duhem long ago emphasized, the exact locus of error is just what the experiment does not teach us. The logician Quine makes the same point with an excellent metaphor.

. . . total science is like a field of force whose boundary conditions are experience. A conflict with experience at the periphery occasions readjustments in the interior of the field. Truth values have to be redistributed over some of our statements. Reëvaluation of some statements entails reëvaluation of others, because of their logical interconnections- . . But the total field is so underdetermined by its boundary conditions, experience, that there is much latitude of choice as to what statements to reëvaluate in the light of any single contrary experience. . . .

Any statement can be held true come what may, if we make drastic enough adjustments elsewhere in the system.

Clearly this extreme statement fails entirely to render due account 
of that hierarchy of theoretic propositions noted in Chapter VII. For, indeed, if nothing could be held comparatively well established, science would promptly collapse into chaos. When some wild idea of mine has failed an experimental test, certainly I am not disposed to argue (nor my colleagues to hear the argument) that my idea is perfectly sound, and that what has actually failed is one or more of the substantive principles necessarily assumed in making the test. Thus Feigl correctly stresses that some ("established") propositions will and must always be maintained far more tenaciously than other ("hypothetical") propositions.

A view that maintains that the whole body of a scientific theory (if not of all science) confronts experience and that modifications may be required in any part of the system if it does not "fit,"-such a view obscures dangerously what is of the greatest importance for the progress of science: the successive testing and securing of parts of science -at least in the sense of an approximation. Naturally, no part can be considered as established with finality-but this insight which impresses the pure logician should not blind him to the recognition of the method of successive confirmation.

Wholly inadequate as an account of ordinary scientific practice, Quine's statement correctly depicts the situation in logical principle. And on rare occasions some such situation is found in practice. Very seldom do we propose to jettison a well-founded relation to save one less well-established-though Marignac was apparently quite prepared so to jettison the proposition that masses are additive, thereby to preserve Prout's highly speculative hypothesis in the face of contradictory data. But not uncommonly we will propose to jettison one relation in order to preserve another not much less firmly foundedas when Mendeleev seeks to preserve his periodic classification by questioning the general applicability of Dulong and Petit's law, which indicated for beryllium an atomic weight incompatible with that classification. And always, finding it almost literally unthinkable that there is some fundamental unsoundness in a major theory to which we can conceive no alternative whatever, we will jettison practically anything and everything to preserve that theory. Thus, to save classical electrodynamics in the face of the result of the Michelson-Morley experiment, Fitzgerald proposes that the length of, say, a meter stick is not constant but systematically variable with its velocity. 
The crucial experiment in practice. The possibility of falsification, so simple and definitive in principle, seems to have dissolved into hopelessly complex equivocality as a matter of scientific practice. Yet the crucial experiment is not wholly a myth: on some historical occasions one or a very few observations or experiments have been widely accepted as rendering a decisive verdict. This is just the dramatic effect we expect of that species of crucial experiment the logical impossibility of which Duhem and Quine emphasize. How then is any such effect produced?

An experiment may function as crucial simply because its result is, quantitatively and/or qualitatively, so completely out of line with anything foreseen by the upholders of one theory, though perhaps predictable on another. After the fact that first theory might be "saved" by any of the many expedients we have indicated. But the brute blow of the totally unexpected can stun resistance and paralyze any such rescue operation. The crucial effect derives then not from logical force but from psychological impact.

Consider too the effect of the sociological context. All the participants in a modern scientific dispute generally agree that a very great deal is to be taken as of principle, insusceptible to change. Hence, though many other relations and theories are involved in the test of some one disputed proposition, even those on the losing side of a crucial experiment may not seek to deny its force by impugning other parts of scientific knowledge. Arago's proposed experiment was made crucial when all concerned, in effect, agreed in advance simply to rule out of court a great variety of expedient assumptions that, after the fact, might yet have been used to "save" whichever theory the experiment discredited. In part producer of the unity of organized science, the crucial experiment is thus also in part product of that unity.

Beyond the logical, the psychological, and the sociological contexts, we come at last to the most important of all: the scientific context. We can no more suppose a major development in science the effect of a single crucial experiment than we can suppose World War I the result solely of the assassination of the Archduke Ferdinand. A successful scientific theory renders indispensable services in the correlation and explanation of what we already know and, as a heuristic device, in supporting our search for new knowledge. Until we find some alternative theory that promises to perform these services 
as well or better, we will stubbornly defend the threatened theory. Having observed the inexhaustible resources that can be brought to the defense of any given theory, we see that no crucial experiment can force on us a final absolute falsification of that theory. But what such an experiment may well do is to sway our judgment to a verdict that, compared with another, the given theory is relatively less sufficient. That is, ultimately we accept falsification of the theory when we consider it has been shown functionally inferior to a competitor.

\section{Three Functional Criteria}

All possibility of strictly logical verification or falsification being denied us, we must in the end look to the functional criteria. Complex, uncertain, in a degree irreducibly subjective, they are at least usable criteria.

\section{CORRELATIVE EFFICIENCY}

Correlative efficiency seems the most straightforward of the criteria by which we might appraise a scientific theory. We might for example conceive a correlative index thus:

$$
\begin{aligned}
& \begin{array}{c}
\text { Number of } \\
\text { independent colligative relations } \\
\text { accommodated in theory }
\end{array} \\
& \begin{array}{c}
\text { Number of } \\
\text { independent postulates required } \\
\text { to constitute the theory }
\end{array}
\end{aligned}
$$

Can we not always choose with confidence, among competing theories, that one with the highest value of the index ratio? But which does offer the better index ratio: Avogadro's theory, which uses more postulates to correlate more relations; or Berzelius' theory, which uses fewer (and less extreme) postulates to correlate fewer relations? One might then wish for quite precise evaluations of the index ratios, and I do not believe any such evaluations possible.

Can we even estimate the numerator for any major theory? And were we given some such estimate of number, could it mean anything if we did not attach to each relation some factor indicative of 
the tightness of its accommodation in the theory? Scholastic science accommodated practically everything known, but in a system of extreme looseness. Such looseness is reflected in the production of the relations through deductive chains flawed by assumptions and approximations for which the theory provides no explicit justification. But this looseness was wholly unapparent (as almost invariably it is) to contemporary eyes. Perhaps we may hope to estimate tightness of correlation by considering the extent to which the theoretic derivation rationalizes the limits in range and accuracy we find in practice to attach to each colligative relation. We may hopefully adopt a modified index ratio of the form:

$$
\frac{\Sigma R_{i} t_{i}}{\Sigma P}
$$

Here $t$ is the factor of tightness ranging from 0 , for a relation entirely unaccommodated, up to 1 for a relation that has both its strengths and weaknesses perfectly delimited.

Turning now to the denominator, we face much graver problems. Dealing, as always we do, with incompletely formalized theories, we never have a definite roster of postulated axioms, syntactic rules, semantic rules, auxiliary assumptions, etc. Using common sense and our knowledge of other sciences, as indicated in the preceding chapter, often we simply rely on intuition to supply the necessary items, as we need them, without any full awareness of their identity, much less their total number. Even given such a number, we would have yet to render account of the fact that sometimes a simplification of syntactic rules demands an entirely disproportionate complication of semantic rules, and conversely. Attach to each theoretical stipulation some factor of complexity, f? If possible, why not? We have then to face "only" this terminal problem: can we even hope to state a correlative index for any single theory when, as we have just seen, in every application it forms a unit with unnumbered others of its kind?

Noting these complications, and however improbably, let it be granted that a correlative index can somehow be estimated. Often theories will seem to differ so sharply in index value that even crude estimates are sufficient to make the difference manifest. Do we then have a criterion sufficient for the judgment of competing theories? Plainly it cannot always be sufficient. Whatever may be the ultimate 
judgment of its correlative efficiency, a brand new theory often fails to shine in this respect.

The insufficiency of the correlative index. Ordinarily the creator of a new theory has conceived a new interpretation for some particular data not smoothly accommodated in an older system of thought. Often he can see in outline how to extend his conceptions to a much larger body of data, but sometimes he cannot at once demonstrate the applicability of his ideas even to all that the older system took in. He will then exclude from consideration certain whole groups of phenomena. He is the more disposed to this policy because usually, between his theory and at least some of the excluded data, there will be discrepancies he would have somehow to explain away were not the whole group of phenomena excluded. As it is, he will almost certainly have encountered some discrepancies between his theory and a few of even the relatively small central group of data on which the theory turns. These discrepancies he must deal with, and most often he does so by adding to his theory various ad hoc postulates-if only such as argue the inaccuracy of the conflicting data. The supplementary postulates, taken together with a very restricted scope of application, may then burden the new theory with a correlative index distinctly inferior to that of the established theory it would displace.

Though it represented the labor of a lifetime, Copernicus' system displays no clear superiority in correlative index. It drops entirely out of correlation all the physics accommodated with the Ptolemaic system as part of the scholastic synthesis; and, if it requires somewhat fewer epicycles, it requires rather more numerous ad hoc postulates to explain away the apparent fixity of the earth, the absence of detectable parallax, etc. Consider as a second example Lavoisier's new chemical system. Where the phlogiston theory had postulated few elements but multiple states or conditions of phlogistication, Lavoisier postulates many elements. Let us suppose that initially the numbers of postulates were roughly equal. We will then judge Lavoisier's theory superior for its convincingly tight correlation of the phenomena of oxidation and reduction. But we must also observe that at its first appearance this theory accommodated practically nothing else and, even within the focal domain, Lavoisier concedes his theory's incapacity to cope with Priestley's "stubborn facts." On the other hand, the phlogiston theory took in (albeit loosely) a very large part of chemistry-including appearances of colors and thermal effects 
that go entirely unmentioned by Lavoisier's original theory, though they are perhaps the most striking manifestations of its focal phenomena. Consider finally the interpretation of the photoelectric effect proposed by Einstein. This interpretation excluded practically everything but that one complex of effects. Yet it introduced a major element of discontinuity gravely out of keeping with the theory of classical electrodynamics by which an enormous number of relations were accommodated. The correlative index of Einstein's theory might then be held contemptible; and his work was indeed harshly criticized by Planck, much as Copernicus' was criticized by Bacon, as an irresponsible innovation that leaves entirely out of account much more than it takes in.

These examples amply suggest that, if certain new theories are ever first to be considered, the correlative index must not figure as an immediately decisive criterion of judgment. A number of quite different examples suggest that in fact it is not so regarded. Newton unites astronomy and terrestrial physics in a single system with correlative index approached by no other theory of his time. But some of Newton's not least acute contemporaries rejected his theory. Darwin proposes a theory with correlative index of a very high order, at first rejected by many if not most contemporary biologists. Such rejections are more probable when, as in the cited cases, the new theory seems to have cosmologic overtones; but they occur even when no such overtones are present. Arrhenius' theory of ionization handsomely joined the phenomena of electrochemistry with a multitude of other phenomena of chemical reactivity, color, osmotic pressure, vapor pressure, etc. It represented a major advance in correlation, and it too was quite widely rejected on its first appearance.

\section{EXPLANATORY APPEAL}

Presumably what is for us explained is only what we find derivable in some system of correlation. If so, there can be no explanation save by way of correlation, but this does not at all establish that explanation is nothing but correlation. Indeed, when men reject new theories that represent substantial advances in correlative efficiency, they generally do so because they find inadequate the explanatory appeal of those theories. Clearly then something more than correlative efficiency must enter into the making of explanatory appeal.

A theory's explanatory appeal usually depends primarily on the 
plausibility of its analogy or model. Copernicus' theory proposes to explain the diurnal motion of the heavens using a model that represents man facing the heavens as one who in a moving ship observes the "motion" of the shore. Owing to our unawareness of the motion of our "ship" this model seems much less plausible than Ptolemy's, in which the heavens move as does a ship seen by a stationary observer on the shore. Lavoisier's new theory is at first criticized for the sheer implausibility of its postulation of a host of elements when "everybody knew" that there could be but one or a few fundamental elements. Newton's theory is rejected by Leibniz for the sheer absurdity of what he took to be Newton's idea of a gravitational force acting across the abyss of interplanetary space. The "obvious" difference between thinking man and dull beast gave an air of implausibility to Darwin's conception of evolution, and the marvelous organization of living things to his concept of a random "natural selection." Who could seriously credit Arrhenius' view that oppositely charged ions are "spontaneously" separated from each other in solutions that draw on no outside source of energy?

Quite obviously explanatory appeal is a complex function of a multitude of scientific, cosmologic, and cultural factors. Observing the profoundly subjective quality of such appeal, we may, if we like, deplore its use as a criterion of scientific judgment. Yet, as we saw earlier, the appeal of its mathematical harmony was very nearly the sole support of the Copernican theory in its youth. In any case, whether or not the criterion of appeal should be disregarded, quite plainly it has not been disregarded. Scientific theories are humanly judged by men never inhumanly unmindful of explanatory appeal and the subjective considerations that determine it. To the extent that judgment is affected by such appeal, we find here a major bias toward conservatism. An established theory learned early in life will generally have for us a plausibility no genuinely novel theory can later rival.

The two criteria of immediate judgment. Efficiency and appeal are the only criteria always available for judgment of a totally new theory. Ordinarily some time must pass before we can gauge its heuristic power by the full range of discoveries to which it may or may not lead. And have we not then reached a complete impasse? The criterion of efficiency is often biased against novelty, the criterion of appeal almost invariably so. Must we not then conclude that a funda- 
mentally new theory will, on first presentation, almost always be flatly rejected? However, this is no reductio ad absurdum but, rather, just what Taton observes to be historically the case.

Every great discovery has produced some sort of intellectual scandal, has been opposed by current, and always badly informed, opinions on the basic nature of scientific problems, and also by the majority of scientists of the time holding outdated theories, and incapable of renouncing some of their most solidly ingrained ideas.

The first two criteria taken singly, or together in appraisals of "simplicity," are then insufficient to ensure sound judgment of new theories. Presumably we must pin our hope for such judgment to the third criterion: heuristic power. Yet the first two criteria are not then inconsequential. Before it can display its heuristic power, a new theory must win at least provisional accreditation by men willing to accept its guidance in their work. Such accreditation can derive only from the sense that the new theory offers some promise, if not yet any realization, of superior correlative efficiency and/or explanatory appeal. Consider too that, as earlier noted, both the correlative and explanatory functions enter into the heuristic function. We saw in the preceding chapter how prominently the explanation offered by a theory figures in its use as an instrument of discovery. And of course the theoretic correlation permits us effectively to marshal the knowledge we already have in the quest for new knowledge. Finally, as we will see, the heuristic functioning of a theory may so alter the balance of efficiency and appeal that in the long term "simplicity" acquires a large significance it lacks in the short.

\section{HEURISTIC POWER}

Duhem minimizes the explanatory function of a scientific theory, stresses its correlative function, and never renders account of its heuristic function. Dirac, too, minimizes the explanatory function, but only to emphasize the supreme importance of the heuristic function.

The only object of theoretical physics is to calculate results that can be compared with experiment, . . .

. The object of quantum mechanics is to extend the domain of questions that can be answered and not to give more detailed answers than can be experimentally verified. 
How strange that a theory should be valued primarily as a source of new questions, rather than as a set of new answers to old questions. Yet no stranger than that the Eskimo values the fish-hook more than the fish that alone sustains his life, or that the pioneer woodsman valued more the flint-and-steel than the fire which alone gave him warmth. The flint-and-steel offers the means of making fires; the hook, the means of catching fish; the scientific theory, the means of winning new knowledge.

A theory is expressly designed to provide accommodation for a certain number of known relations. Its success in doing so we may regard as testimony for its creator's ingenuity rather than for the theory's own intrinsic soundness. After all, in logical principle we accept the possibility that those same relations might be correlated by some quite different theory, and Joseph Black long ago observed that:

A nice adaptation of conditions will make almost any hypothesis agree with the phenomena. This will please the imagination but does not advance our knowledge.

However when, serving as a heuristic guide, a theory does "advance our knowledge," we begin to think of it as more than just "a nice adaptation of conditions." We may so advance in knowledge even of relations already known. Thus, for example, a new theoretic derivation may suggest some large extrapolation of a relation earlier thought limited in applicability or, contrariwise, indicate (by the assumptions and approximations involved) sharp limitations to a relation earlier thought general and exact. Again, suppose we find that the theory can easily be extended to rationalize some relation(s) quite outside its original scope. Thus Whewell observes that when the long-familiar precession of the equinoxes was brought within the explanatory framework of the Newtonian synthesis, originally designed to deal with quite different motions, this "gave to the theory a stamp of truth beyond the power of ingenuity to counterfeit." Finally, who can fail to be impressed when the theory leads to $\mathrm{en}$ tirely new knowledge?

Through Gibbs' phase rule we come at last to see a profound relation hidden in data already long available, but first rendered comprehensible by Gibbs' concept of chemical potential. Consider, too, the striking example presented by Mendeleev's periodic classifica- 
tion. By it chemists are led to seek and find new elements, with properties almost exactly those predicted by Mendeleev. When a theoretical construction thus guides us to major new knowledge, whether of old or new relations, we obviously get out of it something more than the ingenuity of the most perspicacious of creators could have put into it. Have we not then attained conclusive evidence for the fundamental soundness of that construction? And have we not also identified, at last, the critical factor in judgment of scientific theories? The decisive criterion is heuristic power, and some particularly striking display of such power may then be taken, as Pascal took the Puy de Dôme experiment, to be an experimentum crucis. But even yet our story is far from fully told. After all, for many of Pascal's contemporaries his was not a crucial experiment, and the theory of horror vacui long survived Pascal himself.

When we draw from a theory new questions that lead us to important discoveries, we do not thereby establish the validity of the theory. Polanyi notes that some quite erroneous values for the ratio of the atomic weights of hydrogen and oxygen prompted Urey's search for a heavier isotope of hydrogen, for the discovery of which he was awarded a Nobel prize. Of a notable theoretical advance Fierz remarks:

... it may well be that on the basis of apparently wrong and irrational speculations a physicist discovers something that is correct and important. This was the case with Dirac's theory of the electron where the dogmatic postulate (which is also quite wrong) that his equations should be of first order, finally led to the correct theory.

Observe, too, that the discoveries made with a given theory may derive less from its intrinsic virtues than from the superior talent (or energy or facilities) of a man or men who adopt it. Newton accomplished far more with his clumsy geometrized calculus than Leibniz did with his. Observe further that the root of heuristic success may lie not in the explicit content of a theory but in some implicit attitude associated with it: thus it is, I think, that teleology has led to some substantial discoveries. Observe finally that, in De Morgan's terminology, "wrong theories" can be "rightly worked": today we reject the phlogiston theory that led Priestley to discover photosynthesis and Scheele to the first discovery of oxygen. Thus even the gaudiest displays of heuristic power cannot coerce our acceptance of a theory, 
or allay completely the doubts of all who doubt it. And so again we seem to reach an impasse: how can a new theory ever win general acceptance? The answer lies in the deeper secondary effects of its heuristic power.

\section{The Natural Selection of Scientific Theories}

Polanyi observes that

A hostile audience may . . . deliberately refuse to entertain novel conceptions such as those of Freud, Eddington, Rhine or Lysenko, precisely because its members fear that once they have accepted this framework they will be led to conclusions which they-rightly or wrongly-abhor. . . .

We can now see, also, the great difficulty that may arise in the attempt to persuade others to accept a new idea in science. . . . to the extent to which it represents a new way of reasoning, we cannot convince others of it by formal argument, for as long as we argue within their framework, we can never induce them to abandon it. Demonstration must be supplemented, therefore, by forms of persuasion which can induce a conversion.

Particularly when cosmologic issues are involved, no analyses of, or arguments from, the criteria we have examined can constitute such persuasion. No demonstration of mathematical elegance could reconcile some men to the immensity of the universe depicted by the Copernican theory; no demonstration of correlative efficiency could persuade Agassiz to accept a theory of evolution by natural selection; no demonstration of heuristic power could convert Planck and Einstein to belief in a quantum mechanics that denies all but statistical determinism. Planck, indeed, denies the whole possibility of conversion in such cases.

An important scientific innovation rarely makes its way by gradually winning over and converting its opponents: it rarely happens that Saul becomes Paul. What does happen is that its opponents gradually die out and that the growing generation is familiarized with the idea from the beginning: . . .

How does it happen that new opponents are not born to replace those who die? We discern a mechanism of natural selection that 
brings one species of opinion to triumph, in the struggle with its competitors, if only it displays superior heuristic power.

One or a few men grasp a daring new idea. If it is to survive they must at once make it count for something: well has it been remarked that when skating on thin ice one must keep moving. Let us suppose the new idea heuristically powerful enough to lead those who entertain it to a few notable discoveries. These draw the attention of others who aspire to discovery and, adopting the idea at least provisionally, some of these recruits may also profit by it. Those most successful in this way will be favored in the competition for posts of influence, particularly in the universities. Wherever they are or become established, their successes will make them especially attractive to students who also aspire to be discoverers. From the many who compete for the privilege of working with the successful investigator, he will select what seem to him the most promising men of the coming generation. Gaining command of the new theory at the outset of their careers, these students of superior ability can, through their own discoveries, still further enhance the theory's luster.

Meanwhile the die-hard adherents of the old theory make few comparably notable discoveries: the heuristic power of their ideas will by now be largely spent. As time passes such a resistor of conversion may come increasingly to acquire the reputation of a crank -which cannot but still further decrease his capacity to attract able students. And even his students will become familiar with the new theory, through the publications in which its partisans report their unmistakable successes. Given the evidence of those successes, the students may adopt the theory despite their master's opposition. At a slightly later stage the new theory finds its way into textbooks: however conservative the authors of these may be, they can hardly fail to mention a view that provokes important discoveries the texts cannot ignore. And more vigorous statements of the new theory will be given in texts written by its partisañs, whose own successes go far toward ensuring wide use of their books. As the terminology of the new theory thus gains general currency it is even more firmly established-in precisely the manner Joseph Black had in mind when he noted the subversive effect of the new chemical nomenclature Lavoisier and his cohorts had so designed

. . . that the very denominations of the different objects should imply the doctrines of their theories; so that, by using this language, it 
should scarcely be possible to think on chemical subjects in a way different from their theories.

The heuristically potent theory shows itself viable: it proliferates itself in the minds of scientists. After a time its heuristic power may wane, but by then its position is secured. It is enshrined in textbooks, linked in scientific tradition with the names of famous men, woven into the fabric of scientific language and thought. Most important of all, its position is secured because the theory with which it had once to compete has been displaced, crowded out. The once-novel theory is now orthodoxy, maintained (if by nothing else) by sheer intellectual inertia. Natural selection is thus decisive where "persuasion" fails and, within the space of a single generation, a viable scientific theory can so attain practically universal accreditation.

Viability and validity. Natural selection in nature need not select "the good," and natural selection in science need not favor "the true." A slightly deeper exploration of the metaphor will be helpful. In the world of nature, any "part" of an organism reacts with its other parts through an internal environment. Any mutation producing a "part" fundamentally incompatible with the others is then a lethal mutation: those who carry it will not survive. Any surviving mutants then face a further screening, in the external environment with which the organism interacts as a whole, and natural selection will favor just those mutations that give the organism some advantage in its habitat. Thus biological natural selection always functions in terms of two distinct sets of environmental interactions, and all such selection is made relative to two kinds of prevailing conditions. Clearly then the "judgments" of natural selection may well vary with time and situation: once notably successful, the dinosaur is now extinct.

In the world of scientific ideas natural selection also proceeds by way of two sets of interactions-with an inner (conceptual) and an outer (empirical) environment. A new theoretic idea is stillborn, at once rejected even by him who first conceives it, unless it has some minimum degree of compatibility with the intellectual atmosphere of the age. Some still larger degree of compatibility will be required if the new idea is to function effectively. Aristarchus' conception of a heliocentric system was nonviable simply because it was so deeply incompatible with much the ancient world took for granted. The very early (and quite imperfect) anticipations of an oxygen theory, by Mayow and others, similarly failed to "catch on" in a scientific 
world that took it for granted that air is an element. Ideas that do survive such testing by the conceptual environment have then still to show themselves viable in the empirical environment. That is, they must be capable of productive exploitation by the empirical techniques available. In the then-contemporary state of science, ancient atomism probably could not have issued in any works of value. Similarly, though we detect important elements of soundness in Berzelius' idea of electrostatic bonding, it was rejected in the first half of the 19th century because it did not, perhaps could not then, lead to any important new discoveries.

If it is to prove viable, a scientific theory must, in two distinct respects, fit its times. Obviously then ideas that fail at one time may later succeed where formerly they failed (or vice versa). Newton's corpuscular theory of light triumphs in the 18th century; given the phenomena of polarization and diffraction, the undulatory theory triumphs in the 19th; given quantum effects, a new corpuscular view flourishes in the 20th. The times thus ripen toward the dominance of an opinion at one time almost extinct. Large changes in the intellectual climate permit Copernicus to gain for the heliocentric theory a hearing that had been denied Aristarchus; small changes make it possible for Cannizzaro to succeed where Avogadro had failed. Small advances in experimental techniques permit Pasteur to succeed where before him Spallanzani had failed; large advances in observational techniques permit Kepler and Galileo to consolidate the position of the Copernican theory as Copernicus himself could not have.

A notably inadequate theory may enjoy entirely undue viability if (like the Ptolemaic theory) it for long confronts no serious competitor. And two or more competing theories may prove almost equally viable simply because they are all almost equally (in)effectual heuristic guides. We see readily that in such cases-which occur ordinarily only in the earliest stages of development of a science-what fails is the conditions for natural selection, not natural selection as such. But we may think to observe also some genuine failures of that mechanism. When "rightly worked," quite "wrong theories" may be chosen by natural selection-and quite right theories rejected. Apparent miscarriages of this sort may distress us, but should they do so? Conceiving scientific theories as primarily heuristic tools, we may well expect the verdicts of natural selection to be almost invariably 
sound. That is, the theoretic ideas so selected will be generally just those that best seize the opportunities presented, and best support the demands imposed, by the conceptual and empirical situations at a time and place. And these viable ideas are the correct choice, whether or not they prove timelessly valid.

Rejecting the opinion of the alchemists, Lavoisier holds the chemical elements immutable. We find them transmutable. But retrospectively it appears that Lavoisier's was the only conception on which a viable systematic chemistry could be founded in his time. Proust denies, and triumphs over, Berthollet's opinion that genuine compounds are at least slightly variable in their proportions. We now see some merit in Berthollet's opinion. But was not Proust's view absolutely essential for the first conception of a chemical atomic theory that could function in the early 19th century? Similarly, I fail to see how that theory could then have been created save, as it was by Dalton, on the presently-rejected assumption that the chemical elements are uniquely characterized by invariant atomic weights. Newton's concepts of space and time we now question, but for the 18th and 19th centuries the selection of those concepts was absolutely correct. Our present scepticism of those concepts is indeed very largely the result of developments to which they themselves have led us. In each case we see an earlier judgment of natural selection revised or even reversed. In each case, however, the earlier judgment did single out the most powerful heuristic tool for the time.

\section{THE LIFE CYCLE OF A SCIENTIFIC THEORY}

The broad outline of scientific advance develops from the cyclic rise and fall of scientific theories, each of which determines the general direction and extent of such progress as will be made until its successor is born. Kroeber finds that "The Configurations of Culture Growth" can effectively be conceived in terms of a cycle of creationexploitation-exhaustion of cultural patterns. Science is itself a cultural phenomenon, and the life cycle of scientific theories is not without analogy to the cycle of specialized cultural patterns of which Kroeber writes:

The very selection which at the outset is necessary if a distinctive pattern is to be produced, is almost certain later on to become a limitation. . . . It seems to be historically almost as difficult to reconstitute a pattern fundamentally, or to widen greatly the scope of a growth, as 
at an earlier stage it is difficult to get a distinctive pattern growth or pattern value started. Not infrequently, when a pattern has attained realization or reached saturation, its limitations appear to be felt and efforts are made to alter or enlarge it. If these efforts take the form of a pause in activity, there may be a reconstitution of energy and direction, ... growth is renewed along somewhat new and broader lines.

Infancy and youth. As first born, a scientific theory is most often comparatively crude in construction. Taton remarks:

The innovator who reverses a theory and tries to replace it by another cannot hope to produce the most unimpeachable arguments and the most convincing demonstrations. The effort to rebuild an entire edifice, patiently constructed and consolidated by the work of many generations of scientists and by long tradition, is so immense that it is rare for one man to accomplish this transformation definitely by himself.

The new theory will reflect its creator's novel insights, but also his limitations-in part produced by his inability wholly to free himself of the older pattern of thought in which he was reared. The conceptual structure he creates will ordinarily be blemished by substantial inconsistencies, irrelevancies, superfluities, exclusions, or the like.

Advances in observation and experiment may help to bring about improvement, but the major refinements of the new theoretic idiom will be made by eliminating conceptual flaws its creator may have been wholly unable to recognize, much less to repair. Thus Kepler's reconstruction of the Copernican theory-in which all epicycles are eliminated through the introduction of elliptic orbits-demanded a freedom from obsession with perfect circular motion that Copernicus himself probably could not have approved, and never attained. Newton's Principia offers his system in a highly developed form, but an error in the calculation of the lunar orbit remains to be corrected by Clairaut; and approximately a century elapses before Laplace gives Newton's system its definitive and most powerful development.

Dalton's own conception of the chemical atomic theory was so fundamentally defective that he completely failed to realize the immense value to that theory of the discoveries of Gay-Lussac and the suggestions of Avogadro. An adequate foundation for the theory was not constructed until 50 years after Dalton's work, and today we en- 
tertain the theory in a form that Dalton would have found unacceptable. From Faraday's distinctly physical concept of lines of force, Maxwell extracts the sophisticated abstraction of the electromagnetic field; and Maxwell's theory, like Newton's, is given its ultimate expression by French formalists. Bohr's quantum theory is fundamentally syncretic (the central derivation applies classical laws to a markedly nonclassical system) and it involves multiple auxiliary postulates of quantum numbers, etc. The theory is improved through the work of Sommerfeld and others, and finally achieves its definitive formulation in the quite differently slanted theories of quantum mechanics. These theories at last offer fully self-consistent treatments of nonclassical systems, and all the quantum numbers now appear as necessary consequences of the more powerful sets of postulates. But even these theories of quantum mechanics did not spring to being in full perfection: for example, we now entirely reject Schrödinger's original interpretation of the wave function.

The magnitude and time scale of such refinements are extremely variable: their net effect seems generally the same. The theory is completed and perfected. Deploying now an absolutely minimal set of postulates, it is recast in a form of absolutely maximal power and generality. Certain exclusions, originally unavoidable, may now be lifted. From the narrowness and crudity of its weak youth, the theory grows toward the broad and subtle power of maturity. The phase of consolidation shades into the phase of exploitation.

Maturity. Where pioneers once trod hastily and at their peril, the husbandman now tills the soil. The once-daring theoretic ideas are now used routinely, and they impose on scientific research some particular characteristic orientation(s). Thus attention will be directed toward certain phenomena, problems, and experiments and, correspondingly, turned away from others that seem unimportant or not susceptible to study. Work thus prosecuted under the guidance of the theory will fully explore its consequences: all the i's will now be dotted, all the t's crossed. And all the time strenuous efforts will be made to stretch the theory, both in depth and in breadth. That is, we demand that the theory (perhaps originally based on quite crude data) show its competence to construe the results of observations and experiments we are forever improving in accuracy. And we demand also that the theoretic pattern be extended into forever-new domains of experience we regard as contiguous to those in which it 
has functioned successfully. Where in the theory's youth we were happy with small displays of capacity, we now "prove" it by demanding the utmost of it. No longer fearing the challenge of "anomalies" instead, as Poincaré observes, we actively seek them.

. . . after the rule is well established, after it is beyond all doubt, the facts in full conformity with it are erelong without interest since they no longer teach us anything new. It is then the exception which becomes important. We cease to seek resemblances; we devote ourselves above all to the differences, and among the differences are chosen first the most accentuated, not only because they are the most striking, but because they will be the most instructive.

Incredible as it may seem, a great theoretic pattern like Newton's long sustains all these stresses with little or no manifestation of strain. By such stressing, in effect we court strain. We find instead an uninterrupted sequence of brilliant successes extending over centuries. Bit by bit we lose sight of the principle of corrigible fallibility: no longer do we suppose that the theory ever can fail. And when the (invariably equivocal) first appearances of strain materialize, as sooner or later they always do, the general reaction is likely to be very much that Polanyi describes:

Just as the eye sees details that are not there if they fit in with the sense of the picture, or overlooks them if they make no sense, so also very little inherent certainty will suffice to secure the highest scientific value to an alleged fact, if only it fits in with a great scientific generalization, while the most stubborn facts will be set aside if there is no place for them in the established framework of science.

Even in the unlikely event that these are conceded puzzling facts, the vast majority of scientists will still remain blithely confident that future developments will certainly resolve all such puzzles-if only in some way(s) not yet imaginable. Ripening into honored old age, the theory now begins imperceptibly to drift toward senescence.

Old age. Strain sometimes develops simply from the accumulation of precisely the kind of data the theory was designed to handle. Thus the astronomical data collected over centuries produce stresses the Ptolemaic system can sustain only by an ever-increasing elaboration of ever-creakier epicyclic machinery. In other cases the strain develops less from accumulation than from refinement of the focal data. Thus Brahe's data subject both the Ptolemaic system and the 
original Copernican system to a strain we recognize as utterly intolerable.

For a time Newtonian celestial dynamics proved superbly competent to handle planetary observations of constantly improving accuracy. Some minor aberrations presently observed in the motion of the outermost planet were viewed with no great alarm for, as Dingle emphasizes:

Most scientific problems are relatively so superficial that anything so drastic as a change in the basic terms of expression would be beyond reason. When Uranus was found to move in a puzzling way, Adams and Leverrier did not begin to reform the foundations of mechanics. Rather than do that, they were prepared to call a new world into existence [the subsequently discovered planet Neptune] to redress the balance of the old, and they did so within the framework of current mechanical theory.

With still further accumulation of still more refined data, a much smaller aberration in the motion of the innermost planet Mercury became utterly inescapable. And, perfectly naturally, one then hypothesized perturbations by an undiscovered inner planet, Vulcana planet perhaps even unobservable because of its closeness to the sun. Retrospectively we see that the behavior of Mercury strained Newtonian dynamics, and signified its insufficiency. But contemporary judgment showed, could have shown, no such awareness. For how can one, at the time, distinguish between the (sound) Neptune hypothesis and the (unsound) Vulcan hypothesis; between evidence that further stretching of the theoretic pattern is needed and evidence that it is already strained? Polanyi finds

. . . no valid heuristic maxim in natural science which would recommend either belief or doubt as a path to discovery. Some discoveries are prompted by the conviction that something is fundamentally lacking in the existing framework of science, others by the opposite feeling that there is far more implied in it than has yet been realized.

Manifestations of strain produced in other ways are, ordinarily, no less equivocal. When Rumford demonstrated the continuous production of heat in cannon-boring operations, we feel that the caloric theory was not merely strained but shattered. However, its adherents merely introduced a plausible readjustment of their premises when we say they should have capitulated. In an exactly parallel circum- 
stance, given the complete unobservability of the stellar parallax that is the one "certain" consequence of the moving earth he had postulated, Copernicus and his followers simply added to his theory the drastic assumption that the stars are so immensely distant that their parallactic displacement is immeasurably small. And we say this was indeed the assumption that Copernicus (and everyone else) should have made.

Is the situation clearer when strain develops from the discovery of entirely new kinds of data? The X-rays "accidentally" discovered by Roentgen seemed for a time to strain classical electromagnetic theory, but ultimately found snug accommodation within the theory. The photoelectric effect accidentally or incidentally discovered by Hertz (in the celebrated experiment by which he seemed to put that same theory beyond all possibility of doubt) we see in retrospect to have been a far more ominous anomaly. Yet at the time nobody saw it on the horizon as that portentous cloud no larger than a man's fist.

Even strain that develops as or from apparent antinomies within the structure of the theory may for long go unrecognized as such. The luminiferous ether, it transpires, must be more tenuous than the most rarefied gas, yet also more rigid and elastic than the strongest steel. Today we feel that 19th-century physicists erred in not recognizing this anomalous combination of properties as a symptom of strain. We, on the other hand, regard as only a passing difficulty the various infinities that crop up so awkwardly in quantum field theory. Will history approve our judgment?

Where there are but a few anomalies to be pronounced as such, or dissipated with supplementary assumptions, nobody may detect the presence of strain. A few sensitive souls may react to any greater accumulation of anomalies, if they are recognized as such. That remains ever a large if: even in our own day few indeed felt anything fundamentally amiss when a dense overpopulation of transuranic elements had to be hypothesized to account for the results of neutron-bombardment of uranium. Yet always by some the sense of strain is felt sooner or later, and sooner rather than later when discrepancies are striking and/or numerous. At that moment occurs the decisive transition Bartlett sketches with an apt example.

Some infectious diseases are bacteria borne; more are bacteria borne; very likely all are bacteria borne. But no; here is one, the mosaic dis- 
ease of the tobacco plant, which is not so. This is awkward, but so long as there is only one disease, and so long as there is, in a broad sense, only one method of study, nothing much is going to be done about it. As soon as other cases are found, and as soon as other methods find them, active work starts up again, and the great chances are that it will have new directions which may set the main stream of research for quite a long time.

Here is initiated a new endeavor, with its own characteristic orientations and potentialities. Here is born a new theory-probably rather crude and narrow, but promising-to enter upon the cycle I have sketched. Sometimes, as in the case of the virus theory, the new theory will merely supplement the old. But when instead it bids fair to supplant the old, the crisis of the old order is at hand.

The death struggle. The challenge of a new theory at once invests with new significance anomalies perhaps long disguised, or dismissed as unimportant. Precisely those anomalies, having in most cases supplied the primary stimulus to creation of the new theory, are likely to be highlighted at the center of its focal region. For the older theory to leave them still unexplained becomes then an overt mark of its insufficiency. Yet such explanation will ordinarily require one or more supplementary ad hoc postulates. The need for these will, if the new theory proves viable, be ever increased. That theory will lead us to a multitude of new phenomena. These remain still to be discovered precisely because the older theory has never given any hint of their possibility, and these are consequently just the discoveries likely to tax most cruelly that theory's correlative capacity. Now we see wide before us the way in which a heuristically powerful theory will, in quite short order, completely reverse a balance of correlative efficiency and explanatory appeal initially unfavorable to it.

Every time Mendeleev left a space in his periodic table he had to postulate an undiscovered element. With the subsequent discovery of those elements the ad hoc postulates are completely eliminated. Such new discoveries, the correction of apparently contradictory data (e.g., Priestley's "stubborn facts"), and the kind of conceptual refinements earlier noted will, all together, tend substantially to reduce the body of postulates required to constitute the new theory. Beyond such reductions in the denominator, the correlative index of that theory will be still further increased by complementary changes in the numerator. To the extent that some of the original exclusions 
are lifted by conceptual refinements, additions will be made to a numerator which will in any case be still more spectacularly enlarged by new discoveries. For example, at the outset only a very few known relations lent themselves to interpretation by Torricelli's new theory, which however led to the discovery of a great many new relations in barometry and elsewhere. These discoveries fit smoothly into the theory that predicted them. On the other hand, they can be accommodated in the theory of horror vacui only with the aid of supplementary postulates which decrease the correlative index of that theory. Thus, given the heuristic power of Torricelli's theory, it is almost automatically assured an ultimately superior correlative index.

Displays of heuristic power also affect explanatory appeal. When Newton postulates a universal gravitational attraction he asks us to accept a purely hypothetical force. Guided by Newton's theory, Cavendish at last detects the action of that force in the laboratory. What Newton proposes is then no longer strictly hypothetical, but only an immense generalization of what we can observe. In somewhat the same way, Copernicus' "absurd" postulate of a moving earth provokes multiple unsuccessful searches for stellar parallax. But these do lead to the detection of stellar aberration, which is at last some reasonably clear indication that the earth does have an annual motion. Such gains in the explanatory appeal of the heuristically powerful theory are complemented by losses in the appeal of its competitor. Copernicus' theory leads men to expect the phase cycle of Venus. That cycle discovered, the Ptolemaic system can account for it only with the aid of supplementary assumptions of no great plausibility.

Given the above processes, we may not be long in recognizing some difference between the number and character of the supplementary postulates required to support a theory in its decrepitude and those that for a time sustain a theory in its earliest youth. Thus we distinguish different kinds of weakness in the infant who pushes a chair in front of him as he learns to walk and in the ancient who drags himself around on crutches. Overloaded with supplementary assumptions, the older theory becomes functionally inadequate. That is, it becomes difficult to handle, its adherents begin to use it in different ways, and communication between them may begin to fail. Worst of all, any lingering remnants of heuristic power are impaired. The theory grows so complex that unequivocal predictions are not 
readily drawn from it. An accidental discovery may lead Cavendish to determine the composition of water, which he is able to construe in phlogistic terms. But Cavendish is totally unable to draw from the phlogiston theory any clues to further knowledge, while when Lavoisier hears of Cavendish's work he at once goes on to experiments producing new and exciting data.

The ultimate fate of the old theory, ever more heavily overburdened, is no longer open to doubt. Hear Nietzsche:

An old Chinese sage once said he had heard that when mighty empires were doomed they began to have numberless laws.

The death throes may now be abruptly terminated. Given grounds for dissatisfaction with the older theory no longer capable of discharging its functions, given also a promising alternative to it, the two essential preconditions for a crucial experiment now exist. Any who refuse to accept the verdict of that test will be progressively wiped out by the mechanism of natural selection described earlier. The older theory dies, the older opinion is falsified, a scientific revolution has taken place. Wrong, wrong, wrong! The old theory may live still (though on a different plane), it is not then falsified but only subordinated, and what has occurred in science is an intrinsically evolutionary development.

\section{Scientific Revolutions?}

In all revolutions we find some elements of continuity (e.g., the major social reorganization produced by a French or Russian revolution leaves the family grouping essentially intact). In the archetype of all evolutions we find an irreducible element of discontinuity (in organic evolution each mutation is an abrupt discrete step). We may nonetheless usefully distinguish revolutions, in which the greater number of the elements of paramount concern change sharply, from: evolutions, in which continuity seems the dominant quality of the focal elements. Considering the elements essentially characteristic of cosmology, we earlier concluded that there are cosmologic revolutions. Considering now the elements essentially characteristic of science, I propose to show that evolutionary continuity is so overwhelmingly dominant that the term "scientific revolution" is distinctly misleading. There are no scientific revolutions. Consider as 
examples the earliest and the two most recent of the scientific developments commonly so denominated.

Speaking as a cosmologist I cannot fail to recognize a Copernican Revolution: an older world view is completely shattered. But where is the revolution in science? Astronomers are newly reminded not to accept uncritically all that seems obvious to common sense. But for some 2000 years already astronomers had been busy seeking immutable regularity in celestial motions quite "obviously" irregular. Astronomers are newly counseled also to deploy fully the powers of mathematics, but that counsel had been audible, long before Eudoxus, even to the Babylonians. And, as for centuries before, so too after the Copernican "Revolution" astronomers continue to observe "objects" in the sky while continuing also their endeavor to fit "orbits" to those observations. They now essay some new kinds of orbits, with a new reference center; they look for some new things, and make somewhat novel interpretations of what they see. But in the practice of astronomy, what is the change aptly qualified as "revolutionary"?

An almost uninterrupted series of conspicuous successes had led the typical physicist of the late 19th century to regard classical physics as a completely secure foundation for the ultimate cosmology. The initial impact of Einstein's special relativity theory then seemed devastating. But, when the smoke cleared, Planck at least could detect no revolution in science:

The theory of relativity ... has proved to be the completion and culmination of the structure of classical physics.

Such "completion" seems peculiarly ill-suited to description as a revolution. Did Planck perhaps suffer from astigmatism? With a philosophic outlook very nearly the polar antithesis of Planck's, Bohr still saw this development in much the same way:

... the theory of relativity approaches, in a particularly high degree, the classical ideal of unity and causality in the description of nature.

\section{Einstein himself writes:}

With respect to the theory of relativity it is not at all a question of a revolutionary act, but of a natural development of a line which can be pursued [backward] through centuries. 
Long after microcosmic determinism was relinquished in scientific practice (e.g., in statistical mechanics), it was, we saw earlier, still maintained as a cosmologic absolute in principle. Quantum mechanics' denial in principle of such determinism does then produce upheaval in the sphere of cosmology. And in the sphere of science? Acquiring some powerful new tools of thought, we recognize that some of our older tools are not so omnipotent as we thought. Certain hopes are dashed, other new hopes are created. Scientific effort acquires a strong new impetus and a slightly different slant. Of the changes so made in the practice of physics, what is revolutionary? Weizsäcker declares:

It is the definitive difference of quantum mechanics from classical physics, that it cannot even enunciate its propositions without at the same time expressing the way in which they are known.

The establishment of this fact is just as natural with respect to the practice of classical physics, as it is revolutionary for the world-view not only of classical physics, but of most philosophical systems.

The growth of order. In nature, viable species produced by mutations may ultimately go on, through further abrupt mutations, to still other forms of life. One reason we term this saltatory mode of development an evolution is that we find long-term sequences of organisms which constitute steady progressions toward ever higher levels of organized complexity. Scientific history presents us with some strikingly analogous sequences of theories. We find in all recent cases that, however narrow in scope at its first appearance, a viable new theory ultimately stretches to accommodate at least as much as the theory it displaces, and generally a great deal more. In long-term perspective, then, we find a development of "higher" theories that, through constantly increasing correlative indices, contrive to hold firmly in order a body of scientific experience forever increasing in both size and complexity.

This progress is a fact, but how is it possible? Weizsäcker points the way with this comment:

. . . everything which is true at all in the physics of Aristotle, is contained in classical [Newtonian] physics, even if it is in part differently expressed; and we should expect the corresponding situation for the relation of our physics to every future one. 
When an older theory is found insufficient, practically all its holding of colligative relations will survive intact, rewoven in the pattern of the new theory. When the older theory is a comparatively primitive one, we readily understand how the few relations it accommodates might be rewoven in short order. But when we come to deal with the displacement of major theories, accommodating myriad relations, the reweaving of all the separate relations in a drastically different pattern appears no light undertaking. Indeed, were the relations wholly separated, and the pattern wholly different, this might well prove an almost impossible undertaking.

From the fact that the reweaving of even major theoretic patterns is completed reasonably promptly, we infer the presence of some further element of continuity in the sequence of scientific theories. To be sure, sometimes the older theory is not so much destroyed as -rather in the manner of the duck by Peter's wolf-engulfed entire by the new. Thus classical electrodynamics was esteemed beautiful at least in part because of the extraordinarily high value of its correlative index. In quantum mechanics we acquire a system of still higher correlative index which, Dirac suggests, incorporates the classical system virtually en bloc.

This is brought about by the fact that the changes made in the classical theory are very few in number, although they are of a fundamental nature and involve the introduction of entirely new concepts, and are such that practically all the features of the classical theory to which it owes its attractiveness can be taken over unchanged into the new theory.

The correspondence principle. Perhaps the most striking element of continuity in organic evolution is the survival in newer species of major parts of the structural organization found in the old. In the succession of recent scientific theories we find a similar profoundly evolutionary development. Sambursky emphasizes the generality of the relation of correspondence he considers to obtain between relativistic and classical mechanics:

In spite of the theoretical and philosophical difference between classical mechanics and the new [relativistic] theory, and in spite of the formal difference in their mathematical method, the former is still included in the latter as a first approximation. History of science in the 
past three hundred years is characterized by a chronological and almost organic sequence in its development which was not found to anything like the same extent in Greek science. It is above all the history of physics from Galileo to our time that makes us realize that science advances toward reality so to say by concentric approximations-each theory contains its forerunner as a "special case."

How can such correspondence of new and old possibly be attained when the new theory involves fundamentally different primitive concepts? Sometimes very simply indeed. Setting out from the premises of the new theory we may find that, making certain assumptions and/or approximations, we obtain certain derivative propositions identifiable with the premises of the older theory. In physical circumstances that invalidate the assumptions and approximations, the old theory must fail, and is superseded by the new. But, when the physical circumstances are compatible with those assumptions and approximations, the entire pattern of the older theory reappears almost intact, as the limiting form of the new. And that correspondence offers something Einstein and Bohr were perhaps the first fully to grasp: a potent new heuristic principle. Hutten comments:

It is the correspondence principle that shows us how to construct a better and more comprehensive theory, on the basis of a simpler and narrow theory, or of a model. The principle formulates the condition which the new theory must satisfy: there must be an asymptotic agreement between the main formulae of the old and of the new theory.

In constructing his first theory of the hydrogen atom, Bohr established the proper mode of quantization by imposing the condition that a relation of classical electrodynamics must appear as the limiting case of his own formula. The "scientific revolution" of quantum theory was thus in part achieved by accepting as a principle the evolutionary continuity of scientific theories!

Perhaps one may regard correspondence as a psychological phenomenon. James remarks that "new truth"

. . marries old opinion to new fact so as ever to show a minimum of jolt, a maximum of continuity. We hold a theory true just in proportion to its success in solving this "problem of maxima and minima."

But this continuity has also another root, in a logical requirement. In the foregoing chapter we observed that the latest member of a 
hierarchic series of more and more abstract theories acquires its physical relevance only as a member of an unbroken series. The demand for correspondence safeguards the integrity of that series. And the chronological succession of "revolutionary" advances in scientific theory is, thereby, fashioned into the risers of the perfectly continuous logical stairway by which alone we can descend from our "highest" theory to the ground level of experience.

However long we may choose to discourse on the intangible entities of quantum mechanics, a science of quantum mechanics must ultimately be brought to bear in the mode Oppenheimer describes.

The measurements that we have talked about in such highly abstract form do in fact come down in the end to looking at the position of a pointer, or the reading of time on a watch, or measuring out where on a photographic plate or a phosphorescent screen a flash of light or a patch of darkness occurs. They all rest on reducing the experience with atomic systems to experiment and observation made manifest, unambiguous, and objective in the behavior of large objects, where the precautions and incertitudes of the atomic domain no longer directly apply.

To these large objects we of course apply the concepts of classical physics and, as Heisenberg maintains, this application is forced on us.

The use of these concepts, including space, time and causality, is in fact the condition for observing atomic events and is, in this sense of the word, "a priori." What Kant had not foreseen was that these $a$ priori concepts can be the conditions for science and at the same time can have only a limited range of applicability. When we make an experiment we have to assume a causal chain of events that leads from the atomic event through the apparatus finally to the eye of the observer; if this causal chain was not assumed, nothing could be known about the atomic event.

There is an important point here. The older physics is not simply contained inside the new; the older physics continues, with undiminished competence, to function independently beside the new.

\section{NOT FALSIFICATION BUT SUBORDINATION}

When a new form of life appears in nature, it need not wholly displace the parental form(s). The emergence of amphibians substan- 
tially changes the balance of species in coastal waters, and leads to a wholly new colonization of the land. But the shark-a very ancient form of life-still continues his dominance of the oceanic deeps. A major new scientific theory, with a much enlarged scope of correlation, is well adapted to function throughout a vastly enlarged domain of experience. We may be shocked to discover that the older theory, to whose authority we formerly recognized no limits, is in certain regions completely insufficient. Within its wonted domain, however, it may conserve all its old effectiveness. Far from destroying classical physics, says Weizsäcker,

The thesis of quantum mechanics is, rather, the persistence of classical laws; it asserts: when any classically defined quantity is known through measurement, then all the consequences which can be drawn from this knowledge according to classical physics, are exactly correct.

In such cases the older theory is not supplanted, but only subordinated! Thus the overweening pretensions of a feudal lord are checked by a strong sovereign newly risen. Having made his submission, the feudal lord continues in full enjoyment of dominion over his traditional estate. Heisenberg remarks the parallel case in science:

It is not the validity but only the applicability of the classical laws which is restricted by modern physics.

Subordination rather than falsification may perhaps obtain even when there is no correspondence of new and old in the sense that links modern and classical physics. Given the everyday experience in the midst of which we are brought up, an older theory will often seem a "more natural" way of handling the phenomena it was originally designed to construe-which is no doubt one reason it is the older theory. Sometimes it will also offer a genuinely simpler way of dealing with those phenomena, and so quite understandably continue in use long after its subordination. Conceptual tools will then survive just as empirical tools survive: though today we measure "distance" by a multitude of methods unknown to Archimedes, the measuring stick remains useful to us as it was to him.

I am very far from arguing that all older scientific theories survive in one form or another: the eclipse of the phlogiston theory, for example, seems total. On the other hand, I have some suspicion that, 
when computing the fuel costs for a projected motor tour, one proceeds by way of the Aristotelian view that continued motion demands a mover; and not at all by beginning with the Newtonian concept of inertia, and then proceeding to make corrections for all items of frictional resistance. Even more to the point, observe the notable survival of the geocentric system "falsified" in the earliest of "scientific revolutions." The manifold relations originally conceived and expressed in geocentric terms are handled with matchless felicity in that frame of reference. And so to this very day the navigators and surveyors, who are most concerned with just those relations, continue to use the geocentric system. If, as I suppose, the geocentric system is not meaningfully a limiting case of the heliocentric system, then the correspondence principle does not apply. Nonetheless, the older theory remains active in the presence of the "new" theory by which it has been subordinated. Nobody "believes" the geocentric system in any cosmologic sense, nor does anybody so "believe" the caloric theory. Yet do we not still use the latter much as we do the former? Though we never mention a caloric fluid, in calorimetric(!) work do we really conceive ourselves as measuring motion or a substantive heat? A "heat pump" doesn't supply motion: a pump pumps something, if not heat then energy. What we conceive to fow or pass through a thermally permeable membrane is surely not motion but heat. Offering a felicitously "natural" conception of the simple thermal data it was designed to handle, the caloric theory remains still useful and in covert use. It has not been falsified, but only subordinated, by the far more widely applicable kinetic theory, of whicti it is not meaningfully a limiting case.

However it may be in these older instances, the phenomenon of incomplete displacement is perhaps the most conspicuous feature of the more recent "revolutions" to which the correspondence principle does apply. The advent of relativistic mechanics leaves classical mechanics in complete command of the classical domain. For this is, of course, just the domain of all those limiting cases that classical mechanics-as the limiting form of relativistic mechanics-handles very nearly to perfection. Thus, for example, the accuracy of purely classical celestial dynamics is perhaps best indicated by the magnitude of its one "serious" anomaly: the predicted rate of precession of the perihelion of Mercury fails by a margin that would represent an accumulated error of one degree of arc in 8700 years. Obviously, we 
do not even think of trying to derive the same results by the formidablv complex techniques of general relativity.

What is the status of a theory thus subordinated but not slain? A respected physicist who is also a philosopher of science writes: "Newton's theory became false when Einstein's was accepted." What has become "false" we would think must be discarded. Yet every day and everywhere scientists continue to use Newtonian physics, and find in it a tool of immense heuristic power. But when we speak of truth or falsity clearly we speak not of science but of cosmology; and it is solely as an element of cosmology that "Newton's theory became false. ..." In its own familiar realm, classical mechanics remains completely valid as a scientific theory. Einstein himself saw this very clearly when, with reference to his own work, he wrote:

No one must think that Newton's great creation can be overthrown in any real sense by this or any other theory.

The "problem" of dual description. Eddington asks us whether the hard, smooth-surfaced table perceived by the man of common sense is not "really" a thin haze of electrons and nucleons. We suppose there can be but one correct description of the "real object": yet here we are presented with two apparently wholly irreconcilable descriptions, each of which makes a very strong claim to consideration. So perplexed, we may begin to wonder whether both descriptions are in some way illusory, whether any description can be aught but illusory, and perhaps even whether there is any "real object" to be described. These are surely absurd lengths to go in the face of a pseudoproblem which arises simply from the co-existence of various more or less subordinate theories.

The situation is much clarified by the highly suggestive analogue proposed by Toulmin. Consider, he says, the alternate representations of the same region offered by two different maps: a road map and a physical (i.e., topographic) map.

. . . many things can be mapped on the physical map which there is no way of putting into the road map: this is a consequence of the ways in which the two maps are produced, and of the comparative poverty of the system of signs used on the road map. On the other hand, given the physical map, one could produce a satisfactory road map: all that appears on the road map has its counterpart on the more elaborate map, even though in a different form. But this does not 
mean that the road map is not, of its kind, an unexceptionable map of the region. Providing that it is not thought of as having irrelevant pretensions, there is nothing wrong with it: indeed, for some applications one will be able to discover the things one wants to know, e.g., distances by car, more easily from the road map than from the physical one.

The topographic map is useful in a wider range of applications; and the road map is derivable from it, as it is not from the road map. The road map is thus subordinate to the topographic map. But no greater degree of illusoriness then attaches to the road map, which effectively discharges precisely those functions it was designed for.

Let me suggest a different analogue. Flying at a considerable altitude, I observe some "objects" in a field below. Guided by earlier experiences, my companion the truck-driver declares that the objects are rectangular blocks, and my companion the farmer asserts that they are aggregates of strands of hay. Loading his truck, the truckdriver does indeed treat such objects as rectangular blocks; feeding his cattle, the farmer treats them as hay. Perhaps the concept of rectangular block is subordinate to the concept of hay: knowing something of the properties of hay, I can infer the possibility of forming and handling it in bales; knowing only the presence of rectangular blocks, I cannot infer that I am in the presence of cattle feed. But it does not then follow that there is anything deceptive about the concept of rectangular blocks.

I find only one non-fundamental distraction that makes the situation of Eddington's tables apparently different from the proposed analogues. The analogues suggest paired views of which one is directly subordinate to the other. Eddington's tables are the two extremes of a whole series of theoretic views and, as Conant observes, the omission of all reference to the intermediate members is what makes the extremes seem so irreconcilably far apart. Given the view of electrons and nucleons, I cannot pass at once to the everyday table; but I can understand how, in certain contexts, atoms of various kinds may be formed. Given such atoms, I can similarly understand the possibility of forming molecules of cellulose, lignin, etc. And so on from molecules to fibrils to fibers to wood to, at the very last, the familiar table of everyday experience.

All these views are, directly or indirectly, subordinate to the view of electrons and nucleons-which, in principle, encompasses all the 
ways we have found to conceive and handle tables. But we are under no obligation whatever to choose among these views. Each and every one is justified if it serves the purpose(s) for which it was shaped. We prize the "highest" (quantum-mechanical) member of the theoretical hierarchy for its immense scope of correlation, though it is in fact a rather notably unuseful way of viewing the table (save, perhaps, were one concerned to use that as an impromptu radiation shield). The "lowest" (common-sense) member of the theoretical hierarchy is unquestionably the most useful way of viewing the table as a table. Moreover, there is nothing at all illusory about this view. Indeed, Eddington's "higher" table simple cannot impugn the "lower" familiar view. For, as we saw earlier, the "higher" theory acquires physical meaning only by presuming the absolute validity of the "lower." And so, says Oppenheimer, we must recognize that-

. . . ever-increasing refinements and critical revisions in the way we talk about remote or small or inaccessible parts of the physical world have no direct relevance to the familiar physical world of common experience.

\section{EVOLUTION AND ERROR}

The emergence of higher forms of life in nature may be held to signify the insufficiency of older lower forms less competent to deal with some of the range of terrestrial habitats. But the higher forms develop only from or through the lower which, if they be "errors," are not for that the less essential to such development. The "higher" scientific theory-often less convenient in everyday use, but powerful enough to impose its rule throughout a drastically extended range of experience-develops similarly from and through older "lower" theories less generally sufficient, and "errors" at least in the degree they have been thought final.

Comte, Mach, and their doctrinal descendents have supposed that a science focused on the description, rather than on the explanation, of experience would be a science built lastingly-a body of positive knowledge enduring for the ages. But science seeks general knowledge of the world, and can therefore never hope to possess knowledge absolutely generally secure. To Comte's horror, Regnault finds inexactness even in Boyle's law-an apparently exemplary economic description of gaseous behavior, which makes no "vain pretense" to be anything more than that. If we thus encounter limits to the suffi- 
ciency of our laws, how much more likely are we to find limits to the sufficiency of our theories! That science might be spared the later discovery of error, Mach repudiated the atomic theory. But, as we saw in Chapter VII, identification of the "good" in science with the avoidance of error comes close to pure negativism. A "scientific revolution" is not the catastrophic revelation of corruption in the body scientific but, rather, the exuberant triumph of an undertaking that cannot approach relative truth otherwise than through relative error. Surely Popper is right in insisting that:

The old scientific ideal of epistēme- of absolutely certain, demonstrable knowledge-has proved to be an idol.

. . . Bold ideas, unjustified anticipations, and speculative thought, are our only means for interpreting nature: our only organon, our only instrument, for grasping her. And we must hazard them to win our prize. Those among us who are unwilling to expose their ideas to the hazard of refutation do not take part in the scientific game.

Of those so declining, a few become logicians and epistemologists apparently ill content with their own honorable traditional titles. Such a "philosopher of science" may, quite understandably, still cherish the logician's ideal of an analysis that is tidy, complete, and definitive. Thus Reichenbach considered it quite proper that:

The philosopher of science is not much interested in the thought processes which lead to scientific discoveries; he. looks for a logical analysis of the completed theory, . . . That is, he is not interested in the context of discovery, but in the context of justification.

In this chapter we have found heuristic power the focal criterion of judgment of a scientific theory. The theory's effectiveness as instrument of discovery is the supreme justification for its acceptance by scientists. For them the "context of justification" is included within and inseparable from the "context of discovery." If he accepts the Reichenbachian dichotomy, the "philosopher of science" no longer concerns himself with the science practiced by scientists. Sometimes of course he proudly declares as much-as in the following statement which, for me at least, has a ring ominously reminiscent of scholasticism, in the most pejorative sense of the term.

After all, this model [of scientific explanation] is not supposed to be a model of actual scientific practice; it is an ideal to which scientific practice is supposed to conform if it is to be satisfactory. Hence, if a 
difference is observed between the model and scientific practice, this is to be regarded as a criticism of scientific practice, and not of the model.

Cherishing the ideal of a tidy, complete, and definitive account of some organism, one might think to confine himself to a careful analysis of its skeletal remains-leaving out of all account the complex, confusing, and "untidy" activities of the living flesh. But we never delude ourselves that palæontology is a complete biology. That logical analysis of completed scientific theories offers a complete philosophy of science is a delusion no better founded. Indeed, if we confine ourselves to logical analysis of finished structures, our understanding must forever rest incomplete simply because we cannot then understand why those structures are what they are. Poincaré emphasizes that this consideration applies even to the purely formal discipline of mathematics-and he drives his point home with the metaphor of

. . . those delicate assemblages of silicious needles which form the skeleton of certain sponges. When the organic matter has disappeared, there remains only a frail and elegant lace-work. True, nothing is there except silica, but what is interesting is the form this silica has taken, and we could not understand it if we did not know the living sponge which has given it precisely this form.

The logician's dream of a definitive philosophy of science is nothing but a dream. Through the mesh of epistemologic analyses, conducted exclusively in the hypothetically distinct "context of justification," slips all that makes science what it is: a unique enterprise best characterized by that extraordinary dynamism of which I have sought to render just account. 


\section{CHAPTER $\mathrm{X}$}

\section{Organized Science}

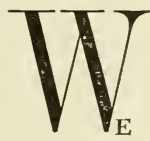

devices of organized science: the scholarly periodical, the international conclave, and so forth. We can see also that these are devices expressly designed to further two specific functions-communication and education-which absorb much of the attention and most of the funds of the professional organizations of scientists.

Through education the scientist learns the achievement of his predecessors, which is the point of departure for his own endeavor. He learns, too, the esoteric terminology he will use in exchanging with his contemporaries communications of scientific intelligence that are both comprehensive and comparatively unequivocal. These can be comprehensive because of the immense condensation of what needs to be communicated. Thus, for example, I need not report the actual heights in my manometer, nor the circumstances of ambient temperature, barometric pressure, and geographic location in which those heights were measured. Instead I report only the pressures derived from all these data, and my report is essentially unequivocal because today scientists do thoroughly understand what is meant by "pressure." The older scientific literature is impaired by a great variety of maddening obscurities and ambiguities. Today such uncertainties are suppressed by uniform adoption of nomenclature, units, conversion factors, and standards suggested by international commissions of scientists representing the learned societies directly concerned.

With the preparatory education of scientists the modern profes- 
sional societies concern themselves only rather secondarily-to the extent of awarding fellowships, appointing committees on accreditation, curriculum reform, and so on. Very much as was the Royal Society, the earliest of all scientific organizations to survive to the present day, the modern professional societies are primarily concerned with communication, and thus with the continuing mutual education of scientists. These societies arrange meetings, support journals and abstracting services, sponsor monograph series and, through such devices of communication, contrive to link scientists all over the world in an immense corporate endeavor. But these devices of the professional societies, though essential, no more suffice to make organized science than the devices of company and regimental commands suffice to make of an anarchic rabble an army. We conceive an élan or esprit that makes the army, and the soul of organized science inheres in a cultural tradition that inspires and directs all members of the community Nagel describes as follows:

Viewed in broad perspective, science is an enterprise carried on by a self-governing community of inquirers who conduct themselves in accordance with an unwritten but binding code. Each member of this republic has the right and the obligation to make the most of his capacities for original and inventive research, to make full use of his powers of imagination and insight, to be independent in his analyses and assessments, and to dissent from the views of others if in his judgment the evidence requires him to do so. In return for this, on the other hand, he must submit his own investigations to examination by his scientific peers, and he must be prepared to defend his claims by reasoned argument against all competent critics, even if he should believe himself their superior in knowledge and insight.

\section{The Invisible College}

The Royal Society of London was formed by a group of "virtuosi" who shared an interest in science and a strong conviction that scientific knowledge can best be advanced by experimentation. Chartered in 1662, the Society survived, I think, because of that happy conception of scientific organization reflected in the name by which some of its earliest members referred to their then-informal association: the Invisible College. The essence of a college is not its physical plant, trustees, administration, departmental organization, nor even 
its student body. The unique cachet of the institution derives from the community of scholars that graces it. The Invisible College of science is the self-constituted association of scholars who-banded together in mutual regard for stimulation and assistance-seek the advancement of a communal ideal that, says Nagel, enjoins rejection of every system of dogma while at the same time demanding faithful adherence to the socially cultivated standards of sound workmanship.

Observe that rejection of all commitment to dogma entails the rejection in science of all human authority, always exercised according to the dogmatic preconceptions of those who wield it. The motto adopted by the Royal Society, Nullius in verba, expresses the empirical orientation of its members; but expresses, too, their rejection of any authority beyond that of nature, expressed in the results of experiments. To be sure, the Society had its officers, but (as in the modern professional society) these exercised only relatively small powers delegated to them. Quite characteristically, this is a system of atomic sovereignty.

Polanyi contrasts systems of general authority, which leave large scope to individual initiative and judgment, with systems of special authority like that of the Roman Church. The systems of general authority acknowledge traditions held excellent but still further perfectable. The systems of special authority are designed to preserve from all corruption traditions held already to have achieved their ultimate perfection. The Invisible College's is a system of general authority, and its tradition is carried on by a process that Polanyi well calls "creative renewal."

. . . a traditional process of creative thought cannot be carried on without wholly new additions being made to existing tradition at every stage of transmission. . . .

. . . there are always borderline cases requiring a measure of discretion, and even in routine cases there will often be an element of finer discrimination involved where a personal judgment is indispensable. The major principles of science, law, religion, etc., are continuously remoulded by decisions made in borderline cases and by the touch of personal judgment entering into almost every decision. And apart from this silent revolution steadily remoulding our heritage, there are the massive innovations introduced by the great pioneers. Yet each of these actions forms an essential part of the process of carrying on a tradition. 
Conjoined within the individual is both high respect for traditional belief and a strong sense of his freedom to challenge and amend it. In him there is a continuous dialogue of inspiration with conscience. The possibility of progress develops as the voice of inspiration can be heard. The stability of the tradition is assured because the voice of conscience is never stilled.

The structure of the Invisible College mirrors the typically loose noncompulsive organization of a Utopian socialist community. Or thus, at least, most scientists like to think of their association. Scientific organization as it actually exists is, to a remarkable degree, a faithful realization of the scientists' ideal of organization. However, as we proceed now with a sketch of this ideal, we shall find that-in order to render just account of the actual-we must from time to time interject certain qualifications of the ideal.

\section{THE COMMUNAL ADMINISTRATION, CONSENSUS, CULTURE}

In the Invisible College one finds no "core" of organization and authority. The affairs of science are administered by a great multitude of committees. The vast majority of these are completely informal, and have only a transient existence. Few if any of these committees function as ultimate governing boards. They serve rather as agencies for broad canvassing and systematic codification of expert opinion. In this way, Polanyi notes, an important concentration of authority will develop even in a culture that rejects all centralization of authority.

Authority is not equally distributed among scientists. There is a hierarchy of influence; but exceptional authority is attached not so much to offices as to persons. A scientist is granted exceptional influence by the fact that his opinion is valued and asked for.

Consider as an example the operation of the learned journal of some professional society. Control is vested in a publications committee and, in most cases, is actually exercised by a single editor selected by, and answerable to, the committee. But, in running his journal, the editor will call into consultation a large body of reviewers, or "referees." Each manuscript submitted for publication will be checked by two or more investigators known, through their publications, for their interest and competence in the field concerned. 
The referees will submit to the editor reports embodying specific criticisms and a general recommendation to publish or reject the manuscript. Most often the editor then returns to the author his manuscript, the referees' comments (ordinarily unsigned), and any comments the editor may himself wish to add. The author is expected either to accept and act upon the criticisms or else to show how, in his opinion, they are unjustified. The editor will then himself review the situation. In difficult cases he may call for reports from additional referees (including, perhaps, some suggested by the author), or for further changes or justifications to be made by the author. When at last reached, the final decision is then less a matter of individual fiat than the consensus of an expert group.

This is the pattern of scientific administration generally. The award of a doctoral degree will ordinarily depend on the judgment of an examining committee drawn from the local academic community, but consultation with an outside authority is not uncommon. Appointments to important posts are made similarly, on the recommendations of local committees-which will, however, base their decisions on nominations and testimonials solicited from a much wider group of those competent to appraise the candidates and their works. Committees concerned with award of prizes, fellowships, research grants, and so forth function in much the same way. In no one case is there a general consultation of the entire electorate: the "voters" are always, for the purpose in hand, a relatively small elite group of the specially qualified. But the sum of all administrative judgments is, effectively, constituted from some opinions of practically everybody competent to judge anything-and, as Polanyi notes, these judgments generally command remarkably wide approval.

Two scientists acting unknown to each other as referees for the publication of one paper usually agree about its approximate value. Two referees reporting independently on an application for a higher degree rarely diverge greatly. Hundreds of published scientific papers pass review of thousands of scientific readers before any of them finds reason to protest against the insufficient standard of a paper.

This impressive feat of communal administration is of course founded on a communal consensus of belief no less impressive. Scientists take it quite for granted that they should agree. Polanyi properly stresses that: 
The consensus prevailing in modern science is certainly remarkable. Consider the fact that each scientist follows his own personal judgment for believing any particular claim of science and each is responsible for finding a problem and pursuing it in his own way; and that each again verifies and propounds his own results according to his personal judgment. Consider moreover that discovery is constantly at work, profoundly remoulding science in each generation. And yet in spite of such extreme individualism acting in so many widely disparate branches, and in spite of the general flux in which they are all involved, we see scientists continuing to agree on most points of science. Even though controversy never ceases among them, there is hardly a question on which they do not agree after a few years' discussion.

Often, of course, a dispute can be brought to focus and promptly resolved by some crucial test. But, as we saw in the preceding chapter, such a test can resolve one issue only because all the disputants already agree on everything else involved in making the test. This same factor remains active even when no crucial test can be devised. The area of disagreement is shrunken, ultimately wiped out, by a "surface tension" forcing closure within the vast area of agreement by which disagreement is encompassed. The successes of communal administration are thus only the flower of a communal consensus that, I suppose, must spring from the cultural root indicated by Snow when he writes of scientists as follows:

. . . the scientific culture really is a culture, not only in an intellectual but also in an anthropologic sense. . . . there are common attitudes, common standards and patterns of behavior, common approaches and assumptions. This goes surprisingly wide and deep. . . .

. . . In their working, and in much of their emotional life, their attitudes are closer to other scientists than to non-scientists who in religion or politics or class have the same labels as themselves.

... Without thinking about it, they respond alike. That is what a culture means.

\section{THE NORMATIVE FUNCTIONS}

In the Invisible College laws must seem out of place. But even in ordinary colleges the dean only rarely (and generally ill-advisedly) feels impelled to act as a police officer. The effective function of a faculty is ordinarily secured simply by the action on its members of 
normative forces no less powerful for all that they are unenshrined in a legal code. The normative forces are not laws but customs, the mores of the community. And for the scientist his peers function not as policemen, judge and jury, but rather as a family, tribe or fellowship that acknowledges certain canons of "good taste." These canons are not unduly restrictive, and the individual may be disposed to accept them simply as a matter of enlightened self-interest. Thus, though this acceptance demands something of him, he recognizes that general acceptance of these canons will yield him rather more in return.

Protection. What are some canons of taste? Dishonesty, plagiai ism, and gross irresponsibility are, of course, in the worst of taste. Such trespasses are comparatively rare-because punishable by ostracism from the community in which most scientists value their membership. Since science can flourish only in an atmosphere of mutual confidence, polemic arguments impugning the intelligence or integrity of one's opponents are in bad taste. Mere absence of desire to reach agreement-evidenced in stubborn misunderstanding or misrepresentation of an opponent's position, or one's own-is in very bad taste, because it threatens the precious possibility of a communal consensus. Polanyi correctly notes that the tone of scientific debate, if not always one of sweet reasonableness, is generally held to a fairly high level by the discrimination of the audience addressed.

Controversies between leaders of thought are usually conducted in order to canvass supporters rather than to convert each other. Fairness and tolerance can hardly be maintained in a public contest unless its audience appreciates candor and moderation and can resist false oratory. A judicious public with a quick ear for insincerity of argument is therefore an essential partner in the practice of free controversy. It will insist upon being presented with moderate claims admitting frankly their element of personal conviction. It will demand this both in order to defend the balance of its own mind and as a token of clear and conscientious thinking on the part of those canvassing its support.

Today, for example, it is extraordinarily bad taste-because a threat to the integrity of the community-to appeal to extra-scientific considerations (e.g., religious or economic dogmas) on which all scientists cannot agree. Such arguments were apparently countenanced in scientific circles as little as a century ago, at the time of Darwin. But 
mores change with social evolution, of course, and today these arguments are held in bad taste-and in the very worst of taste when they are carried outside the community of science.

Accepting these restraints on his freedom, the scientist secures to himself the protection given by the community of others who accept the same canons of good taste. Thus, for example, he can rely on finding supporters for a justifiable claim to priority of discovery that is threatened by apparently unscrupulous competitors. Remarking that such support is often characterized in equal measure by altruism and passion, Merton comments that:

... the expression of disinterested moral indignation is a signpost announcing the violation of a social norm.

. . . scientific knowledge is not the richer or the poorer for having credit given where credit is due; it is the social institution of science and individual men of science that would suffer from repeated failures to allocate credit justly.

To provide for due recognition of individual achievement, the institution of science provides measures that run the entire gamut from the (infrequent) headlined priority dispute to the (commonplace) insistence of editors and referees that the relevant antecedent achievement of another be recognized in a footnote to a manuscript submitted for publication. Institutional protection also offers the individual assurance that he can argue for his scientific views without making himself a target for polemic abuse. Nor need he fear that his opponents in scientific controversy will seek to turn against him the coercive pressure of an outside authority (as Lysenko turned against Vavilov the whole political apparatus of his country). And valuing this protection afforded by the ivory tower, the scientist is, with his fellows, committed to defense of that bulwark.

A member of a college faculty who appeals the verdict of his colleagues to the institution's board of trustees shows something worse than just "bad taste." To his colleagues (perhaps even to himself?) he seems a traitor: he appeals for outside aid against the community of which he has been a member. By his action all are threatened: once the board of trustees is drawn in, where will they cease to meddle in matters in which they have no competence whatever? A scientist who appeals beyond the Invisible College to the general body of society, which is board of trustees to science, "loses face" and 
endangers all. For all will suffer from the irrationalism and the considerations of social, political, and economic expediency that become effective when even an "enlightened" modern society is drawn into scientific disputes. And so all are disturbed and rallied to vigorous action when, for example, political action seeks to overthrow the judgment and careers of scientists appraising the effectiveness of a battery additive.

Selection. The main defenders of scientific "good taste" are the editors and referees of scientific journals. It is their office to detect, and reject, incompetent reasoning and experiment; to discourage triviality, irrelevance, and arguments metaphysical and polemical; and to encourage, indeed demand, responsiveness to what seems legitimate criticism. Ultimately the author meets the demands made on him, persuades the editor of the justice with which he ignores some of them, or has his manuscript rejected. In the last event he may submit it to another journal controlled, he thinks, by less "captious" editors and referees. General opinion, however, may well hold that they are not simply less captious but less discriminating, and publication in a secondary journal is unlikely to command wide and immediate attention.

The author cannot ignore the suggestions of editors and referees. For him publication in a prestige journal is often a necessity. Particularly today, when the conduct of his research may demand huge sums of money, he is driven to seek, through worthy publications, the respect of peers any of whom may be called in as consultants on his applications for support. Quite beyond this, it is only the reputation established by published results that can secure for him a desirable appointment, the award of fellowships or prizes, remunerative consultancies, and so forth. Thus all the selective functions operative in the scientific community strongly sensitize the author to "public opinion," as expressed in the dicta of editors and referees. However, it is a mistake to suppose that, through such pressures, the author is forced into submission by external pressures alone.

As is clearest when we consider the behavior of men whose positions and reputations are already completely secure, authors generally want to conform with the cultural norm. After all, if one somehow contrives to pass off a shoddy piece of work, any transient advantage to him must inevitably terminate in the open rebuke, administered through others' publications, of experimental values 
and/or theoretical analyses that humiliatingly expose his incapacity. Moreover, though on occasion the author may feel himself constrained and even injured by the impositions of editors and referees, he will always agree that exacting standards of professional workmanship must somehow be maintained. As James observes:

Truth lives, in fact, for the most part on a credit system. . . . just as banknotes pass so long as nobody refuses them... . . You accept my verification of one thing, I yours of another. We trade on each other's truth [and truthfulness]. But beliefs verified concretely by somebody are the posts of the whole superstructure.

Thus, though the investigator may himself verify a few critical items he has some reason to doubt, to get ahead at all with his own work he must take on faith the soundness of the vast bulk of what is published by others in reputable journals. He cannot then wholly repudiate that selectivity of editors and referees on which he himself so heavily relies.

For the scientist's sensitivity to criticism we have now found two quite practical reasons. But the ultimate factor probably is, as Conant suggests, sociological.

The traditions he inherits, his instruments, the high degree of specialization, the crowd of witnesses that surrounds him, so to speak (if he publishes his results)-these all exert pressures that make impartiality in matters of his science almost automatic.

Never quite impartiality, for surely Trotter is correct in insisting that:

The dispassionate intellect, the open mind, the unprejudiced observer, exist in an exact sense only in a sort of intellectualist folklore; states even approaching them cannot be reached without a moral and emotional effort most of us cannot or will not make.

Nevertheless the very existence of the Invisible College must strongly promote this effort. Already educated to the impossible task of clearly distinguishing observation from inference, evidence from intuition, accomplishment from inspiration, the scientist feels goaded to make a case that will convince all doubters. In the conduct of his research he seeks to anticipate the criticisms of his peers by maintaining a high standard of workmanship. In shaping the presentation of his research, his consciousness of the discrimination of his peers not only makes him responsive to their criticisms but actively drives him, as Whe- 
well observed a century ago, to an effort of discrimination of which he might not otherwise be capable.

The authors and asserters of the new opinions, in order to make them defensible, have been compelled to make them consistent; in order to recommend them to others, they have been obliged to make them more entirely intelligible to themselves.

Appreciation. The artist may well ignore his critics: they do not paint. But the scientist's critics are also scientists, and he covets their good opinion. The public at large may be totally indifferent, scornful, or even hostile toward what most engages his interest and effort. However sympathetic, his family and nonprofessional friends are usually incapable of understanding, and so of truly appreciating, his achievements. Such appreciation he must and does seek from his professional colleagues. Perforce a snob, he is all the more so in that, even within the limited group of his fellows, he values most the esteem of those he himself most admires. And this is of prime importance: simple conformity and routine performance will not evoke the esteem of the most illustrious in the scientific community. In a statement highly characteristic in its use of the word "conquer," Pasteur asserts:

The recompense and the ambition of a scientist is to conquer the approbation of his peers and of the masters whom he venerates.

Equally characteristic, in its unaffectedness, is Darwin's statement to the same effect.

My love of natural science has been steady and ardent. This pure love has, however, been much aided by the ambition to be esteemed by my fellow naturalists.

We have then perhaps already identified that ambition of which Nicolle writes:

Without ambition and without vanity nobody would enter a profession so contrary to our natural appetites.

The scientist's vanity? Surely just that gratified in the making of an important original discovery.

Today the scientist's ambition and vanity may well be tempered by rather less elevated considerations. The supports and rewards for scientific work have ballooned in the last score of years. Thus the 
scientist's vanity may come to look beyond scientific success to the material rewards for such success. And his ambition may look beyond "the approbation of his peers" to the immense research grants their good opinion may win him. As the stakes constantly go up, the priority dispute is waged with intensified bitterness and unscrupulousness. As the pace of scientific research constantly quickens, the investigator strikes an increasingly precarious balance between the thoroughness required to avoid irresponsible work and the speed required to avoid work unpublishable because a competitor gets his results out first. It seems unlikely that the Invisible College can sustain these growing stresses without some ultimate change in its fundamental constitution. On the other hand, one does well to remember that, if today much intensified, these particular stresses are not at all a novelty in science.

Communication between the alchemists was poor because there was less desire to communicate than to boast of one's secrets. A residual secretiveness is still detectable at the beginning of modern science: the first "communication" of certain discoveries of Galileo, Huygens, and Hooke are by them given in the form of almost undecipherable anagrams. But long before the publication of scientific results could win any tangible reward, scientists had learned to want such publication-and of course science as a whole profits from that desire. Making an important scientific discovery, one enjoys something of the rapture of creation. Publishing that discovery, one makes claim on the appreciation of one's contemporaries and, even if they should prove entirely incompetent to understand and appreciate, a claim on the remembrance of generations of one's successors. Losing priority of publication, this wisp of immortality is lost, and the rapture is turned to bitterness by the realization that one's labor was wholly superfluous. And so of course long before first publication of results could win any tangible reward, scientists showed intense concern, and hot rivalry, for priority of discovery and publication. The "sacred fury" that powered the lifelong endeavor culminating in Kepler's discovery of his Third Law is today perhaps somewhat soiled, and certainly more discreetly veiled. But I believe that throughout the modern era something of that fury has united scientists in a common endeavor while also dividing some of them in the struggle to be such a chief priest of the cult of discovery as was Kepler himself. 
. . . I will indulge my sacred fury; I will taunt mankind with the frank confession that I have stolen the golden vases of the Egyptians, in order to build of them a tabernacle to my God, far indeed from the bounds of Egypt. If you forgive me, I shall rejoice; if you are angry, I shall bear it; the die is cast, the book is written, whether to be read now or by posterity I care not; it may wait a hundred years for its reader, if God himself has waited six thousand years for a man to contemplate his work.

\section{THE CO-ORDINATION OF EFFORT}

The indicated normative functions are all alike in one crucially important respect: they leave the individual free to choose his own line of research. The protective function is in part designed to guard just this freedom. The appreciative function merely encourages the individual to the best effort of which he is capable. The selective function has, to be sure, some effect prior to the initiation of research: the individual's position, facilities and funds perforce affect the work he can undertake. But, in science, position, funds, and facilities depend less on the individual's plans for future research than on his past accomplishments. Hence even here the normative action turns on what has been done, not on what is projected. The individual's freedom in the initiation of research may seem to negate all hope for a co-ordination of scientific effort. But, operating within the framework of the normative influences we have examined, this laissezfaire system seems in fact to produce a very well co-ordinated endeavor.

Conserve scientific effort by outlawing "irresponsible speculation"? But how, save through somebody's "irresponsibility," can everybody's "indubitability" be shown insufficient? The indifference, and even hostility, with which important results of speculative inquiry have been received, by the most illustrious scientists, leaves no ground for the illusion that, given authority, they would have permitted the initiation of any such inquiry. Judged by their issue, most numerological investigations are irresponsible and worthless. In retrospect we see extraordinary value in some such investigations, e.g., those of Kepler and Balmer. How to strike a nice balance between the certainty of loss and the possibility of gain? Modern science contrives to do so automatically-by the simple expedient of denying 
publication in professional journals (and thus all possibility of professional rewards) to speculations that fail to lead to some demonstrable success. The door is forever held open to anyone with confidence in his inspiration, and in his capacity to make it "pay off." The successes of a few such individuals will encourage other hardy souls to similar efforts; the dismal failures of most speculative inspirations will continue to discourage the vast majority. No bound is set to progress founded on speculation, yet the losses incurred through speculative extravagance are effectively minimized.

Conserve scientific effort by outlawing "wasteful duplication," particularly of experimental inquiries? But how else detect the spurious "established fact"? How many times was it observed (and how many times confidently asserted in textbooks) that the rare gases are totally inert-until, in 1962, xenon was found to form a variety of compounds? Most duplicated experiments would no doubt be waste effort, but there is little such waste. Few undertake such experiments simply because, if the same results are again obtained, the work is utterly unpublishable. Loss is thus limited, but the possibility of gain is preserved. One man may find what is to him alone a convincing reason to suppose that some earlier finding cannot be right, or that there was something more to be seen than was looked for. He is completely free to repeat the earlier work and, though of course he may still fail, he may also discover something not otherwise discoverable.

Maximize the rate of scientific progress by constraining scientific work to certain "promising lines"? But who could foresee the great promise of the rather routine confirmatory study that yielded Hahn and Strassman the result which opened the way to the discovery of nuclear fission? And, even given the individual's free choice of problems, is there not already a striking concentration of endeavor as delicately responsive to the potentialities for progress as it is completely spontaneous? From routine potboilers the scientist derives neither satisfaction nor reputation. In quest of both, he naturally endeavors to exploit what seem the most powerful new empirical devices and theoretical concepts. The gold-rush behavior noted in Chapter VI then automatically produces the desired concentration of activity. Yet while most scientists rush off to seek their fortunes in new lands, a percipient individual remains free to seek-and he sometimes strikes-oil in his own backyard. Change the metaphor: imagine human inquisitiveness pent up behind barriers to progress. Local 
penetration being made at some weak point, the discharge of a torrent of investigative energy tears wide the gap, and spreads out beyond it. Scientific judgment is expressed in the funneling of effort into the newly created gap. But the original penetration is often a discovery made by accident: the work of an apparently aimless wave or wavelet-one among many which earlier broke everywhere against a still unyielding barrier, and which still so break save at the breach.

The scientist's freedom in the initiation of research assures some matching of problems with interests, capacities, and enthusiasms. This is no trivial matter of the scientist's "happiness." Scientific work often demands long-suffering perseverance in the face of an almost endless series of disappointing checks. The chances of success are then much increased by the scientist's interest in, and commitment to, the problem he has himself chosen. Remarking how very deeply the individual may come to identify himself with his own hypothesis, Beveridge adds that:

Knowing this, the tactful director of research tries to lead the worker himself to suggest the line of work and then lets him feel the idea was his.

Under the laissez-faire system of the Invisible College this artifice is wholly unnecessary.

All arguments for a laissez-faire policy in science may seem invalidated by the many telling attacks made on laissez-faire economics. But the two contexts are utterly disparate in one crucial respect. Some forecast can be made of the course of economic development, but the course of scientific development may well encompass not only the unforeseen but even the previously unimaginable. We may, perfectly reasonably, deplore the tragic waste of men and resources in unproductive scientific work. But the few outstanding successes are product of the same process as the many undistinguished failures. We can no more legislate the one without the other, Holton implies, than we can decree the occurrence of progressive but no regressive mutations.

\section{The Super-Personal Intelligence}

One cannot but agree with Reichenbach that

The social character of scientific work is the source of its strength;

. . . the resultant of the contributions of the many intelligent indi- 
viduals is a sort of super-personal group intelligence, which is able to discover answers that a single individual could never find.

A uniform acknowledgment of one cultural tradition ensures the coherence of scientific endeavor, but its super-personal power derives from the diversity of individual capacities, skills, temperaments, and commitments. Kepler thought that:

The roads that lead men to knowledge are as wondrous as that knowledge itself.

Science achieves its maximum development only when there are as many different kinds of scientists as there are different kinds of roads to be explored.

Complementarity of capacities. Scientific organization, Darwin notes, alone makes possible the vital services rendered, by the host of the comparatively mediocre, to the few men of genius whose achievements give meaning to the work of all the rest. Newton said he saw further because he could stand on the shoulders of giantswhich they were, though beside him they seem to us pygmies. Just so, there is no Lavoisier without an antecedent Hales, no Einstein without an antecedent Mach. We write the history of science in terms of its great men, but lesser lights prepare the way for the great, whose work they also complete. Thus, to consolidate the deep but lightly held breakthrough initially made by the strategic insight of genius, we need multiple small enveloping operations carried through with the methodical skills of tacticians.

The sickly Pascal's insightful contribution to the establishment of Torricelli's aerostatic theory was the dispatch of a Perier, sound of wind and limb, to carry a barometer up the Puy de Dôme. That experiment completed, one might still wish to confirm Perier's results by carrying other barometers up other mountains. One might want also to venture up higher mountains, to see if the diminution in pressure continues-and perhaps up mountains closer to the equator or to the poles, to see if the effect varies with geographic position. Certainly one would want to improve the precision of the measurements, in order to investigate in detail the functional dependence of pressure on altitude. And so on. This work completes Torricelli's conceptual system, but the role of imagination ever contracts, and the character of those attracted to such work changes. Yet even the 
purely routine consolidation of one great success may accumulate the minor inconsistencies and critical commentaries that open the way to the next.

Complementarity of skills. Brahe, virtuoso observer, collects the data used by the virtuoso calculator, Kepler. The imaginative experiments of Faraday fire the theoretical imagination of Maxwell. Observe also the English school of empiricists thinking in terms of crude models (deriving perhaps from Bacon and represented by, say, Young), and the French school of theoretical formalists (deriving perhaps from Descartes and represented, say, by Fresnel): the weaknesses of each are cancelled by the strengths of the partner. The otherwise impossible may also be brought about by the joining of the skills of men working in different fields. With limited skill in chemistry, Fleming was unable to isolate penicillin or otherwise to exploit his discovery. That isolation and exploitation were the achievement of the quite heterogeneous skills pooled in the collaborative endeavor of a large group at Oxford.

Complementarity of temperaments. Only rarely do we find scientists who-like Galileo, Liebig, and Pasteur-are temperamentally equipped for success both as scholars and as controversialists. (How richly endowed was Leonardo and, in his loneliness, for how little did his work in science count!) But, through organized science, Dalton finds in Thomson a champion of the atomic theory; Darwin finds in Huxley a superb propagandist for the theory of evolution by natural selection. And the power that grows from the diversity of scientific temperament reaches far beyond this. Thus Berzelius, impatient to penetrate to the very heart of chemistry, correctly identifies its fundamental problem with characterization of the linkage that holds atoms in molecules. But in the second quarter of the 19th century this was a refractory problem with which Berzelius wrestled in vain. At that time chemical knowledge was most effectively advanced by men who, prepared blandly to ignore the ultimate problem, contentedly pursued with immense success non-ultimate inquiries into the number and arrangement of atoms in molecules.

Science has urgent need for both the kinds of seeing of which Koestler writes:

Science emerges in the shape of Janus, the double-faced god, guardian of doors and gates: the face in front alert and observant, while the other, dreamy and glassy-eyed, stares in the opposite direction. 
The critical scepticism of Mach and Poincaré thus prepares the way for an Einstein led on by an almost childlike vision of a harmonious universe. Relative to the alleged scientific method, Dingle remarks that:

. . . the method of arriving at the principle of least action was to prove that God works in the most economical way, and the method of finding a relation between the wave-lengths in the hydrogen spectrum, from which the whole of modem atomic theory has proceeded, was to hand the figures to a numerical mystic innocent of physical knowledge, ... .

The case of Maupertuis may perhaps be dismissed; he belonged to the youth of science. But Balmer's work was done in the latter part of the 19th century, when physicists were amply sophisticated-far too sophisticated-to think to look for what Balmer found: a relation that later became the focus of Bohr's first quantum theory of the atom.

Complementarity (and cancellation?) of commitments. Advancing toward truth held as a group consensus, science proceeds by rejecting error rendered easily recognizable as such by the diversity of commitments within the group. There is no paradox. Consider that the best grounds I can find for the rejection of error will lie in the antecedent labors of those men most firmly committed to the truth of what I would hold error. The fallibility of an opinion is made manifest, and corrigible, through the failure of those who will surely have left undone nothing that could possibly sustain the opinion. Men long dedicated themselves to the construction of perpetual motion machines of the first kind, and long failed. Given this failure of the most earnest efforts, we take as principle the conservation of energy, which is then not improperly described as a "principle of impotence."

Operating within the immense context of scientific agreement, the diversity of individual commitment powerfully promotes the attainment of impersonal knowledge useful to all regardless of their commitments. Our best assurance that we have made some approach to the hypothetical "naked fact" is the concordant observations of men who have examined that fact in different lights and from different points of view. The final aseptic statement of the textbook of taxonomy is a far cry from the experience of the discoverer so vividly described by A. R. Wallace. 
None but a naturalist can understand the intense excitement I experienced when at last I captured it [a new species of butterfly]. My heart began to beat violently, the blood rushed to my head, and I felt much more like fainting than I have done when in apprehension of immediate death. I had a headache the rest of the day, so great was the excitement produced by what will appear to most people a very inadequate cause.

Wallace was in fact a very sound observer, as well as co-discoverer of the modern theory of evolution. Yet we cannot but feel more secure knowing that the butterfly that so profoundly stirred him-as discovery always stirs the first discoverer-has also been seen and similarly described by others not so stirred.

Depersonalizing even fact, the diversity of individual commitment becomes all the more important when theory is concerned. The approach route of the innovator always leaves some marks on a newly created theory. It bears some imprint of his history, personal and scientific, and of his own cosmology and metaphysics. But, as noted in the last chapter, the theory will ultimately acquire its optimal form as it is used, and reconstructed, by others who do not share all its creator's preconceptions. Private knowledge, not wholly distinct from private inspiration, is thus winnowed, refined (and, on occasion, vulgarized) into the public science of the textbook. Dingle's testimony suggests the well-nigh incredible effectiveness of this process.

In contemplating the history of science one cannot but marvel at the sureness with which, by the instinct of the movement itself, the spurious has been eliminated and the genuine preserved. Kepler is roused to the same rhapsody by his theory of the five regular solids as by the laws that now bear his name; the first is forgotten, the second lie at the basis of dynamical astronomy. Doppler, . . . Balmer. . . . Over the terrain of rocks and quicksands science walks as sure-footed as a mule-and as sapient.

As in so many other cases, the "marvel of instinct" is the resultant of natural selection: here, just that natural selection of theoretic ideas described in the last chapter.

\section{PERTURBATIONS OF THE MECHANISM OF SELECTION}

In organic evolution the balance of stability and mutability is heavily weighted toward stability-lest the species peter out in a succession of stillbirths and monsters. As a whole the species "breeds true," but 
the entire possibility of evolution derives from the rare occurrence of abrupt mutations at the individual level. In science, too, the balance of stability and mutability is heavily weighted toward stability by the intellectual inertia of the vast majority of men. The forcefulness and spontaneity of individual genius is then more than counterpoised by the steady drag of organized science, the resistance of the great mass of scientifically "solid citizens." Their typical immediate response to fundamental novelty is accurately and charmingly reported by Arrhenius.

I came to my professor, Cleve, whom I admire very much, and I said, "I have a new theory of electrical conductivity as a cause of chemical reactions." He said, "That is very interesting," and then he said, "Good-bye."

We point to the Cleves, or the diehard opponents of Lavoisier, and deplore the stubborn inflexibility of uninspired opinion. The reprobation of scientific conservatism is, however, an artifact of the way we write the history of science. We make heroes of the insurgent champions of radical ideas whose insurgency was successful (e.g., Arrhenius and Lavoisier), and make knaves or fools of the conservative resisters of those innovations (e.g., Cleve and Priestley). But rarely if ever do we mention the bold insurgents who were wrong, and failed-they did not "make history." We have then as seldom occasion to celebrate the staunch resistance of their opponents, who maintained the predominance of conservatism over radicalism essential for an orderly evolution of science. Given a proper balance of these two factors, natural selection generally produces adoption of the correct (i.e., heuristically superior) ideas. However, on some occasions (all the more conspicuous for their comparative rarity) the mechanism has failed, usually for one or both of two reasons.

Defective personal commitment. Natural selection through the struggle of competing ideas will fail if the champions of new ideas fail to make a case for their acceptance. On rare occasions this failure seems to rise from an excess of personal commitment. Led by metaphysical speculations to which he was wholly committed, J. R. Mayer arrived at the conception of a conservation of energy. But, in thrall to his commitment (and emotionally somewhat unstable), Mayer was at first quite unable to separate his achievement from its inspira- 
tion-the scientific principle from the metaphysical matrix-and in consequence others who rejected his metaphysics wrote off his physics as well. Most scientists have succeeded better in making the required distinction. A somewhat more common miscarriage of natural selection arises from the innovator's deficient commitment.

Boyle questioned that the sandy deposit formed in water long boiled in glass vessels is actually transmuted water. But he did not think enough of his idea to carry out the experiments (with metal vessels, for example) he conceived would resolve the issue. Unsupported by action his scepticism counted for nothing, and we rightly regard Lavoisier as the man who actually solved the problem. Waterston created a kinetic theory years before its independent creation by others. Rebuffed by the publications committee of the Royal Society, Waterston did not make an effective fight for his ideas which, in consequence, had no effect whatever. Perhaps, for both geographic and temperamental reasons, he could not make such a fight: then resident in distant India, he was so cast down by his failure that he never undertook any other scientific work. In 1935 Noddack published her sceptical appraisal of Fermi's hypothesis of transuranic elements. But she did nothing to exploit, or secure the exploitation of, her idea that Fermi had produced an unprecedentedly gross nuclear transformation. Her idea then counted for nothing, and nuclear fission was discovered, quite independently, only in 1939.

Excessive authority. Presumably the intensity of personal commitment usually falls in the acceptable range where demonstrable deeds are produced if at all producible. But even then natural selection may fail. Natural selection must fail if excessive concentrations of authority make special sanctuary for certain species of opinion. Authoritarian perturbations of the mechanism of selection are most frequent wherever science overlaps the humanly compelling concerns of cosmology and technology. But even in pure science Gerhardt and Laurent could make little headway against the well entrenched enmity of a Dumas. High wisdom of the wielders of authority does not ensure wise exercise of authority. Planck's important doctoral dissertation failed to win the attention of any of the three great masters to whom he appealed-Clausius, Helmholtz, and Kirchhoff. Today the threat of authoritarian perturbation of natural selection is no doubt 
much reduced by the multiplicity of journals, research centers, and sources of support. In another respect, however, the threat is intensified: unfit ideas may well be "forced" to a false appearance of viability in the hothouse atmosphere of the great modern research factory headed by a crafty administrator, accomplished fund-raiser, and shrewd purchaser of others' talents. 

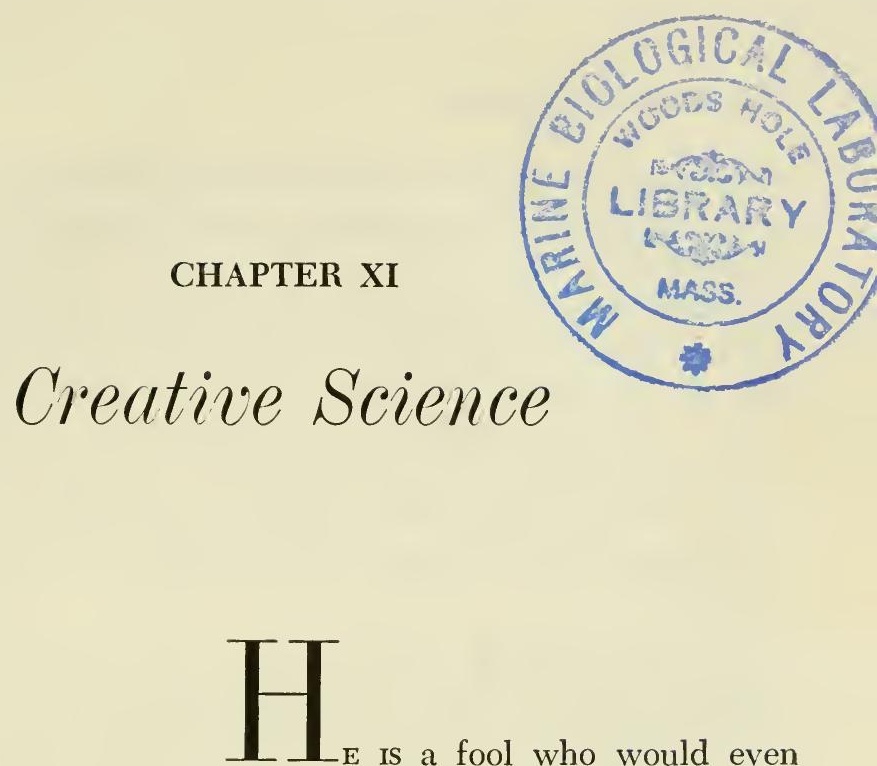

$\mathrm{E}$ Is a fool who would even attempt verbal description of the subtle skill of scientific research. Koestler lumps together a "high" and a "humble" skill in asserting that:

To ride a bicycle or play the violin requires great skill and "muscleknowledge"; but it is a knowledge which cannot be verbalized, and most of the perceptions of minute stimuli during the process, as well as the responses to them, take place on an unconscious level.

Even when skills involve no "muscle-knowledge" they still escape reduction to verbal formulae. Consider, as one somewhat special case, the path the hypothetical student of pulmonary radiology (see p. 14) must travel to gain mastery of that skill. Consider, as the most general possible case, what Bartlett adopts as the foundation postulate for his whole study (and book) on "Thinking."

Thinking has its acknowledged experts, like every other known form of skill, and in both cases much of the expertness, though never, perhaps, all of it, has to be acquired by well-informed practice.

Like the skill of thinking, the skill of scientific research involves an element of art that is not acquired when one has swallowed some encapsulated formula. Rather, that skill must be assimilated from the experience of research.

The skill that cannot be given the student can be acquired by him; 
what cannot be taught can be learned. And learning may be facilitated by imparting to the student various methodological maxims insufficient in themselves. Given the maxims, the student may more rapidly grasp the skill that escapes complete description in terms of maxims and, conversely, grasping the skill he sees at last the full import of the maxims. But surely what most effectively promotes the student's achievement of the skill will be close association with one who is master of it. "By watching the master and emulating his efforts in the presence of his example," says Polanyi, "the apprentice unconsciously picks up the rules of the art, ..." And elsewhere he remarks:

A master's daily labors will reveal these to the intelligent student and impart to him also some of the master's personal intuitions by which his work is guided. The way he chooses problems, selects a technique, reacts to new clues and to unforeseen difficulties, discusses other scientists' work, and keeps speculating all the time about a hundred possibilities which are never to materialize, may transmit a reflection at least of his essential visions. This is why so often great scientists follow great masters as apprentices. . .

\section{SCIENTIFIC METHOD: SHADOW AND SUBSTANCE}

We never credit the possibility of a definable Literary Method practice of which brings within reach of mediocrity the production of works of genius. Yet at least four reasons may encourage even master scientists, and others, to believe possible a communicable Scientific Method with some such magic power.

First: The striking progressivism of science may seem to find a plausible explanation in the idea that scientists systematically exploit some uniquely powerful Method. And the shape of Method is sketched long before science can itself lay claim to any special dynamism. For Bacon the door to scientific progress was to be unlocked by the same two-pronged key that had opened the way to progress in the practical arts: a thorough-going empiricism, coupled with a thorough-going disregard for both vulgar superstition and sophisticated but fossilized general systems of ideas. Method is thus powered by scepticism and centered on facts. In cursory reading of scientific history, if we always take for granted the availability of ideas, then progress will indeed seem to turn on the discovery of facts. Just so, if we take also for granted an unshakable confidence in certain be- 
liefs still unchallenged, progress will seem to result from successful challenges hurled by scepticism at once-authoritative beliefs now rejected.

Second: Much "literature" is comparatively routine hackwork that might not implausibly be thought produced by some formula of Literary Method. The vast bulk of scientific work-the necessary but generally uninspired exploitation of established conceptual systems -similarly gives color to a conception of Method. Here orderliness characterizes scientific advance, which simply fills out a theoretic pattern that directs the work of all. Here empiricism may seem dominant, for the primary concepts and hypotheses are already taken for granted. Here logic will seem self-sufficient: the great imaginative creation of basic views is already complete. Here detachment rules supreme: we can afford detachment when the points at issue are only details within a theoretic structure to which our commitment is so uniformly deep and pervasive as wholly to escape notice.

Third: Chronometric considerations may well suggest a concept of Method that makes no reference to hypotheses and imagination. Much the greater part of the scientist's time is devoted to preparations (the empirical collection of facts and the logical analysis of them) and to appraisals (logical analyses and the empirical confirmation of deductions drawn from them). Preparations for what, analyses of what? Nothing but the hypotheses and theories born of scientific imagination! However, a new idea may come in an apparently instantaneous flash, and the function of the scientific imagination may seem wholly spontaneous. The scientist may then quite understandably identify the essence of his practice with that at which he labors: the determinations of facts and the analyses of logic.

Fourth: To recommend his ideas to sceptical colleagues, the scientist must argue in terms of evidence-facts and logic. He may even suggest that his findings derive from, and depend on, nothing but facts and logic. Thus Newton, saying hypotheses non fingo, presents his theory as the fruit of logical induction; thus Darwin dons the mantle of the Baconian empiricist in expounding his theory of evolution. If any deceit is here involved, it is in no small part self-deceit. The scientist misidentifies the mode of exposition he finds most effective with the mode of discovery he used, or could (or should) have used. Another factor strongly promoting such misidentification is noted by Einstein: 
To the discoverer in the field of theoretical physics, the constructions of his imagination appear so necessary and so natural that he is apt to treat them as he hopes others will treat them: not as the creations of his thoughts, but as given realities.

Yet a third contributory factor is also active. The scientist loves science, and honors its "truths." He seeks public acknowledgment of these truths as supreme over the "idle fancies" of vulgar superstition, sophisticated obscurantism, and muddle-headed mysticism. He then wants to believe, and present, his truths as the gleanings of a superlatively reliable Method. Such will be a Method sceptical of fallible human ideas, a Method accepting the authority only of "indubitable fact" and "inexorable logic."

Reinforcing all such "reasons," and setting at nought all objections to Method, is the further potent factor suggested by Polanyi's shrewd observation that the scientist

. . . can accept . . . the most inadequate and misleading formulation of his own scientific principles without ever realizing what is being said, because he automatically supplements it by his tacit knowledge of what science really is, and thus makes the formulation ring true.

Thus it is, I think, that scientists can accept, and advocate, one Method while actually practicing quite another. The four terms stressed by Method (left column) represent the indicated four pairs of polar antitheses. One member of each pair, forever passing un-

Empiricism (and facts)

Scepticism (and detachment)

Logic

Order
Speculation (and hypotheses)

Faith (and commitment)

Imagination

Chance

mentioned, is always "understood." Both members of all pairs are duly represented in science, and it were folly to make of this a paradox or mystification. The scientist does not "reconcile irreconcilables." As with all human endeavors, science lives and functions in the midst of purely conceptual abstractions that correspond to nothing distinctly separable as such either in ourselves or in the world. We might then write of scientists in terms analogous to those used by 
Keynes in discoursing on what is required of the competent economist.

He must contemplate the particular in terms of the general, and touch abstract and concrete in the same flight of thought. He must study the present in the light of the past for the purposes of the future. . . . He must be purposeful and disinterested in a simultaneous mood; as aloof and incorruptible as an artist, yet sometimes as near the earth as a politician.

Order and chance are inseparables-and all the more obviously so in modern science, where many elements of macroscopic order are conceived resultant from microcosmic chance. In scientific history, too, long-term order incorporates, and is in part product of, "accidental discoveries" rendered meaningful by the context of order in which they occur. Logic and imagination form, in science, a second pair of inseparables. In the "inductive" production of theoretic postulates, the severely disciplined imagination of the scientist rarely if ever functions in complete disregard of logical considerations. Conversely, even deductive logic requires, as we saw, a "notion of appreciation" presumably demanding some function of the imagination; and a great deal of imagination may be required to draw a distinctly novel theoretic deduction.

When modern science was just dawning, the theorist Blaise Pascal wrote:

Ours is an incapacity for proof supreme over all dogmatism.

Ours is an idea of truth triumphant over all scepticism.

In the 19th century Claude Bernard exhorted his fellow empiricists to place themselves

. . . in an intellectual attitude which seems paradoxical but which, in my opinion, expresses the true spirit of an investigator. We must have robust faith and not believe.

How maintain such ambivalent mixtures of faith and scepticism? That question arises only from the misconception that makes antitheses of inseparable polarities. Faith and scepticism are no more than opposite faces of the same coin. Nowhere outside the asylum is there a wholly pure faith or a wholly pure scepticism. Scepticism of one authority is the measure of faith in another, and conversely. Consider Copernicus. He is sceptical of the everywhere-accepted 
Ptolemaic conception. The root of his scepticism? Faith, of coursefaith in the Pythagorean conception of a cosmos mathematically harmonious.

Consider now the last of our four pairs. At the price of doubly contravening the principle of polarity, we may conceive scientific knowledge as generated by the endless renewal of this cycle:

Concepts, hypotheses, and theories

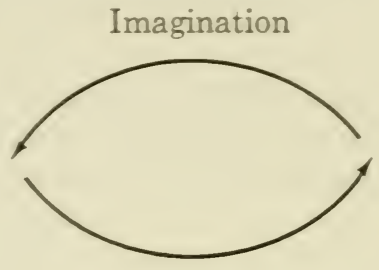

Logic

\author{
Facts, \\ observations, \\ and experiments
}

When the empirical results do not agree with what deduction from our hypotheses has led us to expect, we try to imagine some suitable alteration of our original body of ideas-and so initiate the first stage of the next generative cycle. The indissoluble connection of fact and hypothesis, already so much emphasized, is conceded at least implicitly by proponents of Method. But I wish now to argue, in direct opposition to Method, that primacy (if it attaches to either one of this pair) belongs far more to hypothesis, which first permits us to grasp fact, than it does to fact which may then suggest some further hypothesis.

The primacy of hypothesis. Within the context of aesthetics, Wölfflin remarks, "All pictures owe more to other pictures than they do to nature." Within the context of science, Darwin maintains, "Without the making of theories I am convinced there would be no observation." As for experiments, we saw in Chapter VI how they are conceived, designed, observed, and interpreted on the basis of prior hypotheses and theories. Now a companion insight of Darwin's further suggests that only certain experiments (or observations) are worth making.

How odd it is that anyone should not see that all observation must be for or against some view if it is to be of any service!

If "for" the theoretic view, it helps confirm it; if "against," it helps supply the impetus for the next generative cycle. But I think a rather 
more subtle point is also here involved. Is it not perhaps only those observations we can conceive as "for or against some view" that we will at all recognize as facts? Consider, for example, Bernard's appreciation of a significant episode in his own productive career.

without the original guiding hypothesis, the experimental fact which contradicted it would never have been perceived.

The reason we so generally overlook the primacy of hypothesis is because very often the hypothesis most concerned is not, like Bernard's, an explicit "guiding hypothesis." But it would seem that the primacy of hypothesis must still obtain even when it is not formulated as such at the outset. As a case in point consider that the crude technologic devices known to Galileo had many inadequacies. Of these one-the 31-foot limit on the action of lift pumps-particularly attracted his attention. Why? Galileo accepted the theory of horror cacui. Though he may never have formed the hypothesis explicitly: he doubtless supposed that the lifting capacity of a stoutly made pump would be limited only by the power available to drive it. $\mathrm{Be}$ cause it conflicted with this context of presupposition, the observed limit became an observation of interest, a fact worth looking at, "the trigger of inquiry" (Peirce)-a problem the solution of which yielded to Galileo's disciple. Torricelli, the new science of aerostatics.

The primacy of completely implicit hypotheses is a theme excellently developed by Whorf:

The familiar saying that the exception proves the rule contains a good deal of wisdom, though from the standpoint of formal logic it became an absurdity as soon as "prove" no longer meant "put on trial." The old saw began to be profound psychology from the time it ceased to have standing in logic. What it might well suggest to us today is that, if a rule has absolutely no exceptions, it is not recognized as a rule or as anything else; it is then part of the background of experience of which we tend to remain unconscious. Never having experienced anything in contrast to it, we cannot isolate it and formulate it as a rule until we so enlarge our experience and expand our base of reference that we encounter an interruption of its regularity. The situation is somewhat analogous to that of not missing the water until the well runs dry, . . .

We see now that the primacy of hypothesis can and must be maintained even as regards that important group of scientific events we 
call accidental discoveries. Accounts of such discoveries normally attach primacy to the observation of some startling fact. But why do we take notice of that particular fact, why are we startled? Precisely because the fact stands out from some "horizon of expectations" (Popper) set by prior hypotheses earlier perhaps completely implicit.

Important in itself, the primacy of hypothesis is important too for the serious problem it raises when the hypothesis is explicit and strongly held. Columbus, the very archetype of bold discoverer, vividly exemplifies that problem in a simple non-scientific context. Launched on his voyages by and with the passionate conviction that he could reach China and India by sailing to the West, Columbus never came to question that conviction. He never recognized that he had not reached China or India. Landed in the New World, he never recognized it as a New World and so, in a sense, failed fully to discover it. In science, too, we begin our observations and experiments with hypotheses and presuppositions to which we may be as deeply committed as was Columbus to his. How do we bring ourselves to recognize, often in situations of only marginal clarity, an appearance implying failure of a long-cherished inspiration, perhaps the trusted guide for a whole line of investigation?

\section{The Attitude of the Investigator}

Mythical Method enjoins an attitude of icy agnosticism also, as Polanyi suggests, strictly mythical.

A scientist must commit himself in respect to any important claim put forward within his field of knowledge. If he ignores the claim he does in fact imply that he believes it to be unfounded. If he takes notice of it, the time and attention which he diverts to its examination and the extent to which he takes account of it in guiding his own investigations are a measure of the likelihood he ascribes to its validity. Only if a claim lies totally outside his range of responsible interests can the scientist assume an attitude of completely impartial doubt toward it.

The only pure form of open-mindedness will then, appropriately enough, be found in finest flower only in the empty-minded who, we find, do not make scientific history. Indeed, the historical record shows some of the greatest scientists "lamentably" far indeed from 
the "ideal" attitude of passionless nihilism. The attitudes of such as Kepler, Galileo, Newton, Liebig, Pasteur, and Einstein are characterized far more by hot commitment than by cool detachment. Moreover, these men did not succeed despite their "unfortunate prejudices"-by those they were not simply guided but driven to labors otherwise wholly incredible.

Until we honor scientists for their inactivity, we will honor no scientist who has not been committed to his personal vision of reality, and so launched recklessly on action. The hazards of his commitment are borne in upon him as he sees others, perhaps almost all others, pursuing other visions through other actions. The hazards are still further compounded in that a sound vision may lead nowhere but to oblivion, while other ideas posterity will judge unsound may prove instruments to discoveries that establish claims to lasting fame. Given Kepler's successes, we honor his commitment. Had he failed, he would at most be recalled only as a competent Central European mathematician betrayed by his absurd Pythagorean faith that nature embodies discoverable mathematical harmonies. Perhaps it is less individual genius than history that makes the heroes. But nobody has ever become a hero of scientific history by shunning all faith because a case might be made for scepticism. Speaking of Kepler, Planck asks us to:

Compare him with Tycho Brahe. Brahe had the same material under his hands as Kepler, and even better opportunities, but he remained only a researcher, because he did not have the same faith in the existence of the eternal laws of creation. Brahe remained only a researcher; but Kepler was the creator of the new astronomy.

And his conclusion is this:

. . . science demands also the believing spirit. Anybody who has been seriously engaged in scientific work of any kind realizes that over the entrance to the gates of the temple of science are written the words: Ye must have faith. It is a quality which the scientist cannot dispense with.

In positivist circles faith is a fighting word, Planck a "relic of the old physics," and Kepler an atypical lunatic. Let us then come closer to home. The creation of the theory of special relativity is often cited as uniquely the fruit of positivist scepticism. Critical re-examination of the concepts and implicit assumptions of classical physics, by 
Mach and others, showed some of them unjustified by any experience of observables. And, so goes the fable, rejection of these unjustified concepts and assumptions then led "naturally" to a powerful new theory that, free from all metaphysical pretensions, sought only an economic description of observables. This account will not bear examination.

The scholastics' critical commentaries on the older physics paved the way for Galileo's reconstruction of mechanics and, to some extent, even for Copernicus' reconstruction of astronomy-both of which reconstructions were however rejected by the scholastics. The antecedent critiques of such as Mach cleared the way for Einstein-and Mach rejected Einstein's innovation. We never regard the works of Copernicus and Galileo as the triumphs of scholasticism; we have no better reason for regarding the work of Einstein as a triumph of positivist scepticism. However necessary as a preliminary to innovation, the critical re-examination of accepted theory does not itself produce innovation. If relativity is claimed for positivism we must at the very least set Mach's failure even to appreciate it beside Einstein's success in creating it.

Relativity theory is no trivial work of relativism but, on the contrary, fruition of the effort to grasp the invariant absolute within the context of observables always relative to observers. Not content to describe, Einstein sought to understand. And he had precisely what Mach lacked: that faith in the possibility of human understanding which so shines forth from a statement of his later years.

I still believe in the possibility of producing a model of reality-that is to say, a theory which will represent things themselves . . .

Reichenbach reports that:

When I, on a certain occasion, asked Professor Einstein how he found his theory of relativity, he answered that he found it because he was so strongly convinced of the harmony of the universe.

Had Reichenbach been on hand to interview Copernicus he would have received much the same answer: Einstein and Copernicus pursued parallel courses under the inspiration of the same faith. Reichenbach found Einstein's answer unsatisfactory-for "a creed is not a philosophy"-and others have held it absurdly metaphysical and scientifically immature. However that may be, the faith is nonetheless 
essential. The rebirth of the heliocentric system is, after all, the work of Copernicus-not of any Osiander who, with a reserve no positivist could fail to applaud, said of that system:

I have always felt about hypotheses that they are not articles of faith but the basis of computation; so that even if they are false it does not matter, provided that they reproduce exactly the phenomena of the motions.

\section{DETACHMENT}

Moved to action by commitment to our ideas, we can learn better ideas only as we bring some detachment to the scrutiny of the results of that action. The virtues of open-mindedness have been much extolled and, as Bernard emphasizes, they are not to be scorned.

Men who have excessive faith in their theories or ideas are not only ill prepared for making discoveries; they also make very poor observations.

On the basis of careful study of Mendel's report of his observations, Fisher concludes that Mendel must not infrequently have deceived himself, precisely as Bernard suggests. We may well deplore such self-deception, but we cannot simultaneously honor the work and condemn the faith that alone evoked and sustained it. What we must seek is some balance of commitment by detachment.

The institutional structure of science powerfully contributes to the attainment of such a balance: to some extent it even makes that attainment unnecessary, by opening the possibility of co-operative discovery. The New World that Columbus could not recognize is recognized by companions who more accurately read the "face value" of the evidence. Thus, in the scientific context, Hahn and Strassman do not themselves discover nuclear fission, but report the results of which they say:

As "nuclear chemists" having many close associations with physics, we cannot yet bring ourselves to make this leap in contradiction of all previous experience in nuclear physics. A series of strange accidents may, after all, have rendered our results deceptive.

Meitner and Frisch then go on fully to discover what Hahn and Strassman's reluctance to contradict nuclear theory kept them from recognizing. 
This possibility cannot fully solve our problem. Columbus is accompanied by others, but the scientist in his laboratory is not so accompanied. Very rarely will he report-if he "sees" them at all-observations that (given his presuppositions) he attributes to "faulty technique" or some "series of strange accidents." Editors and referees see only the individual's report of what he saw, and are powerless to determine what may have been overlooked or incorrectly seen. Thus this mechanism of co-operative discovery already presumes individual capacity to solve the central problem of recognition. Another possibility of co-operative discovery is suggested by the activity, in any given field of science, of many observers no two of whom share exactly the same presuppositions. One may then be able to recognize what none of his colleagues can "see." But the most fundamental presuppositions are likely to be shared by every investigator in a given field at a given time. For recognition of something out of keeping with these we are thus again thrown back to reliance on individual capacity to balance commitment by detachment.

What are the roots of individual detachment? The training and fellowship of the Invisible College unquestionably promote capacity for dispassionate observation, but this effect is clearly neither necessary nor sufficient. Even given this institutional support, many men have (like Mendel) failed to observe quite correctly. And, even lacking such support, a few men passionately committed to certain presuppositions have discovered in themselves the power to recognize appearances implying the inadequacy of those presuppositions. The paradigm of such a man is of course Kepler, who accepts an apparently trivial discrepancy as signifying the inadequacy of the presupposition of circular orbits earlier fully accredited by him as by everybody else.

Intelligence. Capacity to observe, as genuine, experiences contrary to expectation, is inhibited by the feeling of certainty that denies the possibility of such experience. The lively intelligence that can conceive alternatives to widely-held presuppositions will dissipate the unhealthy feeling of certainty, and correspondingly facilitate observation of what might otherwise be overlooked as "impossible." Even when intelligence does not supply alternate views at the outset, by producing them in the hour of need it may allow us to take cognizance of appearances otherwise so unwelcome as to risk going "unseen." Thus Beveridge comments that: 
The scientist who has a fertile mind and is rich in ideas does not find it so difficult to abandon one found to be unsatisfactory as does the man who has few. It is the latter who is most in danger of wasting time in hanging on to a notion after the facts warrant its discard. Zinsser picturesquely refers to people clinging to sterile ideas as resembling hens sitting on boiled eggs.

Such functioning of intelligence to produce detachment is of major importance, but does not fully solve our problem. Kepler and Ehrlich, for example, succeeded by clinging to ideas that most of their contemporaries held "boiled eggs"-ideas we might similarly dismiss had not Kepler and Ehrlich succeeded. Moreover, the very intelligence that helps produce detachment may blind us to the need for detachment. Thus Priestley's great ingenuity permits him to explain away all objections to the phlogiston theory suggested by Lavoisier's work. Here Priestley displays precisely that impairment of detachment by intelligence sketched in his own comment that

. . . we may take a maxim so strongly for granted, that the plainest evidence of sense will not entirely change, and often hardly modify our persuasions; and the more ingenious a man is, the more effectually he is entangled in his errors; his ingenuity only helping him to deceive himself, by evading the force of truth.

Detachment from commitment. The deepest source of detachment is commitment. This is no paradox: we see it often realized. As parents we address ourselves with passionate devotion to the rearing of a child. But from this very devotion arises in time a curious detachment. As responsible parents we recognize that some day our child must make his way alone in a difficult world. He must learn to do so, and we to let him learn. Because we love him, and are committed to faith in his potential competence, because we wish for him every success, we force ourselves to watch with detachment the often unsuccessful struggles by which alone he can at last achieve self-sufficiency.

Some parents are incapable of such detachment: they can never give up directing and protecting "for the child's good." Some scientists are similarly incapable of viewing their brainchildren with any degree of detachment. The chemist Thomas Thomson offers an apt example of this failing. He was convinced of the soundness of Prout's hypothesis, and instituted experiments to show that the atomic 
weights of all elements are conformable with it. In those early days of chemistry, procedures for the determination of atomic weights were still quite crude. Consequently, when Thomson's experiments yielded results incompatible with his hypothesis, he could easily find invalidating errors-some real, some imaginary. Making "appropriate" allowance for these, he then obtained "satisfactory" results, and sought no further for errors still present in abundance in his experiments. So proceeding, he simply contrived to find what he had expected to find: his experiments taught him nothing, nor was the hypothesis he espoused in any way strengthened. Berzelius' appraisal of this work is harsh but not unjust.

This investigation belongs to that very small class from which science can derive no advantage whatever; . . . and the greatest consideration which contemporaries can show to the author is to treat his book as if it had never appeared.

Compare with Thomson's the behavior of one we deem an eminently successful parent of scientific brainchildren. Nobody could have shown a more passionate commitment to his ideas than Louis Pasteur. Yet his experiments are soundly observed, are great mines of new knowledge. Out of the depth of his commitment to ideas which he felt must survive all tests, Pasteur gathered the detachment required to put those ideas to the most searching tests he could devise. He thus lived in fact by the advice he offered others.

It is indeed a hard task, when you believe you have found an important scientific fact and are feverishly anxious to publish it, to constrain yourself for days, weeks, years sometimes; to fight with yourself, to try and ruin your own experiments and only to proclaim your discovery after having exhausted all contrary hypotheses.

A brainchild so tried may be deemed competent to make its own way in the world.

A temperamental factor is deeply involved in such a birth of detachment from commitment. Indeed, the most extreme detachment will be born from the most extreme commitment of the man of strong self-confidence-commitment to his own competence to deal with anything he may encounter. In examining his experiments such a man feels no need to put on blinders, because there is nothing he is afraid to see. He feels no need to shield his preconceived ideas, because he is confident that as required he can produce new and better 
ideas. One possessor of such a temperament comes vividly to life in Richet's sketch:

Magendie was delighted when experiment gave a result contrary to what he was expecting. "Well!" he used to say, "I was mistaken, but it is much more interesting than if I had succeeded. I foresaw, as probable, a logical orthodox fact, which everybody might expect; and it is just the contrary that happens. There is therefore a new phenomenon, so much the more important as it was so little expected." And Magendie did not hide his satisfaction at having run aground.

A Magendie can then view with detachment-nay, with positive enthusiasm-the failure of the presuppositions that inspired his work. Is this the route, open perhaps only to Magendies, by which one arrives at that openness to experience that $\mathrm{C}$. $\mathrm{R}$. Rogers considers characteristic of the creative individual?

... instead of perceiving in predetermined categories ("trees are green," "college education is good," "modern art is silly") the individual is aware of this existential moment as $i t$ is, thus being alive to many experiences which fall outside the usual categories (this tree is lavender; this college education is damaging; this modern sculpture has a powerful effect on me).

However produced, this openness to experience is a critical determinant of the scientist's capacity to make what we call "accidental discoveries."

\section{Accidental Discoveries}

We regard as accidental those experimental discoveries that, in the absence of any preconception of what was to be found, were less sought than stumbled upon. Such discoveries fall in two classes. Focal discoveries involve observation of some unanticipated occurrence within the area of primary attention. A very much smaller group of peripheral discoveries involve recognition of occurrences quite outside that area, over the horizon of expectation.

\section{FOCAL DISCOVERIES}

Perhaps as accidental as any discovery could well be is Malus' detection of the polarizability of light. Apparently quite idly, he happened to look through a doubly refractive crystal at the late after- 
noon sunlight reflected from the windows of the Luxembourg Palace. He observed a totally unexpected variation of the intensity of the transmitted light with the orientation of the crystal held in his hand. The role of chance here is enormous: for example, had the angle Malus-ivindow-sun been much different, there would have been no effect for him to observe. On the other hand, in the happy circumstances which did exist this was a focal discovery: it manifested itself in the light to which Malus' eye was directed.

Priestley prepared gases by heating strongly various materials selected more or less at random. Given chemical thought of the time, Priestley examined each gas specimen for certain "qualities"-of which ability to support combustion was one. A great display of such ability in one particular gas specimen ultimately led Priestley to the discovery of oxygen. The original discovery was, as Priestley himself regarded it, quite accidental. But, the quality concerned lying within the purview of attention, it plainly was a focal discovery.

Roentgen accidentally discovered X-rays by the fluorescence they produce. Though wholly unanticipated, the discovery was focal. Roentgen was using a fluorescent screen as a radiation detector, and what he noticed was that the screen fluoresced when no familiar radiation was reaching it. Becquerel's discovery of radioactivity is entirely similar. While using photographic plates to detect supposed $\mathrm{X}$-rays emitted by uranium compounds, Becquerel found one plate very heavily blackened when he had not expected it to be so. Rayleigh finds the first clue to the discovery of an unthought-of element, in a minute discrepancy in the densities of samples of "pure" nitrogen. But these densities were, of course, the direct focus of Rayleigh's attention.

May it be that focal discoveries present no problem? After all, the unexpected occurrence manifests itself as a surprising value of some quality which is already under direct examination. How can one then possibly fail to observe the phenomenon? So stated, the question denies the problem. For the whole difficulty of accidental discovery is just the recognition that a surprising manifestation is a phenomenon. Examples of focal discoveries that were missed testify how very real that difficulty is.

Mitscherlich, skillful chemical crystallographer, examines racemic acid with a view to describing its crystal habit. The shape of these crystals is the focus of his attention, but he saw nothing whatever 
of a vital detail later observed by Pasteur. Lalande, thoroughly competent astronomer, plots the position of the stars. Two observations of the same star fail to agree. To us, knowing of the existence of Neptune, the discrepancy indicates a phenomenon. To Lalande it indicated only an observational error, and he threw away the opportunity to discover a new planet. Rayleigh published a note calling attention to a small but puzzling disagreement he had observed in the densities of gaseous nitrogen. Not one of the hundreds of scientists who read the report of this celebrated physicist saw in it anything but a trivial discrepancy. Hillebrand, meticulous analytical chemist, observed the spectrum of "nitrogen" extracted from clevite, and noted certain lines he was unable to associate with those of any element known to him. He dismissed the strange lines as some peculiar artifact of the conditions under which the discharge was excited.

Apparently it is all too easy to see not a phenomenon but, with Mitscherlich, nothing at all; with Lalande, an observational error; with Rayleigh's contemporaries, a trivial discrepancy; or with Hillebrand, an artifact of the apparatus. By such expedients the unexpected manifestation is, if at all recognized, dismissed not as a phenomenon but as an irritation. Accidental discoveries which are focal discoveries can be missed, have been missed, by scientists of high competence. And we cannot simply say that those who did not miss them are a "lucky" few of many whom history might equally have made heroes. The "luck" is too unevenly distributed: the vast majority of scientists have none, while "happy accidents" seem constantly the fortune of such as Priestley, Faraday, and Bernard. Whence derives their serendipity?

\section{THE THRESHOLD OF IMPRESSIONABILITY}

I read a book that interests me. All around me are sources of sounds that set my eardrums into a haze of vibrations. But most of these auditory stimuli are dismissed without ever rising to full awareness-e.g., the children bouncing a ball, the dog scratching at the door, my neighbor mowing his lawn. In order to "pay attention" to those few (among many) sensory stimuli that matter, I have depended on an automatic screening mechanism that excludes from consciousness myriad "distractions." Even within the focus of my attention I will have failed to notice some things, e.g., most misprints or editorial inconsistencies. 
I have a threshold of impressionability (hereafter, TI) that most stimuli do not surmount. Two factors are involved: the "subjective" setting of the threshold value and the "objective" nature and magnitude of the stimulus. Reflecting the first factor, some people can concentrate in the midst of distractions which deprive others of all possibility of fixing their attention. Reflecting the second factor, a loud or abrupt noise breaks in on my attention when a softer or more sustained noise does not. But what matters most is neither factor by itself, but rather the extent to which the stimuli do or do not fit what I accept tacitly as the pattern of normality or non-significance. The waxing and waning roar of my neighbor's mower makes no impression on me, though I would become aware of the faint scratching at the door were I not the owner of a dog.

The scientist in his laboratory has also a threshold of impressionability. In any experiment some things can be observed only at the price of willingness not to "see" a multitude of others. The horizon of expectation defines the focus of the scientist's attention. To events beyond that horizon he gives little or no consideration, which is of course why the peripheral class of accidental discoveries is so small. But even within the focal area not everything will be noticed. The scientist's training and experience teach him that in all experiments there are apt to be minor variations arising from assignable and unassignable fluctuations in the experimental conditions. To be recognized even as a discrepancy a focal datum must surpass some vague margin of reasonable variation. This point is well displayed in the very general scientific problem of identification.

Consider how Priestley came to discover "dephlogisticated air"what we call oxygen. First comes the focal discovery that the gas from mercurius calcinatus per se is a brilliant supporter of combustion. The effect is large and striking, and immediately put Priestley in mind of a "factitious air" he had earlier found to behave similarly. The property was, as far as Priestley knew, unique. He had then no hesitation in (mis)identifying the new gas as what we call "laughing gas." Actually there are perceptible differences in the appearance of a candle burning in oxygen and one burning in laughing gas: in oxygen the combustion is somewhat more brilliant, and in laughing gas some brownish vapors are produced. The differences are noticeable, but they were not at first noticed by Priestley. These combustion phenomena are not highly reproducible in experiments as crude as 
Priestley's were. He was then prepared to find some variability in the appearances; and so, understandably, failed to observe the small differences in the presence of the far more obvious, and presumably significant, similarity.

Priestley's discovery of the inadequacy of his first identification is also typical: a tacit expectation founded on this hypothesis broke down by a wide margin. Here the discovery is peripheral. Priestley noticed that during storage over water a specimen of the new gas remained substantially unchanged in volume after a period in which laughing gas would have been largely if not completely dissolved. That is, Priestley noticed (though this lay well beyond the focus of his attention) that the new gas is much less soluble than laughing gas. The TI surmounted, Priestley turned back to repeat the combustion experiments. And now, having reason to look more closely, he did notice the small differences within the overriding similarity. The new gas is not laughing gas.

Priestley here emerged only to plunge right back into error. $\mathrm{He}$ applied his nitrous air test to the new gas and saw that-like ordinary air-it gives a substantial volume contraction and a copious production of brown fumes. The similarity is overwhelming, and he had then no doubt about his second (mis)identification of the new gas as common air. The proportionate volume contractions are noticeably different; but the difference does not much exceed the intrinsic variability of the test, and so was not noticed. This kind of error is not peculiar to Priestley: the identical error was made in the identical way by the celebrated Lavoisier. Lavoisier indeed never detected the error until it was pointed out to him by Priestley, who had by then again encountered, here as a focal discrepancy, a major breakdown of an expectation founded on the prior identification. In a random experiment he found that the residue left from the nitrous air test of the new gas still supported combustion brilliantly. But when ordinary air is tested the residue, far from supporting combustion, extinguishes a flame. The discrepancy is immense, the TI is exceeded, and Priestley returned to a more careful examination of the nitrous air tests. And then at last he noticed the small differences and, ultimately, identified his material as a new species of gas.

This ancient story deserves review only because it is representative of so many other like instances in the history of science. The problem of identification is, of course, still with us-observe the de- 
lay in the discovery of nuclear fission consequent to the misidentification of fission products as transuranic elements. Even when the discrepancy is large enough to be noticed, it is most likely to be at once dismissed from thought as experimental error. Polanyi emphasizes that, since the vast majority of discrepancies do not signify new phenomena, such dismissal is generally the correct policy.

In my laboratory I find the laws of nature formally contradicted at every hour, but I explain this away by the assumption of experimental error. I know that this may cause me one day to explain away a fundamentally new phenomenon and to miss a great discovery. Such things have often happened in the history of science. Yet I shall continue to explain away my odd results, for if every anomaly observed in my laboratory were taken at its face value, research would instantly degenerate into a wild-goose chase after imaginary fundamental novelties.

Conant suggests that a recognizable discrepancy is most likely to be accepted as a significant discrepancy only when it far exceeds the investigator's own estimate of probable experimental error. Take the case of Rayleigh, who noticed and reported a discrepancy of $0.1 \%$ in his measurements of the density of nitrogen. By the standard of ordinary gas-density measurements this was a trivial discrepancy, well within the zone of experimental uncertainty, and it was dismissed as such by those who read Rayleigh's report. But to Rayleigh, who had confidence in his work, this discrepancy far exceeded any reasonable estimate of experimental error, and he pursued the matter to discover a genuine phenomenon-the occurrence in the atmosphere of the new element argon.

In some cases the effect observed is prodigious both absolutely and relatively. But, as is plain in the case of Rayleigh's work, all that matters is the magnitude of the anomalous effect relative to the setting of the TI. This setting varies with the state of the science concerned. It is apt to be very high in areas already exhaustively explored: in the area of the physics of simple mechanical assemblies the TI is today almost indefinitely high. In certain recently opened areas in biochemistry it is very low indeed. But, even in a given scientific area at a given time, there will be enormous variation in the TI from one scientist to another. All workers will recognize the need for discrimination in setting the TI neither too high nor too low. But sound discrimination in this sense can be seen in retrospect as one of the most distinctive marks of individual genius. Polanyi remarks an apt example: 
Rutherford's genius has been well characterized in this connexion by one [C. G. Darwin] who knew him closely. He could throw aside as irrelevant a stream of reports pouring in from all over the world about new oddities to which fellow scientists called his attention, and yet respond to one particular instance among them, raising a hue and cry such as caused [his colleague and former student] Chadwick to discover the neutron.

What factors determine the individual's setting of the TI?

Knowledge and expectation. Even to recognize a discrepancy as such, prior knowledge is required. One must know enough of the theories and laws of the science concerned to anticipate what should happen; and one must know enough of its experimental procedures to estimate how closely that anticipation should be borne out. Some more specific knowledge may also be required to recognize a phenomenon in some deviation from the base line of expectation. Hillebrand, no expert in spectroscopy, saw nothing in the spectrum of the gas from clevite but a failure to correspond exactly to the spectrum of nitrogen-and he set this failure down as an artifact of the experiment. But Crookes, a highly experienced spectroscopist, no sooner set eye to eyepiece than he saw that several of the spectral lines were identical with some earlier attributed to a hypothetical solar element, helium. Given his prior knowledge Crookes saw more than an artifact, more even than a discrepancy. He saw a phenomenon: the terrestrial occurrence of helium.

Whether or not we call Crookes' discovery accidental is primarily a matter of definition. Certainly he had no anticipation that he was to find helium. But, unlike Hillebrand, he was prepared to find something more than nitrogen: he thought argon might be present. Thus he brought to the examination of the spectrum a different "set": he was less interested in the resemblance of the spectrum to that of nitrogen than in any perceptible difference between the two spectra. For any such difference(s) the TI was set low and, a difference being noted, Crookes' past experience at once made it a phenomenon.

Pasteur writes:

In the fields of observation, chance favors only the mind which is prepared.

Even better than the Crookes episode, Pasteur's own work illustrates again and again the value of such preparation. Thus, for example, 
Mitscherlich's report that the crystals of tartaric and racemic acids are identical in structure violated a law of hemihedral correlation credited by Pasteur, and so disturbed him that he undertook to repeat Mitscherlich's work. Pasteur could have had no expectation of what he was to find. But he was "set" to look for differences where Mitscherlich had found only identity, and his attention was more narrowly focused than Mitscherlich's-on the hemihedral facets rather than on the crystals as a whole. Most of all, Pasteur was prepared to recognize what was presented to his eyes, as to Mitscherlich's, because he could at once grasp the meaning of what he observed.

Intelligence and temperament. When prior anticipatory ideas point to some specific possible novelty, that novelty is not discovered accidentally. But, though strong enough markedly to depress the TI, Pasteur's expectation of novelty was here wholly formless-and chance still played a substantial role in his discovery: e.g., very few racemic mixtures are spontaneously resolved as this one happened to be. Whether or not we deem Pasteur's discovery accidental is really quite irrelevant. The crucial factor in his success is precisely that suggested by Whewell's comment that:

No scientific discovery can, with any justice, be considered due to accident. . . . facts cannot be observed as Facts except in virtue of the Conceptions which the observer himself unconsciously supplies; . . .

Just so Beveridge comments that "Noticing . . . is mainly a mental process"; and Bernard writes, of a missed opportunity in his own illustrious career, that:

. . . we had the fact under our eyes and did not see it because it conveyed nothing to our mind.

Pasteur did not miss his opportunity. Confronting what had before confronted Mitscherlich, he noticed what had before been unseen because he could produce the idea that made a phenomenon where before there had been none.

Intelligence can thus teach the scientist how a discrepancy may be a phenomenon. Conversely, grasping that possibility, he may come fully to recognize a discrepancy of which he was earlier only marginally aware. We have all had personal experience of the "double 
take." All of a sudden, with an abrupt local lowering of the TI, we turn back to look again, at something originally dismissed without much thought, as we grasp what it may signify. We "get it," and are the more apt to do so when-to prior knowledge and an intelligence prolific of new ideas-we join that openness to experience earlier noted. Important even as regards focal discoveries, this temperamental trait is absolutely crucial in the making of peripheral discoveries.

\section{PERIPHERAL DISCOVERIES}

To notice any manifestation beyond the focal region is exceedingly difficult. Just how difficult is suggested by both the rarity of peripheral discoveries made and the abundance of peripheral discoveries missed. Consider two celebrated misses. Crookes, meticulous observer who found in an evanescent spectral line the clue to the discovery of the new element thallium, worked much with high vacuum discharge tubes. He noticed that unexposed photographic plates became fogged when stored, in their wrappers, in the vicinity of his discharge tubes. Crookes acted with exemplary promptness: he filed a complaint with the manufacturers of his plates-and so left for Roentgen the later focal discovery of X-rays. Second case: many investigators (not least among them Tyndall and Pasteur himself) observed that bacterial cultures, made for this or that purpose, failed to grow properly in the presence of such foreign contaminants as molds. The consequent determined effort, to improve culturing techniques, facilitated exclusion of the annoying contaminants-and so left to Fleming the discovery of penicillin.

An empiricist sceptical of the completeness of existing theoretic ideas, the successful peripheral discoverer believes that there is far more to be seen in nature than has yet been found. He stands in sharp contrast with the many who-taking scientific theories as finaldepend on those theories to dictate the roster of "significant" qualities and categories that alone are worth considering. Thus Bruner and his colleagues comment that:

. . . in attempting to differentiate exemplars from nonexemplars of a category, as one so frequently must in science, medicine, and indeed in daily life, the person will, in the absence of other information, tend to fall back on cues that in the past have seemed useful, whether these cues have been useful in an analogous situation or not. 
Rejecting the finality of the accepted set of cues, the maker of peripheral discoveries is capable of giving his attention even to those over-the-horizon clues to something that, were current knowledge supposed final, simply cannot exist. His attention is more mobile because he forever expects the unexpected. Hooke suggests that

... the believing strange things possible may pernaps be an occasion of taking notice of such things as another would pass by without regard. . .

Bernard praised always a "philosophic doubt," but he never doubted certain fundamental credos of the peripheral discoverer.

If we find disconcerting or even contradictory results in performing an experiment, we must never acknowledge exceptions or contradictions as real. That would be unscientific. We must simply and necessarily decide that conditions in the phenomena are different, whether or not we can explain them at the time.

. . . what we now call an exception is a phenomenon, one or more of whose conditions are unknown; . . .

Nothing is accidental, and what seems to us accident is only an unknown fact whose explanation may furnish the occasion for a more or less important discovery.

So believing, the man made for discovery becomes able to see peripheral manifestations others miss-and all the more discerningly when he is endowed with wit enough to conceive the something those manifestations might signify. The twitching of a frog during dissection is not so much a puzzling as an absurd triviality of which Swammerdam made nothing. But Galvani conceived the twitching a phenomenon, associated with the operation nearby of an electrostatic generator. Just so the regression of bacterial colonies first became a phenomenon when observed by Fleming. His mind prepared by long labor to contrive the ideal antiseptic he thought possible, Fleming conceived that the mold secretes a bacteriocidal agent. The accidental discovery is consummated in the conceptual invention of the idea of a phenomenon-and this, no work of empiricism or logic, is the fruit of a creative imagination of which Bernard says most of what can be said:

A propos of a given observation, no rules can be given for bringing to birth in the brain a correct and fertile idea that may be a sort of in- 
tuitive anticipation of successful research. The idea once set forth, we can only explain how to submit it to the definite precepts and precise rules of logic from which no experimenter may depart; but its appearance is wholly spontaneous, and its nature is wholly individual. A particular feeling, a quid proprium constitutes the originality, the inventiveness, or the genius of each man.

\section{The Creative Imagination}

I cannot say what imagination is, nor can I assign the causes of imagination. As regards science, I suppose it possible to say what imagination does, and to indicate some of the factors influencing its function. We exercise imagination when we form our experience in new patterns we have not been taught, nor taught to expect. This capacity we find in children. Their fantasies sometimes amuse, sometimes horrify, but they do represent novel groupings of experience. While awake we ourselves can rarely conceive the world in such "fantastic" ways. Yet, when freed from the controls of consciousness, even adult imagination will again reconstruct the world. Our dreams, like the fantasies of childhood, present novel groupings of facts and ideas. They involve new combinations and separations, and new attributions of importance and unimportance, of naturalness and unnaturalness-and so do the great breakthroughs of scientific imagination.

Education. We cannot be taught imagination, but education cannot fail both to enlarge and to contract the potential imaginative capacity of which each has his own native endowment. Education enlarges imagination by conveying knowledge of multiple facts and relations, and of the many conceptual patterns in which various groups of these can be arranged. The educated man is then better equipped for the creation of novel patterns: he knows what there is to be fitted into them, and he is able to frame them by analogy with patterns he has been taught. On the other hand, education contracts imaginative capacity to the extent that it suggests that only certain patterns are permissible or "right." In this respect some educations will be worse than others but, by the very nature of the situation, some such contraction seems unavoidable. Even as we learn our language we are constrained toward certain conceptual patterns, and our capacity to conceive patterns not conformable with the linguistic categories may be seriously impaired. 
The child's free-ranging imagination is of no use to science. Knowing too little and accepting too much, he fails to discriminate between imaginative constructions that apply to things as they are, or might be, from other constructions that don't. The educated man has acquired this vital power of critical appraisal, but often he pays for it an inordinately heavy price. Certain thought patterns (if formed at all) may never rise to his consciousness, because they are at once swept aside by an act of appraisal that overlaps the act of imaginative creation. There is a selective inhibition of patterns "not worth thinking about"-and, alas, some of the suppressed patterns may have potential value. Too severely chastened by education, "responsible" imagination may yield only stereotypes. Indeed a difficult feat, apparently rarely accomplished, is the acquisition of knowledge and discipline without loss of essentially all of the child's facile capacity for seeing the familiar in unfamiliar ways. "Genius is youth recaptured," says Baudelaire in one context; and in quite another Cohen suggests that:

The essence of scientific genius (whether in the natural or the social sciences) is just this ability to discover points of view from which new arrangements of facts are visible. . .

Youth. There is considerable evidence for belief that scientists generally do their most important work by the time they are 35 . The recent record is indeed striking, but comparatively young men seem responsible for most major theoretical innovations in all sciences in all times. This would not unreasonably be the case. Imagination is shackled by the habits of a lifetime spent in use of some particular conceptual pattern $(s)$. Beveridge remarks the occurrence of an analogous situation on a much shorter time scale.

Psychologists have observed that once we have made an error, as for example in adding up a column of figures, we have a tendency to repeat it again and again. . . . The same thing happens when we ponder over a problem; each time our thoughts take a certain course, the more likely is that course to be followed the next time. Associations form between the ideas in the chain of thoughts and become firmer each time they are used, until finally the connections are so well established that the chain is very difficult to break. Thinking becomes conditioned just as conditioned reflexes are formed. We may have enough data to arrive at a solution to the problem, but, once we have 
adopted an unprofitable line of thought, the oftener we pursue it, the harder it is for us to adopt instead the profitable line. As Nicolle says, "The longer you are in the presence of a difficulty, the less likely you are to solve it."

Rather more than inability to meet a difficulty may be involved: there may be inability to see a difficulty that habitually accepted patterns of thought deny. Youth can thus be doubly privileged in confrontation of problems others have grown gray in seeking to solve, or to ignore. And, in characteristic challenge of what its elders have held unquestionable, youth may detect the implicit assumption that has concealed a problem where there is one-or made a problem where there is none: Alexander was a young man when he cut the Gordian knot.

Strongly favoring productiveness of new conceptual patterns, youth is not absolutely essential to such creativity. Older scientists may remain highly productive of major new ideas. Yet I find comparatively few who, like Planck, have in maturity first initiated any great work of theoretic re-construction. The vast majority of older innovators in ideas seem to fall in three other categories that do not at all infringe on the privileged status I have imputed to youth. There is first the group of those who make important extensions or refinements of existing theoretic patterns, without making any fundamental re-construction thereof. Perhaps this is where Dalton fits. And here surely we will place the great empiricists, such as Roentgen, who interpret in familiar terms the strikingly unfamiliar phenomena they discover in their later years.

A second group comprises those who in maturity completed and declared a major theoretic re-construction they actually initiated at a much earlier period. These are the great men described by Comte:

What is the life of a great man? It is a thought of youth realized in maturity.

Jenner took thirty years to bring to a test his youthful conception that cowpox might confer immunity to smallpox. Newton and Einstein recognized in their teens the problems they fully worked out years later. Copernicus and perhaps also Galileo belong in this category.

The third group of older innovators contains those who in maturity passed from one scientific field to another whose problems they could see with something of the freshness of youth. In scientific research 
one then encounters the situation Butterfield sketches in historical research:

Sections of history are liable to be transformed- . . . by an imagination that comes, sweeping like a searchlight, from outside the historical profession itself. . . . New matter emerges because things are joined together which it had not occurred to one to see in juxtaposition. New details are elicited, difficult details become relevant, because of a fresh turn that the argument has taken.

When an "invader" moves into another scientific field he brings with him more than a fresh insight. In his mind are joined two ordinarily separate bodies of knowledge, and the formation of a novel conceptual pattern may then be immensely facilitated.

Tuo fields of view. All scientists have at least two fields of view: the world of their particular science and the world of common sense. Many know something of the abstract world of mathematics, some are familiar with parts of the world of technology, a few join with their own science other bodies of special knowledge. Copernicus' professional concern was not astronomy but ecclesiastic law and administration; Lavoisier was a businessman, Dalton a meteorologist, and Kekulé a student of architecture before they turned to chemistry. Pasteur was a crystallographer before he became a bacteriologist, Crick a physicist before he became interested in biochemistry, and Mendel brought to his work what Zirkle identifies as an unusual combination of knowledge and experience:

Mendelism was the creation of an investigator who hybridized plants and who also raised bees.

An uncommon combination of fields of view, like youth not essential, like youth powerfully promotes creation of novel conceptual patterns. Most obviously, one may thus be led to apply in a new area a conceptual technique earlier developed in another. A more important possibility is the joining together of groups of facts normally distributed among different sciences. These are separated by boundaries that, partly matters of historical accident, are in part also reflection of ancient and current patterns of thought. The formation of a new conceptual pattern may then be possible only for him familiar with the two or more distinct disciplines that deal with the facts whose mutual relevance he will be the first to show. 
Use of the conceptual pattern of one field as model or analogue for another is a third and still more important possibility open to the fortunate possessor of multiple fields of view. Thus both Darwin and Wallace, independently, derived from Malthus' sociologic concept, of a struggle for survival, the mechanism of biological natural selection on which they founded the theory of evolution. In maturity, McGeoch maintains, transfer is a primary factor in all learning.

Where the subject "sees into" the fundamental relations of a problem or has insight, transfer seems to be a major contributing condition. It is, likewise, a basic factor in originality, the original and creative person having, among other things, unusual sensitivity to the applicability of the already known to new problem situations.

Transference of a pattern imposes a particular organization on what may earlier have been an inchoate mass of data. And, given the new questions that will be suggested by the model or analogy, further investigation acquires a definiteness of direction earlier perhaps notably absent. One may justifiably generalize to other sciences Duhem's comment that:

The history of physics shows us that the search for analogies between two distinct categories of phenomena has perhaps been the surest and most fruitful method of all the procedures put in play in the construction of physical theories.

The situation. A merely negative sense of dissatisfaction with an existing conceptual pattern can, in many ways, contribute positively to the creation of a new pattern. For one who finds the older pattern repugnant (as Copernicus found the Ptolemaic system), the fabric of the phenomena and relations woven together in it is loosened, and the contents become potentially available for reweaving in a new pattern. Moreover, efforts to "save" the older theory may produce a suggestive clustering of difficulties and ad hoc postulates which become, as we saw earlier, the focus of the effort to create a new theory. If, for example, a "subtle fluid" (e.g., ether, caloric, neutrino) has been made to function as solvent for anomalies, evaporation over the fires of scepticism may lead to recovery of the anomalies in partially crystalline form-already partly restructured in a new pattern. Even when this situation does not obtain, innovation is encouraged by the continued failure of (or continued complications enforced by) the efforts to "save" the older theory. Thus convinced that there is no 
easy way out, the potential innovator may be emboldened to question the indubitable, and to consider seriously the otherwise absurd. In just this way, Copernicus was brought to consider the hypothesis of a moving earth.

Julian Huxley argues that some Lamarckian appearance to evolution by natural selection is readily understandable. As, in the struggle for survival, an organism strains to the limit some capacity it already possesses, it finds itself ideally placed "to take advantage of" a mutational extension of that capacity-which might not otherwise be fully used, and so genetically "fixed." The straining of the capacity does not produce the mutation; the straining of an older theoretic pattern does not itself produce a new one. A further parallelism of much deeper significance is suggested by Weizsäcker's comment that

. . . even if someone came today who knew the answer to all unsolved problems, we should not understand him if our own need had not already driven us to put the questions which he answers. No help comes where a need has not even been felt.

The feeling of such a need produces in the potential innovator a general lowering of the TI-an enhanced receptivity to novelty, both conceptual and empirical. The gun so primed by uneasiness, a trigger discovery may now release the conceptual explosion in which the older pattern of thought is abruptly restructured. De Broglie provides a vivid sketch of the entire development as it is experienced by "the inventive theoretician."

No longer does he dismiss the gaps in previous theories as mere anomalies that will disappear when they are correctly fitted into the field as a whole, but rather as a shortcoming of the theories themselves. His keen interest aroused, he becomes aware of a mass of small and apparently unconnected facts and he begins to suspect hidden relationships that can only be explained by a theory based on entirely new ideas. Thus a geologist, surveying a vast region formed by recent alluvial deposits, and noticing the emergence of occasional outcrops of granite, may suddenly suspect that here has emerged a deep layer of ancient formations, once the shelf of the entire region, and explaining its structure. Thus small facts that were apparently pure accidents or anomalies suddenly appear as external signs of a previously unrecognized but fundamental unity.

In perception we make a figure-ground distinction. In writing history we distinguish a "scientific revolution" from the build-up of the 
conditions necessary for revolution. But the not uncommon phenomenon of simultaneous discovery alerts us to the importance of this build-up. The most subtle of the preparatory developments is probably a very gradual refashioning of scientific language, in forms that facilitate conceptual reconstruction. Thus Polanyi maintains that:

. . . every use of language to describe experience in a changing world applies language to a somewhat unprecedented instance of its subject matter, and thus somewhat modifies both the meaning of language and the structure of our conceptual framework. . . . the reiteration of linguistic utterances with reference to identifiable occasions carries with it a change of their meaning, . . .

Far beyond this, Tyndall remarks that the potential innovator will also find, already afloat in the intellectual atmosphere of his time, more or less vague intimations of the ideas that will determine the form of the new conceptual pattern.

Before any great scientific principle receives distinct enunciation by individuals, it dwells more or less clearly in the general scientific mind. The intellectual plateau is already high, and our discoverers are those who, like peaks above the plateau, rise a little above the general level of thought at the time.

The man. Of many reared in the same culture, comparably educated, equally youthful, with much the same fields of view, living in the same scientific situation-how very few rise at all above the plateau. The active variables are not yet exhausted, nor likely soon to be so. The flight of a human's thought is often inertial: small details of individual history may thus ultimately produce large effects. Dalton and Ingen-Housz offer us examples of men who, having once adopted positions on apparently minor scientific points, found inescapable the attitudes they later assumed toward much larger scientific issues. Similarly small details of extra-scientific history are, presumably, no less important in determining the whole "set" of individual attention and endeavor, both in and out of science. As an example, consider the vivid juxtaposition of a Luther and a Copernicus that Dingle presents.

At the junction of the fifteenth and sixteenth centuries two Churchmen from northern Europe went to Italy. The disgust aroused in one 
produced the Reformation: the stimulus received by the other gave the Renaissance its most pregnant symbol. Truly, we see what our eyes are fitted to perceive.

One thing, forever insufficient in itself, is forever necessary as preliminary to creative thinking: hard work. The scientist who would be an innovator of ideas must be sufficiently motivated for the sustained effort of "years of seeking in the dark for a truth that one feels, but cannot express; . . ." (Einstein). He must be motivated, too, for the concentrated effort suggested by Polanyi's anecdote of Pavlov.

Asked by his pupils in jest what they should do to become "a Pavlov," the master answered in all seriousness: "Get up in the morning with your problem before you. Breakfast with it. Go to the laboratory with it. Eat your lunch with it. Keep it before you after dinner. Go to bed with it in your mind. Dream about it."

Few are they with even the intellectual stamina required of potential Einsteins and Pavlovs; far fewer they who actually become Einsteins and Pavlovs. The variables elude us: we do not even know in what degree Pavlovs and Einsteins are born, in what degree made. Perhaps, however, we may ultimately learn to gauge the end effect of many of the human variables, from the development of studies suggested by those from which Barron concludes that:

. . . creative individuals have a positive liking for phenomenal fields which cannot be assimilated to simple principles of geometric order and which require the development, or, better, the creation, of new perceptual schemata which will re-establish in the observer a feeling that the phenomena are intelligible, which is to say ordered, harmonious, and capable of arousing the esthetic sentiment.

- there appears a positive preference for what we are accustomed to call disorder, but which to them [creative individuals] is simply the possibility of a future order whose principle of organization cannot be told now.

\section{THE BIRTH OF INSIGHT}

Manifold are the ways a new idea is born. Consider the two extremes of a continuous spectrum of possibilities. At one extreme we have the innovator who, after long preliminary study, acquires some dim feeling he cannot yet formulate explicitly. Under the impulse of this feeling, certain shadings of emphasis and neglect appear in the men- 
tal field, which may also undergo some explicit local reworking. Particular features become progressively more prominent, others are progressively obscured, until at last a new conceptual pattern wins through to clear expression. The entire development is characterized by its gradualness. At the other extreme we have the innovator who, after apparently profitless preliminary study, experiences a sudden flash of insight that lights up an entirely new conceptual structure. Such a development-occurring most frequently, but not exclusively, when the problem is a limited one-is characterized by its abruptness and apparent spontaneity.

Either of the indicated extremes may be held to represent what "really" happens always. Taking the first extreme as the general case, one supposes that in apparently discontinuous developments we are aware only of one (or more) high point(s) in what is, however, a completely gradual process. Taking the second extreme as the general case, one supposes that the apparently continuous developments are made up of very large numbers of essentially discontinuous small spurts of insight. The evidence for choice between these and other possibilities seems insufficient, and I find no pressing need to choose.

Events falling at or near the second extreme are particularly striking. A classic example of such a "Eureka episode" is given by Poincaré, who had for some time engaged himself in intensive studies of the Fuchsian functions.

Just at this time I left Caen, where I was then living, to go on a geologic excursion under the auspices of the school of mines. The incidents of travel made me forget my mathematical work. Having reached Coutances, we entered an omnibus to go some place or other. At the moment when I put my foot on the step the idea came to me, without anything in my former thoughts seeming to have paved the way for it, that the transformations I had used to define the Fuchsian functions were identical with those of non-Euclidean geometry. I did not verify the idea; I should not have had time, as, upon taking my seat in the omnibus, I went on with a conversation already commenced, but I felt a perfect certainty. On my return to Caen, for conscience' sake, I verified the result at my leisure.

This and other such episodes seem characterized by the sudden emergence of an idea, by which a problem is completely restructured, from a mental state apparently free from conscious concern with the problem solved. 
A parallel illustration from a very different context is reported by Nicolle, who had been long tormented by his inability to solve this mystery: typhus, violently contagious outside his hospital in Tunis, was apparently noncommunicable within its walls.

One day, just like any other, immersed no doubt in the puzzle of the process of contagion in typhus, in any case not thinking of it consciously (of this I am quite sure), I entered the doors of the hospital, when a body at the bottom of the passage arrested my attention.

It was a customary spectacle to see poor natives, suffering from typhus, delirious and febrile as they were, gain the landing and collapse on the last steps. As always I strode over the prostrate body. It was at this very moment that the light struck me. When, a moment later, I entered the hospital, I had solved the problem. I knew beyond all possible doubt that this was it. This prostrate body, and the door in front of which he had fallen, had suddenly shown me the barrier by which typhus had been arrested. For it to have been arrested, and, contagious as it was in entire regions of the country and in Tunis, for it to have remained harmless once the patient had passed the Reception Office, the agent of infection must have been arrested at this point. Now, what passed through this point? The patient had already been stripped of his clothing and of his underwear; he had been shaved and washed. It was therefore something outside himself, something that he carried on himself, in his underwear, or on his skin, which caused the infection. This could be nothing but a flea. Indeed, it was a flea. The fact that I had ignored this point, that all those who had been observing typhus from the beginnings of history (for it belongs to the most ancient ages of humanity) had failed to notice the incontrovertible and immediately fruitful solution of the method of transmission, had suddenly been revealed to me.

Such a report is the fruit of introspection, notorious for its unreliability. Yet these episodes are reported quite independently, and from quite varied contexts, in strikingly similar terms. And, though not numerous, such episodes are frequent enough to suggest some degree of generality. In the modern era they are reported by mathematical physicists like Newton (the apple), Gauss, Helmholtz, W. R. Hamilton (who had inspiration while crossing, literally as well as figuratively, what thereafter he called Quaternion Bridge), and Poincaré; by the chemist Kekulé (two separate reveries or dreams); by the geologist Kropotkin; and by biological workers like Darwin 
and Wallace (reading Malthus), Metchnikoff, and Nicolle. Poincaré views the sudden inspiration as only a terminal event in a process that continues, throughout an apparently effortfree interim period, once it has been initiated by intense (though fruitless) conscious efforts to solve a problem.

These efforts then have not been as sterile as one thinks; they have set in motion the machine of the unconscious, and without them it would not have moved and would have produced nothing.

One would not wish to make overmuch of "the Unconscious"-a disembodied entity within the disembodied entity of the mind. But may we not speculate about "subliminal workings of the brain" (lower case and referring to a genuine noun)? Braithwaite argues for a related conception in terms which seem to me persuasive.

... to give an explanation in terms of processes in an "Unconscious" does not preclude the possibility of also giving an explanation in terms of physico-chemical processes in the brain (or, more generally, in the body). What they [Freud and his disciples] claim is that, although they believe, with the physiologists, that such a physico-chemical explanation may well be discovered in the future, at the present time, with our ignorance of the detailed workings of the brain, an explanation in terms of an Unconscious is more profitable in enabling predictions to be made. . . . On the other hand, many neurologists have rejected out of hand any explanation in terms of unconscious states of mind on the ground that these are unobservable and are therefore mythical entities, without appearing to realize that, if such entities occur as theoretical concepts in the highest-level hypotheses of a scientific deductive system conclusions from which are empirically confirmed, they have exactly the same epistemological status as the electrical and chemical concepts in terms of which the neurologists would wish to give their physico-chemical explanation.

One may then perhaps hypothesize subliminal thought at least partly freed from some of the constraints imposed by our waking conception of "the possible." Categories and classifications ordinarily definitive are somewhat dissolved, logical connections ordinarily indubitable are loosened, certain habitual implicit assumptions perhaps drop entirely from view. The rigidity of a pattern of thought is relaxed, its contents are freed to mix with other facts and ideas. Now arise possibilities that consciousness would ordinarily "forbid." The 
abrupt crystallization of some such possibility, like the almost explosive freezing of a supercooled lake, breaks into consciousness with the typical Gestalt sensations of sudden release, perfect completeness, and absolute certainty. The sense of certainty may of course prove unjustified. Initiated by hard work of preparation, intuition must be followed by hard work of confirmation-as noted by Kekulé in concluding the account of his own "Eureka experience."

Let us learn to dream, gentlemen, then perhaps we shall find the truth. . . . But let us beware of publishing our dreams till they have been tested by the waking understanding.

Lorenz considers the insight achieved in a "Eureka experience" distinctly superior to anything that conscious reasoning can produce, because

. . . intuition, like other highly differentiated types of Gestalt perception, is able to draw into simultaneous consideration a far greater number of premises than any of our conscious conclusions.

Lorenz may well be right, but I have seen no demonstration that subliminal thought is capable of any feats inaccessible to fully focused thinking. If I look at one of Josef Albers' figures on the frontispiece of this book I can see but one pattern. Blinking, or letting my eyes slide in and out of focus, I suddenly see a wholly different pattern. But I can achieve the same end through the deliberate effort to reconstruct, or "see differently," certain sections of the figure which seem to me awkwardnesses in the first pattern. By the sustained effort of scientific genius apparently definitive categories can be broken up, apparently inseparable logical ties can be ruptured, hidden assumptions can be dredged up to view, a new pattern can be systematically created from pieces of the old. But, equally, subconscious thought may succeed where fully focused thought has not yet prevailed. In either case the ultimate achievement is often the same-and such as to make utterly unrecapturable the original innovator's experience of insight. For as Koestler points out-in his analysis of the work of Archimedes that furnishes the (possibly apocryphal) prototype of all "Eureka" experiences-that insight links forever after what had ever before been separate.

The immense difficulty, the creative originality of this matchmaking is not apparent in the smooth syllogistic schema. The schema gives 
the impression that the mental achievement consisted in drawing the conclusion. In fact, the achievement was to bring the two premises under one roof, as it were. The conclusion is merely the offspring of the marriage, arrived at by routine actions. . . .

... [Thus] all revolutionary innovations appear after a whiie as trivial and obvious, and we marvel less at the discovery itself, than at the apparently abysmal stupidity of the mental stage preceding it: . . . 


\section{CHAPTER XII}

\section{The Real World}

... that which I meditate and propound is not Acatalepsia, but Eucatalepsia; not denial of the capacity to understand, but provision for the understanding truly. - BACON

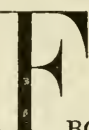

ROM science I turn to cosmology, to argue the case for belief that science discovers to us something of the nature of the real world. I find that belief beneficent, and justified by no inconsiderable body of evidence. By positivists, empiricists, instrumentalists, operationalists, phenomenalists, and others of the Pyrrhonist tribe, the evidence is ignored, the belief dismissed as "meaningless," and reality cast aside as "only a comfort word." Bohr pronounced as well as any the central credo of this philosophic extravagance.

. . . in our description of nature the purpose is not to disclose the real essence of the phenomena but only to track down, so far as it is possible, relations between the manifold aspects of our experience.

But science has not grown through rejection of the purpose "to disclose the real essence of the phenomena"-which purpose lies very much at the focus of the work for which Bohr will be longest remembered. The reach of science has ever exceeded its grasp, but only as men have been bold enough to reach has their grasp been lengthened. What has in the past, and will in the future, most stir men to scientific achievement is not, I think, the cool disbelief of Bohr but rather that intense conviction voiced by Polanyi.

. . . personal knowledge in science is not made but discovered, and as such it claims to establish contact with reality beyond the clues on which it relies. It commits us, passionately and far beyond our com- 
prehension, to a vision of reality. . . . the kind of order which the discoverer claims to see in nature goes far beyond his understanding; so that his triumph lies precisely in his foreknowledge of a host of yet hidden implications which his discovery will reveal in later days to other eyes.

The pretensions of metaphysics are not curbed but encouraged when scientists abandon their claim "to establish contact with reality." The militant Victorian champions of science argued that it gives us Truth, while metaphysics supplies only confusion. The positivists concurred in the disparagement of metaphysics, and sought to consolidate the attack by showing that even science can make no claim to know Truth. But the net effect of this analysis is to blur, not to sharpen, the distinction. Science and metaphysics are now differentiable only in that statements of science are useful, while those of metaphysics are "meaningless." That distinction is all too readily ignored. Indeed, if neither science nor metaphysics can give us Truth, why not prefer metaphysics to science? If "meaningless," metaphysics has at least "value" whereas science has only vulgar utility. To be sure, positivism does make strange bedfellows. How ironic that beneath its quilt we find, together with the vigorous naturalism of a Mach, the theologic apologism of a Duhem-who finds in positivism the instrumentality that makes metaphysics master of science.

It seems to me that contemporary philosophy of science has not much improved matters. Consider, for example, the following statement by Quine:

As an empiricist I continue to think of the conceptual scheme of science as a tool, ultimately, for predicting future experience in the light of past experience. Physical objects are conceptually imported into the situation as convenient intermediaries-not by definition in terms of experience, but simply as irreducible posits comparable, epistemologically, to the gods of Homer. For-my part I do, qua lay physicist, believe in physical objects and not in Homer's gods; and I consider it a scientific error to believe otherwise. But in point of epistemological footing the physical objects and the gods differ only in degree and not in kind. Both sorts of entities enter our conception only as cultural posits.

If as a logician Quine can argue that Homer's gods and physical objects are fundamentally alike "in point of epistemological footing," I as a chemist might with equal justice maintain that a $\$ 1$ and a $\$ 1000$ 
bill are identical "in point of chemical footing" (i.e., same paper, same ink). Yet in each case I have an uneasy feeling that one is dwelling overlong on a similarity which-though no doubt abundantly interesting in itself-is yet trifling in comparison with the enormous functional difference that is left out of all account. Precisely that neglect is responsible for what Quine himself recognizes to be the thrust of his argument: “. . . a blurring of the supposed boundary between speculative metaphysics and natural science." In my opinion any such "blurring" represents an inordinately high price to pay for epistemological enlightenment. For certainly, if Homeric gods and physical objects "differ only in degree and not in kind," must not religion and science then differ only in degree and not in kind? And if fossil sea shells are then found on high ground, why not believe the Devil put them there to mislead men?

\section{NAÏVE REALISM}

Naïve realism is Samuel Johnson's view that the stone that stubbed his toe thereby established a claim to be considered "real." It is the view of the man of common sense. We saw earlier that, necessarily, it is also in part the view of the microphysicist. Naive realism conceives an external world wholly independent of our knowing of it, progressively revealed to us by science, and certainly and objectively depicted by scientific knowledge. For reasons indicated earlier, and now only briefly recapitulated, this appealingly straightforward position is utterly untenable.

Provisionally adopting the realist position, we must accept the results of a century of physiological and psychological research that demonstrates the great complexity of the "simple" process of perception. In Chapter I we found that "naked perception," free from all conceptual component, is a fiction: the most primitive reports of the "real world" furnished by our senses are already colored by our humanity. A physical object is not a given reality, as the naive realist supposes, but irreducibly involves human inference. Nor is a scientific law, as he supposes, the pure expression of a natural order discovered as such. In Chapters II and $V$ we saw the major elements of conceptual invention that enter into the making of laws quite clearly, then, not perfect general truths dictated by nature to an attentive but passive human auditor.

The naïve realist supposes scientific theories shaped only by the 
data of experience, and selected by purely objective criteria. We saw in Chapter IX that this is a hopeless position. When not enforced simply by cosmologic preferences, our selection is determined by the purely practical effectiveness of theories as heuristic tools in the hands of human investigators. But now consider that, presented with an appearance of qualitative change, our first impulse is always to conceive it in terms of the local displacements of the otherwise unchanging. The heuristic effectiveness of corpuscular theories may then be held to derive at least as much from the structure of the human sense of rationality as from the structure of the "objective" world those theories purportedly depict.

The regulative and substantive principles the naïve realist considers discoveries about the world can instead be regarded as humanly chosen conventions-as Frank regarded the principle of determinism. Accepting that principle, we take it for granted that if a given set of circumstances (state A) is once followed by another set of circumstances (state B), then state A will always be followed by state B. Would we, asks Frank, ever bring ourselves to recognize a failure of the ultimate principle itself, as distinct from our own failures in applying it? Consider, he says, some particular mechanical system in which we determine all the masses, distances, etc., we conceive relevant to determination of state $\mathrm{A}$, and find it succeeded by state $\mathrm{B}$. Consider that in many trials we find state A reproducibly succeeded by state B. Suppose then we encounter some case in which state A is not succeeded by the expected state B! Failing to restore determinism by enlarging the system, to allow for "external influences," do we then reject the principle of determinism? Certainly not! We rush in with postulates of hidden factors, or "state variables," not previously comprised in our description of state A. We hypothesize, for example, electric or magnetic parameters that had one value in earlier trials but some different value when state B was not obtained. And we go on hypothesizing such complications until we have so defined a state $\mathrm{A}^{\prime}$ that, in all our experiments, state $\mathrm{A}^{\prime}$ is followed by state $B$. Here we rest content until, as may happen, we meet an experiment in which the new state $\mathrm{A}^{\prime}$ is not followed by state $\mathrm{B}$. And then once again we set to work to redefine state $A^{\prime}$, in the form of state $\mathrm{A}^{\prime \prime}$, and so on. The principle can never fail, Frank concludes, because we will never let it fail. No discovered truth about the real world, the principle is nothing but a humanly enforced convention. 
Consider, finally-is not the whole quest for intelligibility obviously absurd? We saw in Chapters III and VIII that scientific explanation can never be complete. The postulates of our ultimate theory are not themselves explained. Einstein leaves as unexplained the constancy of the velocity of light, as Darwin leaves the origin of the variations sifted by natural selection, as Newton leaves the origin and mode of action of a universal gravitational force. "Natural science," says Mach,

.. . has to resolve the more complicated facts into as few and as simple ones as possible. This we call explaining. These simplest facts, to which we reduce the more complicated ones, are always unintelligible in themselves, that is to say, they are not further resolvable. . . . People usually deceive themselves in thinking that they have reduced the unintelligible to the intelligible. Understanding consists in analysis alone; and people usually reduce uncommon unintelligibilities to common ones. . . .

What facts one will allow to rank as fundamental facts, at which one rests, depends on custom and history.

Given custom Kant found self-evident the universal force Leibniz had found absurd and which we, with a further history, again come to query.

Common acceptance does not render the "fundamental facts" any less unintelligible. Thermodynamics is a theory exemplary for the small number of its ultimate unintelligibilities, and Bridgman writes:

The first law of thermodynamics properly understood is not at all a statement that energy is conserved, for the energy concept without conservation is meaningless. The essence of the first law is contained in the statement that the energy concept exists . . .

But as such the concept "exists" only in our minds-and there in no very clear form since, as Bridgman adds, "no general meaning can be given to the energy concept, but only specific meanings in special cases." Moreover, the rise of quantum mechanics poses a still sterner challenge to the whole idea of intelligibility. Can we pretend to comprehend "particles" that "jump" from state to state without intermediary "existence," "particles" the number of which "actually present" depends on how closely the system is observed, "particles" that are at other times "waves," and so on? Given such as these, the naïve 
realist's hope to find intrinsically intelligible an objective real world cannot but seem pathetically futile.

\section{PHENOMENALISM}

To believe everything and to doubt everything are, says Poincaré, two facile substitutes for thought. From the extreme of naïve realism we fly to the opposite extreme of phenomenalism, e.g., the opinion that scientific theories are nothing but systems for the economic correlation of observables. And, simply because it defends so little, the phenomenalist position is less susceptible to attack. If it is alleged that modern physics is incomprehensible, the phenomenalist replies. that he does not pretend to find it intelligible. If it is alleged that science is shot through and through with humanly enforced conventions, the phenomenalist answers that of course we can construct our own framework of comprehensive correlation with whatever conventions we choose. If it is alleged that we lack definitive criteria for choice between scientific theories, the phenomenalist asks what criterion can be more fundamental than our own convenience. Our laws are but economic descriptions, and physical objects are "conceptually imported into the situation as convenient intermediaries." What could be simpler?

Suppose this were a logically impregnable position. Even then, as Born notes, we would not be bound to accept it.

Logical coherence is a purely negative criterion; no system can be accepted without it, but no system is acceptable just because it is logically tenable.

Solipsism occupies a position far more easily defended than that of any view granting the genuine existence of other people. Yet few among us are convinced solipsists: we go on wrestling hopefully with the difficult epistemological problems a solipsist would dismiss as "meaningless." To this effort we may be further emboldened by recognition that the phenomenalist position is not free from its own characteristic weaknesses. Consider, for example, the confidence with which Mach writes:

Sensations are not signs of things; but, on the contrary, a thing is a thought-symbol for a compound sensation of relative fixedness. 
But now, if a thing is nothing but a "thought-symbol," how does it happen that there is a "compound sensation of relative fixedness"? One cannot very well dismiss it, as an incidental product of human sensory equipment or human usage of certain "cultural posits," without at once invalidating the tacit assumption that "sensations" are given. But in any case, recalling the evidence noted in Chapter I, what about those sensations allegedly given as the physical object is not? If human inference is involved in conception of the thing, is it not at least as deeply involved in conception of the hypothetically pure "sensation"-then also a mere "thought-symbol"?

Illogical cynicism. The atheist can no more demonstrate the nonexistence of gods than the believer can demonstrate their existence. Though probably no weaker, the logical position of the former is surely no stronger than that of the latter. The naïve realist insists that physical objects "really exist." Mach asserts that all such physical constructs are "convenient fictions." Convenient they surely are but, just as the naive realist is impotent to demonstrate their existence, Mach is impotent to show them fictional by arguing that:

. . . in the investigation of nature, we have to deal only with knowledge of the connexion of appearances with one another. What we represent to ourselves behind the appearances exists only in our understanding, ... .

Because "what we represent to ourselves behind the appearances" efficiently discharges a correlative function, therefore the hypothetical elements exist only in our understanding? Nietzsche comments:

Parmenides said: "One can form no concept of the nonexistent";we are at the other extreme, and say, "That of which a concept can be formed, is certainly fictional."

Positivist arguments bristle with this kind of non sequitur. Our laws and theories contain a substantial human element, and can give us no perfectly objective representation of the world. Therefore they tell us nothing of the world. Quantum mechanics presents a major challenge to human understanding. Therefore human understanding is impossible. Schrödinger speaks bitterly of the "Neo-Machians in present-day quantum mechanics."

Some people believe they have in Mach's principle found a wonderful way out of this dilemma [i.e., the wave-particle dualism] which 
frees us from the obligation to search for clear conceptions of $\mathrm{Na}$ ture by condemning the belief in them as gross superstition.

That "superstition," fortunately, is likely to survive all such attacks on it. At least this is the conclusion Koyré draws from studies of scientific history extending as far back as the Greeks.

... (i) The positivistic phase of renouncement, or resignation, is only a kind of retreat position, and it is always a temporary one; (ii) although the human mind, in its pursuit of knowledge, repeatedly assumes this attitude, it does not accept it as final-at least it has never done so until now; and (iii) sooner or later it ceases to make virtue of necessity and congratulate itself on its defeat. Sooner or later it comes back to the allegedly unprofitable, impossible, or meaningless task and tries to find a causal and real explanation for the accepted and established laws.

\section{REPRESENTATIONALISM}

Between optimistic naïveté and defeatist cynicism there is room and to spare for sanguine scepticism. Never beyond all doubt, scientific knowledge is admittedly never something distinctly separable from the characteristics of the human knower. But, I should think quite as obviously, scientific knowledge also represents (though how I do not pretend to grasp) the characteristics of a real world independent of our knowing of it. I maintain further that as science advances the "purely human" element in our knowledge diminishes, though presumably never to extinction; and that the measure of what remains unexplained also diminishes progressively, though never to zero. For at least part of this contention one finds support from an unexpected quarter. Though he entirely denies the possibility of scientific explanation, Duhem confesses that he (and, he says, every other physicist) finds inescapable the faith that

. . . physical theory through its successive advances tends to arrange experimental laws in an order more and more analogous to the transcendent order according to which the realities are classified, that as a result physical theory advances gradually toward its limiting form, namely, that of a natural classification, ...

Claiming much more than Duhem, I assert an asymptotic approach of scientific knowledge to a faithful representation of the real world. If this view be granted then-turning to my own use an argument 
given by Churchman-I hold that I can legitimately speak of the objective structure of the real world.

In one sense, the "progress" of modern philosophy has been to show that the [cosmologic] objectives of science are not attainable. But there is a viewpoint, typically modern, that to say an objective is unattainable is not the same thing as saying the objective does not exist. This viewpoint would insist that an objective that may be approached within any given distance, however small, exists, even though it can never be reached.

... those questions that are answerable [in an absolute sense] should be answered after an infinity of steps in the process of learning. To make such a concept clear, we might say that the absolute answers are the limits of an infinite series of experiments or observations.

If my view be granted: why should it be? Why not assume that, always, everything is completely hallucinatory? St. Thomas rejected that assumption out of faith that it was not to deceive man that God gave him his senses. I reject the assumption for no stronger reason than that-if not, following Moore, completely incredible-it is at the very least incredibly uninteresting. I cannot then compel assent to my assumption that sensory experience is not purely hallucinatory, nor to the pair of more special postulates that might justify acceptance of the view above expressed.

Our sensory experience is evoked by a cosmos the complexity of which is not incommensurate with the potential power of human comprehension.

The structure of scientific theories is determined by sensory experience and by a human sense of rationality indefinitely educable under the tutelage of experience.

Simply more explicit statements of what is already implicit in the principle of intelligibility, these postulates seem amply justified by two species of considerations.

First: The postulates are meaningful because falsifiable-for example, through a development of the sort Noyes considers probable.

... I have a strong feeling that from now on the mathematical models of theoretical physics are unlikely to be unique, and the adop- 
tion of one rather than another will come to be based more on aesthetic considerations than on experimental criteria. Once this situation actually exists (rather than potentially as is true at present) it would appear to me obvious that the success of physics would have nothing to do with the existence of a hypothetical "real" world.

Aesthetic considerations in scientific judgment are, of course, not a novelty; and they remain entirely legitimate if, as I suppose, they develop from the larger rational sense indefinitely educable. However, I am here concerned not to combat but rather to welcome Noyes' opinion. Precisely because the situation he describes may recognizably materialize, I can until then meaningfully maintain postulates that would be falsified by such an event.

Second: These postulates, like any others worth considering, discharge an explanatory function. Rejecting these postulates, certain data noted hereafter must be either otherwise explained or ignored. But other possible explanations demand other postulates no less numerous and, at least in my opinion, rather less plausible than those I suggest. And the alternate course, of ignoring these data as the phenomenalist customarily does, appears no more satisfactory. The data seem both genuine and germane. Hence, just as we reject a scientific theory that fails to render account of data we conceive a part of "the given" for that theory, I think we must reject a philosophy that fails in similar fashion. But what are the data on which this claim is based? They emerge from a systematic review of each of the points earlier raised against naïve realism.

\section{Beyond Inventions-Discoveries!}

Undeniably involving conceptual invention, a physical object testifies also to an empirical discovery: the existence of "a compound sensation of relative fixedness." A further discovery: these constructs, which according to Mach "exist only in our understanding," generally behave lawfully. But why should lawful behavior ever occur if a scientific law is, as Mach maintains, nothing but an economic description-presumably of "sensations"? The descriptions are no doubt by us invented. But to them corresponds a discovery: economic description is possible; and again the question: why or how is it possible? Weizsäcker stresses that: 
The often cited principle of economy of thought explains, at the most, why we look for simple laws, but not why we find them. . . . We penetrate deeper when we think, at Kant's instance, that order in accord with law is the condition of the possibility of experience; that without the existence of natural laws we could not even form the concepts in terms of which we look for them.

Successful in framing scientific laws, I discover that elements of my experience are orderable. Such a discovery is only to be expected on my postulates-according to which laws will be, in some degree, descriptive of a world sufficiently orderly to be humanly comprehensible. I find in phenomenalist doctrine no explanation whatever of this discovery, the significance of which is not infrequently denied. Thus it may be alleged that my experience is actually completely chaotic but so rich that I can always find in it some elements conformable with some order. James, for example, implies that in framing our constructs we proceed with the same freedom we exercise when we group stars in constellations. But this pretty metaphor is inadequate in the present context. When I form constellations it makes no difference whether I construct a dipper or a bear: I cannot get milk from the one or a pelt from the other, nor do I expect to. The constructs that figure in my laws cannot thus freely be formed, however, for I demand that these laws supply confirmable predictions. A law yielding predictions that are confirmed cannot be reduced to a wholly invented order, like constellations. On the contrary, such a law testifies to an intrinsic orderability of certain elements of experience-precisely that discovery the significance of which I have insisted on.

Science and prediction. Prediction once made, its confirmation depends often on events over which the scientist can exercise no vestige of control. If Thales predicts an eclipse of the sun, Thales' expectation cannot produce the darkness experienced by thousands who did not even know of his expectation. Though I tolerate, and explain away, some failures of prediction, I cannot go on indefinitely "saving" laws that just don't work. The failures of prediction are themselves important; they show our capacity for recognition of such failure. Today we recognize a whole class of (individual microcosmic) events to which the principle of determinism is itself inapplicable. But even here, where our "failure" has been most conspicuous, we can still predict the outcome of statistically large numbers of 
such events with a high degree of reliability which is also precisely predictable.

Accreditation of their claims to foretell the future brought honor to augurs, prophets, soothsayers, oracles, and seers. Shall we not then glory in our creation of the natural science which vastly outstrips them all? Yet, so enormous is the power of scientific prediction, we ordinarily take such power wholly for granted. We should not. However much predictive power may have been sought, its attainability was not assured, and its attainment is a major discovery. Poincaré puts the matter at its very mildest when, after noting that scientific prediction sometimes fails, he adds:

Always the scientist is less often mistaken than a prophet who predicts at random. Besides the progress though slow is continuous, so that, though more and more bold, scientists are less and less misled. This is little, but it is enough.

It is, I think, more than enough to call in question remarks on "the myth of physical objects."

Quine readily concedes the predictive utility of science, and he adds to the statement earlier quoted the further remark that:

The myth of physical objects is epistemologically superior to most [myths] in that it has proved more efficacious than other myths as a device for working a manageable structure into the flux of experience.

To the best of my knowledge, the "myth of physical objects" is not just "superior to most" myths. On the contrary, it is unique among all in leading us to a conceptual structure from which an immense number of confirmable and confirmed predictions are drawn. The laws of science are sought, discovered, and expressed in the terms of this "myth." I know of no substantial body of relations useful for prediction ever sought, found, or expressed in terms of the myth of Homeric gods: indeed, the sibylline utterances of the Delphic oracle make a nice case in point. If epistemology can find between physical objects and gods a difference "only in degree and not in kind," this demonstrates rather less the mythical quality of objects, far more the need for re-examination of the epistemology.

The phenomenal success of scientific prediction points beyond itself, Hertz recognized, to the actual realization of a condition necessary for such success. 
We make ourselves subjective pictures or symbols of external objects. We construct these in such a manner that the logically necessary sequences of these pictures are always symbols for the physical series of represented objects. In order that this be possible, there must be certain concordances between nature and our intellect. Experience teaches us that this process is indeed possible, and so that such concordances do in fact exist.

The concordance so discovered (and uniquely associated with the "myth of physical objects") is neither mentioned nor explained by Quine. Such concordance is of course just what I postulate, just the "congruity" demanded by the principle of intelligibility.

The word "science" derives from the Latin scire, "to know." Science abundantly establishes its claim to know in the sense of Comte's maxim: "savoir, c'est prévoir." Perhaps too it makes some claim to know in the much deeper but intimately related (and surely not then "vulgarly pragmatic") sense of Jesus' "by their works ye shall know them." The picture of nature that supports our search for scientific laws is, says Schrödinger, "not only a permissible tool, but also a goal."

Foretelling, predicting, observation is only a means for us to ascertain whether or not the picture that we have formed is correct.

\section{THEORIES}

Scientific laws are accommodated in scientific theories the postulates of which limn some picture of the world. Are not all such pictures obviously meaningless? Viewing the succession of "revolutionary" advances in scientific theory, we may well seem to pass through a gallery of pictures no two of which are at all alike. And, insofar as they suggested any pictures at all, even such coeval theories as Schrödinger's wave mechanics and Heisenberg's matrix mechanics seemed to represent fundamentally irreconcilable conceptions. Yet ultimately we find that these two theories, each in its own way, depict what we can regard as the "same situation." And, especially when the correspondence principle applies to the historical succession of theories, we find that the highest and progressively more subordinate theories also represent-each in its own way, and with its own degree of generality-the "same situation."

In parrying the first allegation of meaninglessness do we not leave 
ourselves open to a more dangerous renewal of the same thrust? At best the pictures drawn by our theorists are symbolist sketches forever tinged, as Hertz emphasized, by human predisposition to certain modes of symbolization. If then we find an element of sameness in the pictures drawn by successive theories, is this not testimony for the common humanity of the painters, rather than for the faithfulness of their rendition of a common subject? James argues that:

We plunge forward into the field of fresh experience with the beliefs our ancestors and we have made already; these determine what we notice; what we notice determines what we do; what we do again determines what we experience; so from one thing to another, although the stubborn fact remains that there is a sensible flux, what is true of it seems from first to last to be largely a matter of our own creation.

Now if unchanging aesthetic and cosmologic considerations were decisive in our choice of scientific theories, James' position would be very strong. We have seen that in the term of months or years these subjective considerations may indeed be paramount. But irreducibly objective components loom large in the two longer-term criteria of judgment: heuristic power, effective in the period of decades to generations; and simplicity, effective in the period of generations to centuries.

Heuristic power. Entirely denying that scientific theories have distinctive heuristic power, one may argue that the illusion of such power is merely a secondary manifestation of correlative efficiency that puts us in full command of what is already known. But only the heuristic function of the theory makes the old knowledge relevant to discovery of the new, by revealing in the old a pointer toward the new. Take as an example the conception by Maxwell, in mid-19th century, of a kinetic theory of gases involving a particular distribution of molecular velocities. More than half a century later Maxwell's theory leads Stern, and then others, to devise novel systems yielding (velocity) data of a species entirely different from the simple pneumatic data accessible to Maxwell. The new data prove precisely concordant with predictions drawn from Maxwell's theory. This, and other like successes, seem to demand, and thus to testify to, the function of Maxwell's theory as something more than a clever mnemonic device. 
Still denying the significance of a theory's display of heuristic power, one perhaps argues that the appearance of such power reflects no more than the theory's nice concordance with human rationality. Unquestionably significant, this is again a point unquestionably insufficient. As metaphor consider that however nicely the handle of a panel saw may be adapted to my grip, I find it ineffective as a tool for cutting concrete. At its handle end an effectual tool must make some match with its user but, in addition, at its business end it must make some match with that on which it is used. From a theory I easily conceive I may readily draw predictions of new phenomena; but the theory is quite incapable of ensuring the success of my search for them. When, on Newton's theory, I predict a polar flattening of the earth, my expectation does not produce the results that confirm that flattening; when Stern predicts a certain kind of smear of silver on glass, his expectation does not produce the smear. Such heuristic displays are irreducible to manifestations of "subjective" felicity. That a scientific theory sets me to look for many particular things I find seems rather to point to some degree of objectivity in the theory's picture of the world. We can then justifiably generalize the thrust of Cohen's commentary on a particular example: the highly mathematical theory of elastic vibrations.

"What," Mach naïvely asks, "have vibrating strings to do with circular functions?" "But," we may ask in turn, "how could these functions guide us to the discovery of so many physical properties of vibrating strings and other phenomena, if they had nothing to do with the physical facts?" Mach and others are misled by the fact that mathematical functions are not copies of sensational elements. This, however, need not prevent these functions from significantly representing groups of relations which do characterize physical processes.

Still the significance of the alleged heuristic power of scientific theories can be resisted. May it not be argued that all appearance of heuristic power is nothing but an artifact of human memory: we forget all the cases in which predictions of new phenomena failed, with consequent amendment of the theory, and remember as "brilliant heuristic successes" only the remaining cases in which the predictions were borne out? No: that position is, I think, one patently untenable. From the time of Newton to the time of Einstein a veritable galaxy of brilliant discoveries develops from exploitation of the heuristic power 
of Newtonian mechanics-but in this long period I do not see any fundamental revision required, or made, in that system. Suppose even that I have overlooked several such major revisions: are there not obviously many successes of the theory any one of which outweighs a thousand failures? When Adams and Leverrier predict that a new planet will be seen if a telescope is pointed at a certain segment of the sky at a certain time, what are the odds against their success if the Newtonian system is but a clever correlative device? To say the odds are astronomical is ambiguous but correct in every sense. And to dismiss such developments as "chance," when they occur not once but repeatedly, is to masquerade a miracle in the guise of chance.

I maintain that theories selected for their heuristic power are theories selected by an at least partially objective criterion. And the demonstrable heuristic power of major scientific theories I hold to be a genuine discovery demanding explanation. Heuristic power is a fact; a fact unexplained and inexplicable within the phenomenalist view of scientific theory-as nothing but a humanly invented system for comprehensive correlation of economic descriptions of human "sensations" experienced in the past. The obvious bankruptcy of this position is, indeed, just what drove Duhem to his trans-phenomenalist conception of scientific theory as a "natural classification." For Duhem did not fail to recognize that the immense heuristic power of a major scientific theory must signify that it offers some "reflection of the real relations among the invisible realities."

The highest test, therefore, of our holding a classification as a natural one is to ask it to indicate in advance things which the future alone will reveal. And when the experiment is made and confirms the predictions obtained from our theory, we feel strengthened in our conviction that the relations established by our reason among abstract notions truly correspond to relations among things.

Simplicity. On ever-narrower postulational foundations, we endeavor to construct scientific theories of ever-increasing scope and precision of correlation. Human desire explains why we seek such theories but, as Weyl observes, not why we are able to find them.

... the farther the analysis progresses, the more detailed the observations become and the finer the elements into which we dissect the phenomena, the simpler-and not the more complicated, as might 
be expected-become the basic laws [i.e., theoretic premises], and the more completely and accurately do they explain the factual course of events.

The attainability of such simplicity is again a discovery, a fact to be explained. And, like heuristic power, this is again a fact explicable, I think, only on the basis of the postulates I have proposed: though humanly invented and appraised, scientific theories do, in increasing degree, represent the structure of a real world not too complex to be humanly comprehensible.

A choice between geocentric and heliocentric theories of planetary astronomy cannot be made reliably a priori. However, with the longterm accumulation of observations, we find that the geocentric system can be "saved" only by ceaseless expansion of its postulational foundation. We find no necessity whatever for this kind of complication of the heliocentric theory. We readily understand that any theory can always be saved by addition of further postulates: such a theory may be handsome testimony to human inventive talent. But a theory which involves little or no such salvage operation seems to testify to something more than human inventiveness, and so to be singled out by something more than human predilection. The phenomenon of calcination offers a parallel example. A priori we have no firm basis for judging whether, in this process, something is added to or subtracted from the metal. But we find that with the long-term accumulation of data phlogiston-type theories become hopelessly complex, while the oxygen theory does not. To dismiss our choice of the oxygen theory as nothing but a matter of human subjectivity is then nothing but nonsense.

Our subjective preference is for simplicity. Our at least partially objective discovery is that simplicity is attainable in some ways and not in others. Weyl says:

It often happens that for some partial domain an explanation $\mathrm{A}$ is simpler than B; but while A becomes increasingly complicated as the circle of experience widens, the same does not apply to $B$, with the result that eventually $B$ emerges as the superior theory. Furthermore the required simplicity is not necessarily the obvious one, but we must let nature train us to recognize the true inner simplicity.

Nature's training is too energetic to be ignored. Thus, after noting how quantum mechanics grows out of a supremely confident en- 
deavor to carry through the program of classical physics, Heisenberg calls attention to

. . . the completely unexpected realization that a consistent pursuit of classical physics forces a transformation in the very basis of this physics, ... .

On the basis of many such occurrences, Einstein concludes that:

The historical development has shown that among the imaginable theoretical constructions there is invariably one that proves to be unquestionably superior to all others. Nobody who really goes into the matter will deny that the world of perceptions determines the theoretical system in a virtually unambiguous manner, although no logical way leads to the principles of the theory.

The burden of proof lies with any who would deny this conclusion. Leaving open to them any and all means short of the inane expedient of dismissing the bulk of our experience as hallucinatory, let them be challenged to produce some reconstruction of, say, modern physical theory which attains as high a correlative index as that already achieved.

We saw in Chapter IX that an entire complex of laws and theories is involved in any comparison of hypothesis and experiment-just as Duhem maintained.

... hypotheses shall be chosen in such a manner that from them taken as a whole mathematical deduction may draw consequences representing with a sufficient degree of approximation the totality of experimental laws. . . . The two systems must be taken in their integrity: the entire system of theoretical representations on the one hand, and the entire system of observed data on the other. As such they are to be compared to each other and their resemblance judged.

Thus a mismatch between theoretic prediction and experimental finding can always be repaired by making a change anyuchere in the theoretic constellation. But, just because a large theoretic system is involved in all such comparisons, each change has potentially some effect everyuchere. In the long term we find that, though there are numberless ways of "saving" any theoretic statement or group of statements, there is one theoretic constellation superior in correlative index to any others we have conceived.

The involvement of a large theoretic constellation in any and all 
comparisons with experiment is actually positively advantageous. For example, it often allows us to resolve situations of deadlock existing within the domain of one particular science. Thus for some years no astronomical observation was competent to resolve the deadlock of Copernican and Tychonic astronomies. But the deadlock in astronomy is broken by the Newtonian reconstruction of physics. Together Copernican astronomy and Newtonian physics form a coherent theoretic system of immense correlative index; and nothing of the sort is found possible when Tychonic astronomy is "held true." Such considerations determine also the acceptability of our most fundamental substantive and regulative principles.

\section{PRINCIPLES}

In the short term, scientific work is dominated by principles we are resolved to maintain come what may. Were they invariably held true then indeed science might tell us far less of the nature of the world than of ourselves, who choose and enforce these principles. Theories shaped to conform with such principles guide our observations and experiments, and the interpretation of the results so obtained is directed by the selfsame principles. Can we then at all hope to recognize a failure of some such principle? Of course we can: demonstrably our principles have changed! Meyerson comments:

It is we who try to establish identity in nature, . . . And that is what we call understanding and explaining nature. It yields itself to a certain extent, but it also resists. Reality rebels and does not allow us to deny it.

Meeting resistance and rebellion, and bringing ourselves to recognize it as such, we do "let nature train us" even as regards articles of scientific faith as fundamental as those noted by Weizsäcker.

Thus we apply successively to our measuring instruments Euclidean geometry, the law of causality, and the category of substances; but it is with these very instruments that we discover phenomena for which those same principles are no longer adequate.

How does such inadequacy manifest itself? In the 18th century Kant felt that Euclidean geometry could never be challenged, because we cannot view the world save in Euclidean terms. Even at the beginning of the 20th century Poincare felt that Euclidean geometry 
would never be challenged-because to the human sense of rationality it must always seem the simplest of geometries, and so be chosen and enforced as a matter of convention. Both were wrongPoincaré because he had failed duly to appreciate Duhem's point. In science we judge the aggregate correlative index of an entire theoretic constellation of which the geometry is but one part. However simple in itself, Euclidean geometry can be maintained, we find, only by introducing serious complications elsewhere in the constellation of theories. Thus at last we feel driven to conceive Euclidean geometry subordinate to Riemann's, and we are so driven by discovery of something sufficiently objective to fall quite beyond the control of a very strong human predilection.

The principles today provisionally conventionalized simply cannot be dismissed as purely human inventions. Always they turn on an irreducible element of substantive discovery. The principle of determinism Frank supposed humanly unchallengeable is today challenged-but not until long after Einstein had pointed out the genuine discovery that still makes the principle more than a convention. Frank reports that Einstein

. . . agreed with me that, whatever may happen in nature, one can never prove that a violation of the law of causality [i.e., the principle of determinism] has taken place. One can always introduce by convention a terminology by which this law is saved. But it could happen that in this way our language and terminology might become highly complicated and cumbersome. What is not conventional . . . is the fact that we can save this law by using a relatively simple terminology: we are sure that a state $\mathrm{A}$ has recurred when a small number of state variables have the same values that they had at the start. This "simplicity of nature" is the observable fact which cannot be reduced to a convention on how to use some words.

The concept "energy" is no doubt a human invention, and nothing originally excluded the possibility that a limitless variety of "species of energy" would be required for the conservation we insist on maintaining. Yet, though we must postulate a considerable number of species, the number is still remarkably small. This is a discovery and it invests the energy principle with a status more substantial than that of any purely human convention. Consider a second example. Doubtless the contemporary human sense of rationality powerfully enforces the regulative principle that qualitative change is to be con- 
strued in terms of the local displacements of the unchanging. But our preference for corpuscular theories has also a strongly "objective" justification. Our pursuit of such theories does not ensure that we will not have indefinitely to multiply species of "particles," the "qualities" thereof, and the "kinds" of local displacements-much as we find it necessary to multiply Ptolemaic epicycles, states of phlogistication, or, for that matter, caprices of Homeric gods. That corpuscular thinking leads to theoretic systems of immense correlative index is not our invention but our discovery-and this not just a discovery about ourselves but also about the world.

The cross-fix. From a shadow cast on the wall of a cave we infer the existence of some specific object ouside. Going outside the cave, we easily observe whether our inference has been correct. Whether atoms, electrons, and other such inferred entities are "real" is, however, an issue not as simply resolved. For in this case we can find no operation cognate with "going outside the cave," to look at the thingin-itself. Even so, Born's deeper exploration of the metaphor strongly suggests that certain other important resources are still open to us.

Cut out a figure, say a circle, of a piece of cardboard and observe its shadow thrown by a distant lamp on a plane wall. The shadow of the circle will appear in general as an ellipse, and by turning your cardboard figure you can give to the length of an axis of the elliptical shadow any value between almost zero and a maximum. That is the exact analogue of the behavior of length in relativity which in different states of motion may have any value between zero and a maximum. . . . the simultaneous observation of the shadows on several different planes [of known mutual inclination] suffices to ascertain the fact that the original cardboard figure is a circle and to determine uniquely its radius. The radius is what mathematicians call an invariant for the transformations produced by parallel projection. . . . Most measurements in physics are not directly concerned with the things that interest us, but with some kind of projection, this word taken in the widest possible sense. . . .

This description applies to any quantum effect. An observation or measurement does not refer to a natural phenomenon as such, but to its aspect from, or its projection on, a system of reference which as a matter of fact is the whole apparatus used. . . .

The main invariants are called charge, mass (or rather: rest-mass), spin, etc.; and in every instance, when we are able to determine these quantities, we decide we have to do with a definite particle. I main- 
tain that we are justified in regarding these particles as real in a sense not essentially different from the usual meaning of the word.

I do not know what Born takes to be "the usual meaning" of real. But the human search for invariants, conducted within the boundary condition imposed by the demand for an over-all system of high correlative index, cannot ensure its own success. The materialization of invariants in a great variety of "projections" of subatomic particles is then a genuine discovery. But now, if ordinary physical objects are mythical, how much less even than legendary will be those subatomic particles. Yet there is nothing mythical about the invariance they display. That is a fact demanding explanation, a fact inexplicable in terms of myths, a fact explained if (with Born) we suppose that the conceptual entities do have some kind of real cognates.

Born's "simultaneous observation of the shadows on several different planes" yields us a cross-fix that assures us that we have indeed found an invariant. Just so, when concordant values of atomic constants emerge from wholly distinct bodies of data, the cross-fix so established is, as Born notes, of great significance.

The kinetic interpretation of the deviations from Boyle's law leads to an estimate of the size of the molecules, which is confirmed by a quite different set of phenomena, the irreversible processes of heat conduction, viscosity, diffusion. Many concepts first introduced in a theoretical way, like velocity distribution, free path, etc., have been confirmed and determined by direct measurements. The fluctuations predicted by the kinetic theory are observable in many ways, through the Brownian motion, the blue color of the sky, etc. . . . the kinetic theory leads to definite properties of the molecules, weight, size, shape (degrees of freedom), mutual interaction. A small number of molecular constants determines an unlimited number of phenomenological properties, in virtue of the molecular hypothesis. Therefore each new property predicted is a confirmation of the molecular hypothesis.

A confirmation perhaps particularly impressive is Einstein's success in predicting on theoretical grounds the yet-to-be-discovered law of a Brownian motion which (though discovered some 75 years earlier by the botanist R. Brown) Einstein did not even know to exist as a phenomenon. To be sure, no aggregate of such confirmations can demonstrate the real existence of particles objectively like those we 
conceive. But such confirmations do, I think, add up to a problem perhaps worthy the attention of those who maintain that such particles "exist only in our understanding."

Some may wish entirely to deny that cross-fixes are significant, because there is some necessary concordance of theories and experiments alike made by human scientists. But recognizing the argument, one recognizes too that it is devoid of weight until how such concordance necessarily arises is revealed by a demonstration yet to be produced and, in my opinion, most unlikely ever to be produced. For consider just those most remarkable cross-fixes that involve the characteristically different data, assumptions, and theories of different sciences. In the 19th century relative atomic weights were derived by chemists from chemical data and a number of special assumptions of simplicity. In the 20th century substantially the same set of (average) relative atomic weights was derived by physicists from data on discharge tubes interpreted with the aid of a quite different body of postulates. What are the odds against such success if chemical and physical theories do not represent, each in its own way, some element of reality? Or again, characteristically chemical data characteristically interpreted by 19 th century chemists led them to impute certain structural configurations to various organic compounds. In the 20th century the physicist, using X-ray diffraction data and a different set of theories and assumptions, is led quite independently to the assignment of almost exactly the same structures. And so on. "Chance," acceptable as explanation of one highly improbable event, is quite insufficient to explain the many such as these.

To argue that humanly invented and selected scientific principles and theories in some measure depict the structure of the real world is, perhaps, to ask acceptance of a miracle. But consider the force of Hume's dictum:

. . . no testimony is sufficient to establish a miracle unless the testimony be of such a kind, that its falsehood would be more miraculous, than the fact which it endeavors to establish; . . .

I offer as testimony the discoveries that scientific theories of evermore extraordinary simplicity have immense heuristic power and lead to truly remarkable cross-fixes. These are facts explicable, I think, only on the basis of the miracle I propose. Denying this testimony-e.g., regarding scientific objects as myth-one must suppose 
heuristic power and cross-fixing arise by "chance." Given the odds against the overwhelming successes actually achieved, I hold that this denial does demand something "more miraculous" than the achievement I claim for science.

\section{INTELLIGIBILITY}

Always ours will be a characteristically human science, but the significance of this banality is too often vastly overinflated. For, of course, the human sense of rationality that looms so large in science is not at all the immutable absolute Kant thought it. Even quite recently Dingle writes of "Reason, being independent of experience, ..." and doughtily maintains that ". . . we are certain of the laws of thought simply because our imagination of alternatives to them is stillborn." As a matter of record, we have in the last century or so invented and used non-Euclidean geometries, noncommutative algebras, and multivalued logics our remoter ancestors would have held to violate "the laws of thought." Today the quantum mechanicians among us conceive and credit what amateurs still find irrationalities, and what a century or so ago might well have been quite literally inconceivable. The history of science thus approves the judgment of Condorcet, who dwelt on the inestimable perfectability of human reason.

If today the human sense of rationality demands atomistic explanations of qualitative change, this too is something learned from experience, for the ancient and medieval sense of rationality imposed no such demand. If today we adopt certain "laws of thought" they are, as Bronowski observes, in some part discoveries about the nature of a world that can so be thought about.

The world makes sense all right; it makes common sense. . . . But common sense is not what we put into the world. It is what we find there.

If then ours is human science, because dependent on the human sense of rationality, it is not for that a science incapable of supplying some significant representation of a cosmos that shapes our rationality-through processes of physical and conceptual evolution Sherrington remarks as still very actively in progress.

Our world we recognize today as a world in making, and ourselves as a part of it likewise in the course of making. . . . the human mind is 
part of a tide of change which, in its instance, has been latterly and, we may think, still is, running like a mill-race. . . . It is being made along with our planet's making. We do not know that it ever will be finished.

Ultimate unintelligibilities? Because (by the margin of their postulates) scientific theories cannot offer us complete explanation, therefore they offer us no explanation. This seems the tenor of Mach's argument, and it is again a complete non sequitur. Quite evidently a few stipulated postulates do explain a vast multitude of derivative laws and concepts. Furthermore these explanations do satisfy a criterion of genuineness not met by explanations in terms, say, of Homeric gods. Consider that the ferocity of the warrior is then ascribed to the inspiration of fierce Ares; the passion of the lover, to amorous Aphrodite; the insight of the sage, to wise Athena; and so on. To gods generally are imputed the very qualities that are to be explained: the problems at issue are, as it were, "pushed upstairs." It is not so in science. Here explanation is both genuine and continuously progressive.

As a great many lesser theories, all with their own postulates, are displaced or subordinated by a few more comprehensive theories, progress in scientific explanation becomes obvious in the reduced number of postulates that must be stipulated but are not themselves explained. Less obvious but not less significant, I think, is a progressive change in the character of the stipulated postulates. More and more these tend to assert identities-ranging from the constancy of the velocity of light and the form of physical laws postulated by relativity theory, to the immutability of atoms hypothesized to explain qualitative change. Meyerson suggests that postulated identities don't need further to be explained. For is not identity an ultimate explanation we find wholly intelligible in itself? Rejecting atomic theories on other grounds, Mach would of course insist that the immutability (i.e., identity in time) of the bodies they postulate is only a common un-intelligibility. But I find that Mach offers, and I suspect he could offer, no demonstration of unintelligibility. And the burden of proof still lies hard upon his followers, since the demonstrated heuristic power of atomic theories strongly suggests that, in fact, we find their postulates quite clearly enough intelligible to draw from them predictions of strange things totally unknown but later discovered. 
The terminal argument against any realist position is this: does not quantum mechanics produce conceptual enormities that explode forever the absurd human pretension to find the world intelligible? The most-cited evidence is, no doubt, the disturbing dualism of wave and particle. Deferring to the opinion of the vast majority of contemporary physicists, let us suppose that, to render a full account of the electron, we must make use of both wave and particle analogies our everyday experience teaches us to regard as fundamentally incompatible. Even as a fact this would not entail the conclusion that the concept "electron" is riven by some deep antinomic flaw. Observe that the incompatability is at most between two analogies we introduce to help us think about a microcosmic entity unlike anything encountered in everyday experience. Actually the apparently contradictory analogies do help physicists to so integral a concept of the electron that many can, with Born, think of it as an entity essentially real "in the usual meaning of the word."

How much of the mystification connected with quantum mechanics arises from our insistence on using words where, as Bohr observes, words simply won't suffice!

. . . all our ordinary verbal expressions bear the stamp of our customary forms of perception, from the point of view of which the existence of the quantum of action is an irrationality.

The necessarily verbal nontechnical exposition of quantum mechanics may then well make it appear utterly unintelligible. But it is not necessarily unintelligible. Indeed, most of the apparent unintelligibilities never even arise in the necessarily mathematical technical exposition that is the proper vehicle of the theory. Those with some bitter youthful experience of mathematics may distrust the implication that there are things and situations that words make obscure and mathematics clear. Yet, far from the dread realm of quantum mechanics, Cohen indicates that, with our own hands, we can easily produce just such things and situations.

. . how many people can imagine a piece of paper that does not have two distinct sides; or a vessel where there can be no line of demarcation between inside and outside? Moreover, when we actually produce the former of these (a Möbius strip or Kummer surface) and ask what will happen if it is repeatedly cut through the middle, the 
imagination unaided by mathematical considerations generally breaks down.

In one minute I can make a Möbius strip which, in a few minutes, I can cut repeatedly. Words just will not convey-but mathematics will-the real essence of what is done by these simple operations.

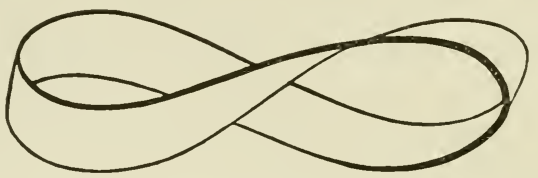

A Möbius strip

The advent of physical theories that demand use of mathematics in place of words indicates neither the unreality of the things and situations only so describable, nor even the ultimate defeat of the quest for understanding. Quite the contrary, it represents a triumphant transcendence of the bounds to human understanding set by everyday language. Consider the statement with which Dirac introduces his quantum mechanics.

Her [nature's] fundamental laws do not govern the world as it appears in our mental picture [of particles, fluids, fields, etc.] in any very direct way, but instead they control a substratum of which we cannot form a mental picture without introducing irrelevancies. The formulation of these laws requires the use of the mathematics of transformations. ... .

. . . The new theories, if one looks apart from their mathematical setting, are built up from physical concepts which cannot be explained in terms of things previously known to the student, which cannot even be explained adequately in words at all. Like the fundamental concepts (e.g., proximity, identity) which every one must learn on his arrival into the world, the newer concepts of physics can be mastered only by long familiarity with their properties and uses.

Note carefully that Dirac does not say that the new concepts are incomprehensible. He likens them to such more familiar concepts as proximity and identity-which we all find conceivable, though they cannot be defined verbally without circularity. The newer concepts can be mastered, though they are difficult and verbally inexpressible; can be mastered, though difficult to imagine without introducing 
irrelevancies. The mathematics is not the means to conceal the incomprehensible. It is merely the agent that permits us to deal with the verbally inexpressible. Not halted at some "natural" limit, we are instead set free to pursue still further that reckless endeavor to grasp the world which is the enterprise of science.

And the pursuit whose quest is Nature's understanding, has this among its rewards, that as it progresses its truth is testable. Truth is a "value." The quest itself therefore is in a measure its own satisfaction. We receive the lesson that our advance to knowledge is of asymptotic type, even as continually approaching so continually without arrival. The satisfaction shall therefore be eternal.

-SHERRINGTON 



\section{Bibliograph!y}

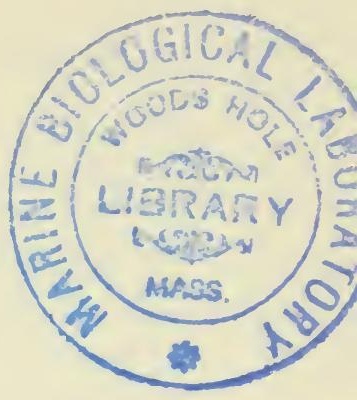

Quoted authors are listed alphabetically. After each name follow specifications of the sources from which quotations have been taken. When more than one work by the same author is cited, each may be identified by a Roman numeral. To find the source of any given quotation, note the number of the page on which it begins and the name of the quoted author. Page numbers of this book are printed here in boldface type. Under the appropriate name, find the pertinent boldface page number and, beside it, an indication of the source of the quotation.

Arago, Dominique François. French physicist (1786-18.53)

256 quoted by Philipp Frank in Philosophy of Science (PrenticeHall, Englewood Cliffs, N.J., 19.57), p. 19.5

Archimedes. Greek mathematician (fl. 250 в.с.)

70 quoted by S. Sambursky, op. cit., p. 221

Aristotle. Greek philosopher (fl. 350 B.c.)

77 Physics I-1-184a

Arrhenits, Svante. Swedish chemist (1859-1927)

316 quoted by L. H. Cragg \& R. P. Graham in Principles of Chemistry (Rinehart, New York, 19.54), p. 411

Ayer, Alfred Jules. British philosopher (1910- )

The Problem of Knowledge (Macmillan, London; St. Martin's, New York, 19.56) 212/126

BACON, Francis. English statesman and philosopher (1561-1626)

Philosophical Works, ed. J. M. Robertson, Ellis \& Spedding, (Dutton, 
New York, 1905) 76/261; 103/quoted by C. Sherrington, op. cit., p. 15; 196/57 [I have preferred the translation cited by A. J. Hopkins in Alchemy: Child of Greek Philosophy (Columbia Univ. Press, New York, 1934), p. 213]; 356/299

BARron, Frank. American psychologist (1922- )

Article in Scientific Creativity, ed. C. W. Taylor \& F. Barron (Wiley, New York, 1963) 350/155-156

BARTLETT, Frederic C. British psychologist (1886- )

Thinking (Allen \& Unwin, London, 1958) 13/86, 156/127, $281 / 122,319 / 11$

Baudelaire, Pierre Charles. French poet (1821-1867)

344 /quoted by René Dubos in The Dreams of Reason (Columbia Univ. Press, New York, 1961), p. 122

Bernard, Claude. French physiologist (1813-1878)

Introduction to the Study of Experimental Medicine, tr. H. C. Greene (Constable, London; Dover, New York, 1957) 39/54, 142/150, $159 / 34-35,176 / 201,205 / 35-36,223 / 48$ (variant translation), $323 / 168,325 / 169,329 / 38,340 / 170,342 / 69-70 \& 166,342 / 33$

Bernoulli, Johann. Swiss mathematician (1667-1748)

186/quoted by E. Meyerson, op. cit., p. 191

Berzelius, Jöns Jakob. Swedish chemist (1779-1848)

332/quoted by Ida Freund in The Study of Chemical Composition (Cambridge University Press, 1904), p. 597

Beveridge, W. I. B. Australian pathologist (1908- )

The Art of Scientific Investigation (Norton, New York, 1950?)

$311 / 48,331 / 48-49,340 / 46,344 / 64$

Binkley, Robert C. American social scientist (1897-1940)

Realism and Nationalism: 1852-1871 (Harper, New York, 1935)

106/19

BLACK, Joseph. Scottish chemist (1728-1799)

Lectures on the Elements of Chemistry, vol. 1 (Edinburgh, 1803) $270 / 192,273 / 489$

Bohм, David J. American physicist (1917- )

Causality and Chance in Modern Physics (Routledge \& Kegan Paul, London, 1957) 202/96 
BoHr, Niels. Danish physicist (1885-1962)

Atomic Theory and the Description of Nature (Cambridge Univ. Press, 1934) 30/96, 285/97, 356/18, 381/19

Bois-Reymond, Emil H. du. German physiologist (1818-1896)

Über die Grenzen des Naturerkennens (Leipzig, 1898) 187/16

Born, Max. Anglo-German physicist (1882- )

(I) Experiment and Theory in Physics (Cambridge Univ. Press, 1943)

(II) Natural Philosophy of Cause and Chance (Oxford Univ. Press, 1949)

(III) Article in Philosophical Quarterly 3 (1953)

(IV) Physics in My Generation (Pergamon, London \& New York, 1956)

29/IV 19-20, 35/II 103-104, 93/IV 124, 101/I 10, 152/I 9, 168/I 44, 245/I 18, 246/I 21, 253/III 140, 361/III 139-140, 376/III 143-146, $377 /$ III 142

Boyle, Robert. British natural philosopher (1627-1691)

187/quoted by Mary Hesse in Forces and Fields (Nelson, London, $1961)$, p. 115; 197 /quoted in the Harvard Case Histories in Experimental Science, ed. J. B. Conant (Harvard Univ. Press, 1957), vol. 1, pp. 57-58

Brain, Walter Russell. British neurologist (1895- )

The Nature of Experience (Oxford Univ. Press, 1959) 172/31-32

Braithwaite, Richard B. British philosopher (1900- )

Scientific Explanation (Cambridge Univ. Press, 1953) 45/354,

235/76, 353/343-344

Bridgman, Percy W. American physicist (1882-1961)

(I) The Logic of Modern Physics (Macmillan, New York, 1928)

(II) The Nature of Physical Theory (Princeton Univ. Press, 1936)

92/II 109, 127/I 42-43, 178/I 53, 230/II 61-62, 248/II 62-63, $360 / \mathrm{I} 127$

Bronowski, Jacob. Anglo-Polish mathematician (1908- )

The Common Sense of Science (Heinemann, London, 1951; Harvard Univ. Press, 1953) 48/48, 83/148, 172/114, 379/78

Brown, George Spencer. Contemporary British mathematician Probability and Scientific Inference (Longmans, Green, London, 1957) $85 / 107$ 
Bruner, Jerome S. American psychologist (1915- )

Bruner, Goodnow \& Austin, A Study of Thinking (Wiley, New York, 1956) 228/104, 229/104, 340/237

Butterfield, Herbert. British historian (1900- )

The Origins of Modern Science (Bell, London, 1949; Macmillan,

New York, 1952) 80/5, 105/viii

Introduction to Arthur Koestler, op. cit. II $\quad 346 / 11$

Campbell, Norman R. British physicist (1880-1949)

What Is Science? (Dover, New York, 1952) 241/96

Churchman, Charles West. American philosopher of science (1913- ) Theory of Experimental Inference (Macmillan, New York, 1948) $364 / 57 \& 169$

Conen, Morris Raphael. American philosopher (1880-1947)

Reason and Nature (Free Press, New York, 1953) 26/278, 163/17, 168/92, 204/85, 205/155, 223/122-123, 344/366, 370/41, 381/74

Coleridge, Samuel Taylor. British poet (1772-1834)

Collected Letters, ed. Leslie Griggs (Oxford Univ. Press, 1956)

65/192-193

Collingwood, Robin George. British philosopher (1889-1943)

The Idea of Nature (Oxford Univ. Press, 1949) 77/95

Comte, Auguste. French philosopher (1798-1857)

$345 /$ quoted by du Noüy, op. cit., p. 80

Conant, James Bryant. American chemist and educator (1893- )

Science and Common Sense (Yale Univ. Press, New Haven, Conn., 1951), $157 / 44,259 / 74,306 / 9$

Свомвie, Alastair Cameron. British historian of science (1915- )

Medieval and Early Modern Science, vol. 2 (Harvard Univ. Press, 1959) $97 / 287-288$

DARwin, Charles. British naturalist (1809-1882)

307 /quoted by René Dubos in The Dreams of Reason (Columbia Univ. Press, New York, 1961), p. 137; 324/letters quoted by Alvar Ellegård in Roots of Scientific Thought, ed. P. Wiener \& A. Noland (Basic Books, New York, 1957), p. 551

De Broglie, Louis Victor. French physicist (1892- )

The Revolution in Physics, tr. R. W. Niemeyer (Noonday Press, New York, 1956) 100/284, 128/20, 202/237-238, 348/quoted by R. Taton, op. cit., pp. 35-36 
Democritus. Greek philosopher (fl. 460 в.c.)

184/quoted by S. Sambursky, op. cit., p. 107

De Santillana, Giorgio. Italian-American historian and philosopher (1902- )

Article in Daedalus 87 (Winter, 1958) 251/48

Dingle, Herbert. British historian and philosopher of science (1890- )

(I) Science and Human Experience (Williams \& Norgate, London, 1931)

(II) Through Science to Philosophy (Oxford Univ. Press, 1937)

(III) The Scientific Adventure (Pitman, London, 1952)

85/I 22, 87/III 40, 174/II 285, 240/I 22-23, 280/III 281,

314/III 211, 315/III 10, 349/III 58, 379/II $178 \& 306$

Dirac, Paul Adrien Maurice. British physicist (1902- )

The Principles of Quantum Mechanics (Oxford Univ. Press, 1930)

$231 / v i, 237 / 18,269 / 7,287 / 1,382 / v$

Doyle, A. Conan. British novelist (1859-1930)

193/A Study in Scarlet, ch. 2

Duhem, Pierre M. M. French physicist and philosopher (1861-1916)

The Aim and Structure of Physical Theory, tr. P. P. Wiener (Princeton Univ. Press, 1954) 96/255, 117/137, 129/172, 241/32-33, 243/19, 347/95, 363/297, 371/28, 373/220

Du Noüy, Pierre Lecomte. Franco-American biologist (1883-1947)

The Road to Reason, tr. Mary du Noüy (Longmans, Green, New York, 1948-courtesy of David McKay Co.) 101/30, 159/77, $190 / 53$

EdDington, Arthur Stanley. British astrophysicist (1882-1944)

The Philosophy of Physical Science (Cambridge Univ. Press, 1949) $96 / 33$

Edelstein, Ludwig. German-American historian of medicine (1902- ) Article in Roots of Scientific Thought, ed. P. Wiener \& A. Noland (Basic Books, New York, 1957) 180/114

Einstein, Albert. German-Swiss physicist (1879-1955)

(I) On the Method of Theoretical Physics (Oxford Univ. Press, 1933): translations slightly amended in light of German text published in Mein Weltbild

(II) Physics and Reality: article in Journal of the Franklin Institute 221 (1936)

(III) Out of My Later Years (Philosophical Library, New York, 1950) 
4/II 349, 25/II 352-353; 28/article in Science 91487 (1940); 47/Einstein \& Infeld, Evolution of Physics (Simon \& Schuster, New York, 1938), p. 33; 103/inscription in Fine Hall, Princeton University; 169/article in Albert Einstcin: Philosopher-Scientist, ed. P. A. Schilpp (Library of Living Philosophers, Evanston, Ill., 1949-courtesy of the editor), p. 684; 173/Ideas and Opinions (Crown, New York, 1954), p. 282; 178/I 12-13, 179/II 351, 218/III 41; 224/Prologue to Max Planck, op. cit. I, p. 10; 285/quoted by G. J. Holton, American Journal of Physics 28634 (1960); 292/III 58, 322/I 5, 328/I 15; 373/quoted by Hermann Weyl, op. cit. II, p. 153

Feigl, Herbert. Austrian-American philosopher (1902- )

Article in Revue Internationale de Philosophie 5 (1951) 262/276

Feyerabend, P. K. Contemporary British philosopher of science

Article in Current Issues in the Philosophy of Science, ed. H. Feigl \& G. Maxwell (Holt, New York, 1961) 295/279

Fierz, Markus Eduard. Swiss physicist (1912- )

Article in Observation and Interpretation, ed. S. Körner (Butterworth, London, 1957) 271/46

Frank, Philipp G. Austrian-American physicist and philosopher (1884- ) Modern Science and Its Philosophy (Harvard Univ. Press, 1950) $375 / 10-11$

Galileo Galilei. Italian physicist and astronomer (1564-1642)

(I) Dialogues Concerning Two New Sciences, tr. Crew \& De Salvio (Macmillan, New York, 1914)

(II) Dialogue on the Great World Systems, tr. Salusbury, ed. de Santillana (Univ. of Chicago Press, 1953)

(III) Discoveries and Opinions of Galileo, tr. S. Drake (Doubleday Anchor, New York, 1957)

44/II 341, 180/II 279, 195/III 272, 197/I 166-167, 212/II 7

HaGgard, Howard W. American physiologist (1891-1959)

Devils, Drugs, and Doctors (Harper, New York, 1929) 71/4

HaHn, Otto. German chemical physicist (1879- )

Article with F. Strassman in Die Naturwissenschaften 27 (1939) $329 / 15$

Hall, Alfred Rupert. British historian of science (1920- )

The Scientific Revolution (Longmans, Green, London, 1956)

64/13, 79/168-169, 80/173, 173/177, 211/18 
Невв, Donald Olding. Canadian psychologist (1904- )

(I) The Organization of Behavior (Wiley, New York, 1949)

(II) A Textbook of Psychology (Saunders, Philadelphia, 1958)

6/II 12, 9/I 7, 12/I 91-92, 12/II 163, 26/I 109, 144/I 41-42, 247/I 118-119

Heisenberg, Werner. German physicist (1901- )

174/quoted by N. R. Hanson in Patterns of Discovery (Cambridge Univ. Press, 1958), p. 212; 289/Physics and Philosophy (Harper, New York, 1958), p. 90; 290/Philosophic Problems of Nuclear Science (Faber \& Faber, London, 1952), p. 42; 373/ibid., p. 1ـ

Heraclitus. Greek philosopher (f. 500 B.c.)

36/quoted by E. Schrödinger, op. cit., II, pp. 207-208

Herschel, John F. W. British astronomer (1792-1871)

Preliminary Discourse on the Study of Natural Philosophy (London, 1840) $45 / 84,69 / 348$

Hertz, Heinrich R. German physicist (1857-1894)

368/quoted by M. Cohen and E. Nagel in Logic and Scientific

Method (Harcourt, Brace, New York, 1934), p. 440

Hesse, Mary B. Contemporary British philosopher of science

Article in British Journal of the Philosophy of Science 2 (1951-2) 232/291, 238/291, 244/291

Hillebrand, William F. American chemist (1853-1925)

111/letter quoted by M. W. Travers in The Discovery of the Rare Gases (Arnold, London, 1928), pp. 56-57

Нооке, Robert. British natural philosopher (1635-1703)

342 /quoted by W. S. Jevons in The Principles of Science (Macmillan, London, 1905), p. 671

Hume, David. Scottish historian and philosopher (1711-1776)

An Enquiry Concerning Human Understanding, ed. L. A. SelbyBigge (Oxford Univ. Press, 1894) 378/115-116

Hutten, Ernest H. Contemporary British physicist

(I) Article in British Journal of the Philosophy of Science 4 (1953-4)

(II) The Language of Modern Physics (Allen \& Unwin, London;

Macmillan, New York, 1956)

231/I 299, 232/II 85, 232/I 290, 239/II 164, 243/II 134,

251/II 201, 288/II 165 
JAMEs, William. American psychologist and philosopher (1842-1910)

Pragmatism (Longmans, Green, New York, 1931-courtesy P. R.

Reynolds \& Son) 3/246-247, 4/129, 17/170, 94/249, 206/61-62, 208/223, 288/61, 306/207-208, 369/255

JEFFREYs, Harold. British astrophysicist (1891- )

Scientific Inference, 2nd ed. (Cambridge Univ. Press, 1957)

$18 / 173,181 / 60,227 / 186$

Kexulé, Friedrich August. German chemist (1829-1896)

354/quoted by F. R. Japp in Chemical Society Memorial Lectures for $1893-1900$, p. 100

Kelvis, William Thomson, first Baron. British physicist (1824-1907)

Notes of Lectures on Molecular Dynamics and the Wave Theory of Light, reported by A. S. Hathaway (Baltimore, 1884) 178/270-271

KePler, Johannes. German astronomer (1571-1630)

78/Astronomia Nova, ch. 19; 309/quoted by M. Polanyi, op. cit. II,

p. 7; 312/quoted by A. Koestler, op. cit. II, p. 332

Keynes, John Maynard. British economist (1883-1946)

Essays in Biography (Hart-Davis, London; Horizon Press, New York, 1951) 323/140-141

Koestler, Arthur. Anglo-Hungarian novelist (1905- )

(I) Insight and Outlook (Macmillan, New York, 1949)

(II) The Sleepwalkers (Hutchinson, London; Macmillan, New York, 1959)

\section{7/II 28, 313/II 21, 319/I 390, 354/I 254-255}

Koyré, Alexandre. Franco-American historian and philosopher (1892- ) Article in The Validation of Scientific Theories, ed. P. Frank (Beacon, Boston, 1954-courtesy of Scientific Monthly) 363/203

Kroeber, Alfred Louis. American anthropologist (1876-1960)

Configurations of Culture Growth (Univ. of California Press, 1944) $276 / 840$

LANDÉ, Alfred. German-American physicist (1888- )

From Duality to Unity in Quantum Physics (Cambridge Univ. Press, 1960) 201/23, 203/vii

Langer, Susanne K. American philosopher (1895- )

Philosophy in a New Key (Harvard Univ. Press, 1942) 8/89,

15/89-90, 33/274, 70/272, 169/8 
Lavoisier, Antoine Laurent. French chemist (1743-1794)

Traité Élémentaire de Chimie (Paris, 1789) 146/Preface-translation in E. Meyerson, op. cit., p. 236; 210/vol. 1, ch. 13-translation in E. Meyerson, op. cit., p. 173

Lewis, Gilbert Newton. American chemist (1875-1946)

(I) The Anatomy of Science (Yale Univ. Press, New Haven, Conn., 1926)

(II) G. N. Lewis \& M. Randall, Thermodynamics (McGraw-Hill, New York, 1923)

206/I 6-7, 224/I 6, 254/II vii

Lorenz, Konrad Z. Austrian naturalist and psychologist (1903- )

Article in Aspects of Form, ed. L. L. Whyte (Lund Humphries, London, 1951) 13/175, 354/177

MACH, Ernst. Austrian physicist and philosopher (1838-1916)

(I) Conservation of Energy, tr. P. Jourdain (Open Court, Chicago, 1911)

(II) The Science of Mechanics, tr. T. J. McCormack (Open Court, LaSalle, Ill., 1942)

$360 /$ I $55-56,361 /$ II $580,362 /$ I 49

Margenau, Henry. American physicist and philosopher (1901- )

The Nature of Physical Reality (McGraw-Hill, New York, 1950)

$11 / 172,11 / 60,65 / 28$

Article in Diogenes (Chicago), Spring 1954 200/78

Maxwell, James Clerk. British physicist (1831-1879)

Scientific Papers, vol. 2 (Cambridge Univ. Press, 1890) 110/742

MCGeOcH, John Alexander. American psychologist (1897-1942)

347/quoted by Hebb, op. cit. I, p. 110

Merton, Robert King. American sociologist (1910- )

Article in American Sociological Review 22 (1957) 304/635

Meyerson, Émile. Polish-French philosopher (1859-1933)

Identity and Reality, tr. K. Loewenberg (Allen \& Unwin, London, 1930) $\mathbf{5} / 22, \mathbf{2 5} / 26, \mathbf{4 6} / 386, \mathbf{1 1 6} / 32 \& 31,374 / 286$

MiLL, John Stuart. British philosopher (1806-1873)

System of Logic, 8th ed. (Longmans, Green, London, 1872), vol. 2 $11 / 186,16 / 200,48 / 208$

On Liberty (Ticknor \& Fields, Boston, 1863) 205/43-44 
NAgEL, Ernest. American philosopher (1901- ) Article in Daedalus 88 (Winter 1959) 298/61

Needham, Joseph. British biochemist, philosopher, and historian (1900- ) 102/quoted by R. S. Cohen in The Validation of Scientific Theories, ed. P. Frank (Beacon, Boston, 1954), pp. 227-228

Newton, Isaac. British mathematician and physicist (1642-1727)

(I) Mathematical Principles of Natural Philosophy, ed. F. Cajori (Univ. of California Press, 1934)

(II) Opticks (Bell, London, 1931), Query 31

173/I 398, 174/I xvii-xviii, 175/II 388, 177/I xvii, 181/I 398, 183/I 413, 183/I 398, 187/II 400, 198/I 547, 198/II 401-402, 206/I 398, 207/II 404, 207/I 400, 212/I 398, 219/I 300 (variant translation), 220/I 302 (variant translation); 223/quoted by C. A. Coulson in Science and Christian Belief (Univ. of North Carolina Press, 1955), p. 41; 226/I 192

NicoD, Jean. French mathematician (1893-1924)

Foundations of Geometry and Induction (Routledge \& Kegan Paul, London, 1930) 170/12, 239/21

Nicolle, Charles J. H. French epidemiologist (1866-1936)

307/quoted by R. Taton, op. cit., p. 30; 352/ibid., pp. 76-77

Nietzsche, Friedrich Wilhelm. German philosopher (1844-1900)

The Will to Power, tr. A. M. Ludovici (Foulis, Edinburgh \& London, 1910) 203/vol. 1, p. 370 [I have preferred the translation cited by P. Frank, op. cit., p. 78], 284/vol. 2, p. 201, 362/vol. 2, p. 50

Noyes, H. Pierre. American physicist (1923- )

Article in American Scientist 45 (1957) 246/438, 246/436-437, $364 / 446$

Oppenheiner, J. Robert. American physicist (1904- )

Article in Foundations for World Order (Univ. of Denver Press, 1949) $4 / 51$

Science and the Common Understanding (Simon \& Schuster, New York, 1953) 103/4-5, 289/74, 294/74

Osiander, Andreas. German clergyman (1498-1552)

329/quoted by Edward Rosen in Three Copernican Treatises (Columbia Univ. Press, New York, 1939), p. 22

Painlevé, Paul. French statesman (1863-1933)

212/quoted by E. Meyerson, op. cit., p. 381 
Pascal, Blaise. French mathematician and philosopher (1623-1662) Pensées 323/section 6, item 395

Pasteur, Louis. French chemist and bacteriologist (1822-1895)

307/quoted by René Vallery-Radot in The Life of Pasteur, tr. R. L. Devonshire (Constable, London; Garden City Publishing Co., Garden City, N.Y., 1927), p. 252; 332/ibid., 443-444; 339/ibid., 79

Peirce, Benjamin. American mathematician (1809-1880)

219/quoted by C. S. Peirce in Collected Works, ed. C. Hartshome \&

P. Weiss (Harvard Univ. Press, 1933), vol. 4, 189

Philolaus. Greek philosopher (fl. 450 B.c.)

177 /quoted by S. Sambursky, op. cit., p. 30

Picasso, Pablo. Spanish artist (1881- )

111/quoted by A. Koestler, op. cit. I, p. 413

Pirie, Norman W. British biochemist (1907- )

Article in British Journal of the Philosophy of Science 2 (1951-2) $190 / 270$

Planck, Max K. E. L. German physicist (1858-1947)

(I) Where Is Science Going?, tr. J. Murphy (Allen \& Unwin, London; Norton, New York, 1932)

(II) The Philosophy of Physics, tr. W. Johnston (Norton, New York, 1936)

(III) The Universe in the Light of Modern Physics, tr. W. Johnston (Allen \& Unwin, London, 1937)

(IV) Scientific Autobiography, tr. F. Gaynor (Philosophical Library, New York, 1949)

196/IV 149, 201/III 82/83, 272/II 97, 285/III 17-18, $327 / \mathrm{I} 214-216$

Plato. Greek philosopher (fl. 380 в.c.)

76/Republic $7530 ; 101 /$ Laws 10 886. [Translation is that given by P. Frank in an article in Proc. of the Amer. Acad. of Arts and Sciences 8016 (July 1951)]

PoINCARÉ, Henri. French mathematician and physicist (1854-1912)

The Foundations of Science, tr. G. B. Halsted (Constable, London; Science Press, New York, 1921) 21/129, 44/326-328, 105/367, $109 / 363$ \& 128, 121/128-129, 126/332-334, 129/351 [I have preferred the translation given by P. Frank, op . cit., p. 69], 165/370-371, 186/121, 210/375, 217/73; 224/quoted by Beveridge, op. cit., p. 84; $279 / 365,296 / 219,351 / 387-388,353 / 389,367 / 324$ 
Polanyi, Michael. Anglo-Hungarian chemist, economist, and philosopher (1891- )

(I) Science, Faith, and Society (Oxford Univ. Press, 1946-courtesy of the Court of the University of Durham)

(II) Personal Knowledge (Routledge \& Kegan Paul, London; Univ. of Chicago Press, 1958)

10/II 96-97, 14/II 101, 22/II 81, 37/II 19-20, 38/II 352, 40/II 44, 97/II 322, 157/II 143, 158/II 277, 167/II 144, 227/II 118-119, 251/II 277, 272/II 151, 279/II 138, 280/II 277, 299/I 42-45, 300/I 34, 30I/I 37, 302/I 36, 303/I 54, 320/II 53, 320/I 29-30, 322/II 169, 326/II 276, 338/I 17, 339/I 76-77, 349/II 104-105, $350 /$ II $127,356 /$ II 64

Popper, Karl R. Anglo-Austrian philosopher (1902- )

The Logic of Scientific Discovery, 2nd ed. (Hutchinson, London; Basic Books, New York, 1959) 16/421, 45/111, 61/59, 295/280

Priestley, Joseph. British clergyman and natural philosopher (17331804)

111/quoted in the Harvard Case Histories in Experimental Science, ed. J. B. Conant (Harvard Univ. Press, 1957), vol. 2, p. 361; 331/ibid., vol. 1, pp. 92-93

Quine, Willard V. O. American philosopher (1908- )

From a Logical Point of View (Harvard Univ. Press, 1953)

$261 / 42-43,357 / 44,358 / 20,367 / 44$

Reichenibach, Hans. German-American philosopher (1891-1953)

(I) Article in Albert Einstein: Philosopher-Scientist, ed. P. A. Schilpp (Library of Living Philosophers, Evanston, Ill., 1949-courtesy of the editor)

(II) The Rise of Scientific Philosophy (Univ. of California Press, 1951)

$295 /$ I $292,311 /$ II $118,328 /$ I 292

Rheticus, George Joachim. German mathematician and astronomer (1514-1576)

226/quoted by Edward Rosen in Three Copernican Treatises (Columbia Univ. Press, New York, 1939), p. 163

Richet, Charles Robert. French physiologist (1850-1935)

The Natural History of a Savant, tr. O. Lodge (Dent, London, 1927) $333 / 110$ 
Rogers, Carl R. American clinical psychologist (1902- )

Article in Etc., Review of General Semantics 11 (Summer 1954)

$333 / 254$

Rosenfeld, Léon. Belgian physicist (1904- )

Article in Observation and Interpretation, ed. S. Körner (Butterworth, London, 1957) 30/54

Royce, Josiah. American philosopher (1855-1916)

Introduction to Poincaré, op. cit. 123/20-21

Russell, Bertrand A. W. British mathematician and philosopher (1872- )

The Analysis of Matter (Dover, New York, 1954) 25/142

Preface to Nicod, op. cit. $126 / 5$

Sainte-Claire Deville, Henri E. French chemist (1818-1881)

125/quoted by Duhem, op. cit., p. 125

Sambursky, Samuel. German-Israeli physicist and historian of science (1900- )

The Physical World of the Greeks, tr. M. Dagut (Routledge \& Kegan Paul, London; Macmillan, New York, 1956) 25/231, 66/174-175, $98 / 40,152 / 233,174 / 7-8,183 / 23-24,189 / 96,287 / 97$

Santayana. George. Spanish-American philosopher (1863-1952)

The Life of Reason (Scribner, New York, 1954) 6/14

Schrödinger, Erwin. Anglo-Austrian physicist (1887-1961)

(I) Science and the Human Temperament, tr. J. Murphy \& W. H. Johnston (Norton, New York, 1935)

(II) What Is Life and other Scientific Essays (Cambridge Univ. Press, 1956)

36/II 207-209, 185/II 159, 232/I 189, 247/II 155, 362/II 196, 368/II $191 \& 196$

Seneca, Lucius A. Roman philosopher (fl. 40 A.D.)

71 quoted by S. Sambursky, op. cit., p. 220

Sherrington, Charles S. British physiologist (1861-1952)

Man on his Nature (Cambridge Univ. Press, 1940) 180/164-166, 379/168-169, 383/400-401

Snow, Charles Percy. British scientist, novelist, and statesman (1905- ) The Two Cultures and the Scientific Revolution (Cambridge Univ. Press, 1959) 103/14, 302/10-11 
Stallo, John Bernhard. German-American jurist and philosopher (18231900)

The Concepts and Theories of Modern Physics, ed. P. W. Bridgman (Harvard Univ. Press, 1960) 98/10-11

TAton, René. Contemporary French historian of science

Reason and Chance in Scientific Discovery, tr. A. Pomerans (Philosophical Library, New York, 1957) 269/147, 277/147

Toulmin, Stephen E. British philosopher (1922- )

The Philosophy of Science (Hutchinson, London; Harper, New York, 1960) 62/92-93, 209/80-82, 292/113

Trotter, Wilfred B. L. British surgeon and physiologist (1872-1939)

Collected Papers (Oxford Univ. Press, 1941) 112/172, 306/156

Tyndall, John. British physicist (1820-1893)

349/quoted by W. Beveridge, op. cit., p. 105

Wallace, Alfred Russel. British naturalist (1823-1913)

315/quoted by W. Beveridge, op. cit., p. 139

WeIzsäcker, Carl Friedrich Baron von. German physicist and philosopher (1912- )

The World View of Physics, 4th ed., tr. M. Grene (Routledge \& Kegan Paul, London; Univ. of Chicago Press, 1952) 35/197, 82/196-197, 84/95, 98/131, 195/190, 221/194, 286/101, 286/131, 290/97, 348/12, 366/179, 374/198

WeyL, Hermann. German-American mathematician (1885-1955)

Mind and Nature (Univ. of Pennsylvania Press, 1934) 7/30-31 Philosophy of Mathematics and Natural Science (Princeton Univ. Press, 1949) 221/156, 260/151, 371/146-147, 372/155

Whewell, William. British historian, educator, and philosopher (17941866)

Novum Organum Renovatum (Parker, London, 1858) 224/114$115,270 / 88,307 / 180,340 / 46$

WhiteHeAD, Alfred North. British mathematician and philosopher (18611947)

Essays in Science and Philosophy (Philosophical Library, New York, 1948) $23 / 73$

Whorf, Benjamin Lee. American linguistic theorist (1897-1941)

Language, Thought, and Reality (Wiley, New York, 1956) 14/213, 99/213-214 \& 221, 325/209-210 
Wittgenstein, Ludwig J. J. Anglo-Austrian philosopher (1889-1951)

Preliminary Studies for the "Philosophical Investigations" (Harper, New York, 1958) 23/5

WöLFFLIN, Heinrich. German historian of art (1864-1945)

324/Principles of Art History

Wollaston, William Hyde. British chemist and physicist (1766-1828)

Article in Phil. Trans. 104 (1814) 252/7

Young, John Zachary. British anatomist (1907- )

Doubt and Certainty in Science (Oxford Univ. Press, 1951) 7/62

Zirkle, Conway. American botanist (1895- )

346/quoted by R. Taton, op. cit., p. 129 



\section{Index}

Boldface numbers signify the pages on which quoted material appears.

Adams, J. C., 371

Agassiz, L., 272

Albers, Josef, 354

Alphonso of Castille, 99

Ames, A., 10

Anaxagoras, 183

Anaximenes, 185

Anderson, C. D., 246

Aquinas, 74, 96, 364

Arago, 256, 257, 263

Archimedes, 70-71, 74, 79, 80, $170,220,252,290$

Aristarchus, 44, 45, 73, 274, 275

Aristotle, 67, 71, 73, 74, 77, 152, $190,213,286,291$

Arrhenius, 267, 268, 316

Averroës, 96

Avogadro, 253, 264, 277

Ayer, A. J., 212

Bacon, 48, 72, 75, 76-77, 79-80, $82,83,91,103,152,158$,

$196,267,313,320,356$

Balmer, 165, 309, 314, 315

Barron, F., 350
Bartlett, F., 13, 156, 281-282, 319

Baudelaire, 344

Becquerel, 334

Bell, A. G., 107, 110

Berkeley, 172, 212

Bernal, J. D., 91

Bernard, C., 39-40, 142, 159, 176, 205-206, 223, 323, 325, 329, $335,340,342-343$

Bernoulli, J., 186

Berthollet, 276

Berzelius, 142, 253, 264, 275, 313, 332

Bessel, 37, 220

Beveridge, W. I. B., 311, 330-331, 340, 344-345

Binkley, 106, 107

Black, J., 270, 273-274

Bode, 114, 132-133

Bohm, D. J., 202-203

Bohr, 29-30, 88, 135, 164, 185, $245,246,278,285,288,314$, 356,381

Boltzmann, 244 
Born, 29, 35, 93, 101, 152, 168, $220,245,246,253,361,376$ 377,381

Boyle, 18, 19, 24, 28, 56, 58, 60, $82,114,117,119-123,124$, 130, 133, 137, 152, 187, 193, 197-198, 220, 221, 222, 294, 317,377

Brahe, Tycho, 78, 100, 156, 157, $163,279,313,327,374$

Brain, W. R., 172

Braithwaite, R. B., 45, 235-236, 353

Bridgman, 92, 97, 127, 178, 230, $231,248,360$

Bronowski, 4, 48, 83, 172, 379

Brown, G. S., 85

Brown, R., 237, 377

Bruner, 228, 229, 341

Bruno, 94, 101

Butterfield, H., 78-80, 105, 346

Campbell, N. R., 241

Cannizzaro, 221, 222, 275

Carathéodory, 244

Carnot, S., 240

Caro, H., 109

Carr, H. A., 204

Carroll, L., 227

Cavendish, 283, 284

Chadwick, 339

Charles, 58, 59, 221

Chevalier, M., 106

Churchman, C. W., 364

Clairaut, 227, 277

Clausius, 317

Cleve, 316

Clifford, WV. K., 4

Cohen, M. R., 26, 163, 168, 203$204,205,223,344,370,381$

Colding, 167

Coleridge, 65
Collingwood, 77

Columbus, 329, 330

Comte, 242, 244, 294, 345, 368

Conant, 4, 85, 107, 111, 157, 259, 293, 306, 338

Condorcet, 379

Copernicus, 44, 45, 69, 70, 73, 74, $78,94,95,96,99,100,101$, $102,177,197,211,226,250$, $252,266,267,268,272,275$, $277,280,281,283,285,323$, $326,328,345,346,347,348$, 349,374

Crick, 346

Crombie, A. C., 97

Crookes, 339, 341

Cusanus, 96

Dalton, 58, 94, 95, 129, 134, 165, $175,220,250,252,253,276$, $277,278,313,345,346,349$

Darwin, C., 94, 95, 102, 267, 268, 303, 307, 312, 313, 321, 324, $339,347,352,360$

Davisson, C. J., 246

de Broglie, L. V., 100-101, 128, 202, 246, 348

de Maistre, 158

Democritus, 184, 186, 187, 250

De Morgan, 271

de Santillana, 251

de Saussure, 154

Descartes, 73, 74, 76, 79, 97, 313

Dewar, 160

Dingle, 85, 87, 99, 174, 240-241, $280,314-315,349,379$

Dirac, 230, 231, 232, 237-238, 245, 246, 269, 271, 287, 382

Doppler, 315

Driesch, 192

du Bois-Reymond, E. H., 187

Duhem, 96, 117, 129, 241, 242, 
243, 244, 245, 248, 250, 251, 261, 263, 269, 347, 357, 363, $371,373,375$

Dulong, 140, 141, 165, 262

Dumas, J. B., 317

du Noüy, 101, 159, 190

Eddington, A., 96, 154, 172, 220, $272,292,293,294$

Edelstein, L., 91, 180

Edison, 109, 110

Ehrlich, P., 331

Einstein, 4, 24-25, 28, 46, 47, 102, 103, 117, 131, 133, 169, 173, $178,179-180,189,211,218$, 220, 224, 239, 245, 257, 267, $272,284,285,288,292,312$, 321-322, 327, 328, 345, 350, $360,370,373,375,377$

Elsasser, 246

Empedocles, 67

Epicurus, 73

Euclid, 53, 57, 65, 72, 73, 88, 126 , $127,214,215,216,217,220$, $225,228,229,234,235,252$, $285,374,375,379$

Fabricius, 96

Faraday, 108, 152, 245, 278, 313, 335

Feigl, H., 262

Fermi, 164, 317

Fierz, 271

Fisher, R. A., 166, 329

Fitzgerald, 262

Fleming, 109, 313, 341, 342

Foucault, 257

Fouillée, 5

Fourier, J. B. J., 88, 317

Frank, P., 359, 375

Franklin, 87

Fresnel, 313

Freud, 41, 94, 95, 160, 272, 353
Frisch, O. R., 329

Galen, 158

Galileo, 44, 48, 49, 51, 67, 74, 79, $81,82,94,96,97,101,114$, $125-127,152,169,173,177$, 180, 195, 197, 198, 206, 212, $222,252,275,288,308,313$, $325,327,328,345$

Galvani, 342

Gamow, G., 175, 234

Gauss, 127, 220, 352

Gay-Lussac, 58, 165, 277

Gerhardt, 317

Germer, L. H., 246

Gibbs, J., 270

Goethe, 153, 154

Graham, T., 58

Haggard, H. W., 71

Hahn, O., 310, 329

Hales, S., 163, 312

Hall, A. R., 64, 79, 80, 173, 211

Hamilton, W. R., 248, 352

Harlan, S. C., 38

Harvey, 96, 158

Head, F. B., 45

Hebb, 6, 8-9, 11-12, 26, 144, 247

Heisenberg, 174-175, 220, 245, 289, 290, 368, 373

Helmholtz, 317, 352

Heraclitus, 36, 183, 187

Hero, 74

Herschel, J. F. W., 45, 69

Hertz, H., 108, 109, 281, 367-368, 369

Hesse, M. B., 231-232, 238, 244, 248

Hevesy, 96

Hillebrand, W. F., 111, 335, 339

Hippocrates, 77

Holton, G. J., 311

Homer, 357, 376 
Hooke, 235, 308, 342

Hume, 196, 378

Hutten, E. H., 231, 232-233, 235, 239, 243-244, 250-251, 2Ss

Huxley, T. H., 4, 95, 313

Huygens, 308

Ingen-Housz, 163, 349

James, Wm., 3, 4, 17, 94, 204, 206, 207-20S, 2SS, 306, 366, 369 Jeans, 93

Jeffreys, H., 1S, 181, 227

Jenner, 345

Johnson, S., 358

Joule, 135, 167

Kant, 126, 127, 170, 171, 172, 289, $360,366,374,379$

Kekulé, 237, 259, 346, 352, 354

Kelvin, 108, 109, 178-179

Kepler, 70, 78, 82, 91, 97, 100, $102,114,123,124,125,133$, $147,156,157,177,211,221$, $222,226,252,275,277,308$ $309,312,313,327,330,331$

Keynes, J. M., 323

Kinnebrook, D., 37, 39

Kirchhoff, G., 317

Koestler, A., 177, 313, 319, 354355

Koyré, 363

Kroeber, A. L., 95, 276-277

Kropotkin, P. A., 352

Kummer, 381

Landé, 201, 203

Langer, S., 8, 15, 33, 70, 169

Lalande, J. de, 335

Lamarck, 348

Laplace, 95, 220, 235, 277

Lavoisier, 94, 106, 10S, 130, 146, $210,235,266,268,273,276$, $284,312,316,317,331,337$, 346

Laurent, 317
Leibniz, 188, 196, 201, 223, 271

Leonardo da Vinci, 313

Leucippus, 187

Leverrier, 371

Lewis, G. N., 206, 224, 254

Liebig, 313, 327

Locke, 95, 212

Lorenz, K., 13, 354

Luther, 349

Lysenko, 272, 304

Mach, 93, 242, 244, 294, 295, 312, $314,328,357,360,361,362$, $365-366,370,379$

Magendie, 333

Malthus, 347, 353

Malus, 333-334

Marconi, 108

Margenau, 10, 11, 65, 200

Marignac, 262

Maskeleyne, N., 37, 39

Maupertuis, 314

Maxwell, J. C., 43, 60, 87, 94, 95, $101,108,110,245,248,278$, 313,369

Mayer, J. R., 167, 316

Mayow, 274

McGeoch, 347

Meitner, 329

Mendel, G., 42, 114, 135-137, 200, $233,234,237,329,330,346$

Mendeleev, 134, 135, 158, 262, $270,271,282$

Merton, R. K., 304

Metchnikoff, 353

Meyer, L., 134

Meyerson, E., 5, 12, 25, 46, 116, $118,167,187,374,379$

Michelson, A. A., 262

Mill, J. S., 11, 16, 4S, 182, 183,

205,225

Millikan, R., 240

Mitscherlich, 334, 335, 340 
Möbius, 381-382

Moore, G. E., 364

Morley, E. W., 262

Moseley, 114, 131, 133-135, 164

Mossotti, 248

Morse, S. F. B., 107

Mowrer, O. H., 26

Nagel, E., 29S, 299

Needham, J., 102

Newton, 46, 58, 69, 73, 74, 82, 88, $91,94,95,97,100,131,133$, $152,153,160,165,173,174$, $175,176-177,181,183,187$, 188, 19S, 199, 201, 206, 207, 210, 211, 212, 213, 219-220, 223, 226, 234, 235, 236, 237, $240,241,242,252,255,267$, 268, 271, 275, 276, 277, 278, 279, 280, 283, 291, 292, 312, $321,327,345,352,370,371$, 374

Nicod, J., 170, 239

Nicolle, 307, 352, 353

Nietzsche, 203, 204, 2S4, 362

Noddack, I., 165, 317

Noyes, H. P., 246, 364-365

Occam, 172

Ohm, 88

Oparin, 175

Oppenheimer, J. R., 4, 103, 2\$9, 294

Osiander, 329

Painlevé, P., 212

Parmenides, 187, 250, 362

Pascal, B., 82, 108, 179, 249, 251, $259,260,271,312,323$

Pasteur, 75, 90, 109, 142, 153, 258, $275,307,313,327,332,335$, $339,340,341,346$

Pauli, 246

Pavlov, 6, 350

Peirce, B., 219
Peirce, C. S., 201, 219, 325

Perier, 82, 261, 312

Perkin, Wm. H., 109

Petit, 140, 141, 165, 262

Philolaus, 177

Piaget, 13

Picasso, P., 111

Pirie, N. WV., 190

Planck, 93, 167, 196, 199, 200, 201-202, 221, 239, 245, 267, $272,285,317,327,345$

Plato, 76, 101

Poincaré, H., 21, 44, 56, 105, 109, $121,126,129,165,186,210$, $217,224,227,245,279,296$, $314,351,352,353,361,367$, 374,375

Poisson, 248

Polanyi, M., 10, 14, 22, 37, 38, 40, 91, 96, 97-98, 100, 157, 158, $167,227,251,271,272,279$, $280,299,300,301,302,303$, $320,322,326,339,339,349$, $350,356-357$

Popper, K. R., 16, 45, 61, 204, 295, 326

Pouchet, 258

Priestley, J., 103, 111, 163, 266, 267, 271, 282, 316, 331, 334, $335,336,337$

Proust, M., 4

Proust, J. L., 146, 276

Prout, Wm., 132, 262, 331

Ptolemy, 70, 74, 77, 91, 99, 211, $260,266,268,275,279,283$, $324,347,376$

Pythagoras, 48, 78, 177, 327

Quine, 261, 262, 263, 357, 35s, 367,368

Randall, M., 254

Rankine, 240

Rayleigh, 111, 334, 335, 338 
Regnault, 294

Reichenbach, 295-296, 311-312, 328

Rheticus, 226

Rhine, J. B., 167, 272

Richet, C. R., 333

Riemann, 127, 217, 375

Roentgen, 281, 334, 341, 345

Rogers, C. R., 333

Rosenfeld, L., 30

Royce, J., 123

Rumford, 280

Russell, B., 25, 33, 34, 126

Rutherford, 135, 142, 164, 339

Ste.-Claire Deville, 125

Sambursky, 25, 66, 70-71, 98, 152,

$174,183,189,287-288$

Santayana, 5-6

Sapir, Edw., 98

Schrödinger, 36, 185, 232, 245, $247,362-363,368$

Seneca, 71

Shaw, G. B., 38

Sherrington, 27, 91, 180-181, 379380,383

Snell, 209

Snow, C. P., 103, 302

Sommerfeld, 278

Spallanzani, 142, 275

Spinoza, 103

Stallo, J. B., 98

Stas, 108

Strassman, F., 310, 329

Stern, Otto, 369

Swammerdam, 342

Szent-Györgi, 96

Szilard, 96

Taton, R., 269, 277

Teller, Edw., 96

Thales, 166
Thompson, W. R., 4

Thomson, G. P., 246

Thomson, J. J., 109, 118, 206

Thomson, Thomas, 331, 332

Titus, J. D., 114, 132-133

Torricelli, 108, 120, 234, 249, 251, $283,312,325$

Toulmin, S. E., 61-62, 209, 248, 292-293

Townley, R., 120

Trotter, W. B. L., 112, 306

Tyndall, 75, 258, 341, 349

Urey, 271

van der Waals, 59, 117

Vavilov, 304

Vesalius, 96, 158

Von Bekesy, 96

Von Brueck, 181

Von Frisch, 181

Von Karman, 96

von Laue, 251

Von Neumann, 96

Wallace, A. R., 314-315, 347, 353

Waterston, 317

Weizsäcker, 35, 82, 84, 86, 98, $195,221,286,290,348,365$ 366,374

Weyl, H., 7, 221, 260, 371-372

Whewell, Wm., 106, 224, 270, 306-307, 340

Whitehead, A. N., 23

Whorf, B. L., 14, 98, 99, 325

Wigner, E., 96

Williams, R. J., 37

Wittgenstein, 23

Wölfflin, H., 324

Wollaston, Wm., 252-253

Young, J. Z., 7, 313

Zinsser, H., 331

Zirkle, C., 346 







,

9) 